\title{
Anachronismen: Historiografie und Kino
}

Bildteil

\author{
Inaugural-Dissertation \\ zur Erlangung des Grades eines \\ Doktors der Philosophie (Dr. phil.) \\ an der Fakultät Medien \\ der Bauhaus-Universität Weimar \\ vorgelegt von \\ André Wendler (Weimar) \\ 18. Januar 2012
}




\section{Abbildungen Einleitung}

o.1 Teddy/Andrew mit der rückwärts rauchenden Zigarette, SHUTTER ISLAND.

0.2 Begegnung von Marcus Antonius und Kleopatra in Tarsus. Kupferstich aus NeumARK, 1651, Georg Neumarks von Mühlhausen aus Thür: Verhochteutschte Kleopatra, o. S.

0.3 Festmahl des Bacchus. Kupferstich aus NeumarK, 1651, Georg Neumarks von Mühlhausen aus Thür: Verhochteutschte Kleopatra, o. S. 0.4 Seeschlacht von Actium. Kupferstich aus NeumARK, 1651, Georg Neumarks von Mühlhausen aus Thür: Verhochteutschte Kleopatra, o. S. o.5 Tod der Kleopatra. Kupferstich aus Neumark, 1651, Georg Neumarks von Mühlhausen aus Thür: Verhochteutschte Kleopatra, o. S.

\section{Abbildungen Kapitel 1}

1.1 Dr. Cawley an der Tafel, Shutter IsLAND

1.2 KZ-Häftlinge in der Erinnerung Teddys/Andrews, SHUtTeR IsLAND, Einst. 8, Sequenzprotokoll (SP) 1

1.3 Kommandantenbüro in der Erinnerung Teddys/Andrews, SHUTTER IsLAND, Einst. 26, SP 1

1.4 Kommandant von Dachau in der Erinnerung Teddys/Andrews, SHUtTeR IsLAND, Einst. 53, SP 1

1.5 Plattenspieler im Salon Dr. Cawleys, Shutter IsLAND, Einst. 6, SP 1 1.6 Plattenspieler in Dachau, Shutter Island, Einst. 39, SP 1

1.7 Plattenspieler in Dachau, Shutter Island, Einst. 40, SP 1

1.8 Plattenspieler in Dachau, Shutter Island, Einst. 41, SP 1

1.9 Plattenspieler in Dachau, Shutter IsLAND, Einst. 42, SP 1

1.10 Totale des Salons Dr. Cawleys, ShUtTer IsLAND, Einst. 14, SP 1

1.11 Dr. Naehring im Salon Dr. Cawleys, ShutTER IsLAND, Einst. 11, SP 1

1.12 Vogelperspektive in den Erinnerungen Teddys/Andrews, SHUTTER Island, Einst. 43, SP 1

1.13 Froschperspektive in den Erinnerungen Teddys/Andrews, SHUtTeR IsLAND, Einst. 45, SP 1

1.14 Tor des KZ Dachau im Film, ShUtTer IsLAND

1.15 Tor des KZ Auschwitz, Yad Vashem Photo Archive (5353/250), Stanisław Mucha, http://collections.yadvashem.org/ photosarchive/en-us/64935.html (Zugriff: 14.12.2011).

1.16 Tor des KZ Dachau 2005, Yad Vashem Photo Archive (8603/5), Daniel Brunod, http://collections.yadvashem.org/photosarchive/ en-us/7323641.html (Zugriff: 14.12.2011).

1.17 "Young and old survivors in Dachau cheer approaching U.S. troops. Among those pictured are Juda Kukiela (middle), Tevya 
Grojs (second from the right), David Moszkowicz (fourth from the left), Szmulek Rozental (third from the left) and Gyorgy Laszlo Spiegel (second from the left).«, 29.04.1945, United States Holocaust Memorial Museum (\#45075), http://resources. ushmm.org/inquery/uia_doc.php/photos/11497?hr=null (Zugriff: 14.12.2011).

1.18 Palas der Wartburg, Eisenach am 24.05.2010, André Wendler

1.19 Sängerlaube im Sängersaal der Wartburg, Eisenach am 06.02.2010, André Wendler

1.20 Moritz von Schwind, Sängerkriegsfresko im Sängersaal der Wartburg, Eisenach (1855) am 24.05.2010, André Wendler

1.21 Foto des Sängersaals der Wartburg aus der Sängelaube mit dem Fresko von Schwinds ca. 1910, Foto der Königlich-Preußischen Messbild Anstalt.

1.22 VON SCHWIND, SÄNGERKRIEgSFRESKo, DETAIL.

1.23 Detailfotografie des Sängersaals am 24.05.2010, André Wendler

1.24 Detail aus von RitGEN, 1859/1876, Der Führer aufder Wartburg, S. 115.

1.25 Fra Angelico, Sacra Conversazione, genannt Die Madonna der Schatten. Zwischen 1438 und 1450. Fresko und Tempera. Kloster San Marco, Ostkorridor, Florenz. nach Didi-HubeRMAN, 1990/1995, Fra Angelico, S. 32, Abb. 3.

\section{Abbildungen Kapitel 2}

2.1 Anfang von Einst. 86, ChroniK Der AnNA MagdalenA BACH

2.2 Ende von Einst. 86, ChroniK der Anna Magdalena BaCH

2.3 Bach-Dokumente I, Buchseite mit einem Faksimile nach S. 176

2.4 Bach-Dokumente I, S. 34

2.5 Anfang von Einst. 40, Chronik der Anna Magdalena Bach

2.6 Anfang von Einst. 97, CHRONiK Der Anna MagdalenA BACH

2.7 Ende von Einst. 97, Chronik der Anna Magdalena Bach

2.8 Einst. 17, Chronik der AnNa Magdalena BaCH

2.9 Einst. 22, Chronik der Anna Magdalena Bach

2.10 Einst. 28, ChroniK Der AnNa Magdalena BACH

2.11 Einst. 30, Chronik der AnNa Magdalena BaCH

2.12 Zirkulierende Referenz nach Bruno LATOUR, 1999/200o, Die

Hoffnung der Pandora. Frankfurt am Main, S. 85, Abb. 2.21

2.13 Einst. 41, Chronik Der AnNa Magdalena BaCH

2.14 Anfang von Einst. 37, Chronik der Anna Magdalena BaCH

2.15 Ende von Einst. 37, Chronik der AnNa Magdalena BaCH

2.16 Schichtungen kinematografischer Materialien in Einstellungen 90-98, Chronik der Anna Magdalena BaCH 


\section{Abbildungen Kapitel 3}

3.1 Einst. 1 nach Sequenzprotokoll 3, Cleopatra

3.2 Einst. 2 nach SP 3, Cleopatra

3.3 Einst. 38 nach SP 3, Cleopatra

3.4 Einst. 39 nach SP 3, CLEOPATRA

3.5 Einst. 40 nach SP 3, Cleopatra

3.6 Einst. 53 nach SP 3, CLEOPATRA

3.7 Einst. 60 nach SP 3, Cleopatra

3.8 20Th Century-Fox Corporation (Hg.), 1963, Cleopatra. New York, S. [5].

3.9 Mechanische Uhr im Hause Mark Antonys. Cleopatra.

3.10 Bett auf Cleopatras goldener Barke. CLEOPATRA

3.11 Schatten vom Filmkulissengerüst auf Cleopatras Sphinx.

Cleopatra

3.12 Zeitgenössischer Trailer zu CLEOPATRA

3.13 Zeitgenössischer Trailer zu CLEOPATRA

3.14 Fotografisches Bild, CleOPATRA

3.15 Gemaltes Bild, Cleopatra

3.16 Beschädigtes Fresko, Cleopatra

3.17 Schiffsmodelle in Cleopatras Palast in Alexandria, Cleopatra

3.18 Cleopatra am Modell der Bucht von Actium, Cleopatra

3.19 Modell der Schlacht von Actium auf Cleopatras Schiff,

Cleopatra

3.20 Modell der Schlacht von Actium auf Cleopatras Schiff,

Cleopatra

3.21 Die Bucht von Actium mit echten Schiffen und Matte Paintings,

Cleopatra

3.22 Die fünf realen Schiffe des Drehs, CLEOPATRA

3.23 POV auf die Kohlenschale, Cleopatra

3.24 POV mit Bildern vom Forum, Cleopatra

3.25 POV mit Bildern vom Forum und Cleopatra, Cleopatra

3.26 Cleopatra in ihrem Bad, Cleopatra

3.27 Cleoaptra erprobt Make-up-Varianten, Cleopatra

3.28 Cleopatra bei den Bauarbeiten an ihrem Mausoleum,

Cleopatra

3.29 Cleopatra im Streit mit Mark Antony, Cleopatra

3.3o Cleopatra im Streit mit Mark Antony, CleOPATRA

3.31 »Cleopatra-Siegel« der 2oth Century-Fox, VOGUE, Vol. 142, Nr.

3, (1. Aug. 1963)

3.32 Anzeige für Oliver Gintel, VOGUE, Vol. 142, Nr. 3

3.33 Anzeige für Whiting \& Davis, VOGUE, Vol. 142, Nr. 3

3.34 Anzeige für Dan Millstein Inc, VOGUE, Vol. 142, Nr. 3 
3.35 Anzeige für Roamer-Medana Watch Corporation, VOGUE, Vol.

142, Nr. 3

3.36 Anzeige für Tuvaché, VOGUE, Vol. 142, Nr. 3

3.37 Anzeige für JR.Sophisticates, VOGUE, Vol. 142, Nr. 3

3.38 Anzeige für Merriwig International, VOGUE, Vol. 142, Nr. 3

3.39 Anzeige für Jacqueline Cochran, VOGUE, Vol. 142, Nr. 3

3.40 Anzeige für Revlon Sphinx Eye Makeup, 196oer Jahre

3.41 Gebrauchsanleitung des Revlon Sphinx Eye Makeup

3.42 Anzeige für Bates Fabrics, VOGUE, Vol. 142, Nr. 3

3.43 Domenico Beccafumi: Kleopatra. 1508-1510, Öl auf Holz, 75,5 ×

$47 \mathrm{~cm}$, Musée Bonnat, Bayonne

3.44 Alessandro Turchi: Der Tod der Kleopatra. 1640, Öl auf Leinwand, $255 \times 267 \mathrm{~cm}$, Louvre, Paris.

3.45 Giambattista Tiepolo: Das Treffen von Antonius und Kleopatra. ca. 1746, Öl auf Leinwand, $66 \times 38 \mathrm{~cm}$, National Gallery of Scotland, Edinburgh

3.46 Jan de Bray: Das Bankett von Antonius und Kleopatra. 1669, Öl auf Leinwand, 248,9 × 190,5 cm, The Currier Gallery of Art, Manchester, New Hampshire

3.47 Anton Raphael Mengs: Augustus und Kleopatra. 176o, Öl auf Leinwand, $306 \times 212 \mathrm{~cm}$, Stourhead House, Wiltshire

3.48 Louis Gauffier: L'Entrevue d'Auguste et de Cléopâtre après la Bataille d'Actium. 1788, Öl auf Leinwand, 83,8 × 112,5 cm, National Gallery of Scotland, Edinburgh

3.49 Hist. Foto der Wandbemalung im Neuen Museum Berlin. Messling, 1997, Die ägyptische Abteilung im Neuen Museum zu Berlin, Abb. 9, S. 83

3.5o Hist. Foto der Wandbemalung im Neuen Museum mit Bezifferung neben den Kartuschen. Messling, 1997, Die ägyptische Abteilung im Neuen Museum zu Berlin, Abb. 17, S. 97 3.51 Frederick Arthur Bridgman: Kleopatra auf den Terrassen von Philae. 1896, Öl auf Leinwand, 75,9 × 117,2 cm, Dahesh Museum of Art, New York

3.52 Georges Antoine Rochegrosse: Altes Ägypten oder Kleopatra und ihr Gefolge. ca. 189o, Öl auf Leinwand, $65 \times 82 \mathrm{~cm}$, Privatsammlung

3.53 Alexandre Cabanel: Kleopatra erprobt die Wirkung des Giftes an zum Tode Verurteilten. 1887, Öl auf Leinwand, $165 \times 290 \mathrm{~cm}$, Koninklijk Museum voor schone Kunsten, Anvers

3.54 Henri Blaise François Dejussieu: Kleopatra und der Sklave. 1863, Öl auf Leinwand, $82 \times 66 \mathrm{~cm}$, Musée Denon, Chalon-sûr-Saône 3.55 Jean André Rixens: Der Tod der Kleopatra. 1874, Öl auf Leinwand, $200 \times 290$ cm, Musée des Augustins, Toulouse 
3.56 John Collier: Der Tod der Kleopatra. 189o, Öl auf Leinwand, 395

$\times 315 \mathrm{~cm}$, Gallery Oldham, Oldham

3.57 Elizabeth Taylor als Cleopatra in VOGUE, Vol. 139, Nr. 2, S. 71

3.58 Cleopatras ınatürlicher Look`, CLEOPATRA

\section{Abbildungen Kapitel 4}

4.1 Einst. aus der Titelsequenz von CARAVAgGIO

4.2 Einst. aus der Titelsequenz von CARAVAGgio

4.3 Einst. aus der Titelsequenz von CARAVAgGio

4.4 Einst. 1 nach Sequenzprotokoll 4, CARAVAgGio

4.5 Einst. 2 nach SP 4, CARAVAGgIO

4.6 Einst. 3 nach SP 4, CARAVAGgIO

4.7 Einst. 4 nach SP 4, Caravaggio

4.8 Caravaggio: Reuige Magdalena. 1594, 122,5 × 98,5 cm, Galleria

Doria Pamphilj, Rom.

4.9 Einst. 30 nach SP 4, Caravaggio

4.10 Johannes Vermeer: Allegorie der Malerei. ca. 1666-68, Öl auf

Leinwand, $120 \times 100 \mathrm{~cm}$, Kunsthistorisches Museum, Wien.

4.11 Vincenzo Giustiniani mit einem Taschenrechner. Einst. aus

Caravaggio, Sequ. 27

4.12 Giustiniani und Kardinal del Monte beim Essen. Einst aus

Caravaggio, Sequ. 27

4.13 Ranuccio repariert ein Motorrad. Einst. aus CARAVAggio, Sequ. 29

4.14 Lena am Fenster. Einst. aus CARAVAggio, Sequ. 29

4.15 Baglione an der Schreibmaschine. Einst. aus CARAVAGgio, Sequ. 31

4.16 Baglione mit FMR. Einst. aus CARAVaggio, Sequ. 31

4.17 Caravaggio: Berufung des hl. Matthäus. 1600, $322 \times 340$ cm, San

Luigi dei Francesi, Cappella Contarelli, Rom.

4.18 Baglione in der Wanne. Einst. aus CARAvaggio, Sequ. 35

4.19 Jacques-Louis David: Der Tod des Marat. 1793, Öl auf Leinwand, $162 \mathrm{~cm} \times 128 \mathrm{~cm}$, Königliche Museen der Schönen Künste, Brüssel.

4.20 Jerusaleme mit dem Medusenschild. Einst. aus CARAVAGgio,

Sequ. 3

4.21 Amor als Sieger im Film. Einst. aus Caravaggio, Sequ. 28

4.22 Detail aus Caravaggio: Amor als Sieger. 1601/o2, $156 \times 113$ cm, Staatliche Museen, Gemäldegalerie, Berlin.

4.23 Michele malt den Fruchtschälenden Knaben. Einst. aus

Caravaggio, Sequ. 5

4.24 Michele malt die Ermordung des hl. Matthäus. Einst. aus

Caravaggio, Sequ. 17

4.25 Kardinal del Monte und Michele am halb fertigen Bacchino malato. Einst. aus CARAvaggio, Sequ. 7 
4.26 Michele wie der Jüngling, von einer Eidechse gebissen. Einst. aus CARAVAggio, Sequ. 9

4.27 Caravaggio: Jüngling, von einer Eidechse gebissen. $66 \times 49,5 \mathrm{~cm}$, National Gallery, London.

4.28 Modelle für die erste Version des Martyrium des hl. Matthäus.

Einst. aus CARAVaggio, Sequ. 13

4.29 Modelle für die erste Version des Martyrium des hl. Matthäus.

Einst. aus CARAVAggio, Sequ. 13

4.30 Modelle für die erste Version des Martyrium des hl. Matthäus.

Einst. aus CARAVAggio, Sequ. 13

4.31 Michele und Pasqualone. Einst. aus CARAVAggio, Sequ. 57

4.32 Tableau vivant der Grablegung Christi. Einst. aus CARAVAGgio,

Sequ. 57

4.33 Derek JARMAN, 1986, Derek Jarman's Caravaggio. London,

S. 110-111.

4.34 Michele als Bacchus. Einst. aus CARAvaggio, Sequ. 6

4.35 Michele als Knabe mit dem Früchtekorb. Einst. aus CARAVAgGIO,

Sequ. 6

4.36 Caravaggio: Knabe mit Früchtekorb. 1593/94, 70 × 67 cm, Museo

e Galleria Borghese, Rom.

4.37 Michele, Lena und Ranuccio. Einst. aus Caravaggio, Sequ. 30 4.38 Pipo und Jerusaleme. Einst. aus CARAVAggio, Sequ. 30

Die Abbildungen aus den Filmen sind folgenden DVDs entnommen:

CARAVAggio: Salzgeber, D181, (2009).

Chronik der Anna Magdalena Bach, New Yorker Films Video, NYD 01805, (2005)

Cleopatra: 2oth Century-Fox Home Entertainment, 01143, (2002).

SHUTter IslAND: Concorde Home Entertainment, 2782, (2010).

Alle Reproduktionen von Gemälden sind gemeinfreie Abbildungen. 


\section{Abbildungen Einleitung}

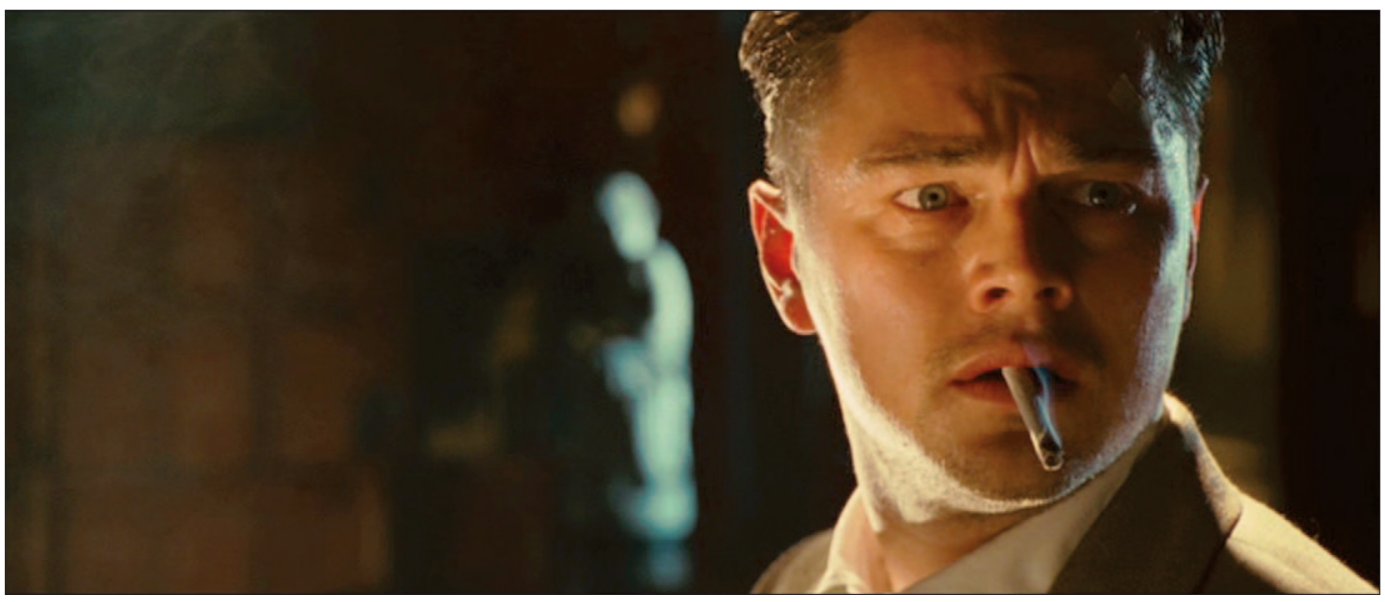

Abb. 0.1 - Teddy/Andrew mit der rückwärts rauchenden Zigarette, SHUTTER IsLAND.

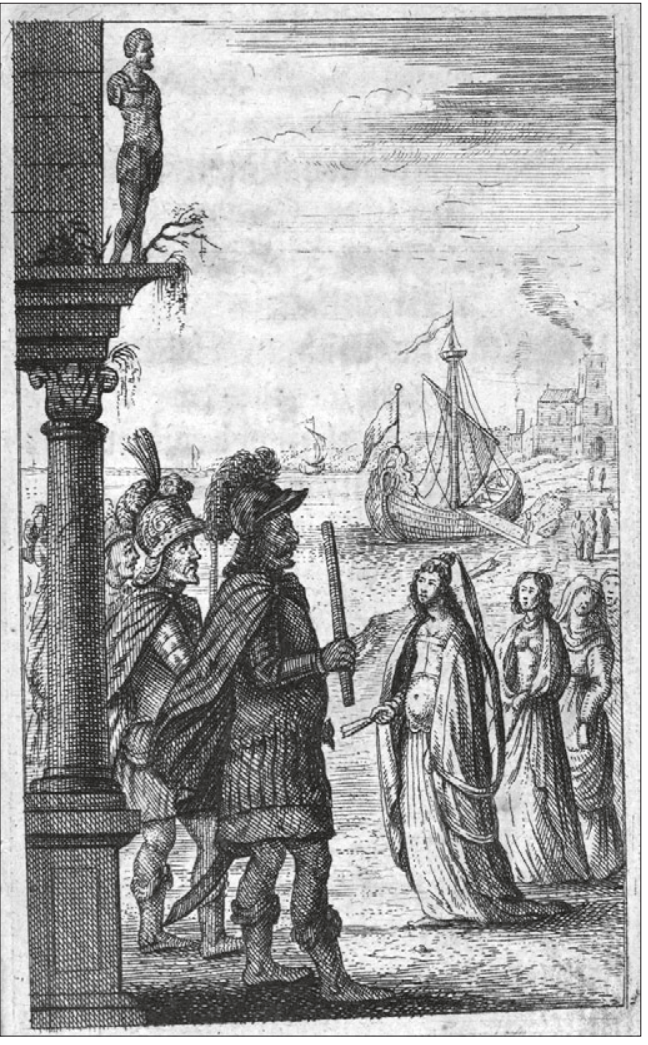

Abb. 0.2 - Begegnung von Marcus Antonius und Kleopatra in Tarsus. Kupferstich aus NEUMARK, 1651, Georg Neumarks von Mühlhausen aus Thür: Verhochteutschte Kleopatra, o. S. (Originalgröße).

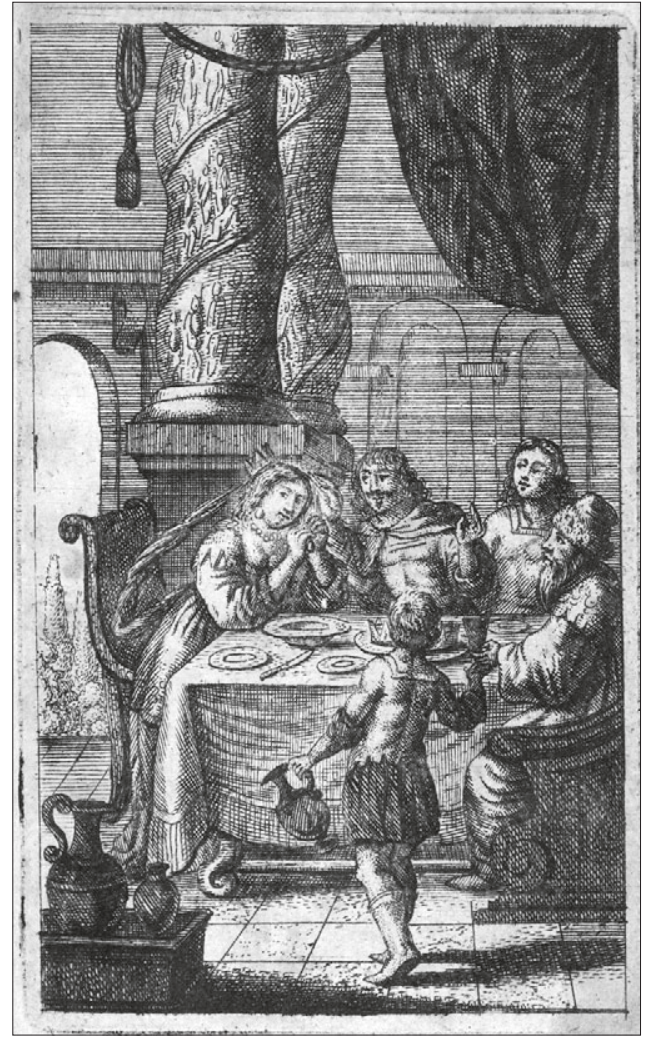

Abb. o.3 - Festmahl des Bacchus. Kupferstich aus NeUMARK, 1651, Georg Neumarks von Mühlhausen aus Thür: Verhochteutschte Kleopatra, o. S. (Originalgröße). 


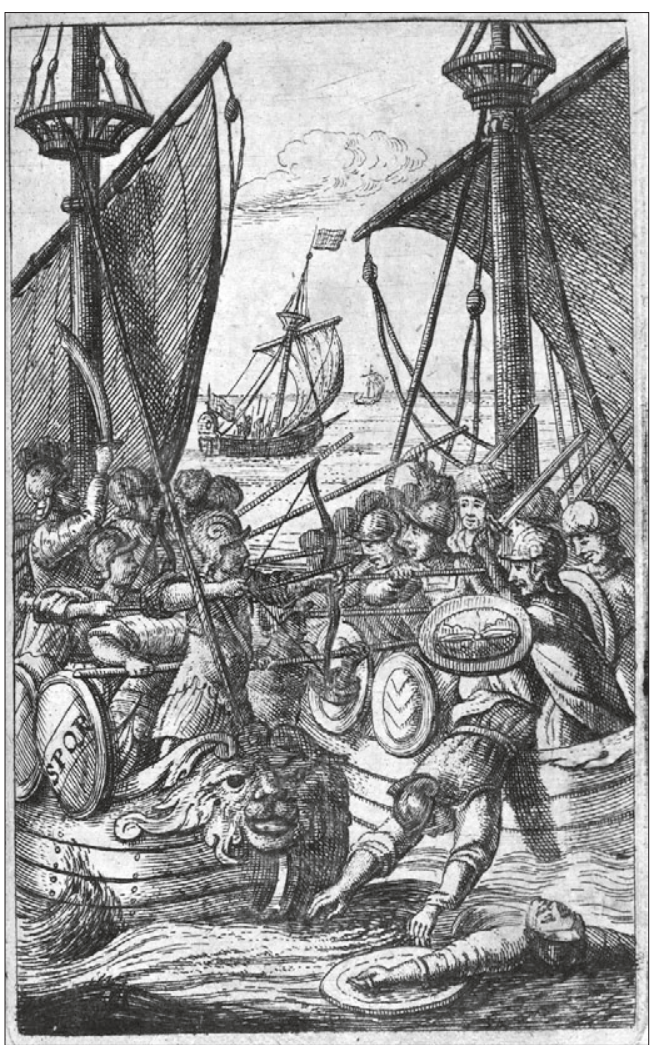

Abb. o.4 - Seeschlacht von Actium. Kupferstich aus NeUmark, 1651, Georg Neumarks von Mühlhausen aus Thür: Verhochteutschte Kleopatra, o. S. (Originalgröße).

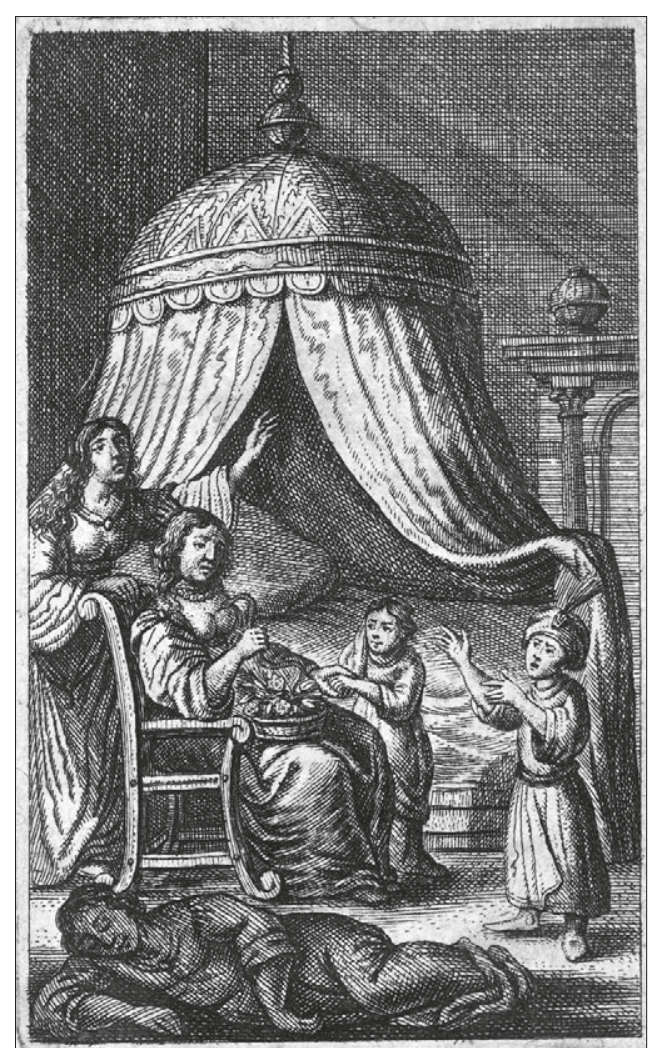

Abb. 0.5 - Tod der Kleopatra. Kupferstich aus NeUMARK, 1651, Georg Neumarks von Mühlhausen aus Thür: Verhochteutschte Kleopatra, o. S. (Originalgröße). 


\section{Abbildungen Kapitel 1}

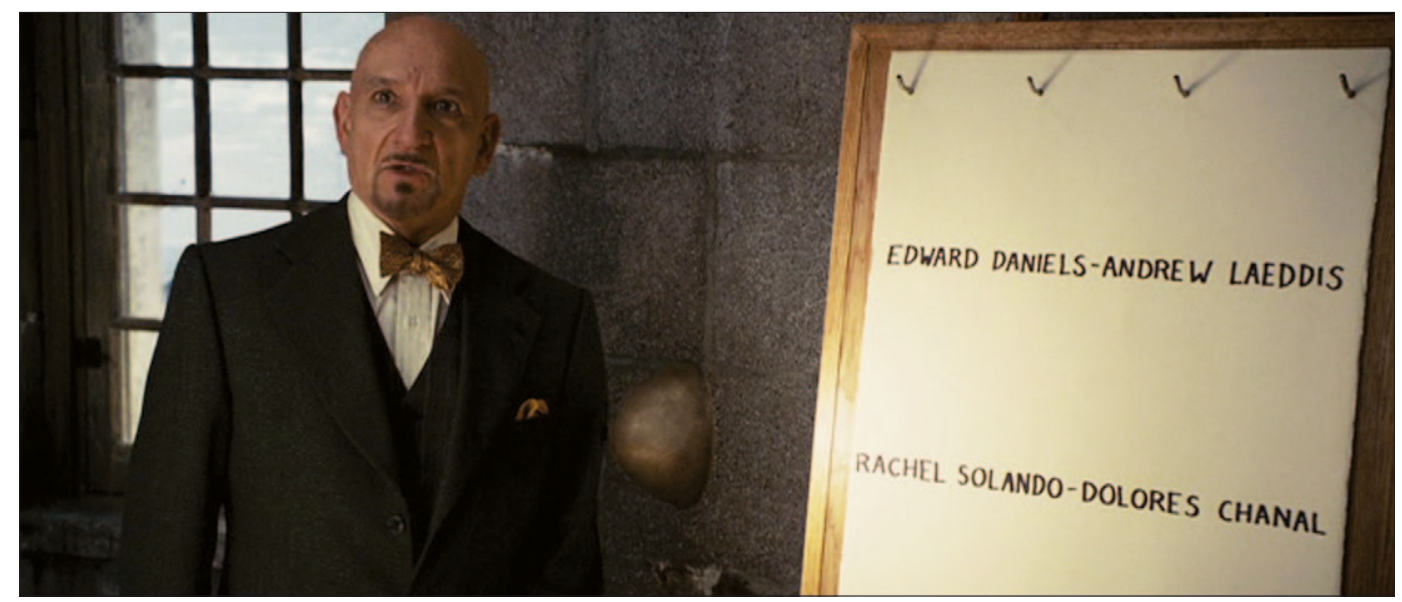

Abb. 1.1 - Dr. Cawley an der Tafel, Shutter IsLAND

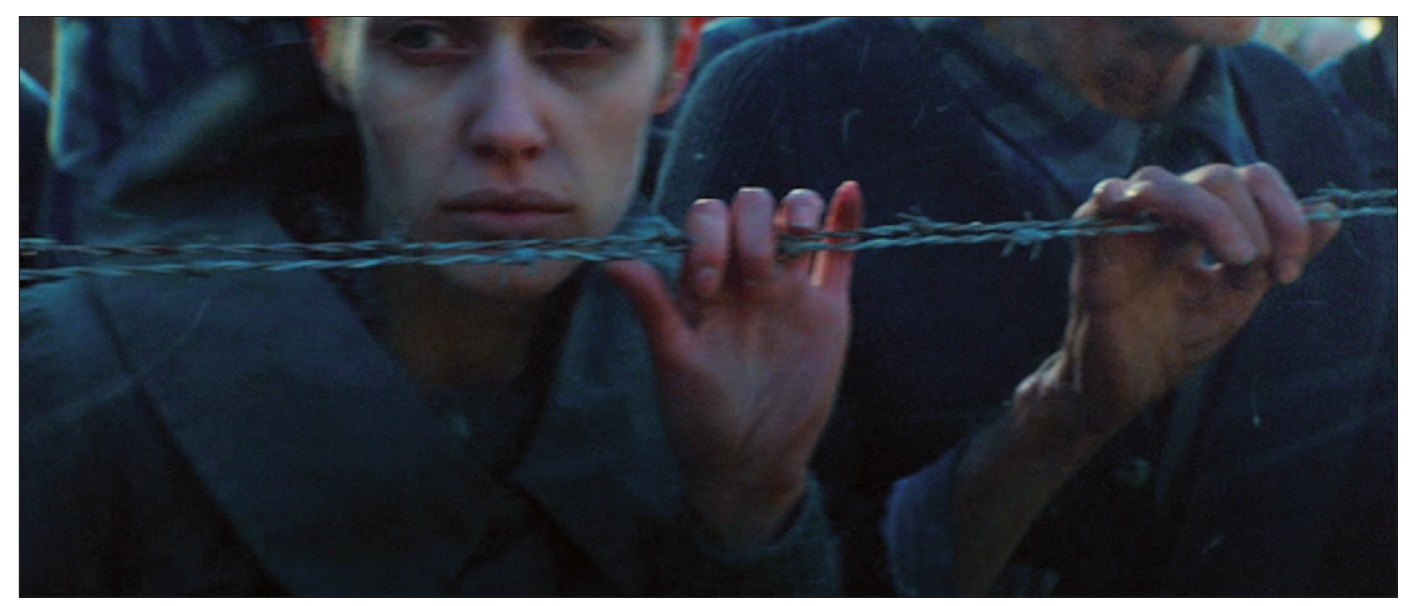

Abb. 1.2 - KZ-Häftlinge in der Erinnerung Teddys/Andrews, SHUTter IsLAND, Einst. 8, Sequenzprotokoll (SP) 1

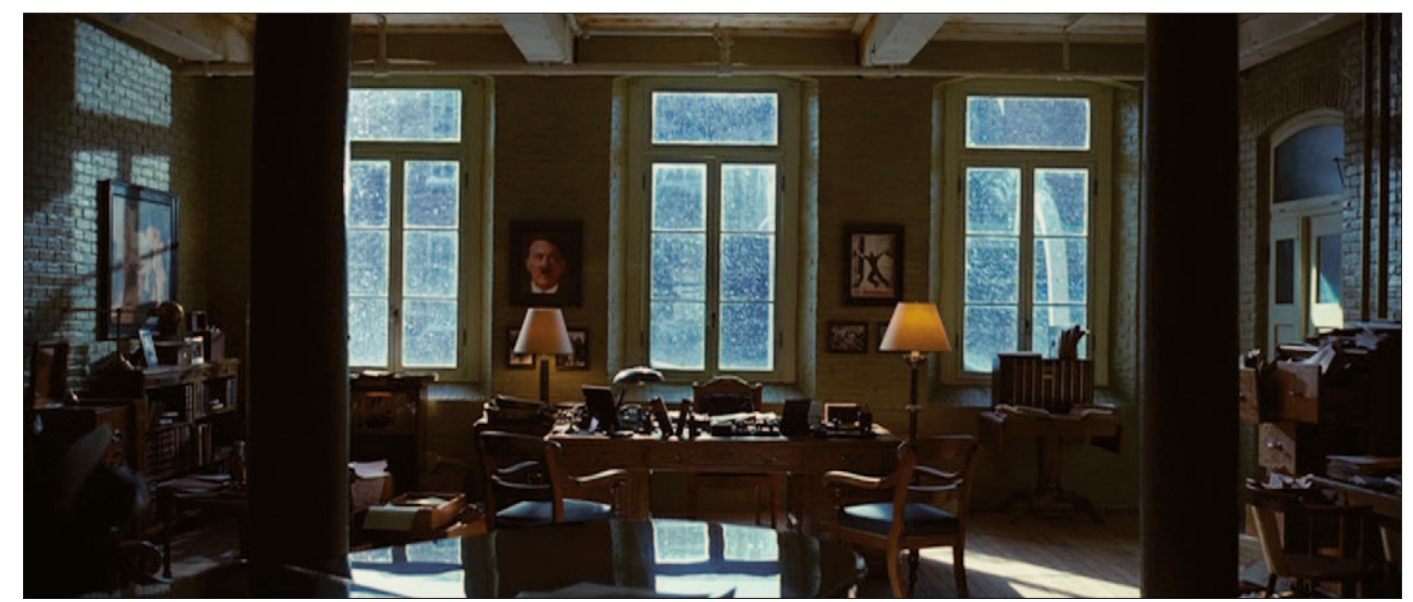

Abb. 1.3 - Kommandantenbüro in der Erinnerung Teddys/Andrews, SHUTTER IsLAND, Einst. 26, SP 1 


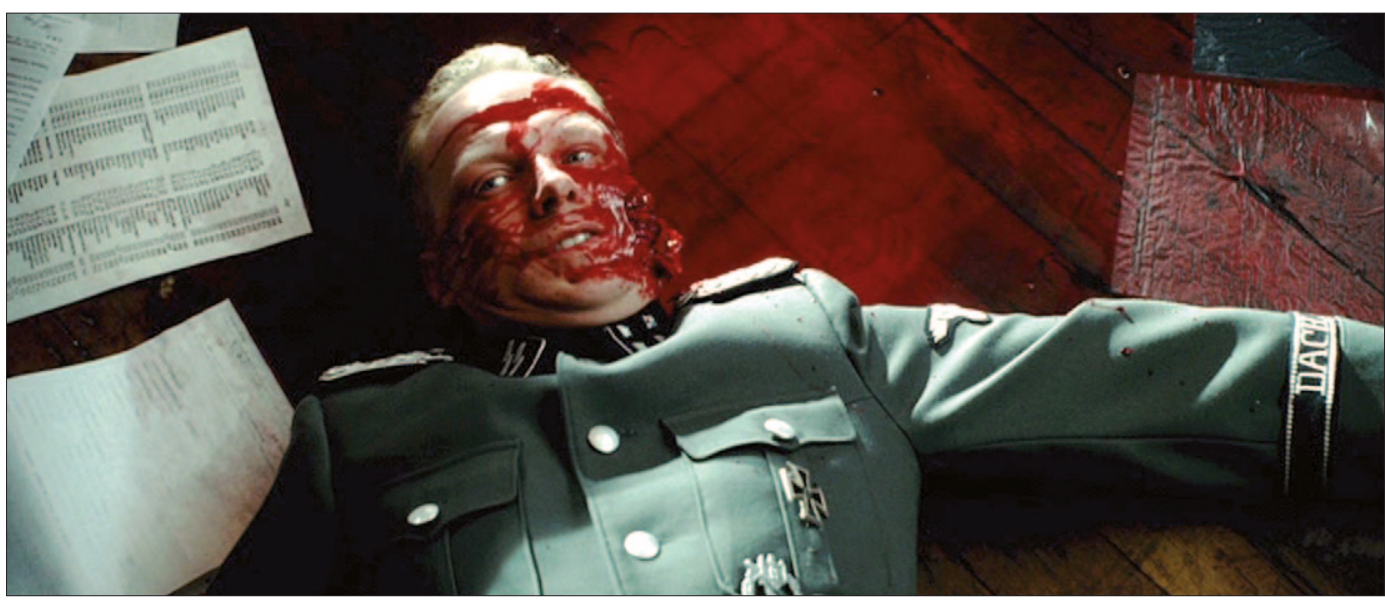

Abb. 1.4 - Kommandant von Dachau in der Erinnerung Teddys/Andrews, SHUTteR IsLAND, Einst. 53, SP 1

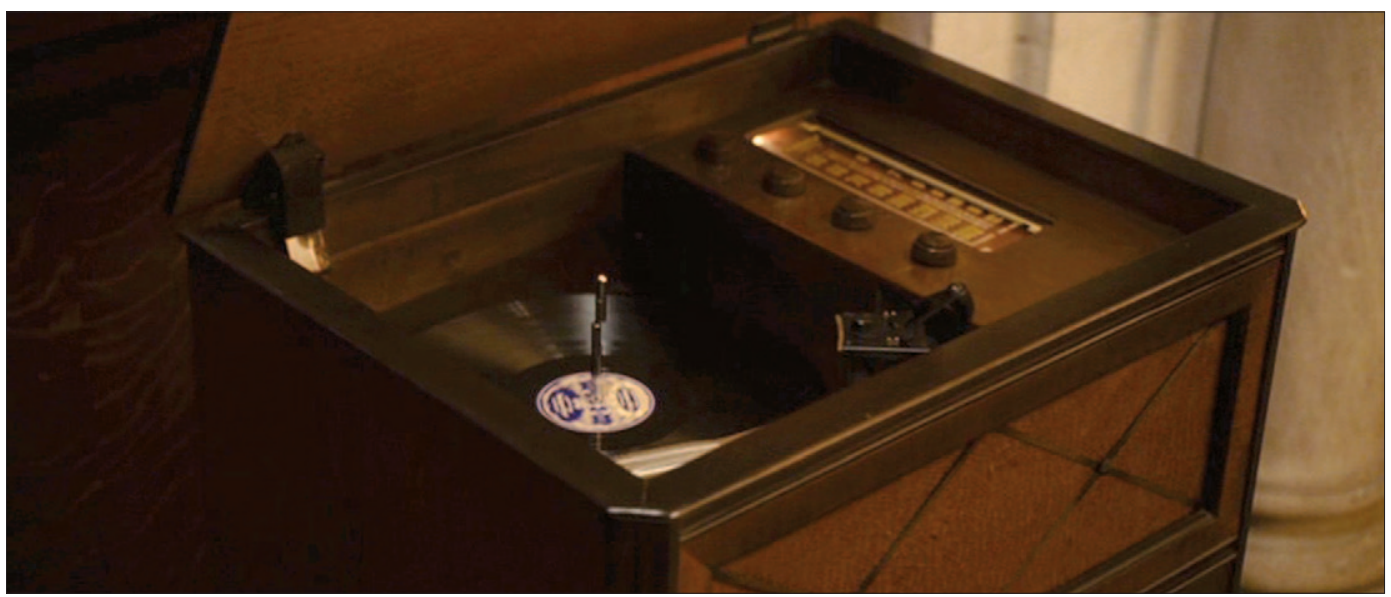

Abb. 1.5 - Plattenspieler im Salon Dr. Cawleys, Shutter Island, Einst. 6, SP 1

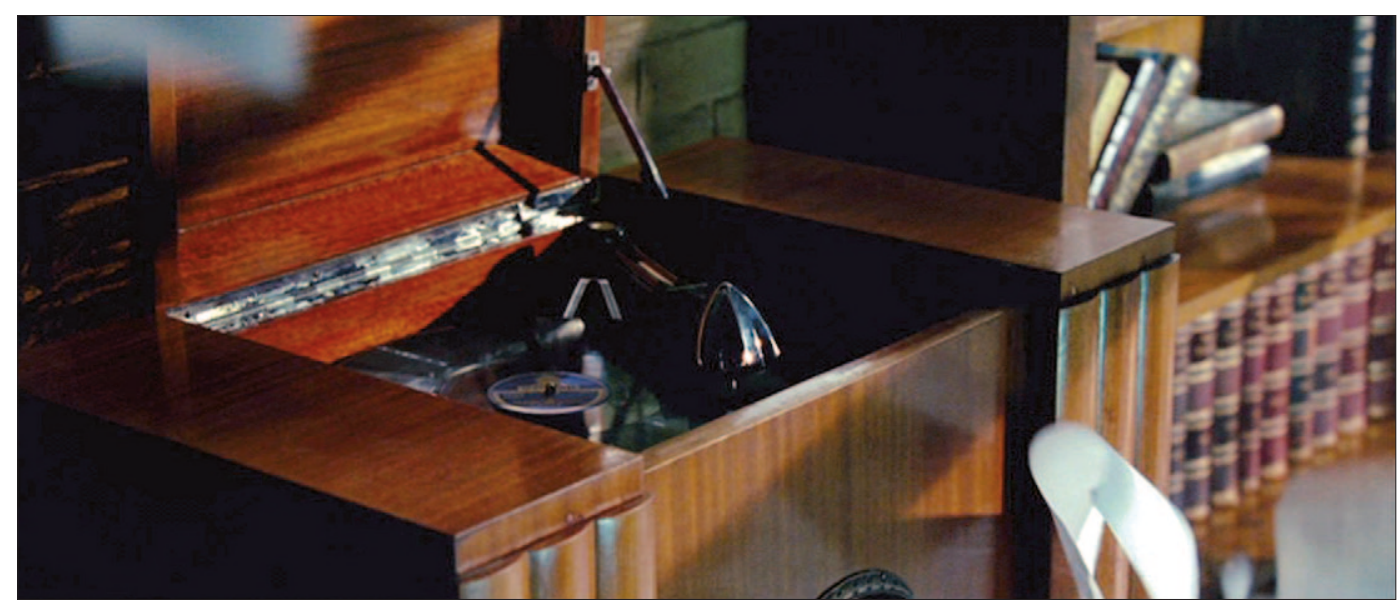

Abb. 1.6 - Plattenspieler in Dachau, SHUtTER IsLAND, Einst. 39, SP 1 


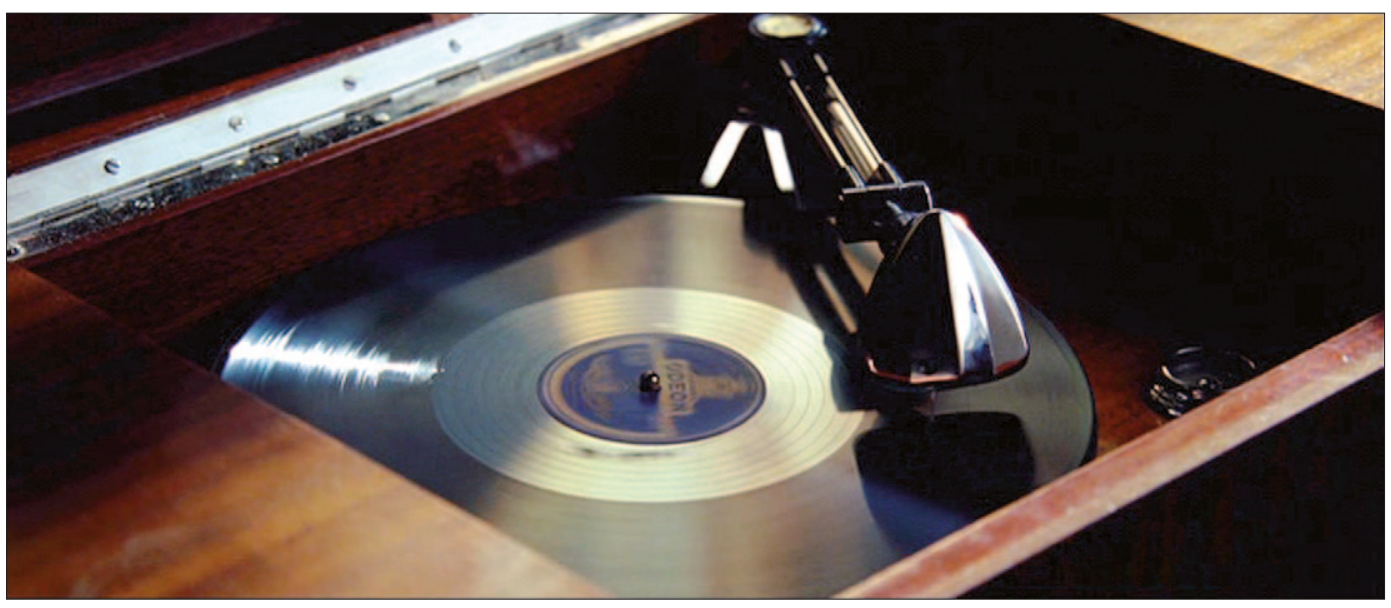

Abb. 1.7 - Plattenspieler in Dachau, SHUtTER IsLAND, Einst. 4O, SP 1

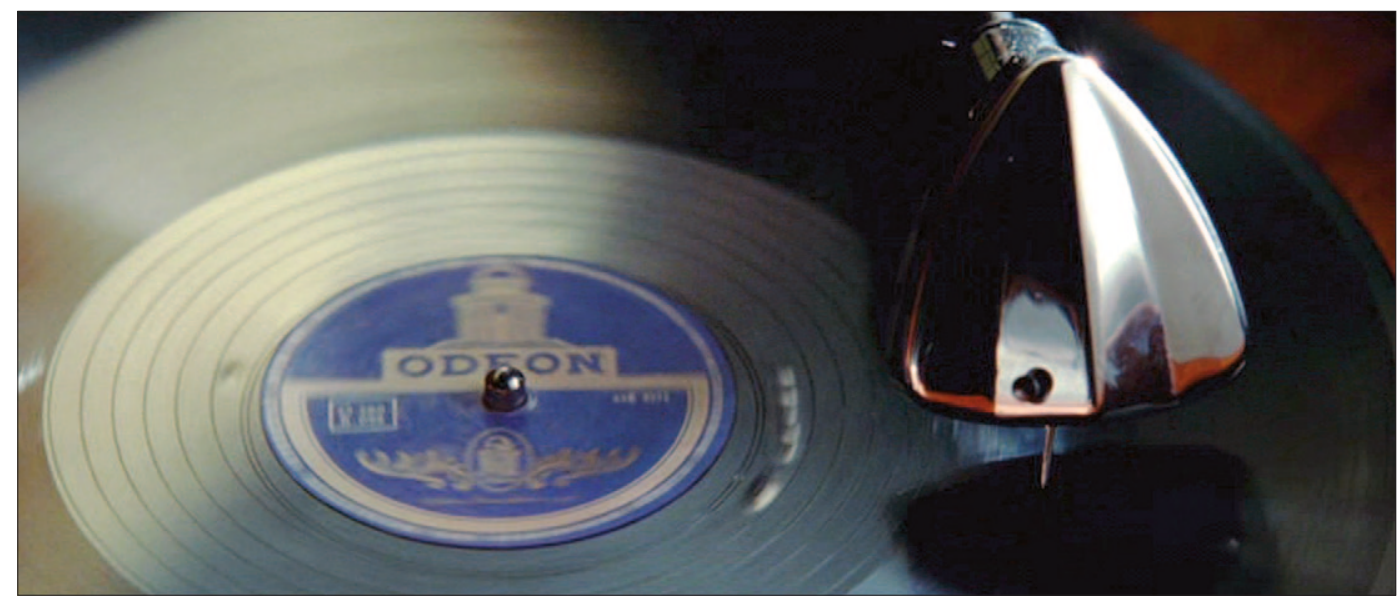

Abb. 1.8 - Plattenspieler in Dachau, SHUtter Island, Einst. 41, SP 1

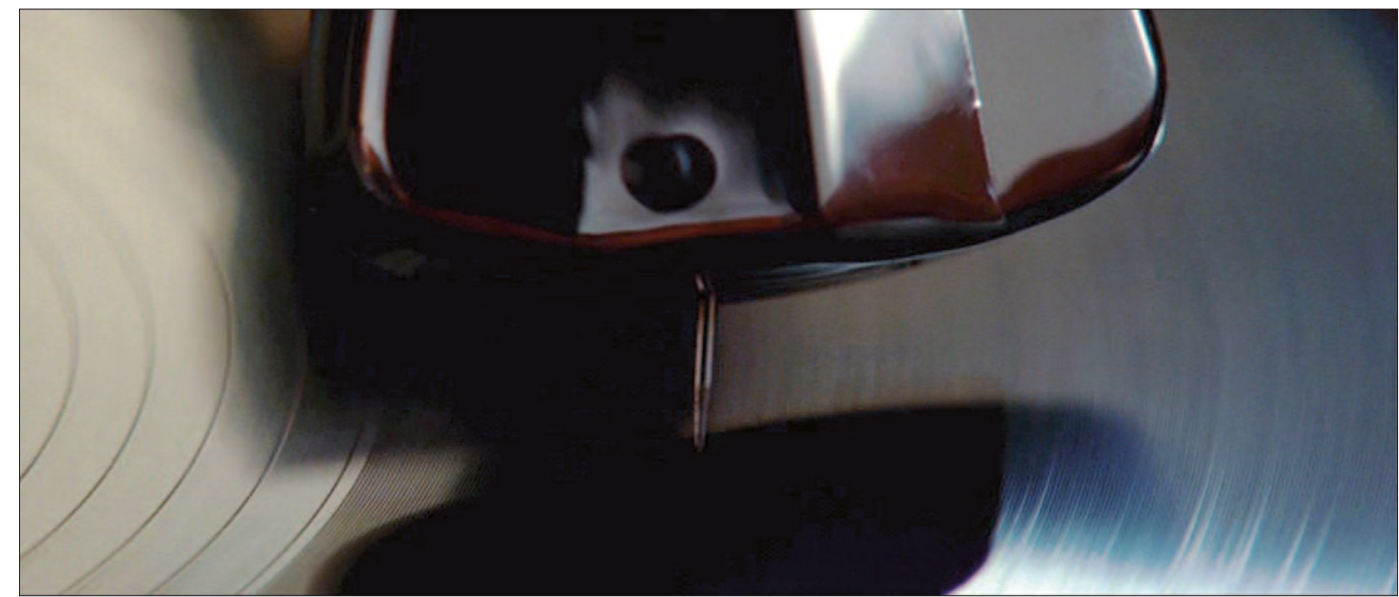

Abb. 1.9 - Plattenspieler in Dachau, SHUTTER IsLAND, Einst. 42, SP 1 


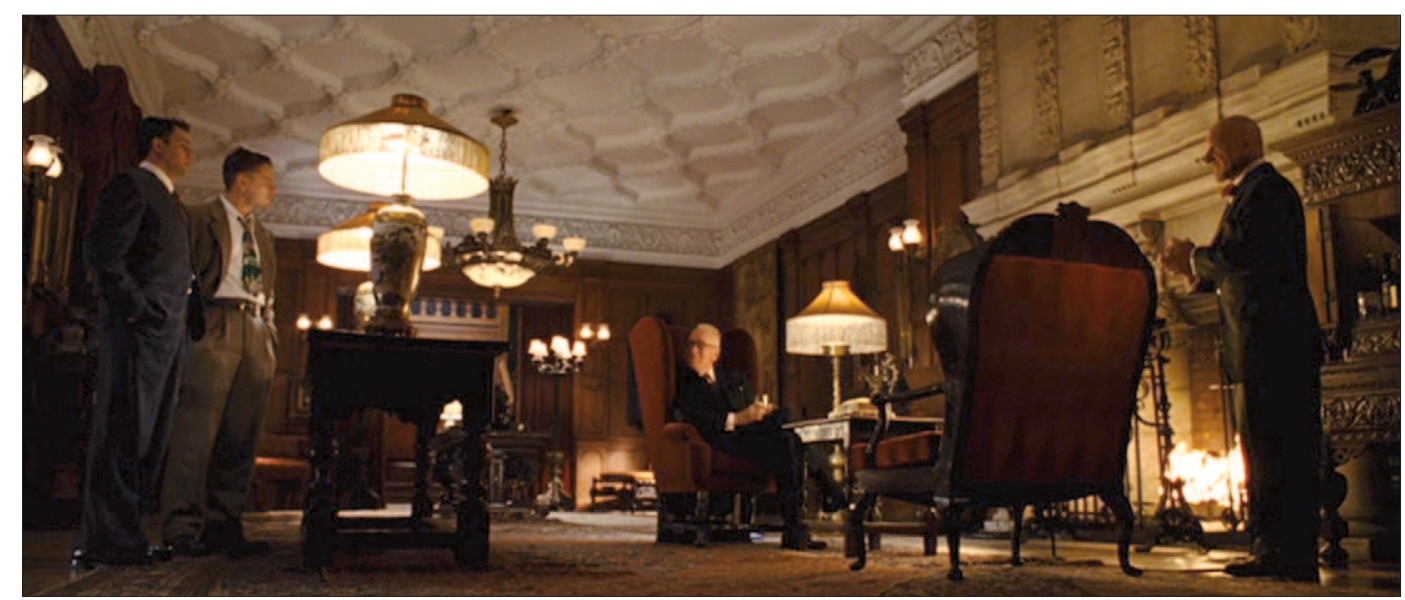

Abb. 1.10 - Totale des Salons Dr. Cawleys, Shutter IsLAnd, Einst. 14, SP 1

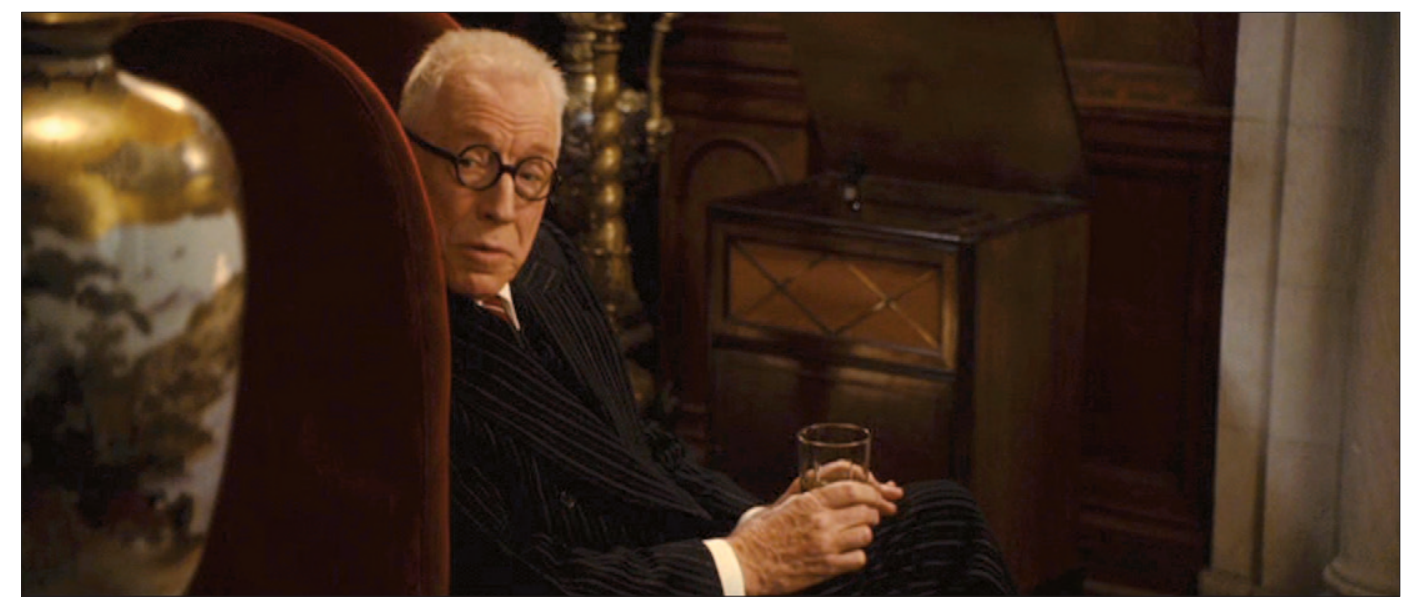

Abb. 1.11 - Dr. Naehring im Salon Dr. Cawleys, Shutter Island, Einst. 11, SP 1 


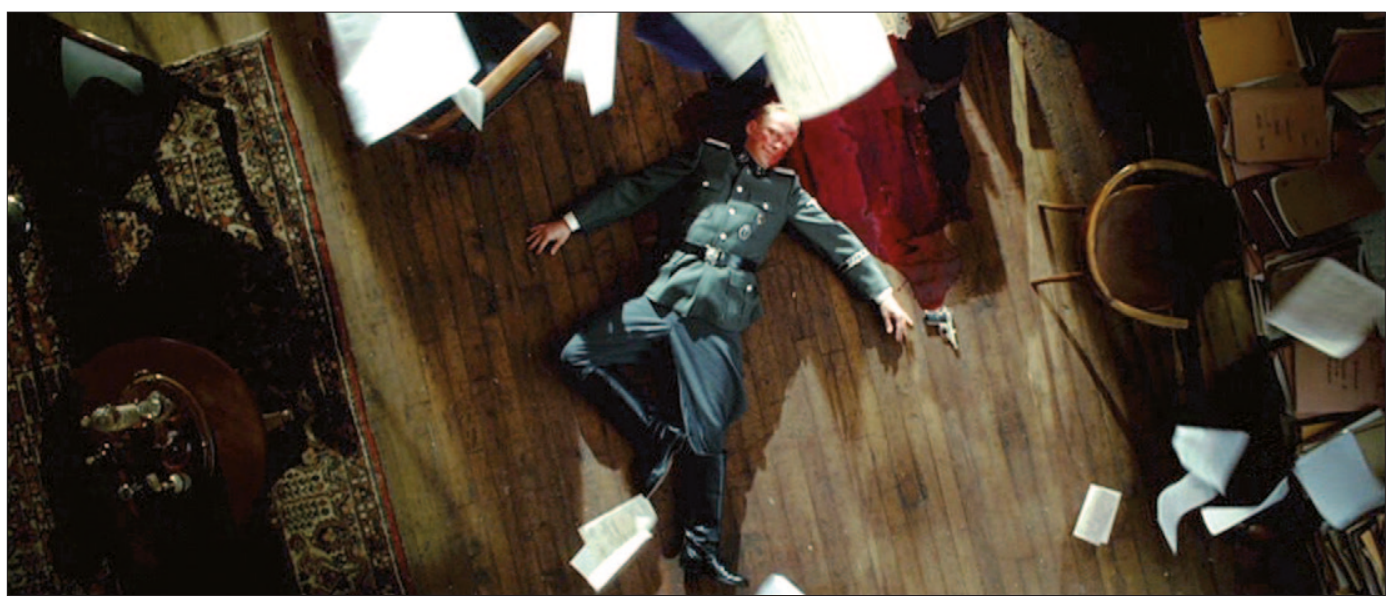

Abb. 1.12 - Vogelperspektive in den Erinnerungen Teddys/Andrews, SHUTter IsLAND, Einst. 43, SP 1

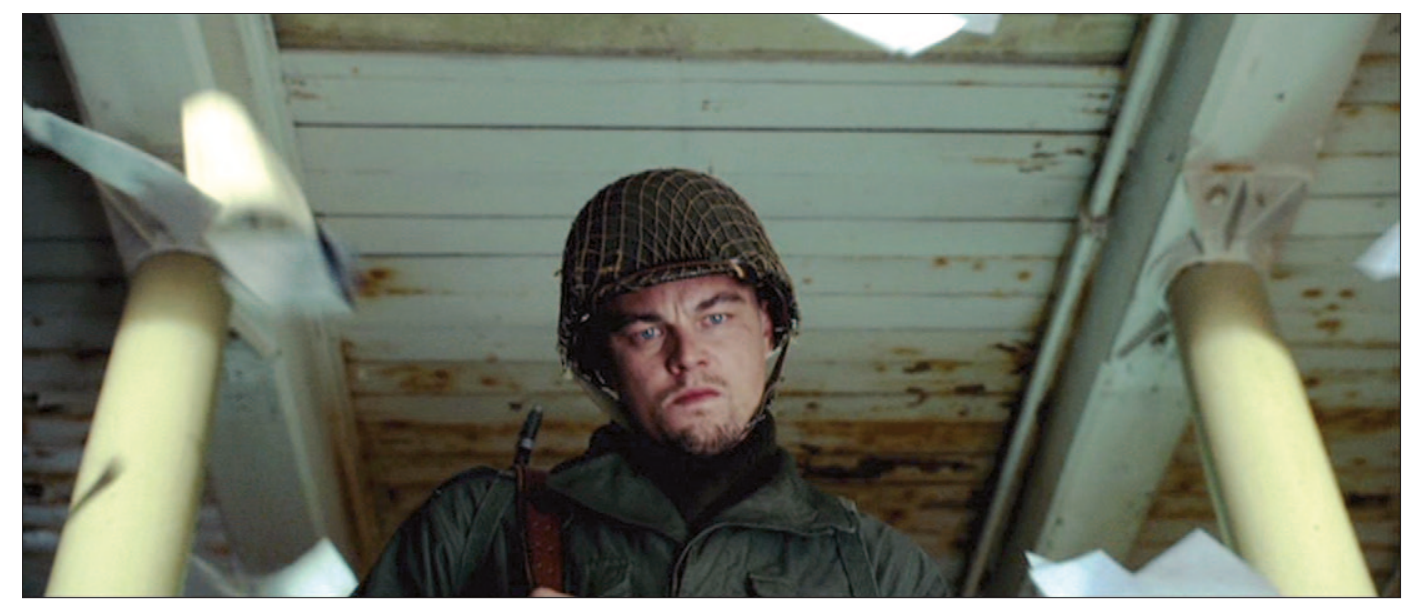

Abb. 1.13 - Froschperspektive in den Erinnerungen Teddys/Andrews, SHUTter IsLAND, Einst. 45, SP 1

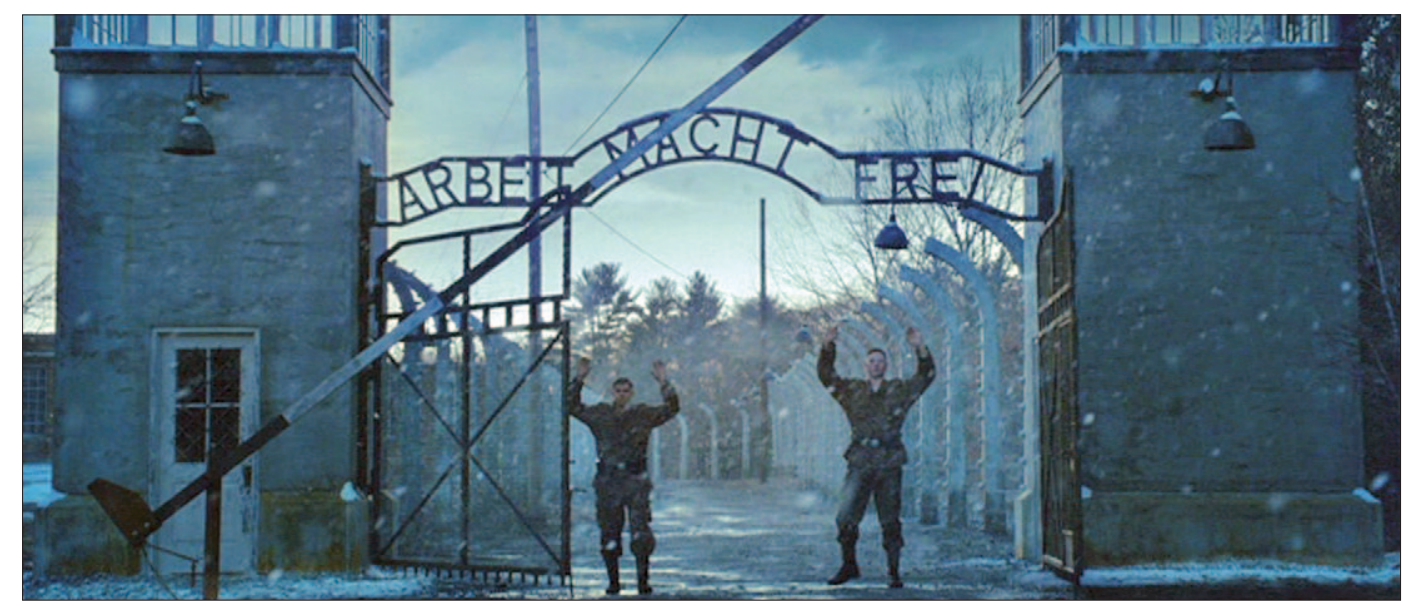

Abb. 1.14 - Tor des KZ Dachau im Film, Shutter IsLAND 


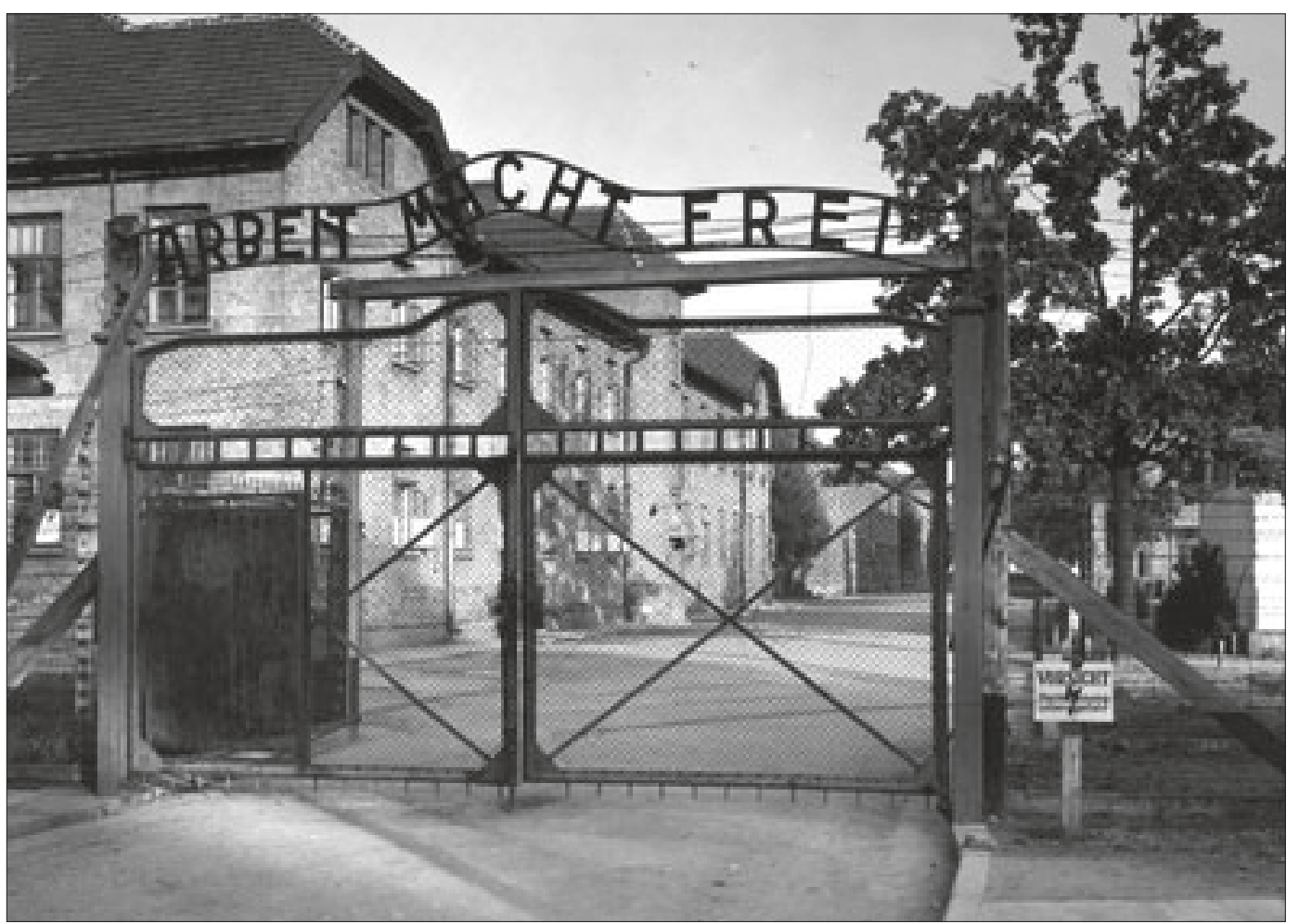

Abb. 1.15 - KZ-Tor Auschwitz, Yad Vashem Photo Archive (5353/250), Stanisław Mucha, http://collections.yadvashem.org/photosarchive/en-us/64935.html (Zugriff: 14.12.2011).

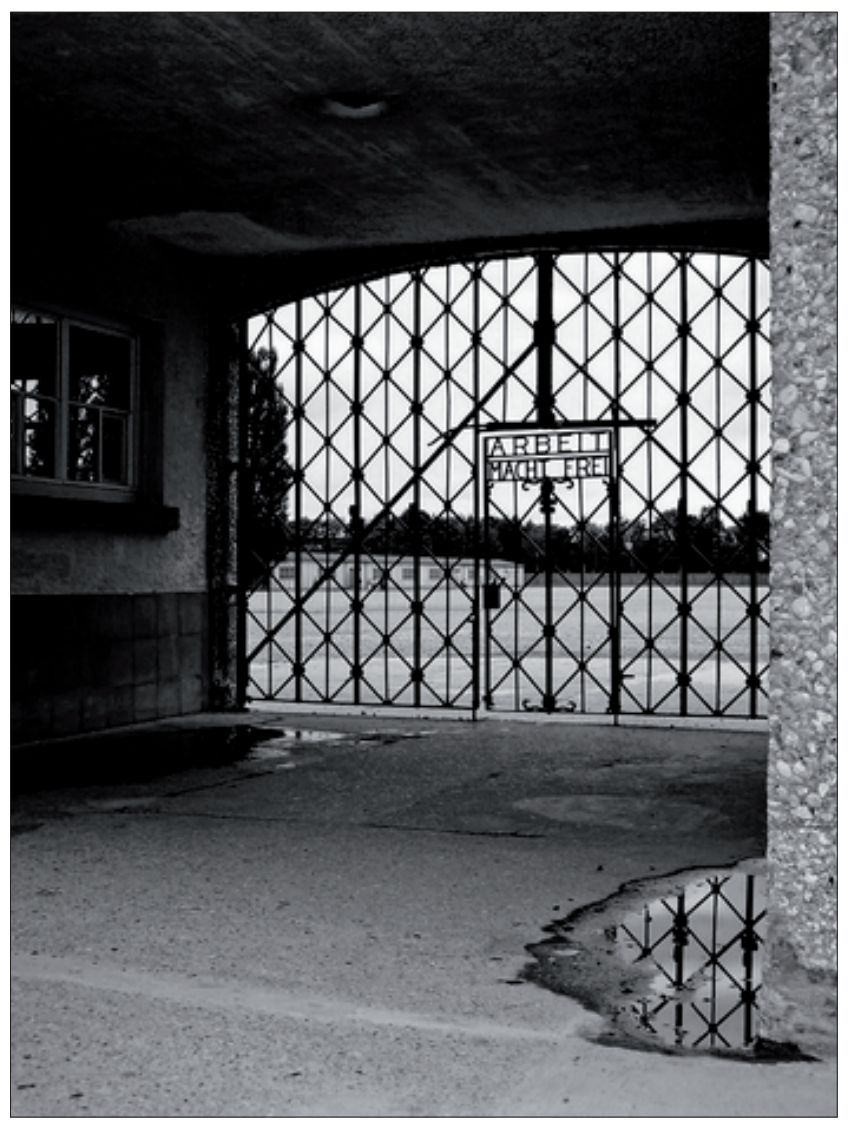

Abb. 1.16 - KZ-Tor Dachau 2005, Yad Vashem Photo Archive (8603/5), Daniel Brunod, http://collections.yadvashem.org/photosarchive/en-us/7323641.html (Zugriff: 14.12.2011). 


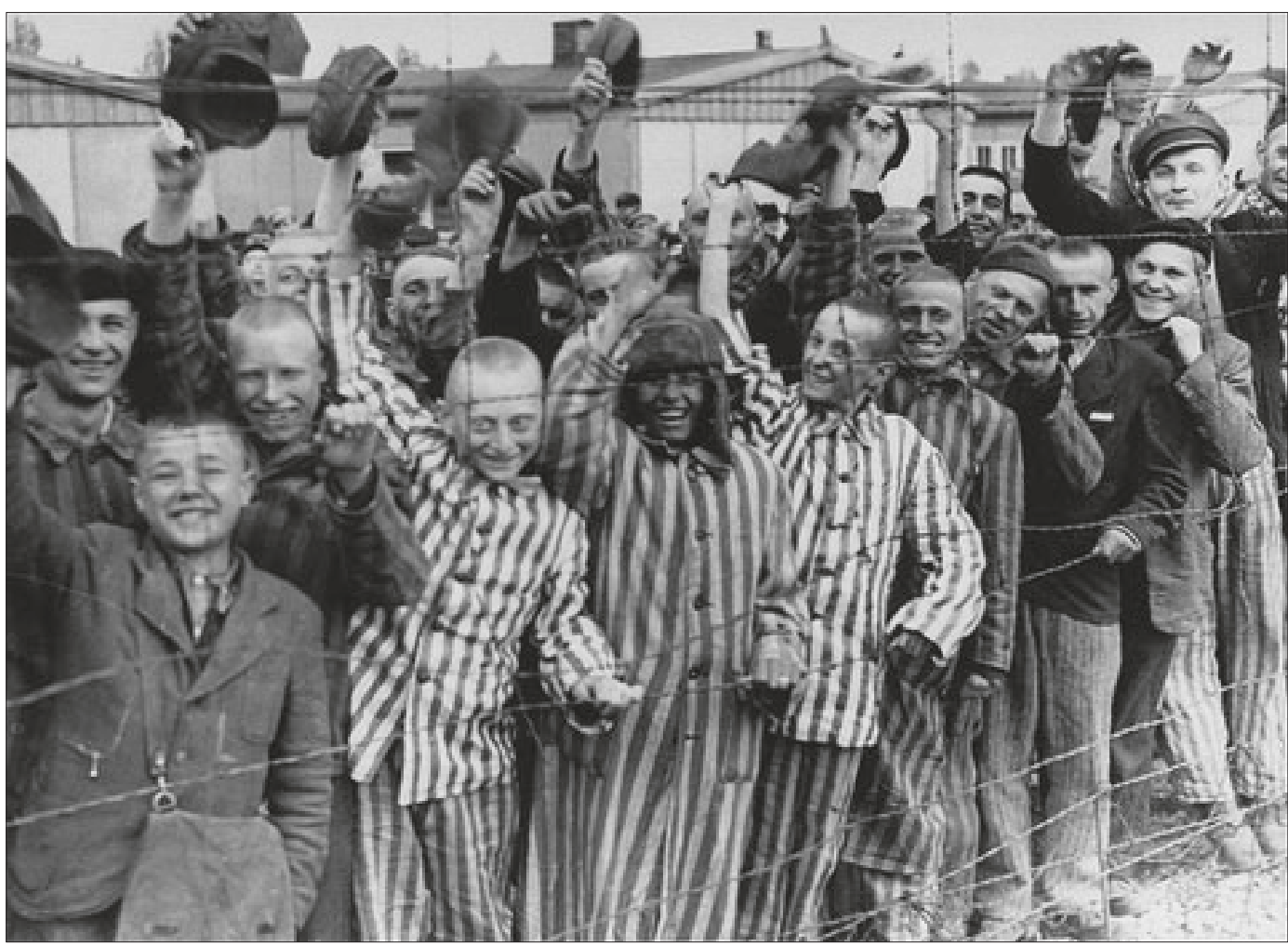

Abb. 1.17 - "Young and old survivors in Dachau cheer approaching U.S. troops. Among those pictured are Juda Kukiela (middle), Tevya Grojs (second from the right), David Moszkowicz (fourth from the left), Szmulek Rozental (third from the left) and Gyorgy Laszlo Spiegel (second from the left).«, 29.04.1945, United States Holocaust Memorial Museum (\#45075), http://resources.ushmm.org/inquery/uia_doc.php/ photos/11497?hr=null (Zugriff: 14.12.2011).

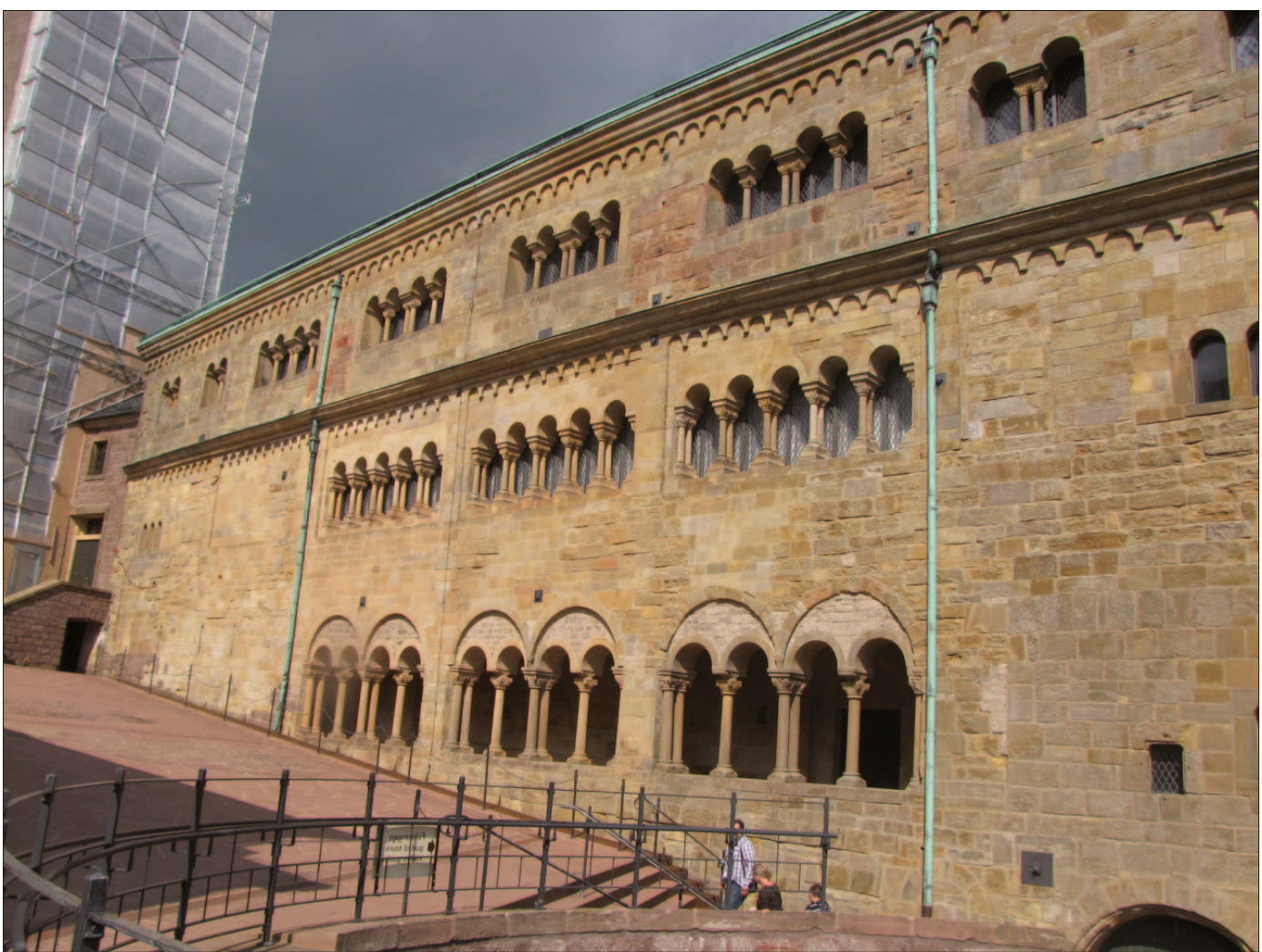

Abb. 1.18 - Palas der Wartburg, Eisenach am 24.05.2010, André Wendler 


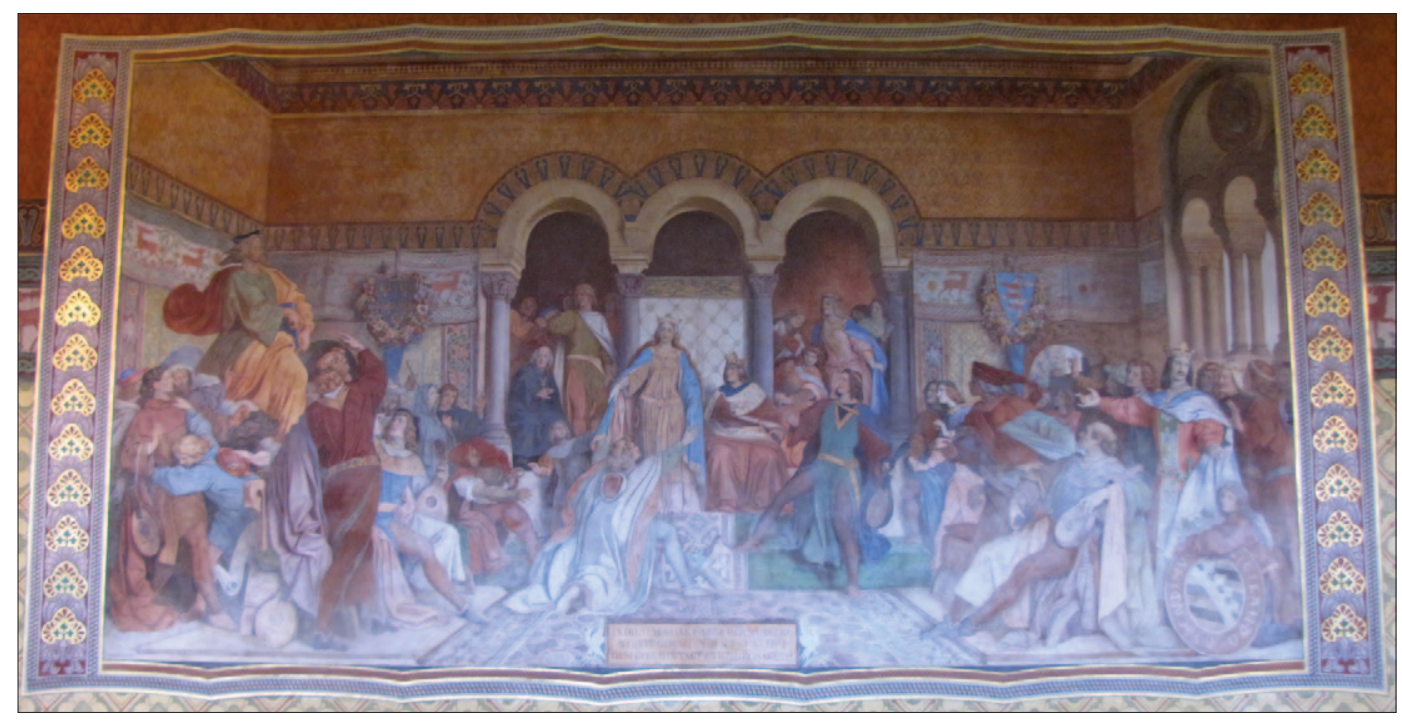

Abb. 1.19 - Moritz von Schwind, Sängerkriegsfresko im Sängersaal der Wartburg, Eisenach (1855) am 24.05.2010, André Wendler

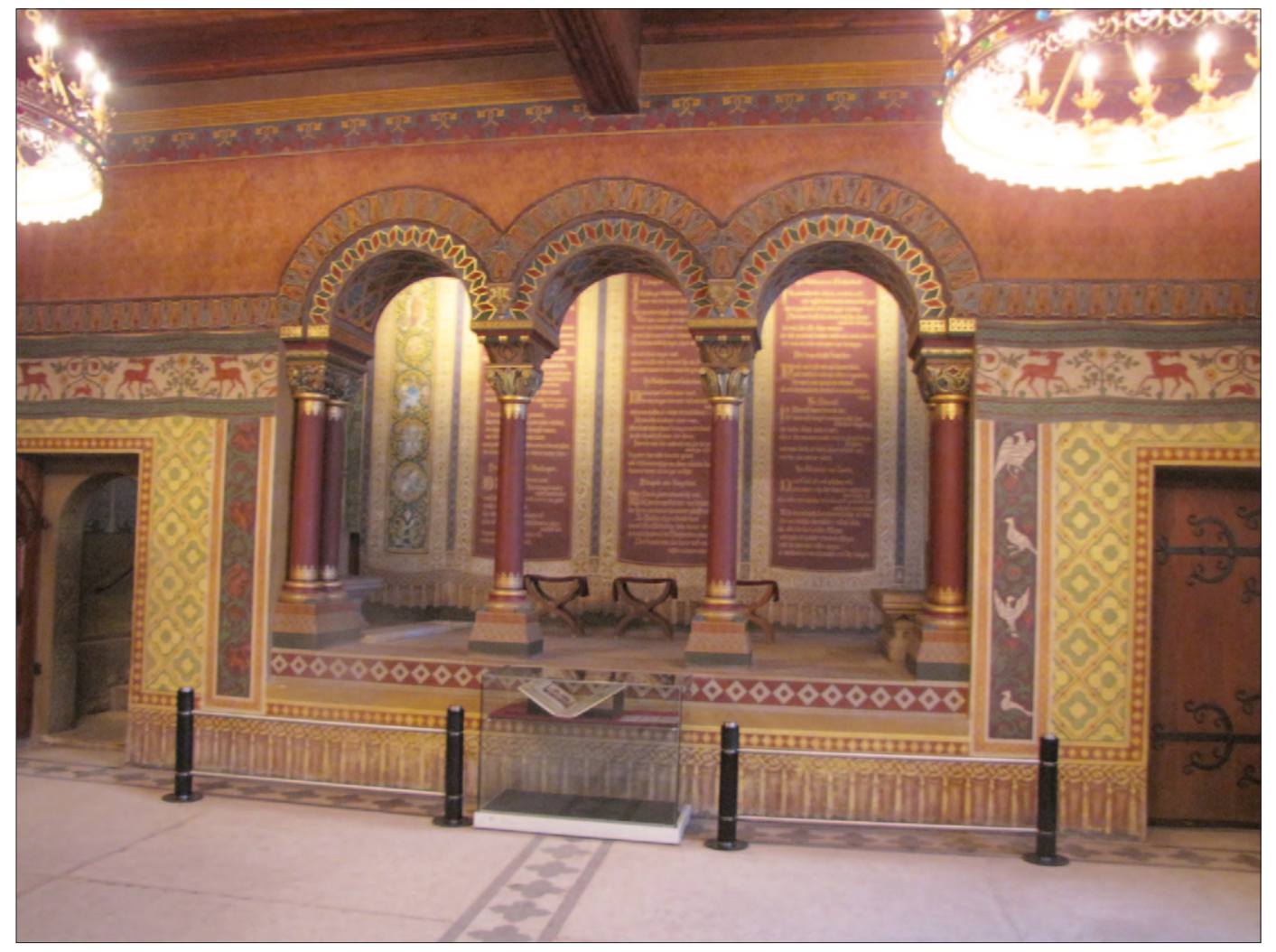

Abb. 1.20 - Sängerlaube im Sängersaal der Wartburg, Eisenach am 06.02.2010, André Wendler 


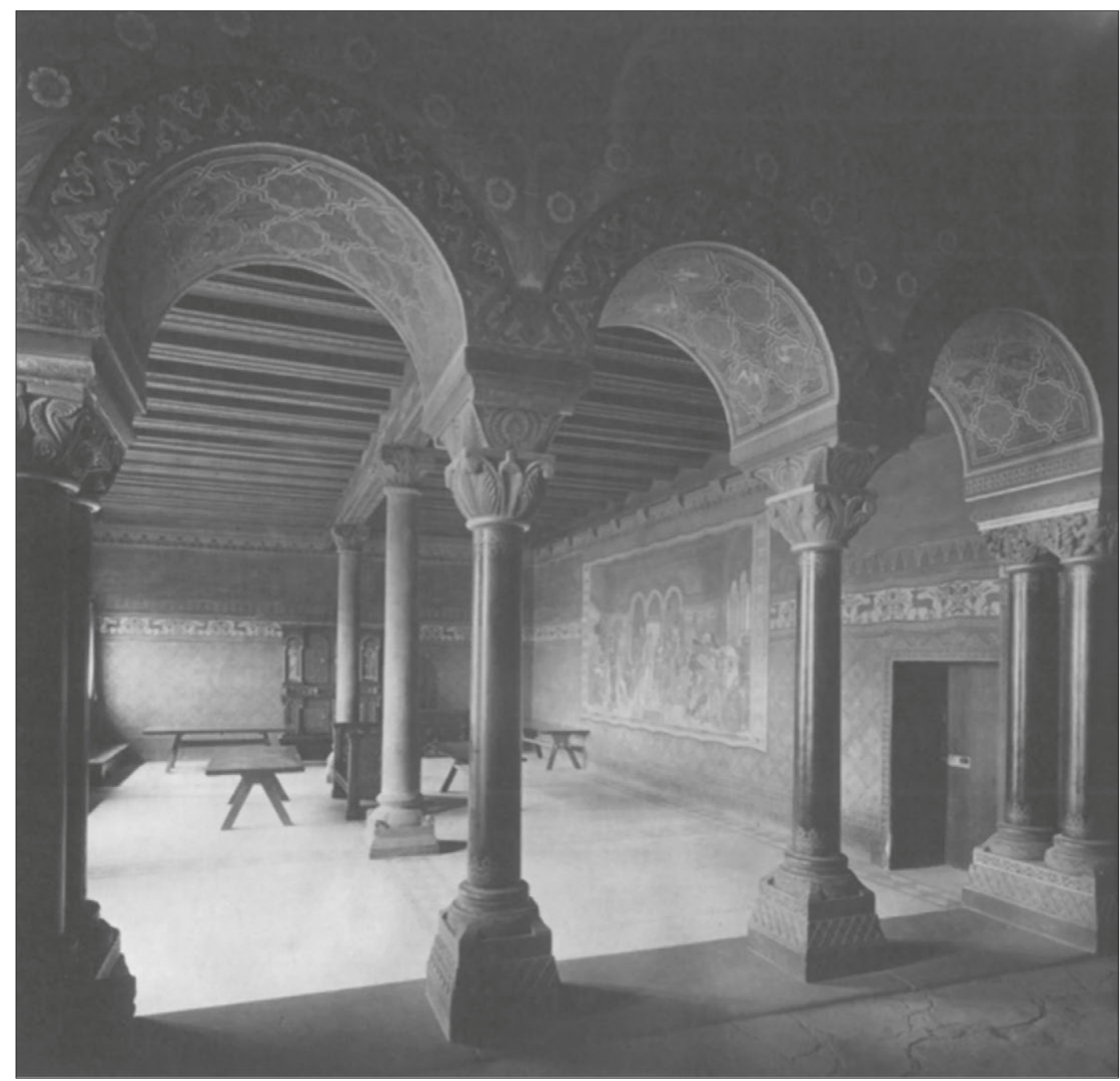

Abb. 1.21 - Foto des Sängersaals der Wartburg aus der Sängelaube mit dem Fresko von Schwinds ca. 1910, Foto der Königlich-Preußischen Messbild Anstalt.

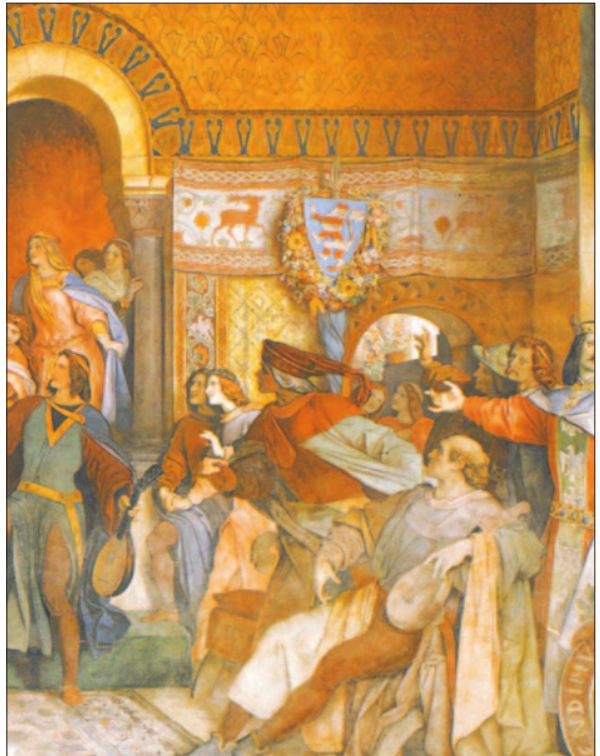

Abb. 1.22 - von Schwind, Sängerkriegsfresko, Detail.

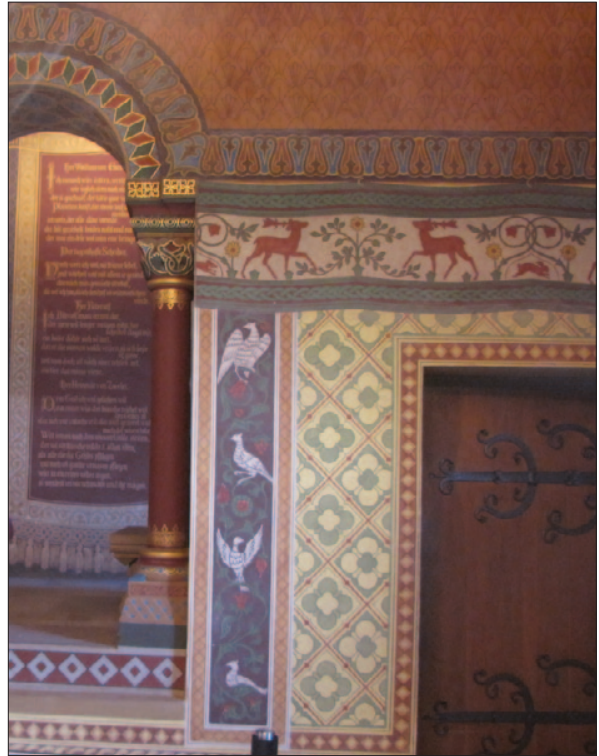

Abb. 1.23 - Detailfotografie des Sängersaals am 24.05.2010, André Wendler 
She wir das Ranografenzimmer verlallen, merfen wir nod) einten Blicf Durdh Die senfter auf bie reizende Randichaft, und Dann treten mir Durch Die vom Römen berwadbte Thür in Den

\section{Süttgerjạt.}

Diefer Saal mar der uriprüngliche feftiaal, in meldfem auch) bie Did̄ter ifre (seänge auffübrten; von Dem Sand= grafenzimmer bis zum füblichen (5iebel des ßalajtes reichend, bildete er ein Ganzes, von bem nur ein fleiner Theil über Der aufiteigenten Treppe Durd) eine offene Bogenftelfung in

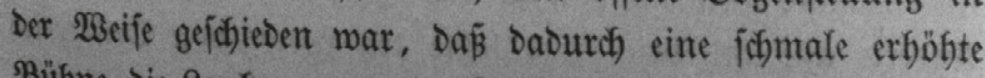
Bühne, bie Laube genannt, gefondert murbe, und bieje Laube

Abb. 1.24 - Detail aus von Ritgen, 1859/1876, Der Führer auf der Wartburg, S. 115.

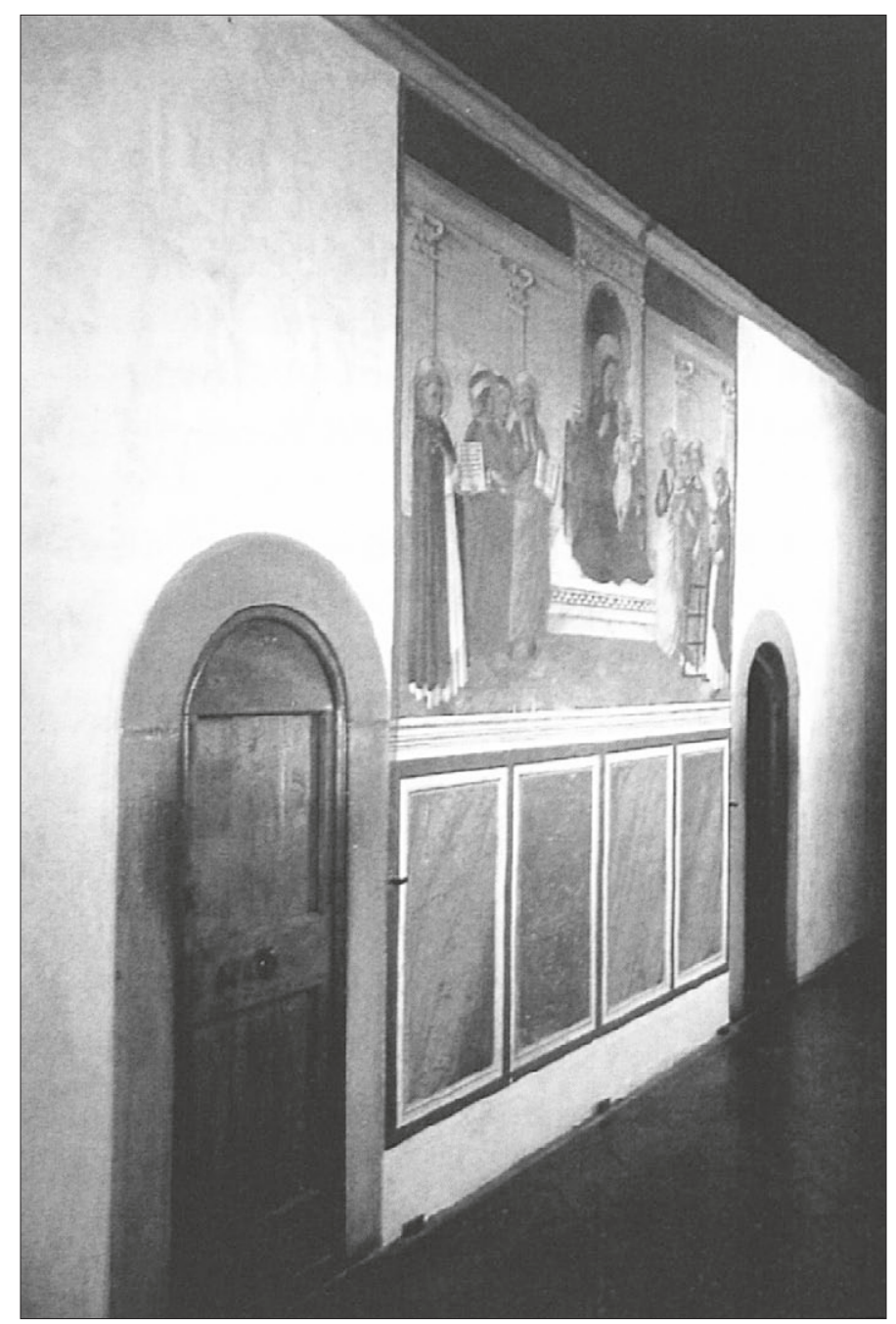

Abb. 1.25 - Fra Angelico, Sacra Conversazione, genannt Die Madonna der Schatten. Zwischen 1438 und 1450. Fresko und Tempera. Kloster San Marco, Ostkorridor, Florenz. nach Didi-HubermAN, 1990/1995, Fra Angelico, S. 32, Abb. 3. 


\section{Abbildungen Kapitel 2}

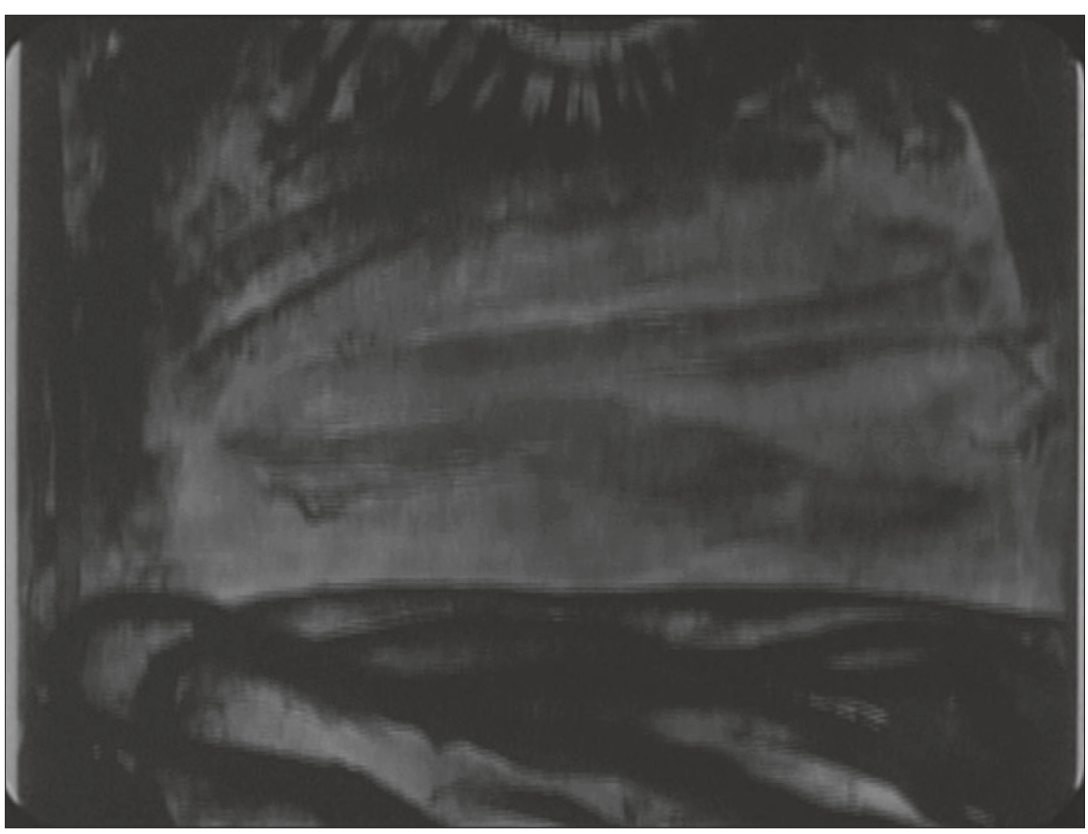

Abb. 2.1 - Anfang von Einst. 86, Chronik der AnNa Magdalena $\mathrm{BACH}$

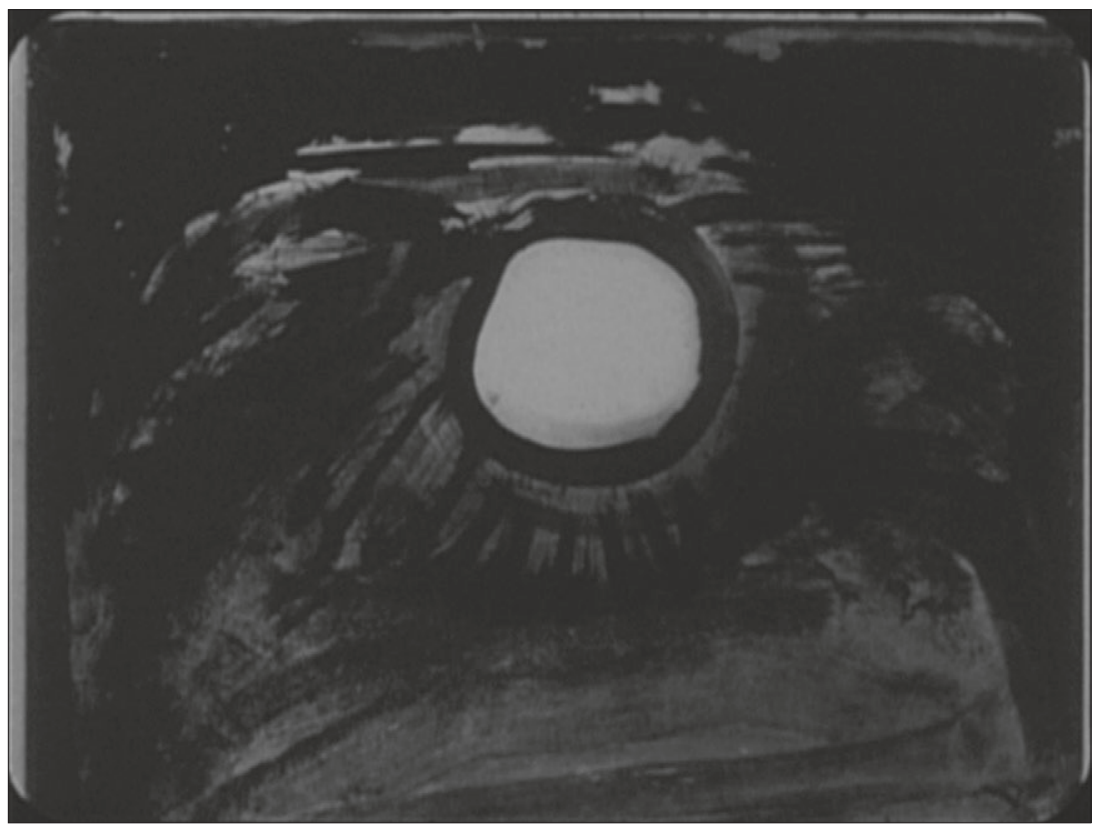

Abb. 2.2 - Ende von Einst. 86, Chronik Der AnNa MagdalenA $\mathrm{BACH}$ 


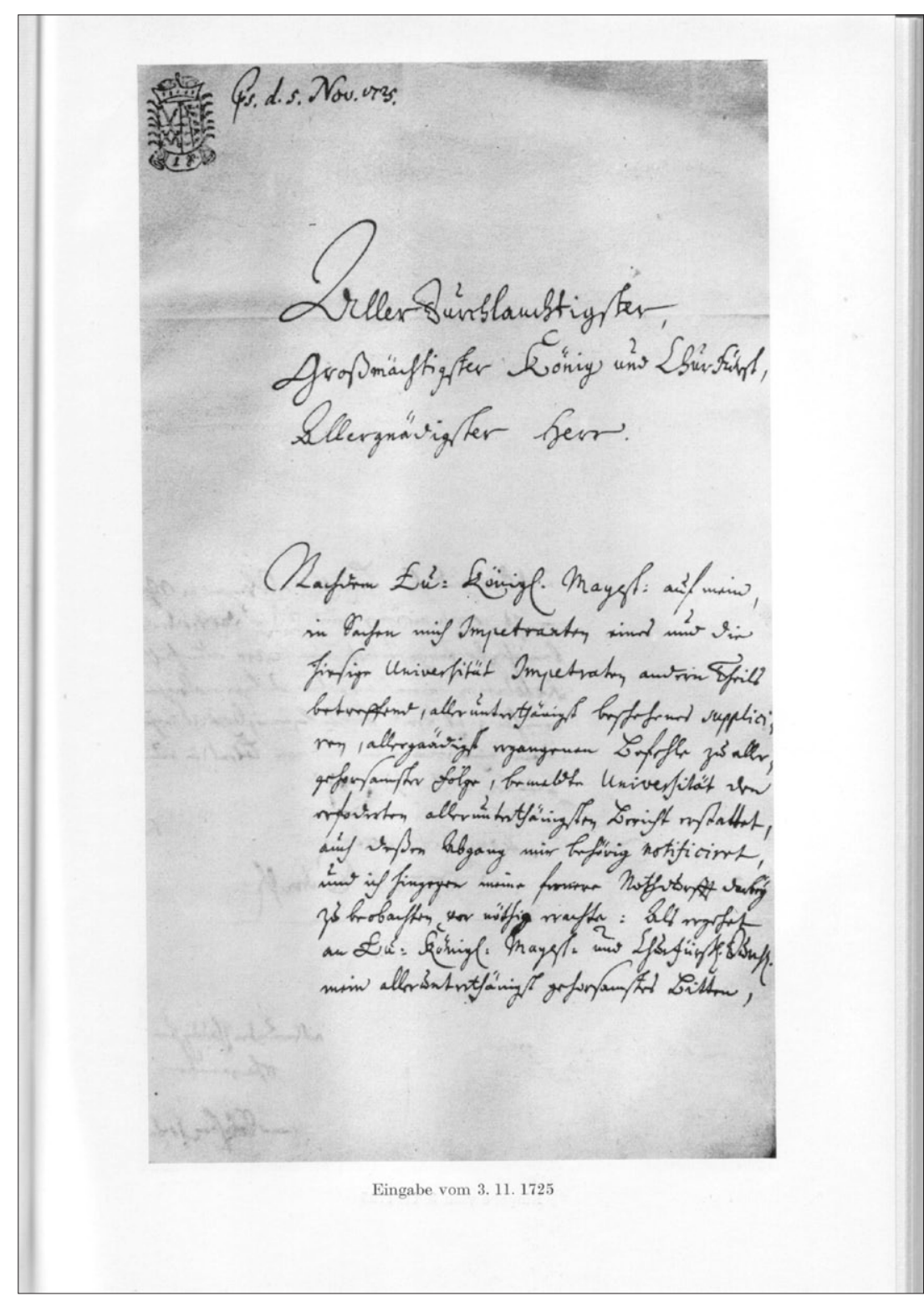

Abb. 2.3 - Bach-Dokumente I, Buchseite mit einem Faksimile nach S. 176 
vien, Samogitien, Kiovien, Volhinien, Podolien, Podlachien,

25 Lieffland, Smolenskien, Severien und Zschernicovien etc. etc. Hertzog zu Sachsen, Jülich, Cleve und Berg, auch Engern und Westphalen; Des Heiligen Röm: Reichs Ertz-Marschalln und ChurFürsten, LandGraffen in Thüringen, MargGrafen zu Meisen, auch Ober- und NiederLausitz, Burg-Grafen zu Magdeburg, Gefürsteten Grafen zu Henneberg, so Grafen zu der Marck, Ravensberg und Barby, Herrn zu Ravenstein etc. Meinem allergnädigsten König, ChurFürsten und Herrn etc.

A. Autograph. 1 Bogen, S. 1, 2 und 4 von Bach beschrieben (S. 3 Aktennotiz), Blattformat $33,5 \times 20,5 \mathrm{~cm}$; WZ: SW. Bl. 1 R. r. o.: Stempel (,Stempelpapier“), daneben: ps. d. 5. Nov. 1725. Bl. 2 V. m. l. und r. je ein Siegelrest. - Universitätsarchiv Leipzig, Rep. II Kap. III Litt. B. Sect. I No. 22 (ACTA Das von dem Cantore zu St. Thomas Johann Sebastian Bachen gesuchte Salarium vom Neuen Gottesdienste in der Pauliner Kirche betr. de Anno 1725).

Textdr.: Spitta A II, S. 41; engl.: Spitta B II, S. 217f.; Terry, S. 183; Bach Reader, S. $99 \mathrm{f}$; ; franz.: Prod'homme, S. $265 \mathrm{f}$; ; span.: Spitta C, S. 199

B. 4, 10, 16) Königl . 10, 17) ChurFürstl .; Durchl . 27) Heil . 29) Heñeberg.

C. Laut einer Aktennotiz vom 30. 10. 1725 hatte der Pedell Johann Ackermann Bach mitgeteilt, da $\mathrm{B}$ am folgenden Tage ein Berieht ins Oberkonsistorium abgehen werde. Anscheinend wurde der Streitfall (über die Vorgeschichte vgl. Dok. 10, über den Fortgang Dok. 12) dem Landesherrn und der Kirchenbehörde gleichzeitig unterbreitet. Bei dieser Gelegenheit dürfte Bach auch von dem an Friedrich August I. gerichteten Schreiben der Universität vom 29.10. 1725 (abgedruckt unter Dok. 12) erfahren haben, um dessen abschriftliche Mitteilung er im vorliegenden Briefe bittet.

Lit.: Spitta A II, S. 41 f.

12.

Eingabe aN KuRFürst Friedrich AUGUSt I. von Sachsex

LeIrzig, 31. 12. 1725

$\left[1^{r}\right]$

AllerDurchlauchtigster, Großmächtigster

König und ChurFürst

Allergnädigster Herr.

Daß Ew: Königliche Majestät und Churfürstliche Durchlaucht mir von dem-

5 jenigen was die Universitat alhier auf meine wegen des Directorii Musices bey dem alten und neuen Gottesdienst in der Pauliner Kirche und des zu dem erstern gehörigen, bißher verweigerten Salarii wieder sie geführte Beschwerde darwieder eingewendet Abschriefft ertheilen zu laßen allergnädigst geruhen

Abb. 2.4 - Bach-Dokumente I, S. 34 


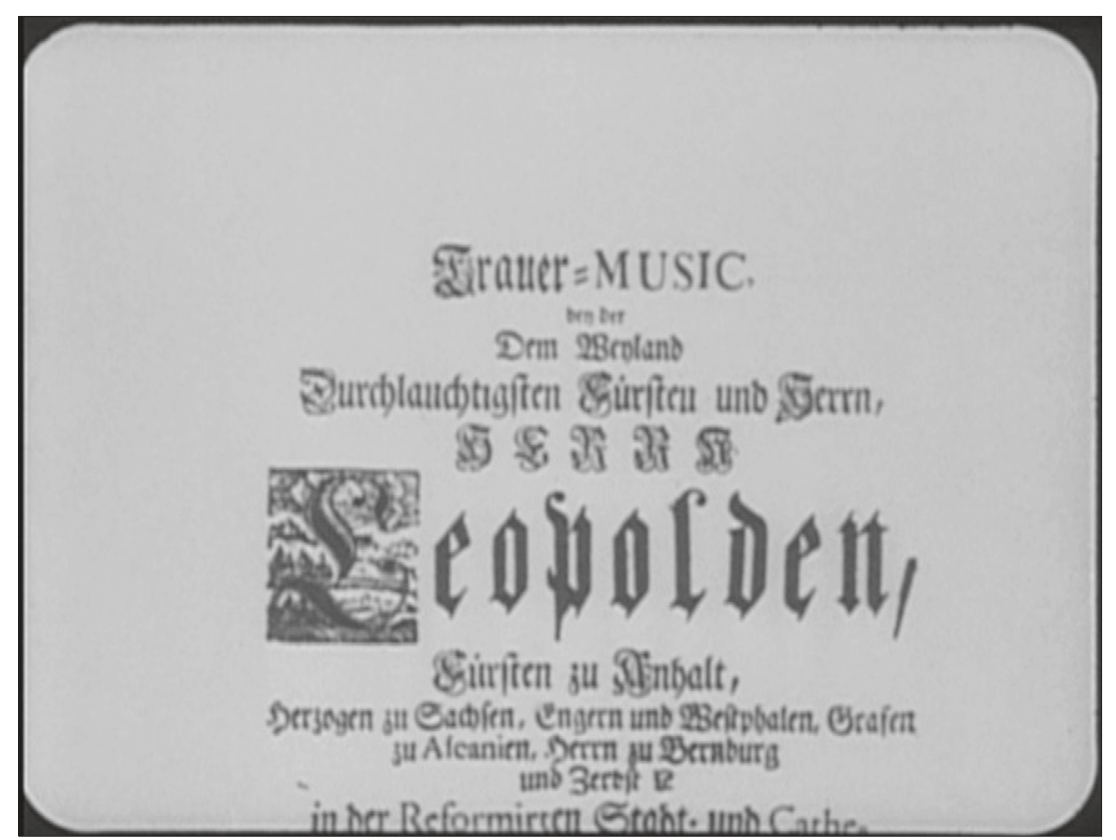

Abb. 2.5 - Anfang von Einst. 40, CHRONiK Der AnNA Magdalena $\mathrm{BACH}$ 


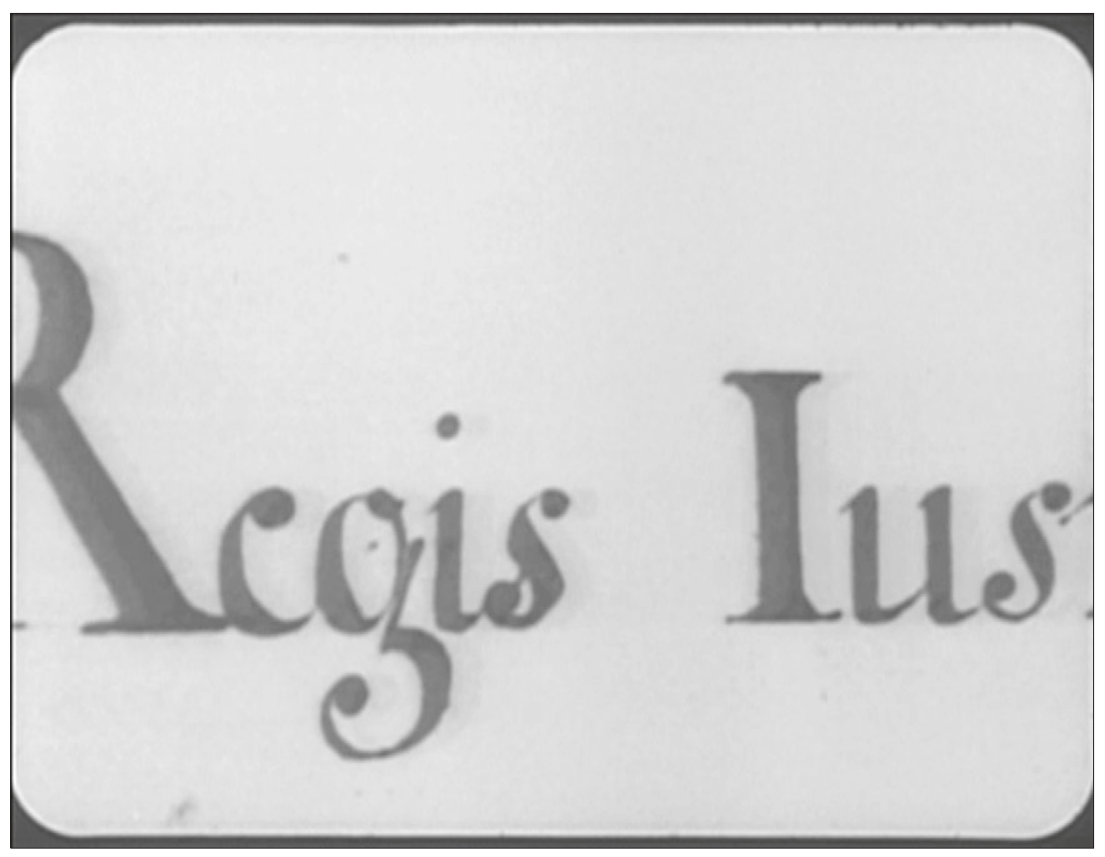

Abb. 2.6 - Anfang von Einst. 97, CHRONiK DER ANNA MagdalenA $\mathrm{BACH}$

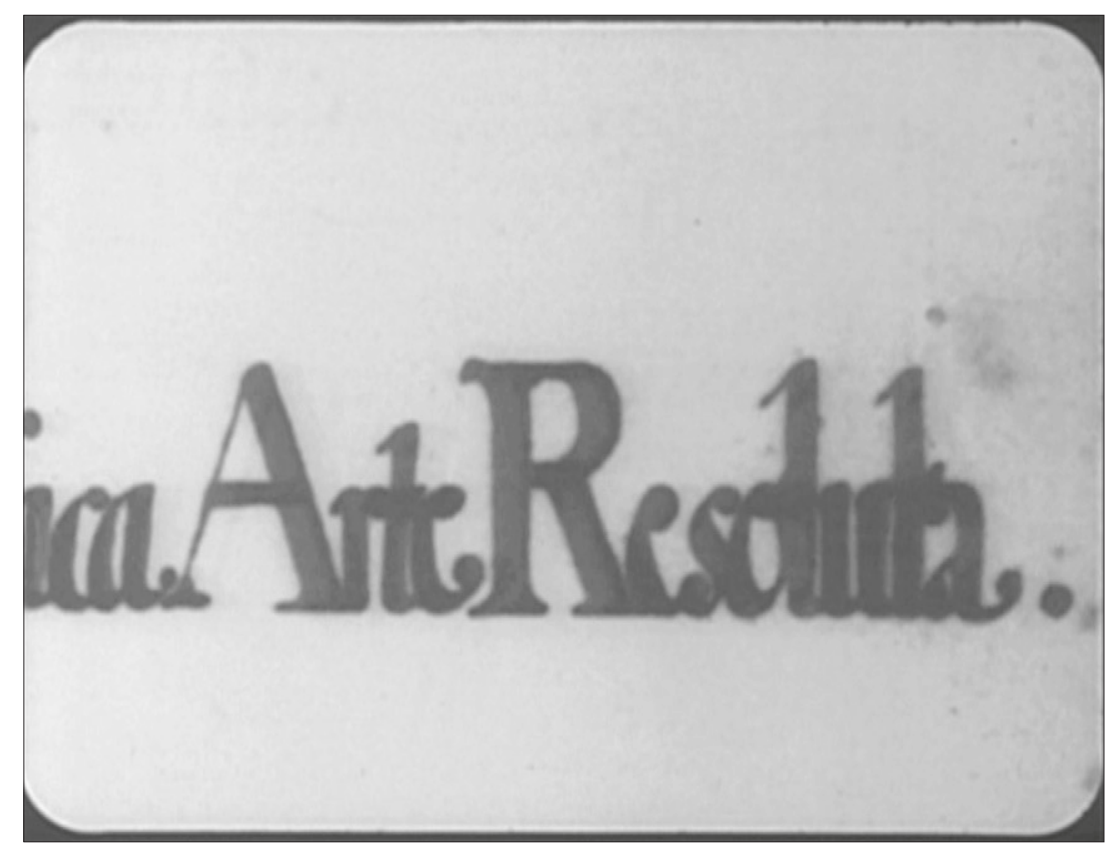

Abb. 2.7 - Ende von Einst. 97, ChroniK der AnNa Magdalena $\mathrm{BACH}$ 


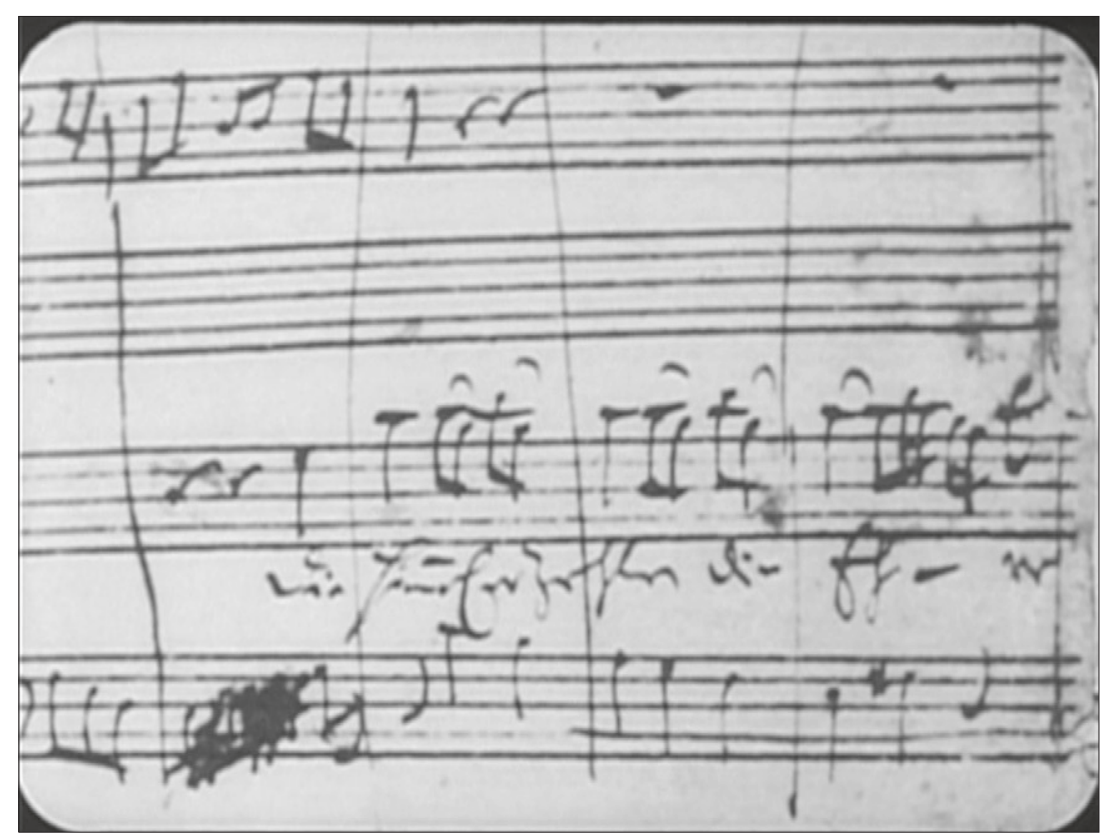

Abb. 2.8 - Einst. 17, ChroniK DeR AnNA MagdalenA BACH

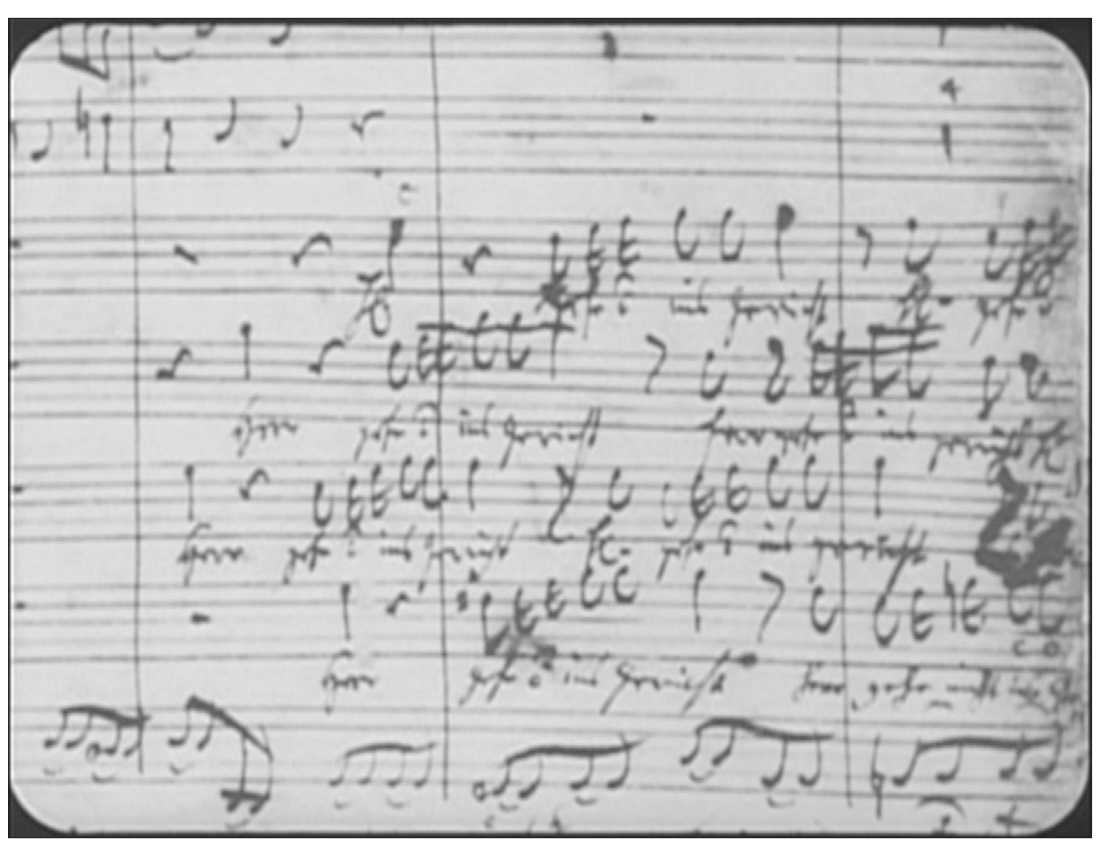

Abb. 2.9 - Einst. 22, CHRONIK DER ANNA MAgDAlenA BACH 


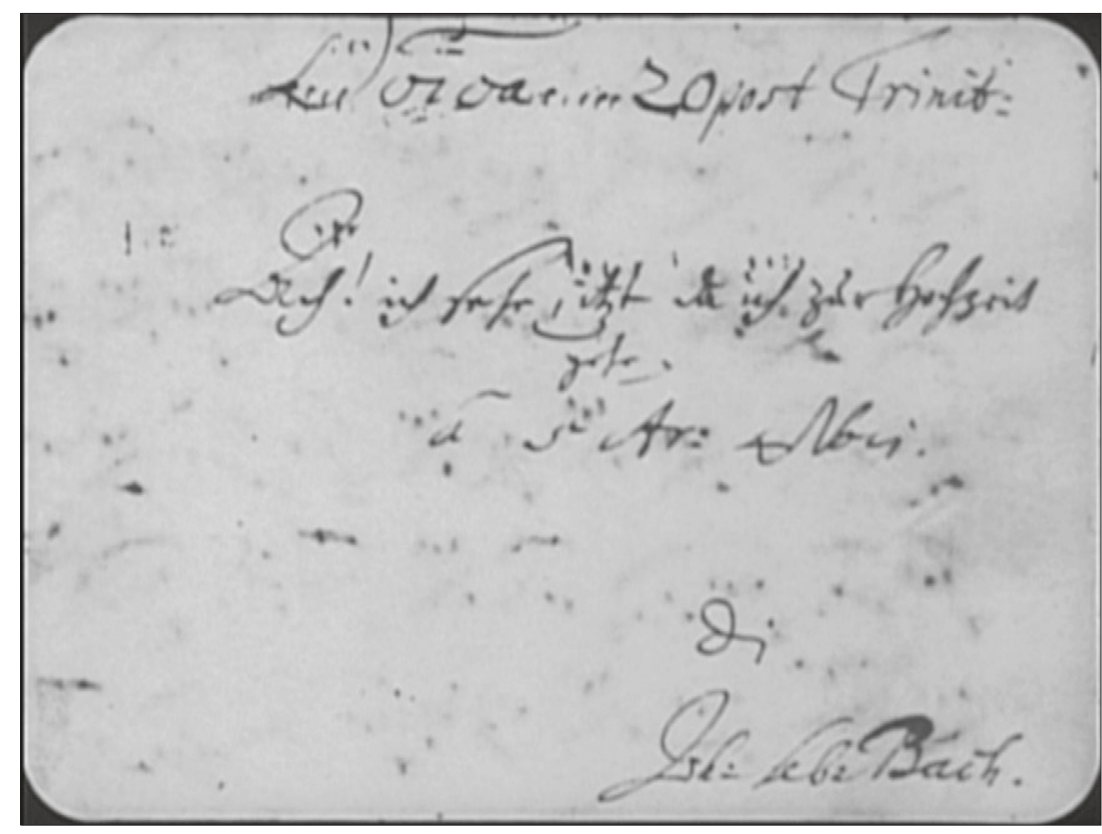

Abb. 2.10 - Einst. 28, CHRONIK DER ANNA MAgdalenA BACH

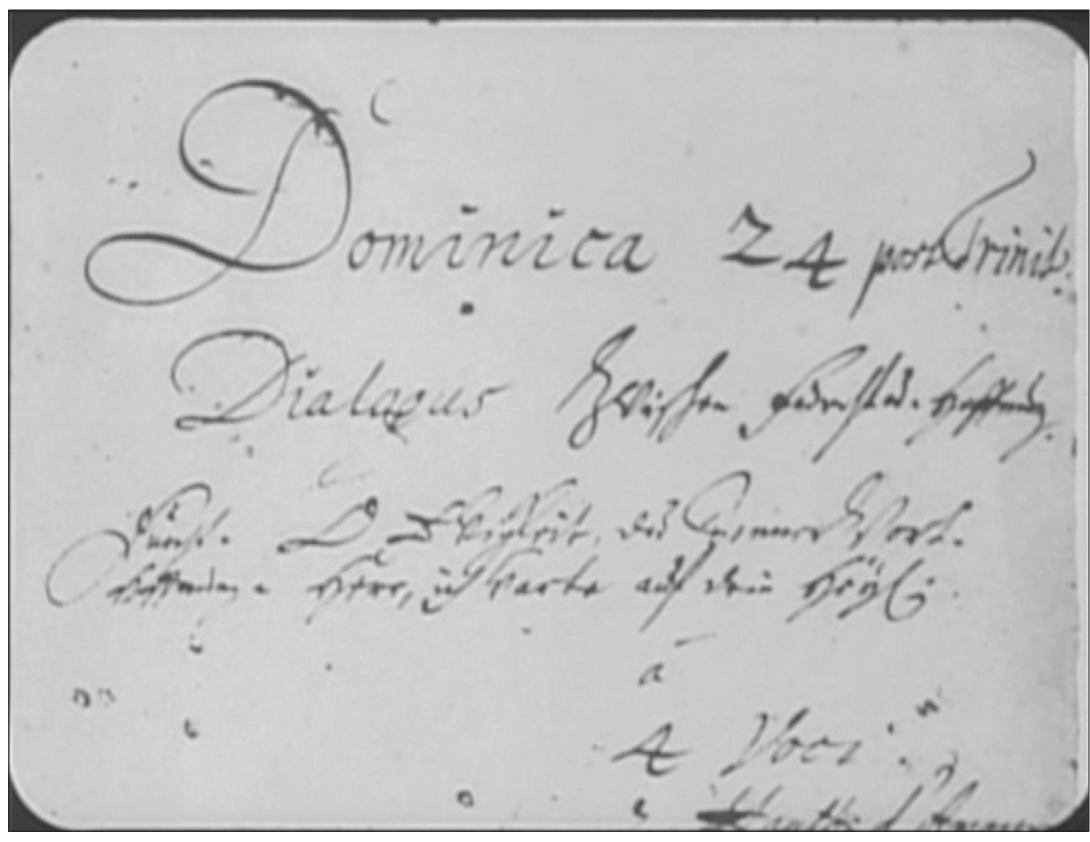

Abb. 2.11 - Einst. 30, Chronik Der AnNA Magdalena BACH 


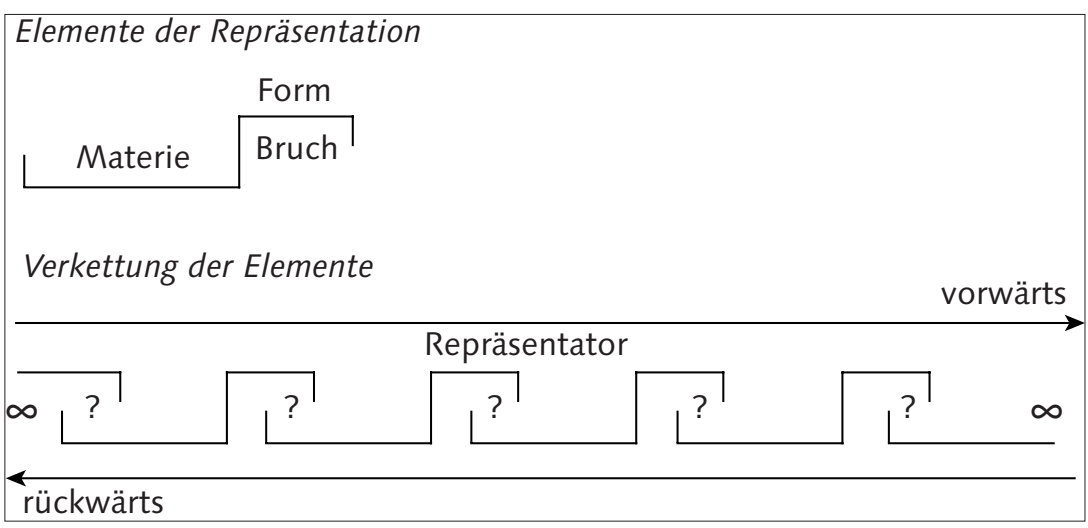

Abb. 2.12 - Zirkulierende Referenz nach Bruno Latour, 1999/2000, Die Hoffnung der Pandora. Frankfurt am Main, S. 85, Abb. 2.21

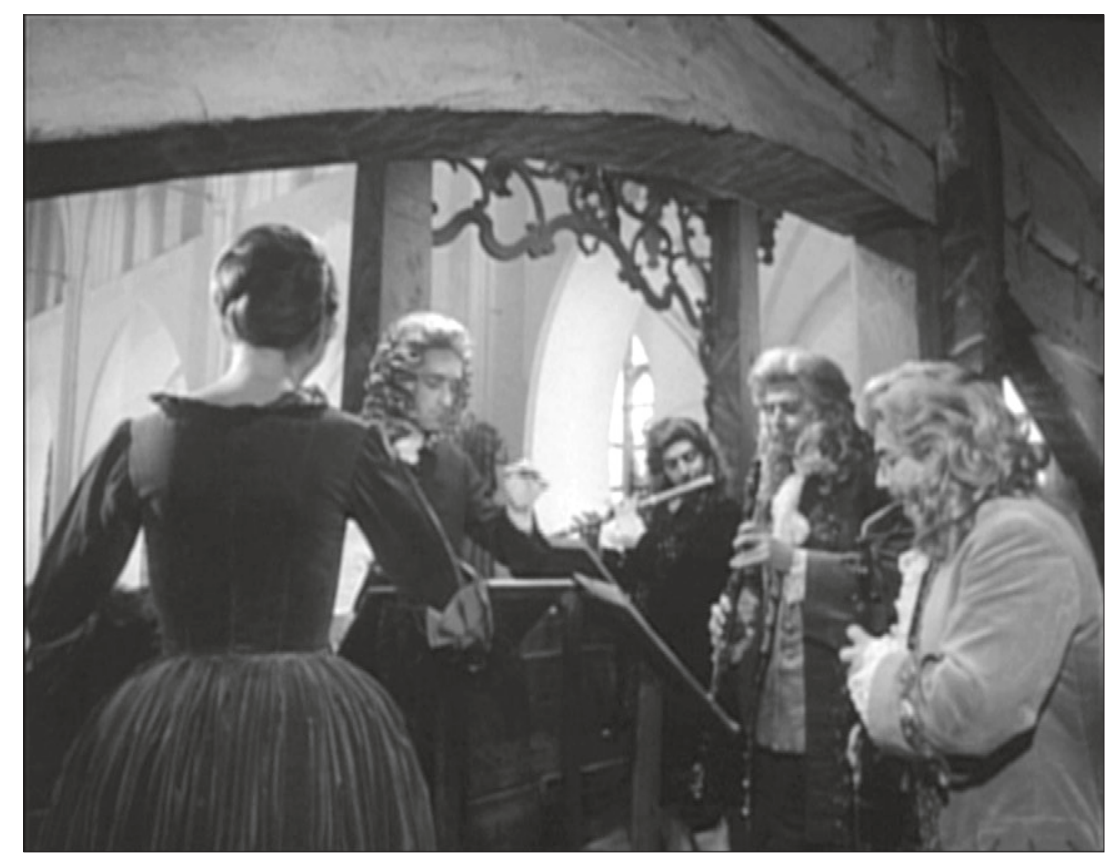

Abb. 2.13 - Einst. 41, Chronik der Anna Magdalena Bach 


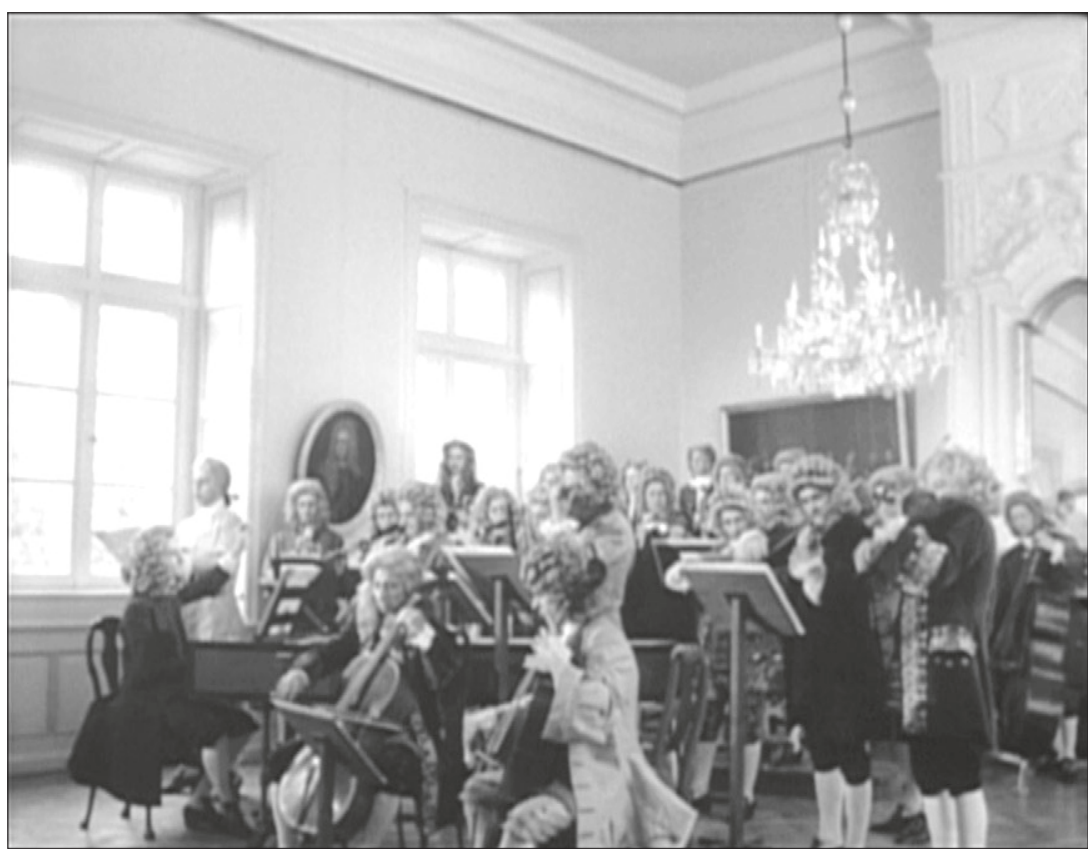

Abb. 2.14 - Anfang von Einst. 37, CHRONiK der ANNA MagdalenA $\mathrm{BACH}$

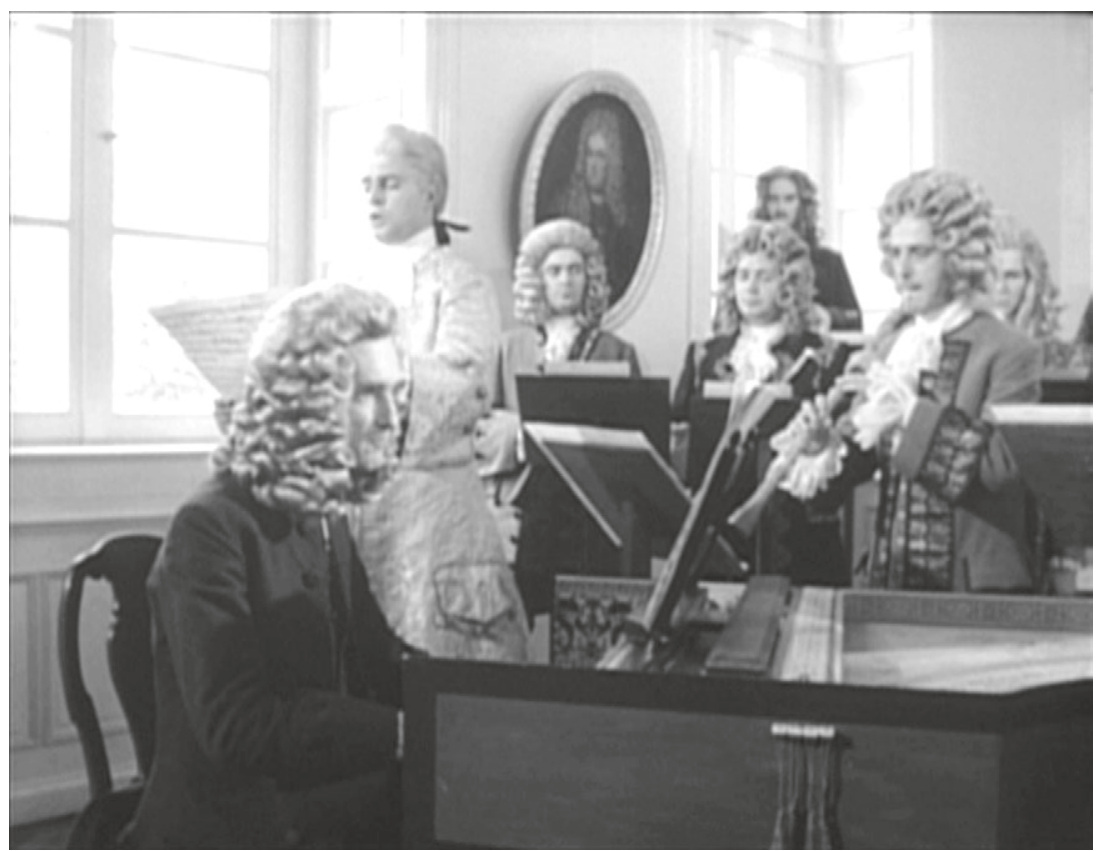

Abb. 2.15 - Ende von Einst. 37, CHRONIK DER ANNA MAgdALENA $\mathrm{BACH}$ 

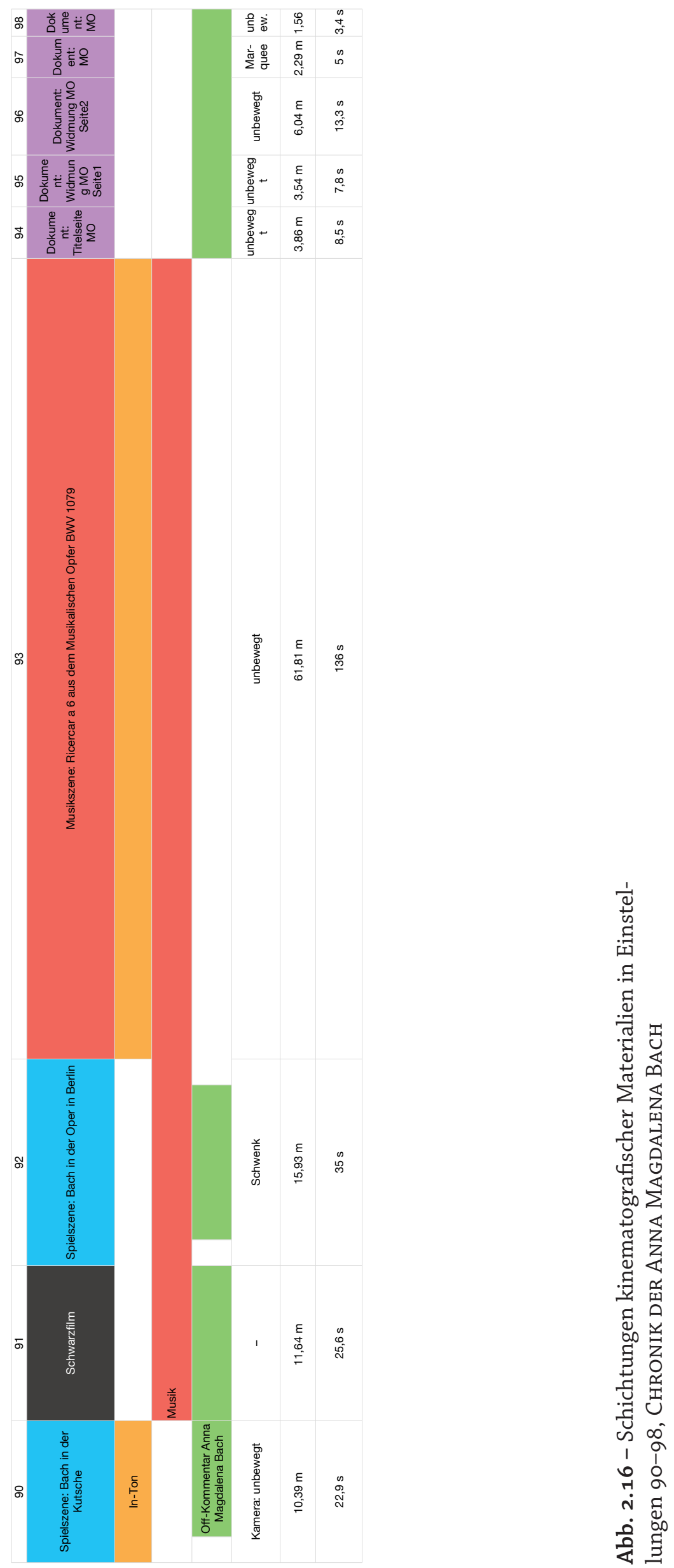
Abbildungen Kapitel 3

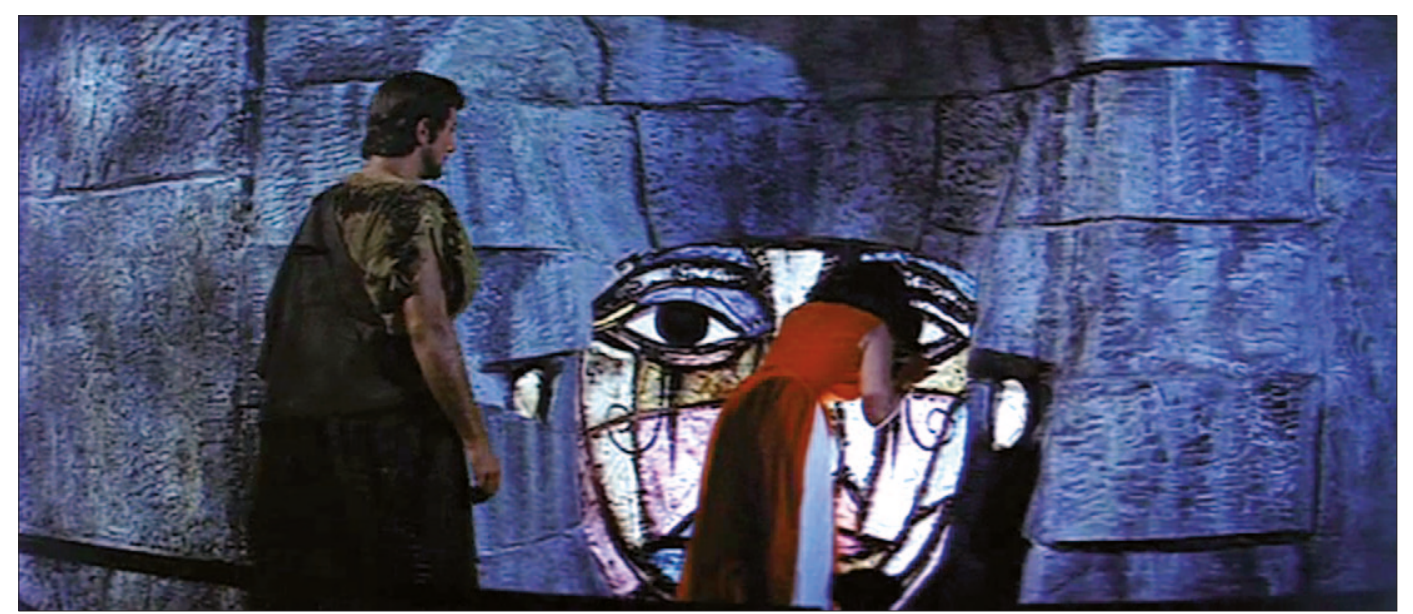

Abb. 3.1 - Einst. 1 nach Sequenzprotokoll 3, CLEOPATRA

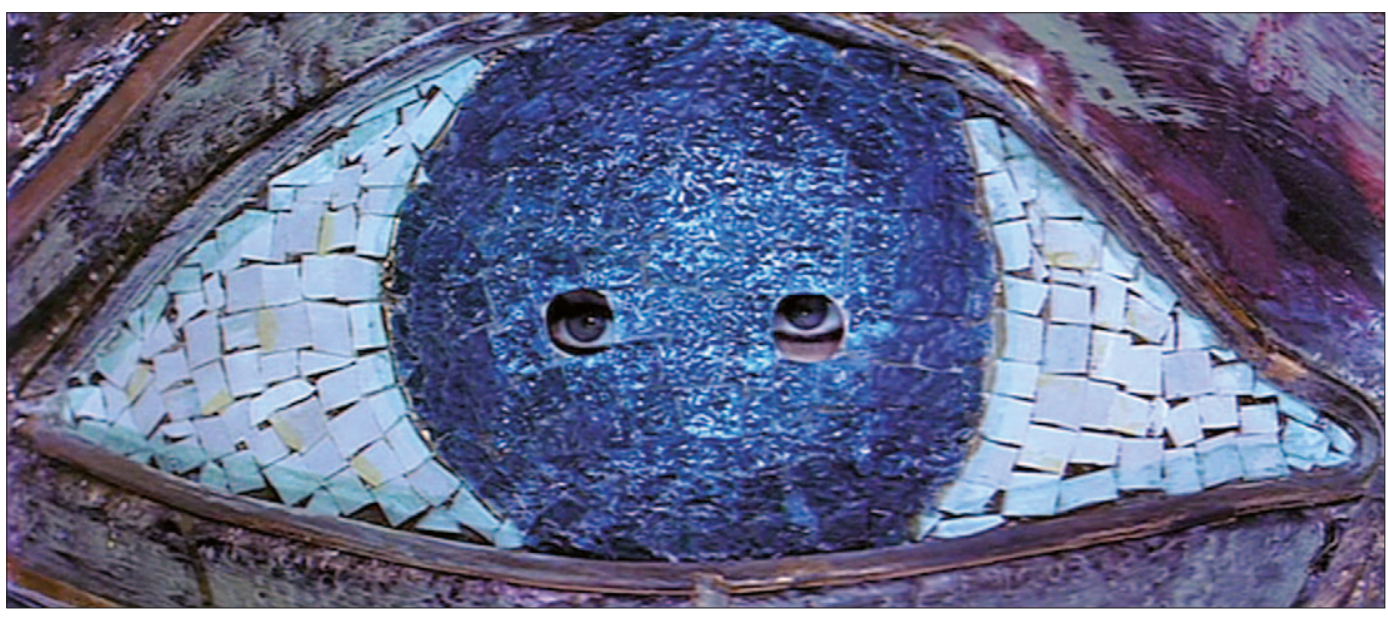

Abb. 3.2 - Einst. 2 nach SP 3, Cleopatra

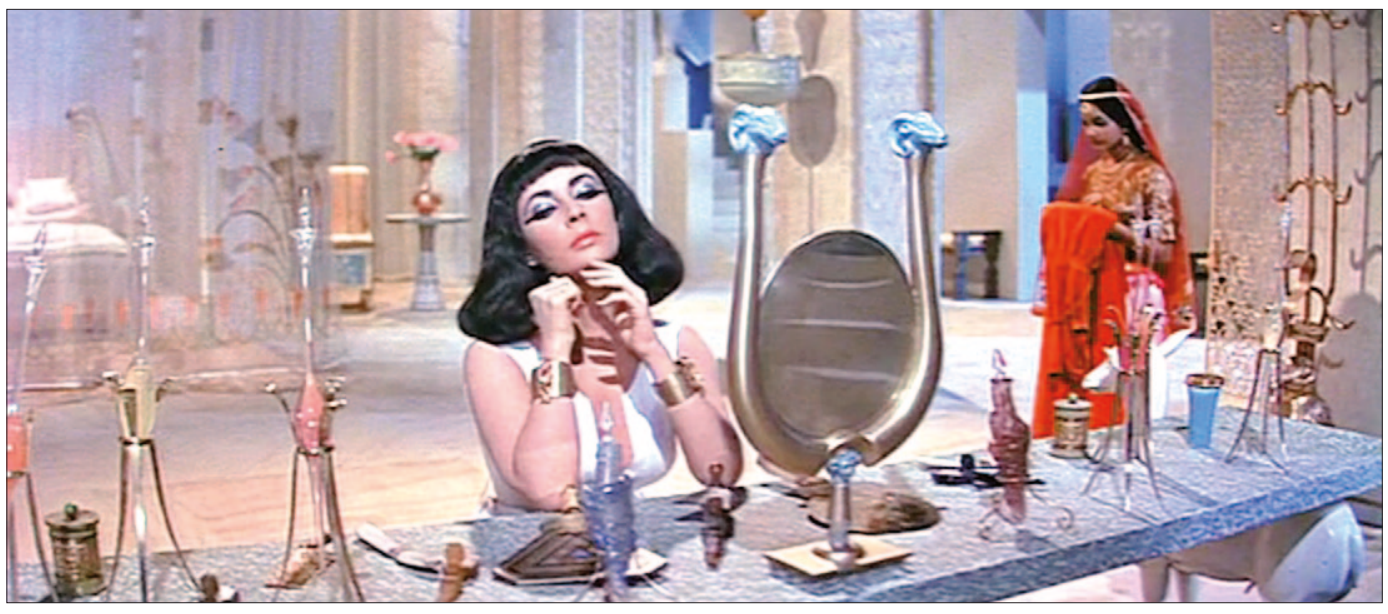

Abb. 3.3 - Einst. 38 nach SP 3, Cleopatra 


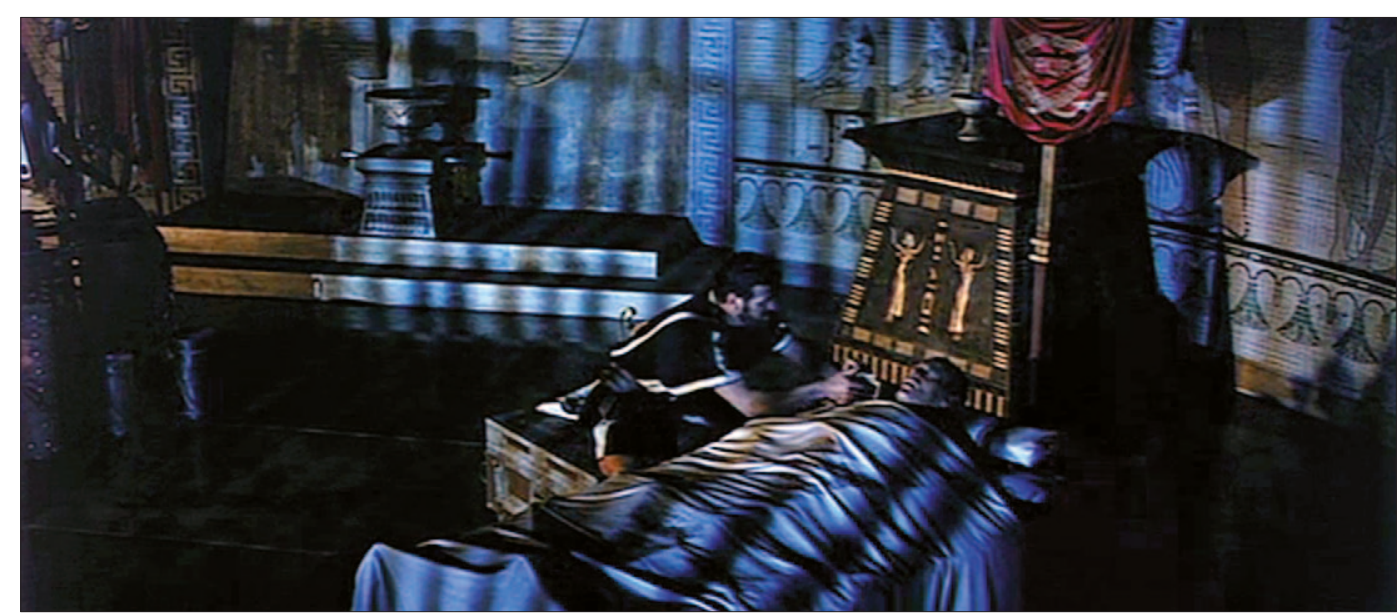

Abb. 3.4 - Einst. 39 nach SP 3, Cleopatra

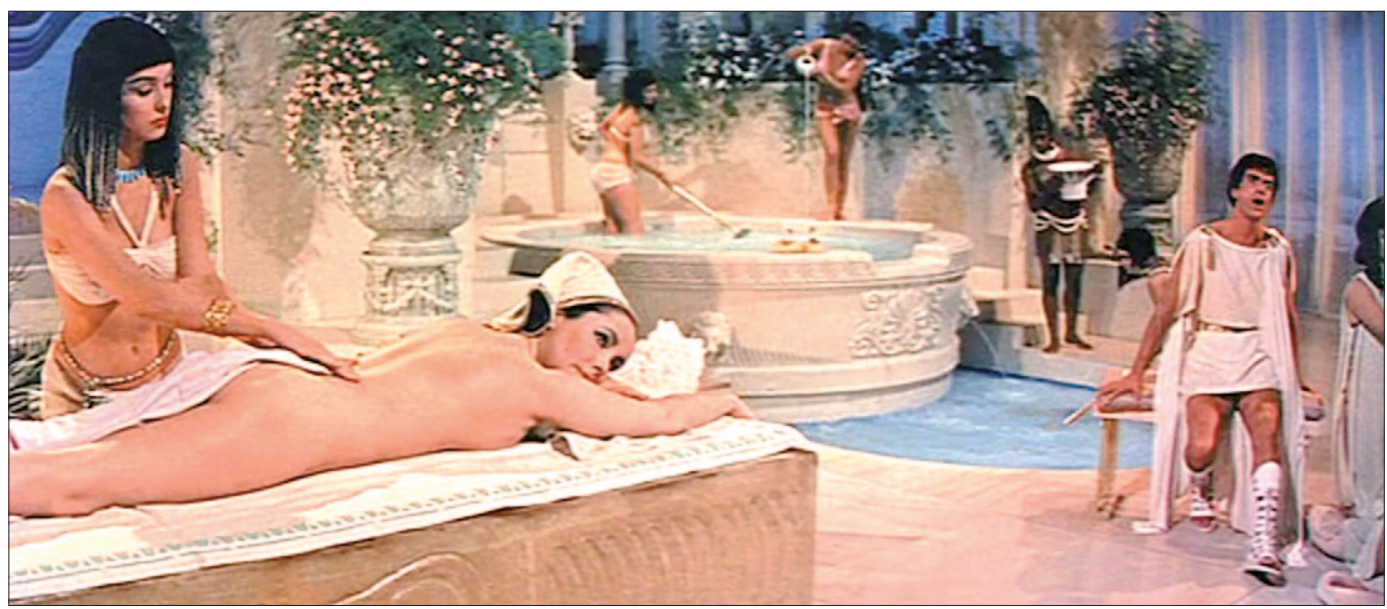

Abb. 3.5 - Einst. 40 nach SP 3, Cleopatra

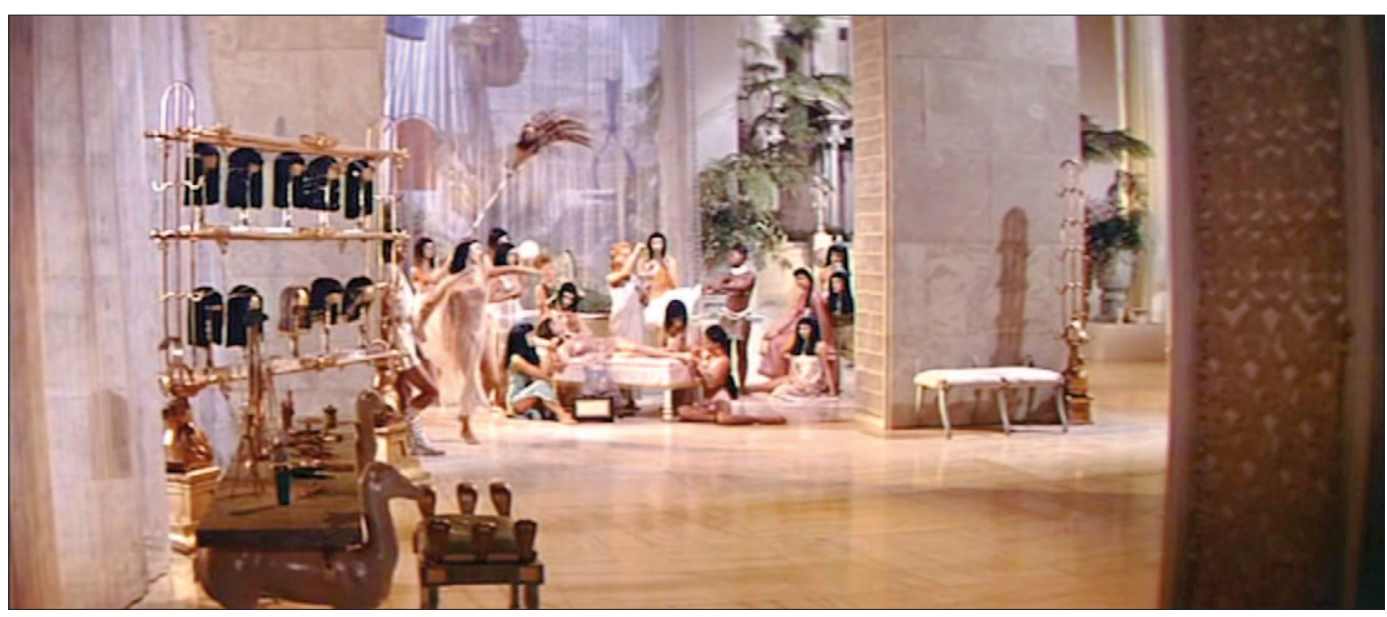

Abb. 3.6 - Einst. 53 nach SP 3, Cleopatra 


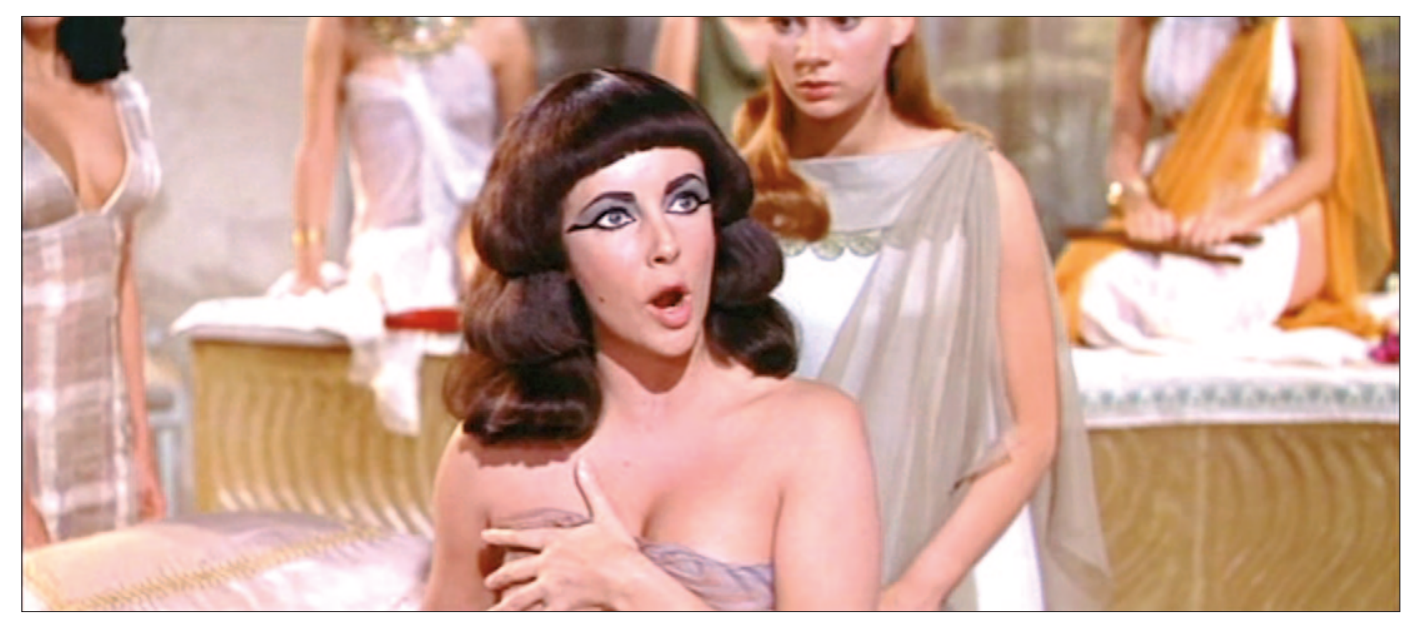

Abb. 3.7 - Einst. 60 nach SP 3, Cleopatra
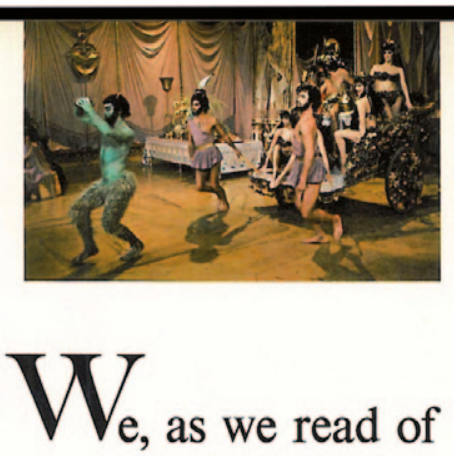

the deeds of the Queen of Egypt, must doff our modern conception of right and wrong; and, as we pace the courts of the Ptolemies, and breathe the atmosphere of the first -0 century before Christ, we must not commit the anachronism of criticizing our surroundings from the standard of twenty centuries after Christ.

ARTHUR WEIGALL, HISTORIAN

Abb. 3.8 - 20th Century-Fox Corporation (Hg.), 1963, Cleopatra. New York, S. [5]. 


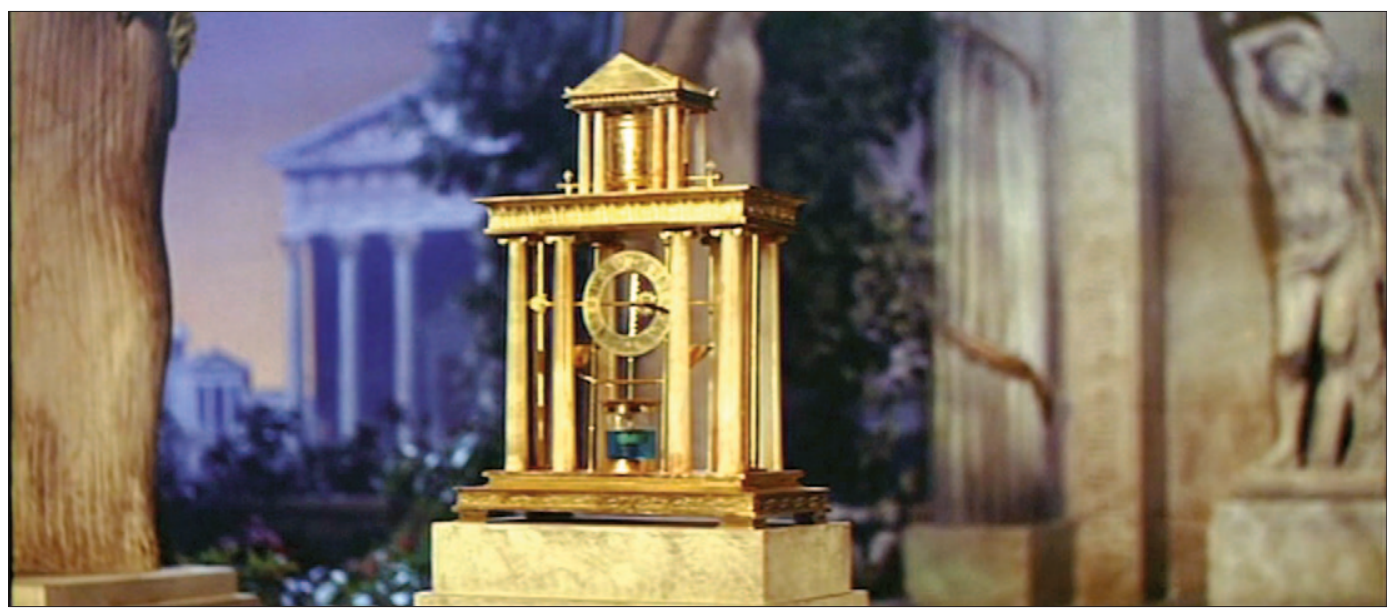

Abb. 3.9 - Mechanische Uhr im Haus Mark Antonys, Cleopatra

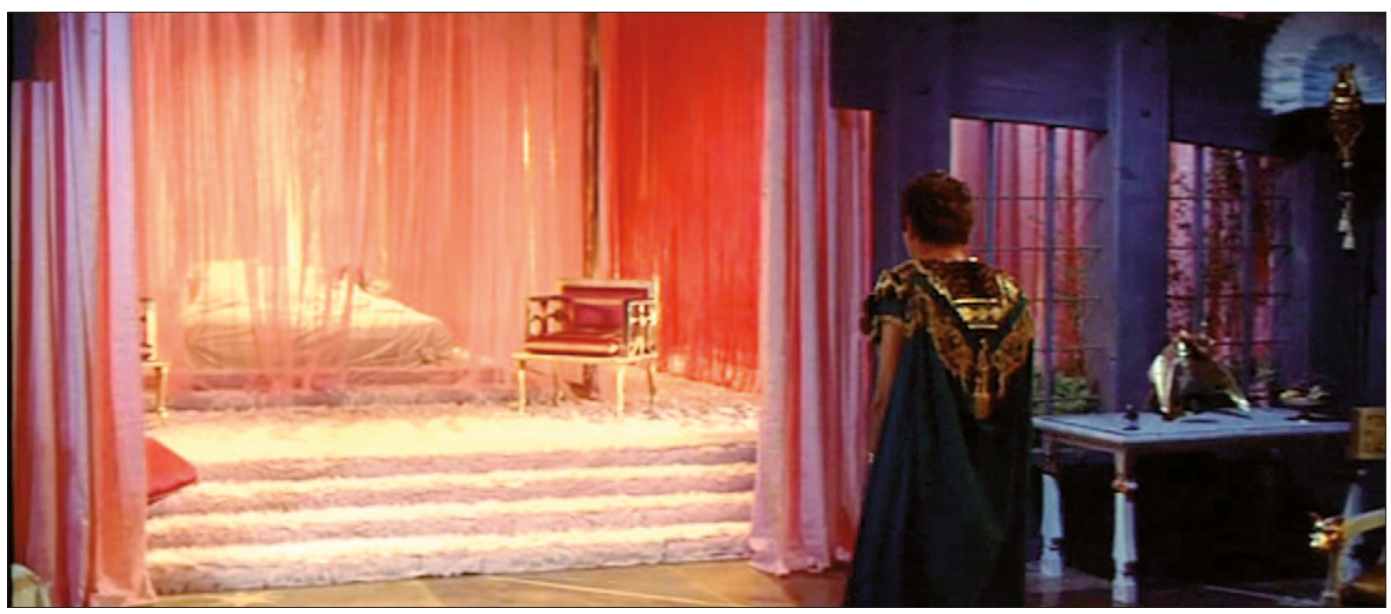

Abb. 3.10 - Bett auf Cleopatras goldener Barke, CLeopatra

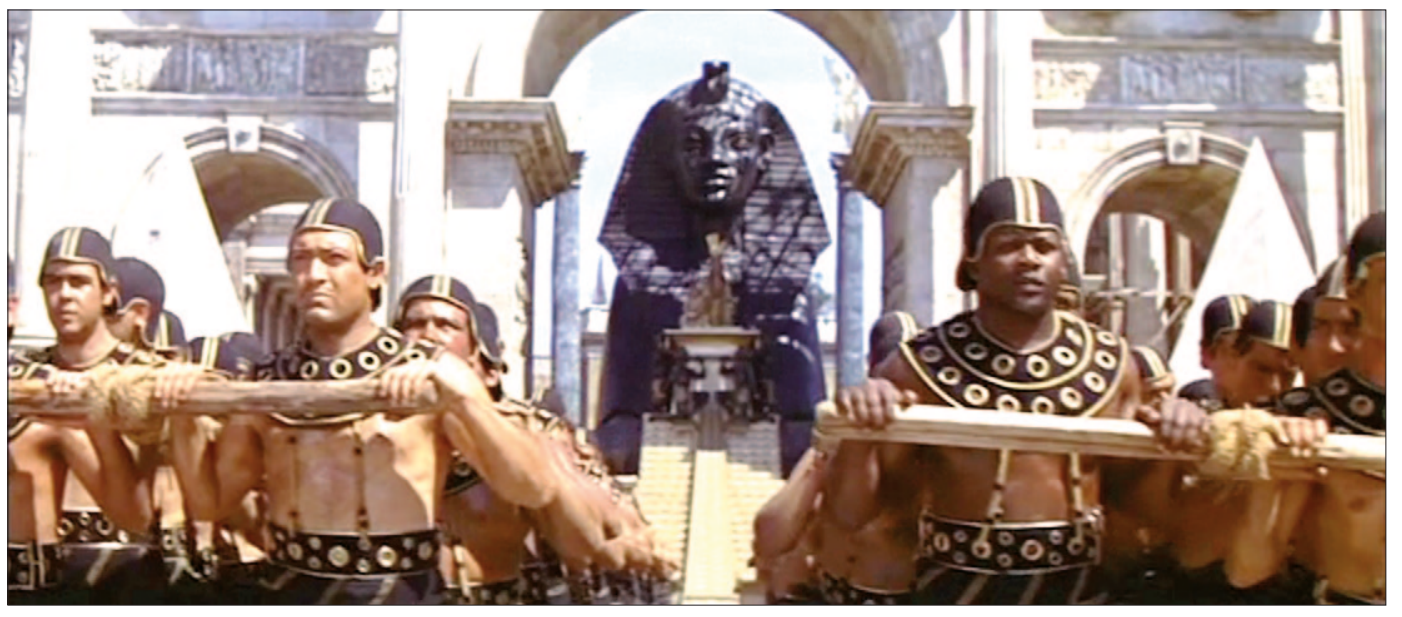

Abb. 3.11 - Schatten vom Filmkulissengerüst auf Cleopatras Sphinx, CLEOPATRA 


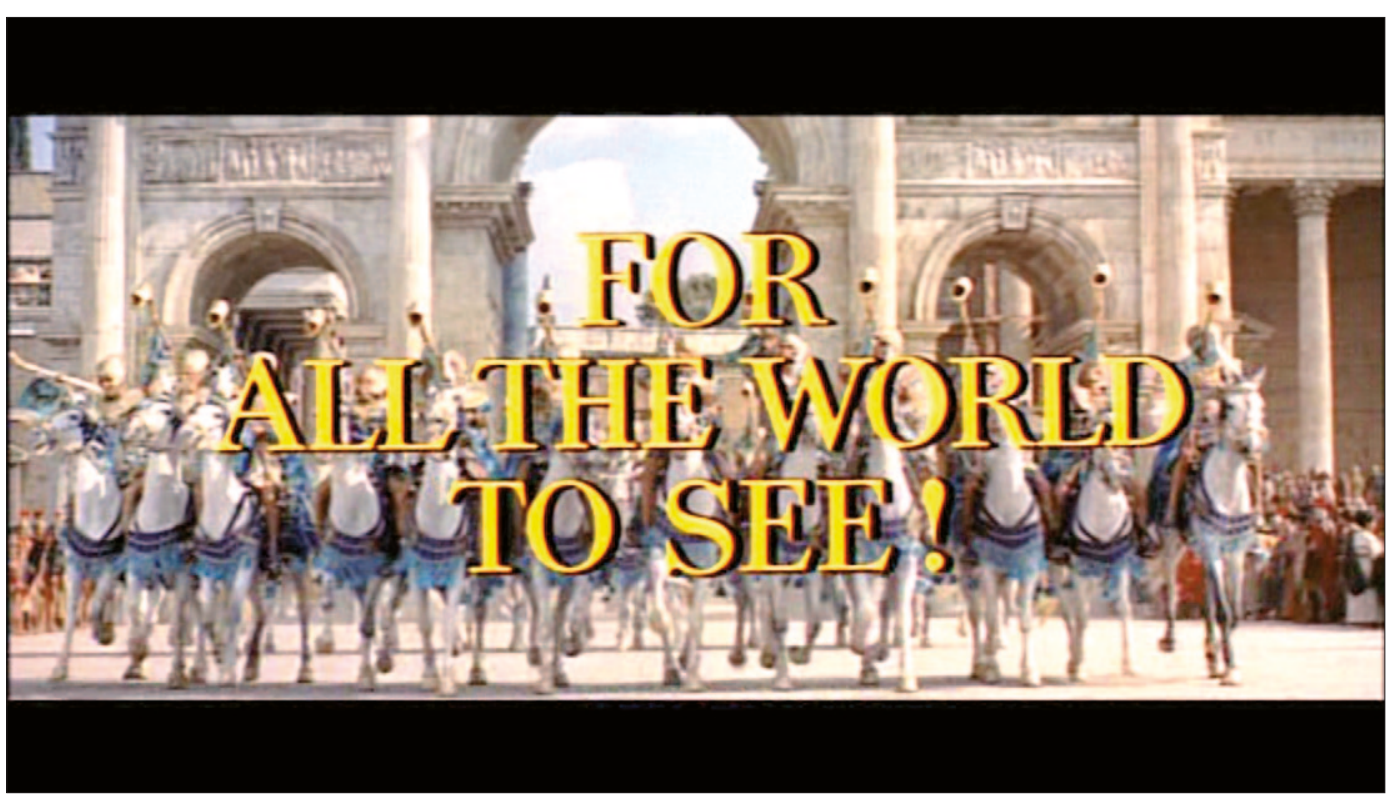

Abb. 3.12 - Zeitgenössischer Trailer zu CLeOPATRA

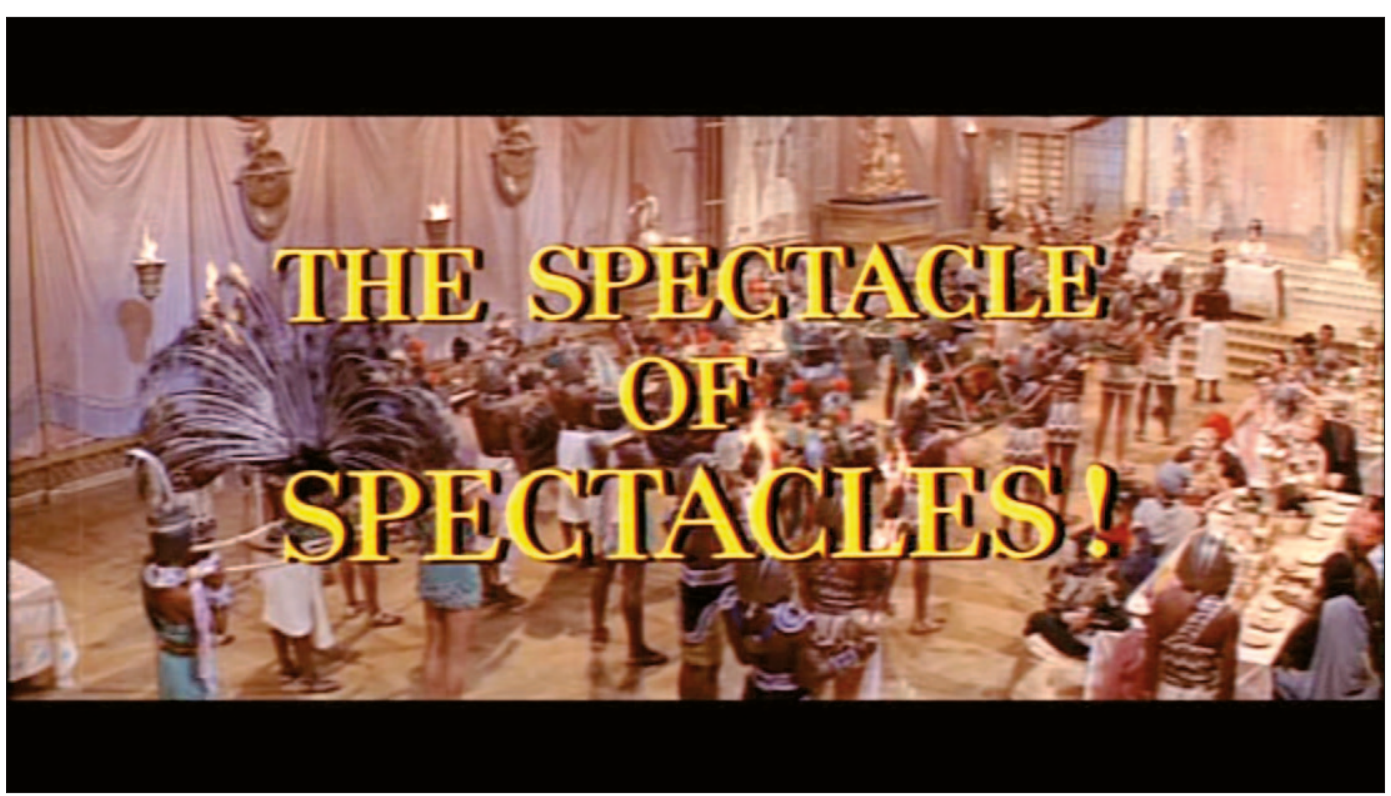

Abb. 3.13 - Zeitgenössischer Trailer zu CLEOPATRA 


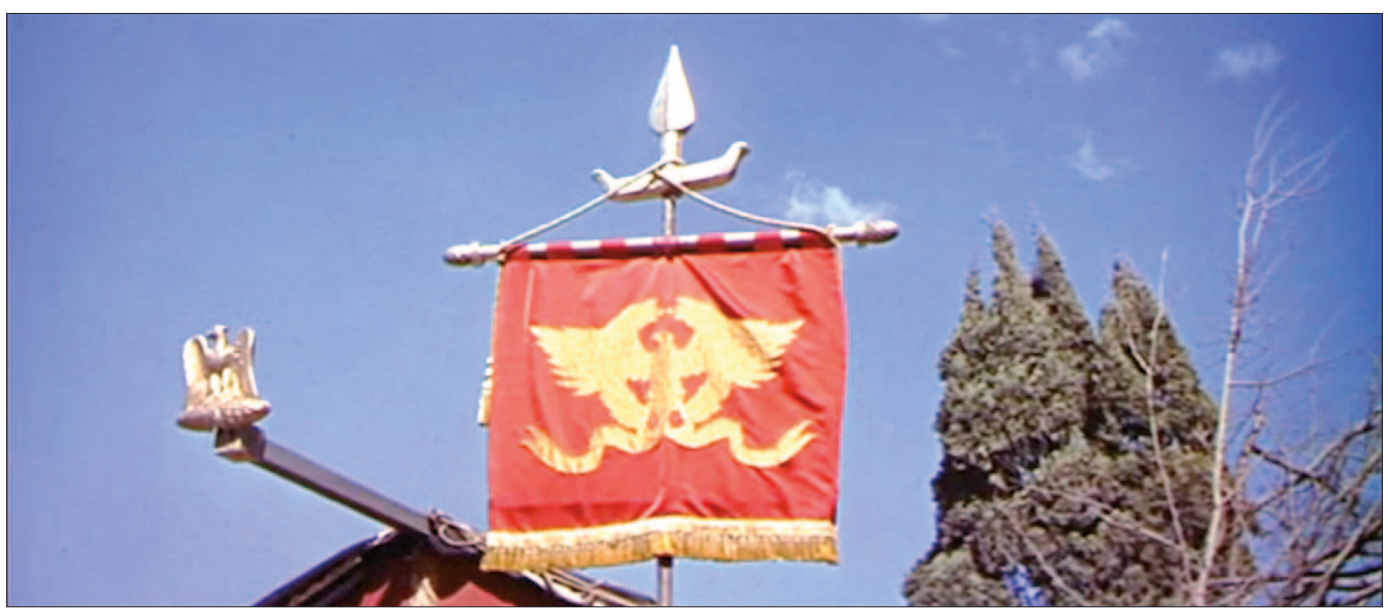

Abb. 3.14 - Fotografisches Bild, CLEOPATRA

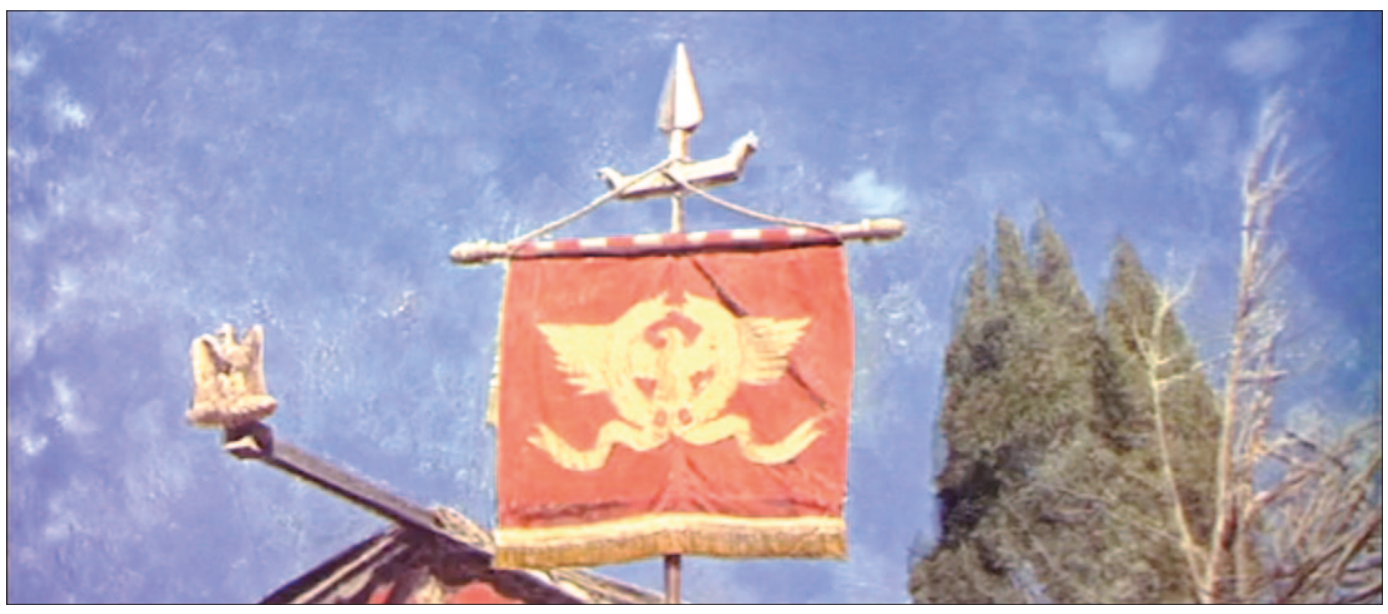

Abb. 3.15 - Gemaltes Bild, Cleopatra

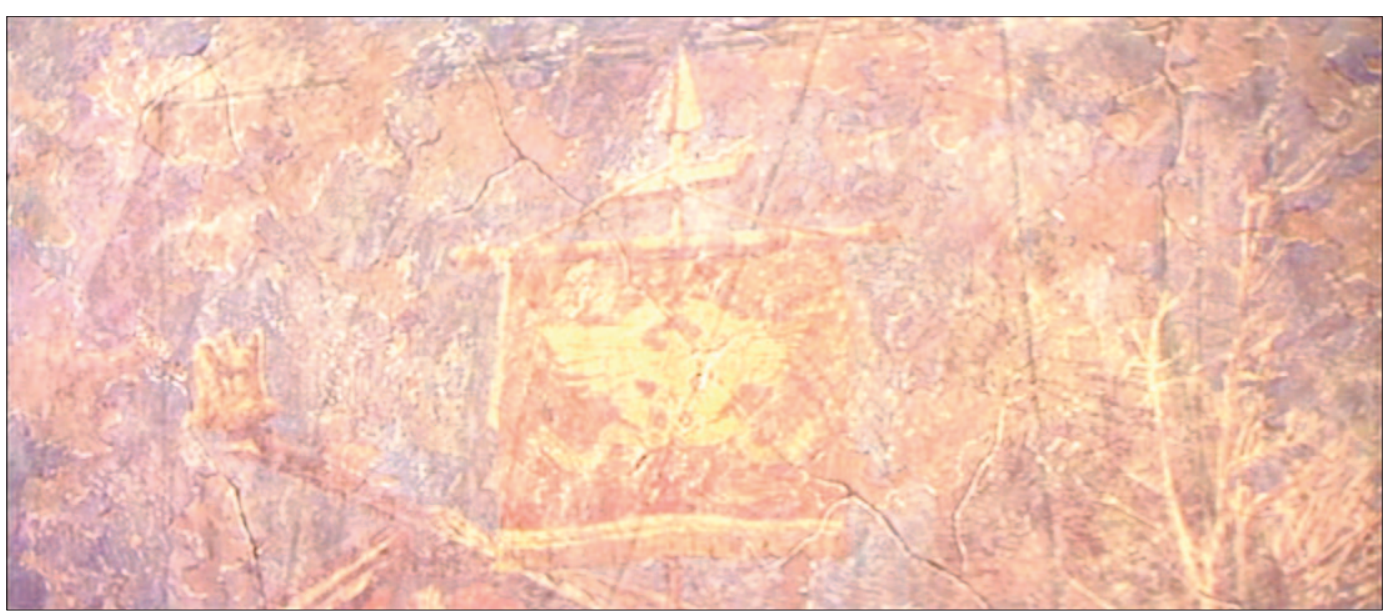

Abb. 3.16 - Beschädigtes Fresko, CLEOPATRA 


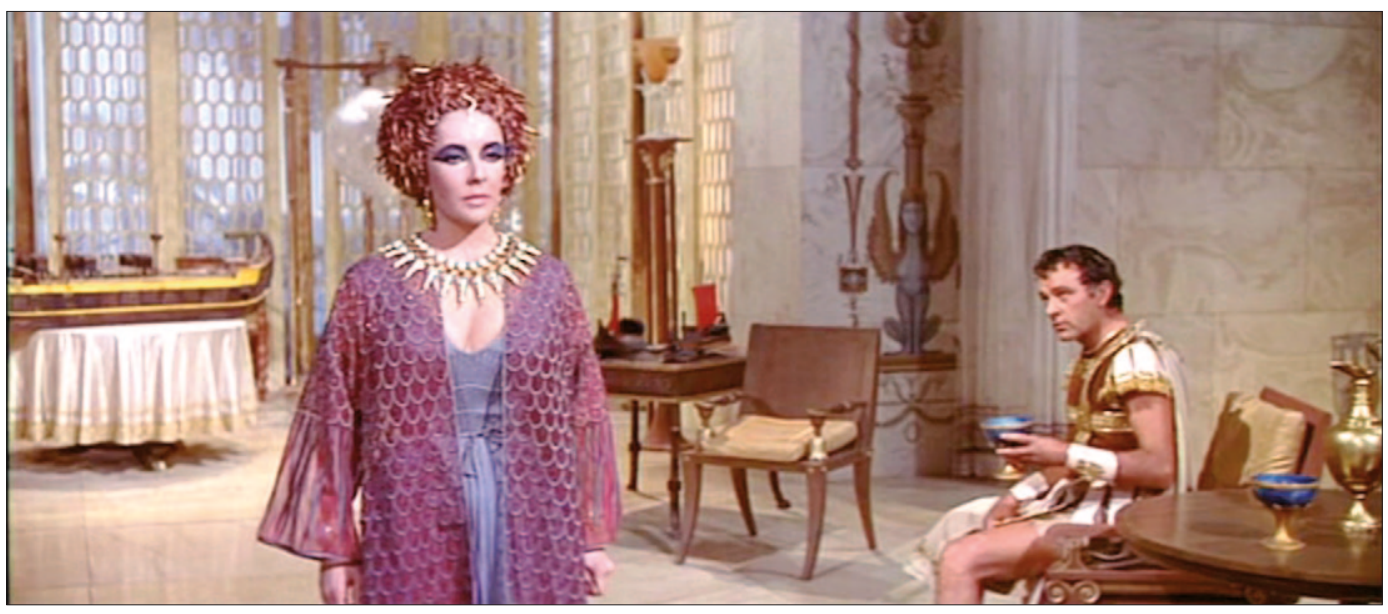

Abb. 3.17 - Schiffsmodelle in Cleopatras Palast in Alexandria, Cleopatra

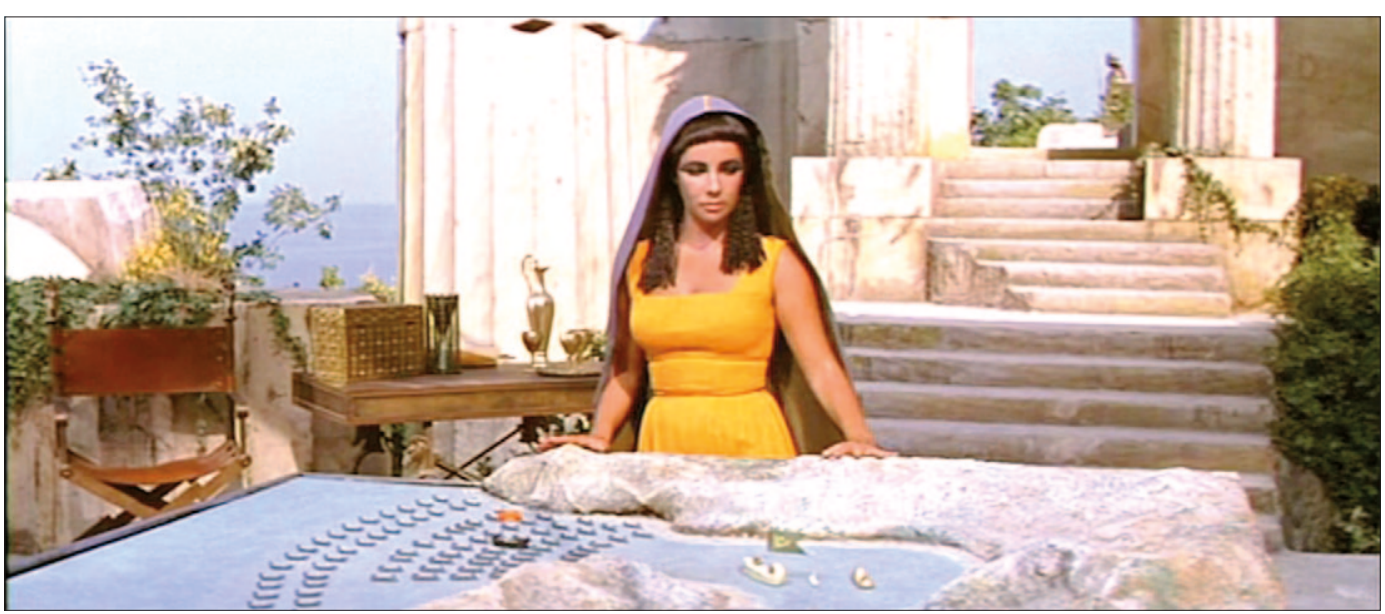

Abb. 3.18-Cleopatra am Modell der Bucht von Actium, Cleopatra

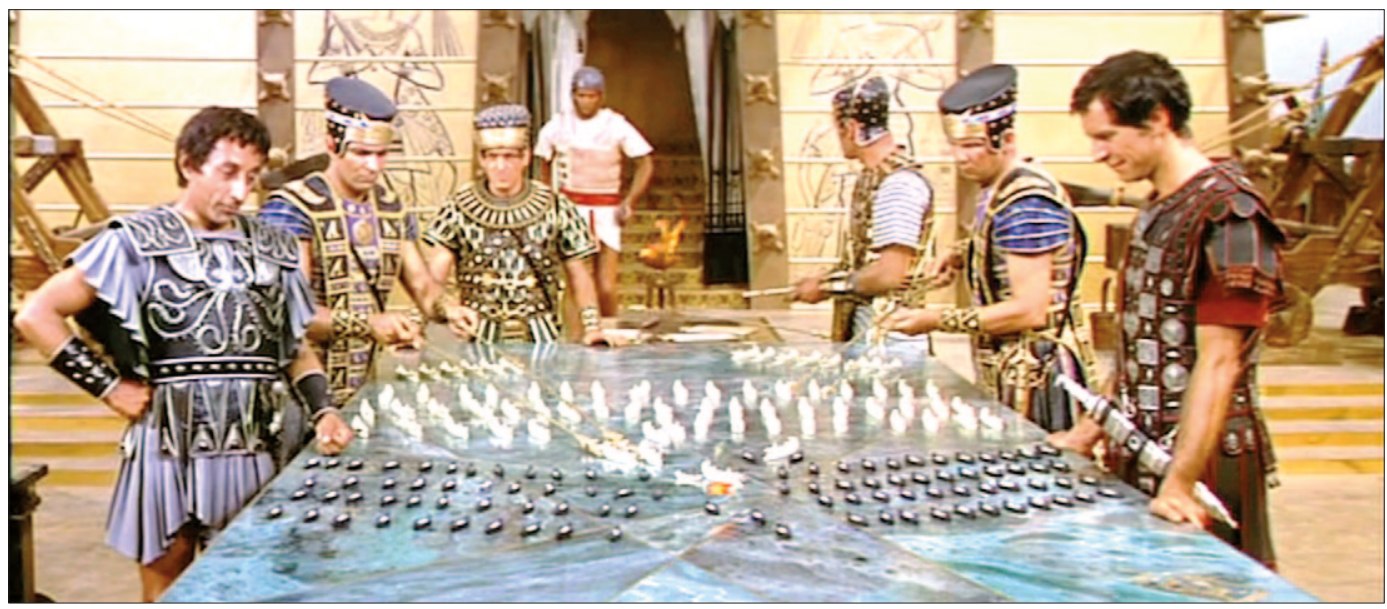

Abb. 3.19 - Modell der Schlacht von Actium auf Cleopatras Schiff, Cleopatra 


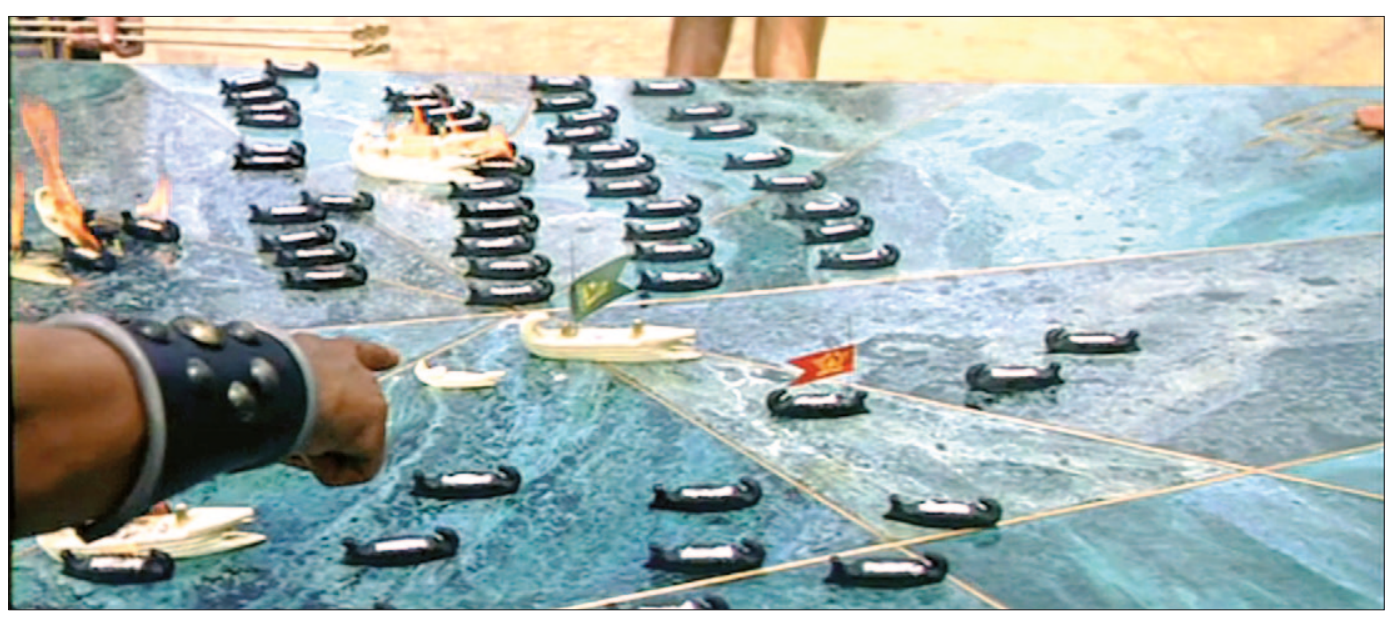

Abb. 3.20 - Modell der Schlacht von Actium auf Cleopatras Schiff, CleOPATRA

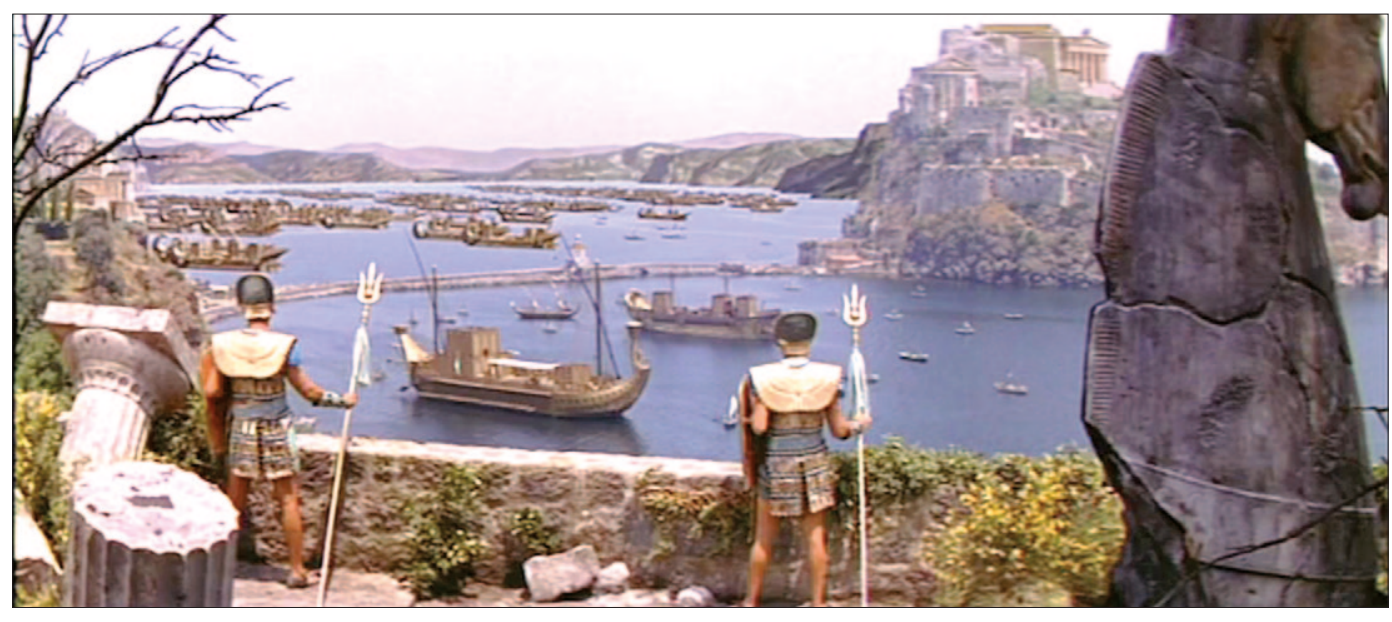

Abb. 3.21 - Die Bucht von Actium mit echten Schiffen und Matte Paintings, Cleopatra

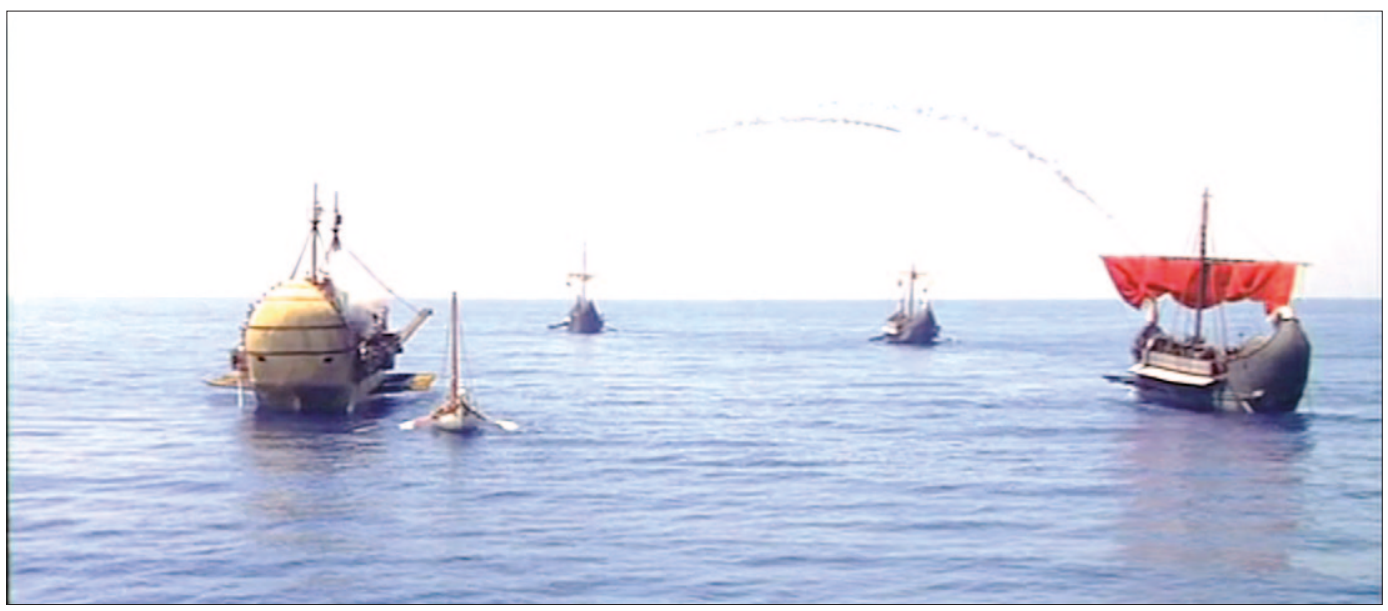

Abb. 3.22 - Die fünf realen Schiffe des Drehs, CLEOPATRA 


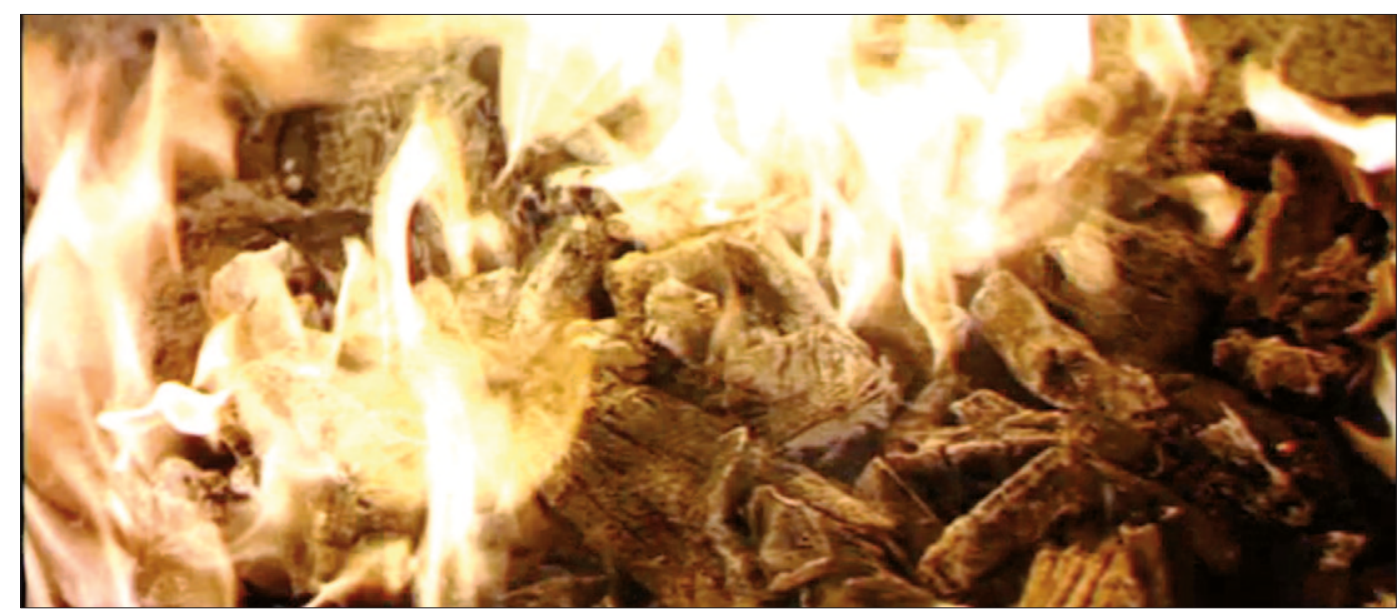

Abb. 3.23 - POV auf die Kohlenschale, Cleopatra

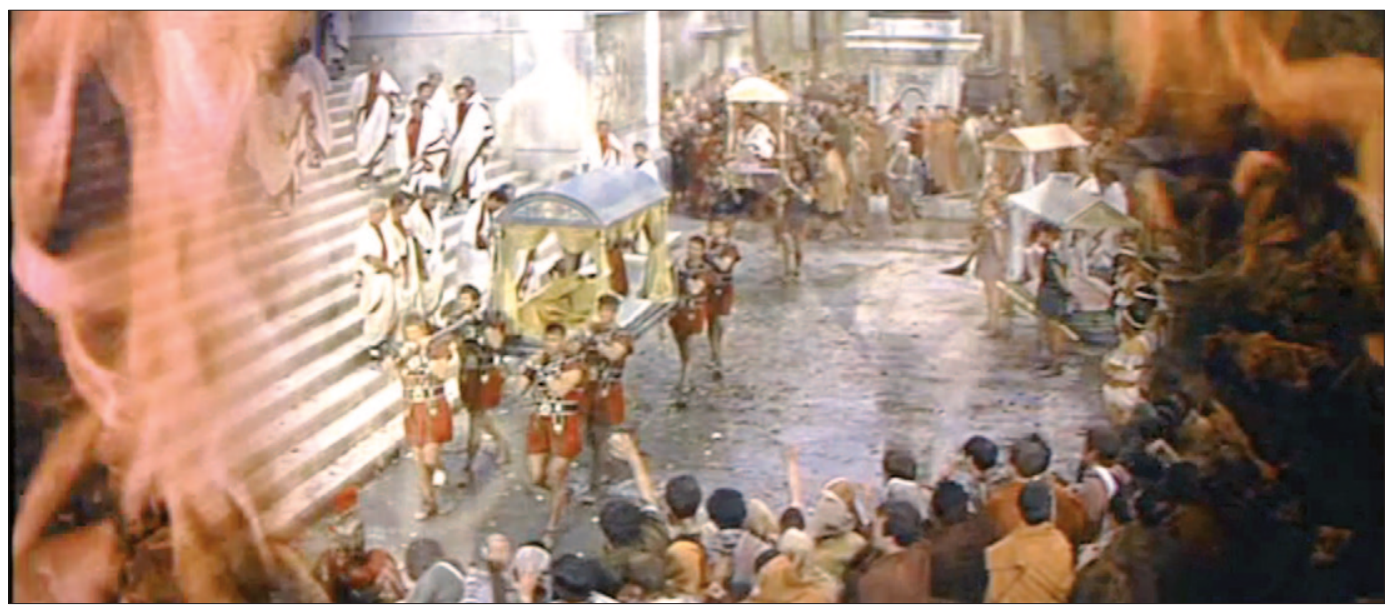

Abb. 3.24 - POV mit Bildern vom Forum, CLeOPATRA

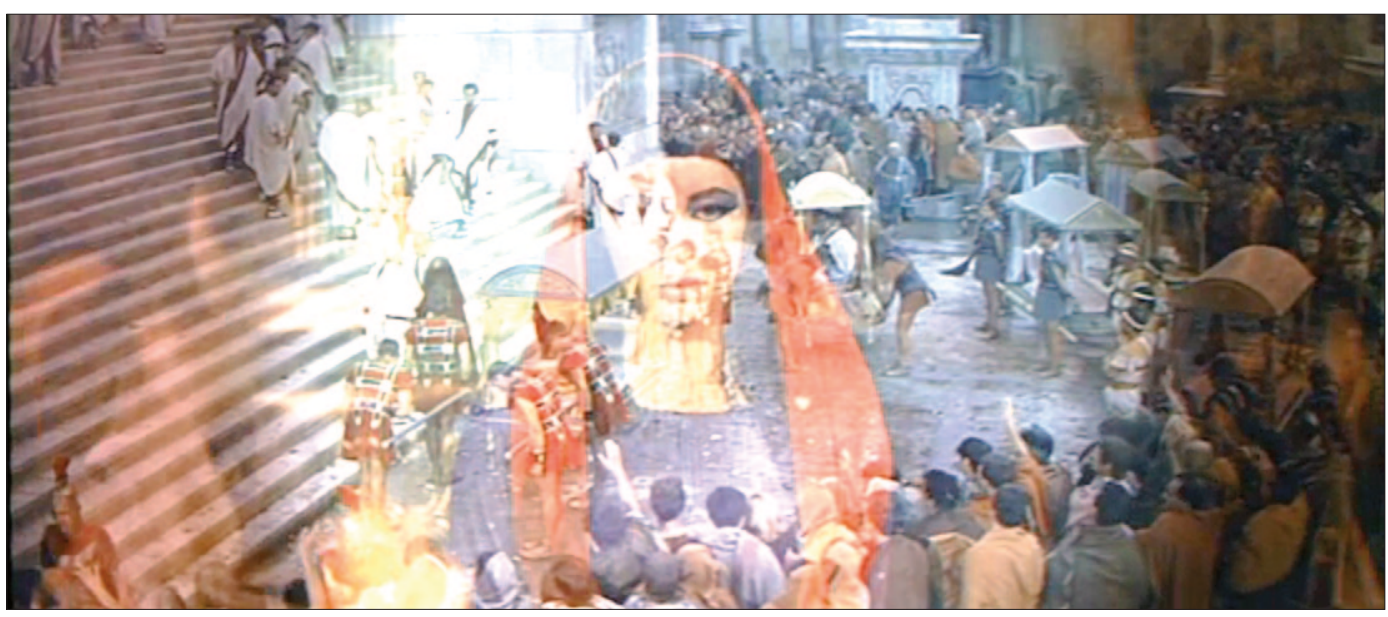

Abb. 3.25 - POV mit Bildern vom Forum und Cleopatra, CLEOPATRA 


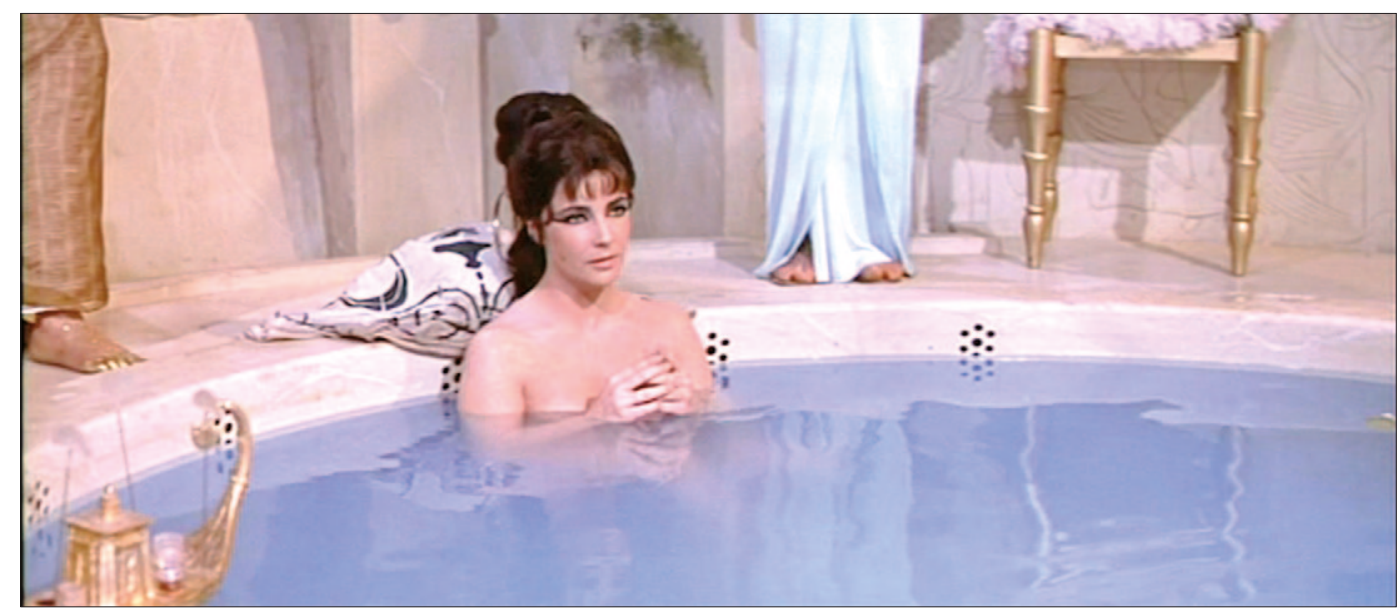

Abb. 3.26 - Cleopatra in ihrem Bad, Cleopatra

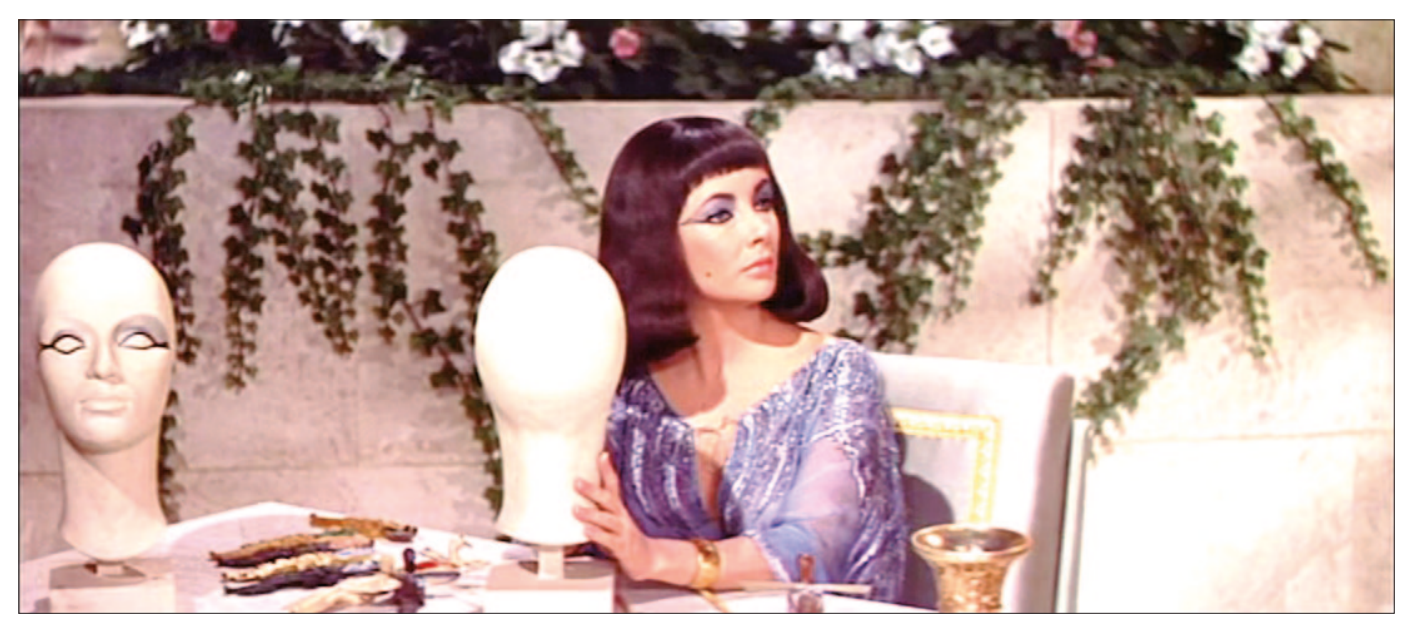

Abb. 3.27 - Cleoaptra erprobt Make-up-Varianten, CLEOPATRA

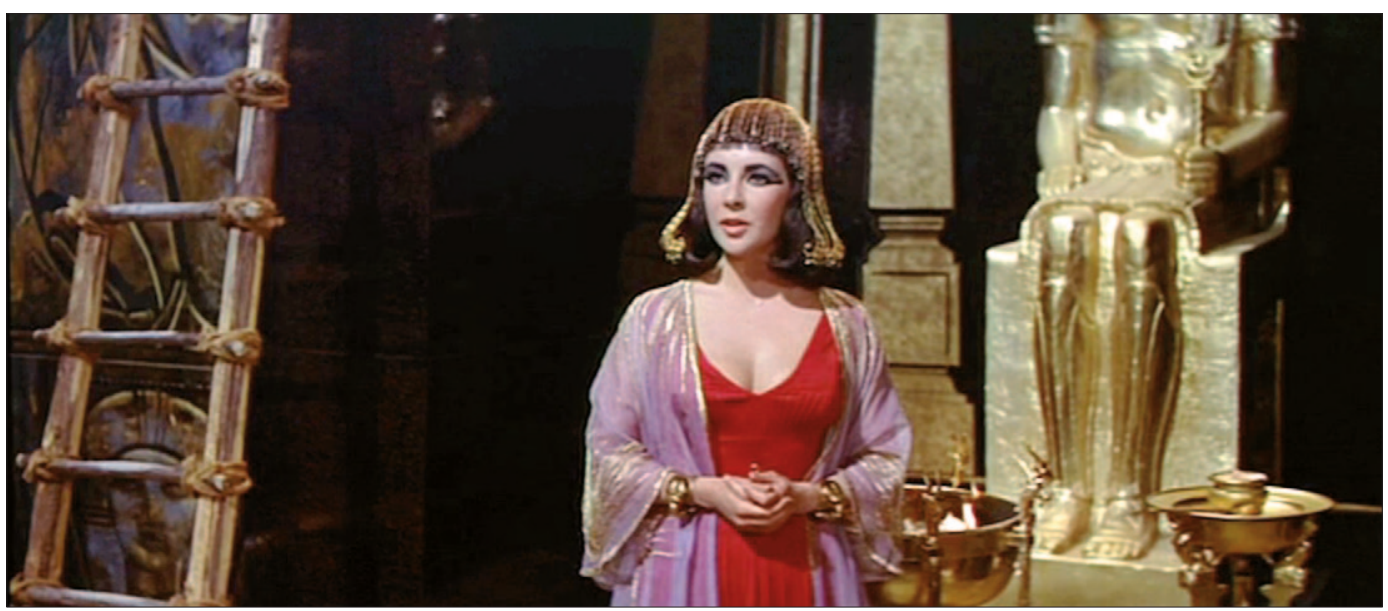

Abb. 3.28 - Cleopatra bei den Bauarbeiten an ihrem Mausoleum, Cleopatra 


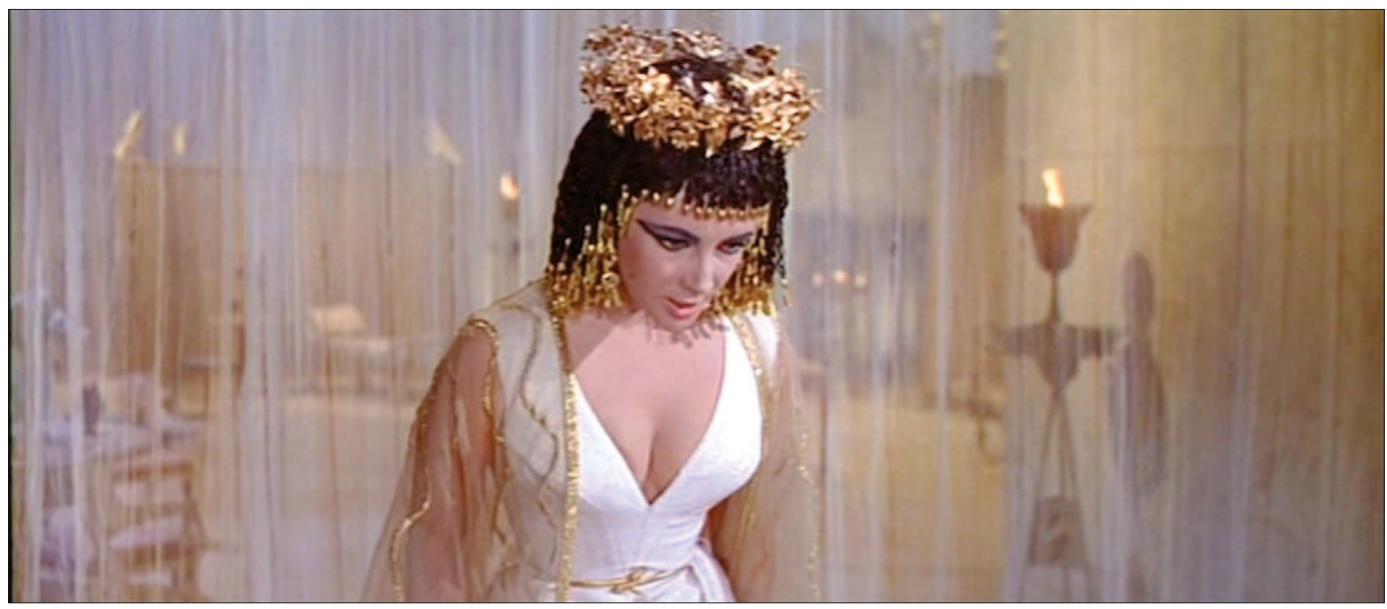

Abb. 3.29 - Cleopatra im Streit mit Mark Antony, CleopATRA

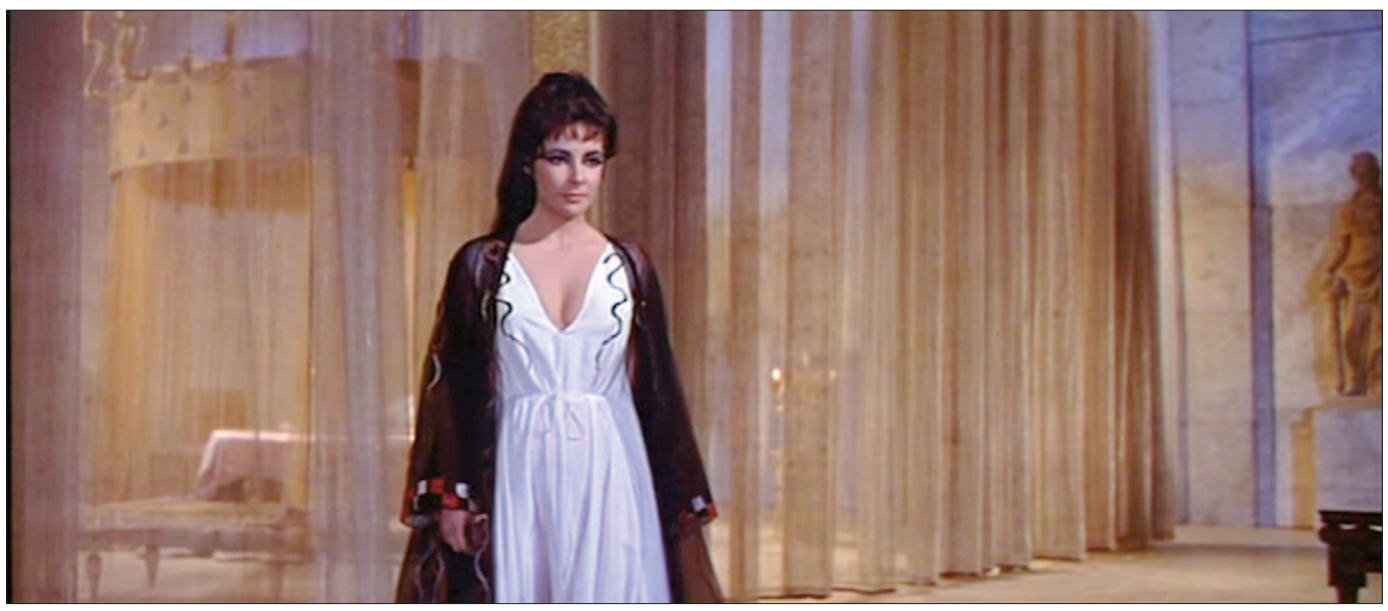

Abb. $3 \cdot 30$ - Cleopatra im Streit mit Mark Antony, CLEOPATRA

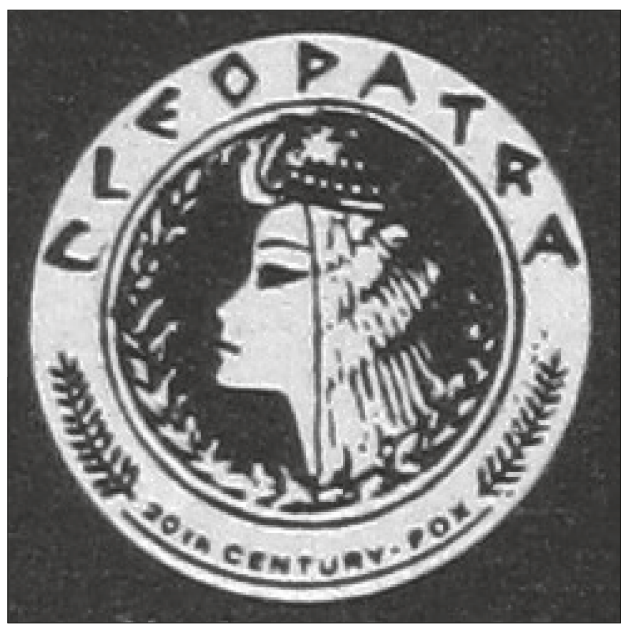

Abb. 3.31 - „Cleopatra-Siegel« der 2oth Century-Fox, VOGUE, Vol. 142, Nr. 3, (1. Aug. 1963) 


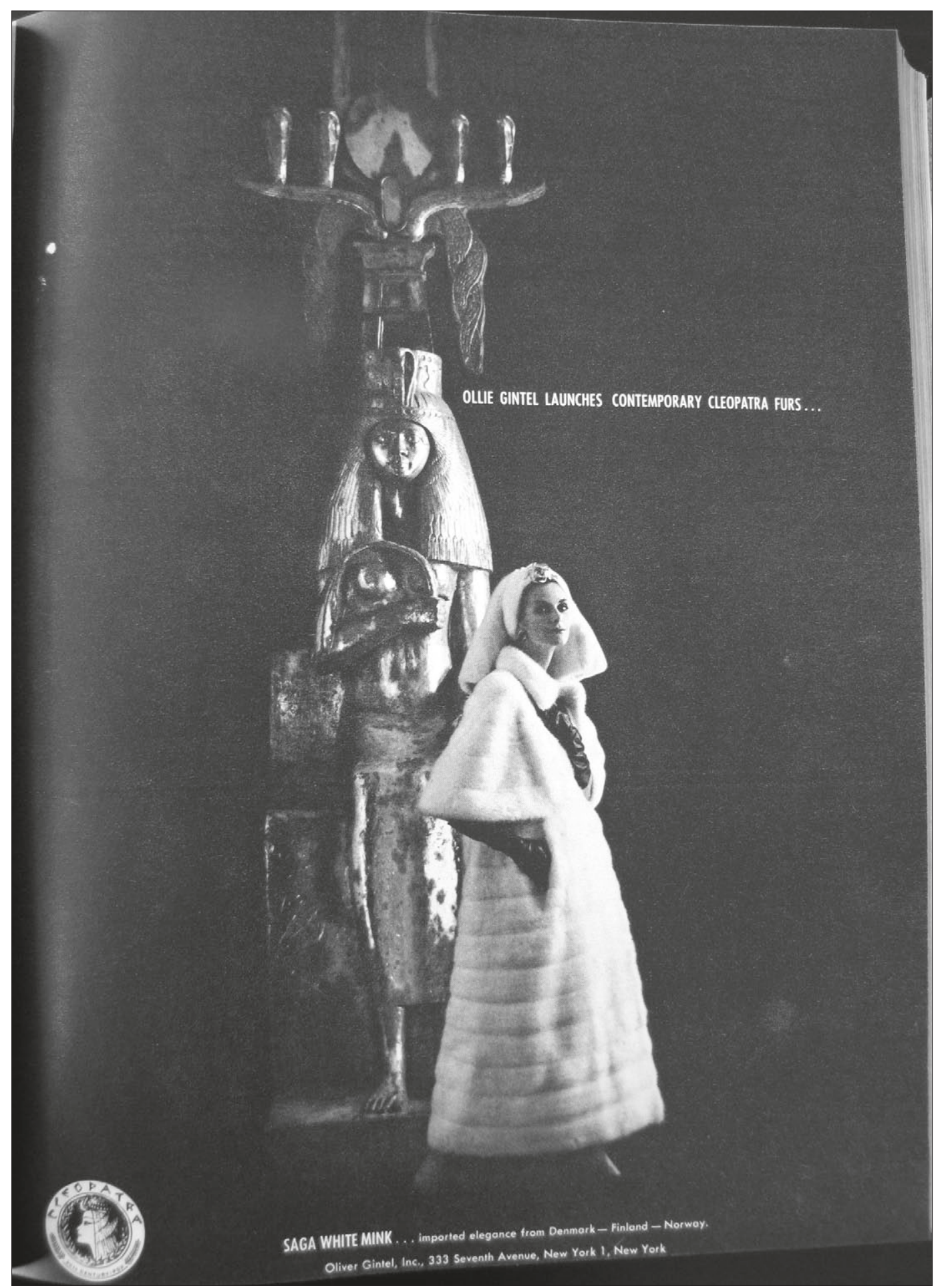

Abb. 3.32 - Anzeige für Oliver Gintel, VOGUE, Vol. 142, Nr. 3 


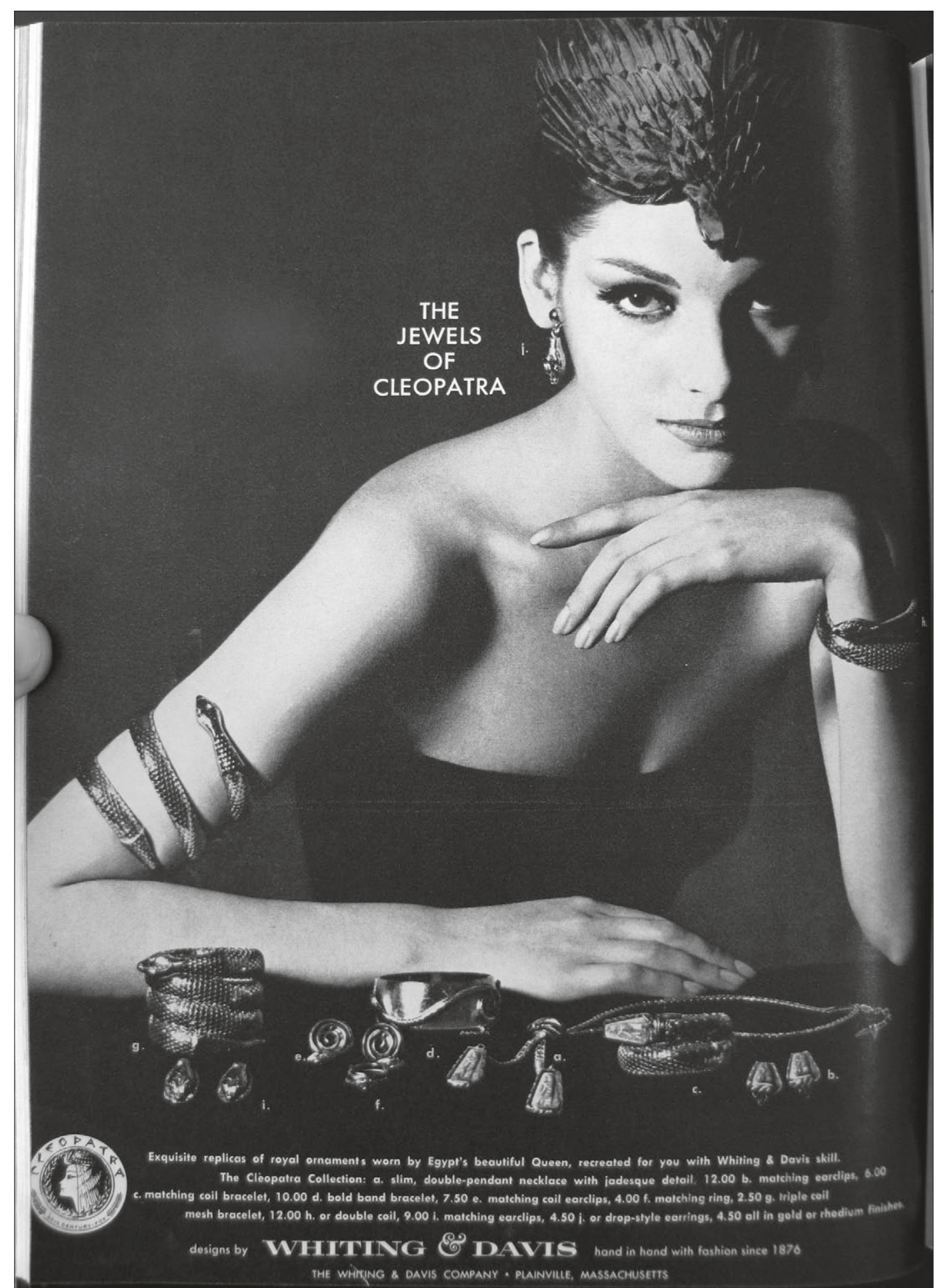

Abb. 3.33 - Anzeige für Whiting \& Davis, VOGUE, Vol. 142, Nr. 3 


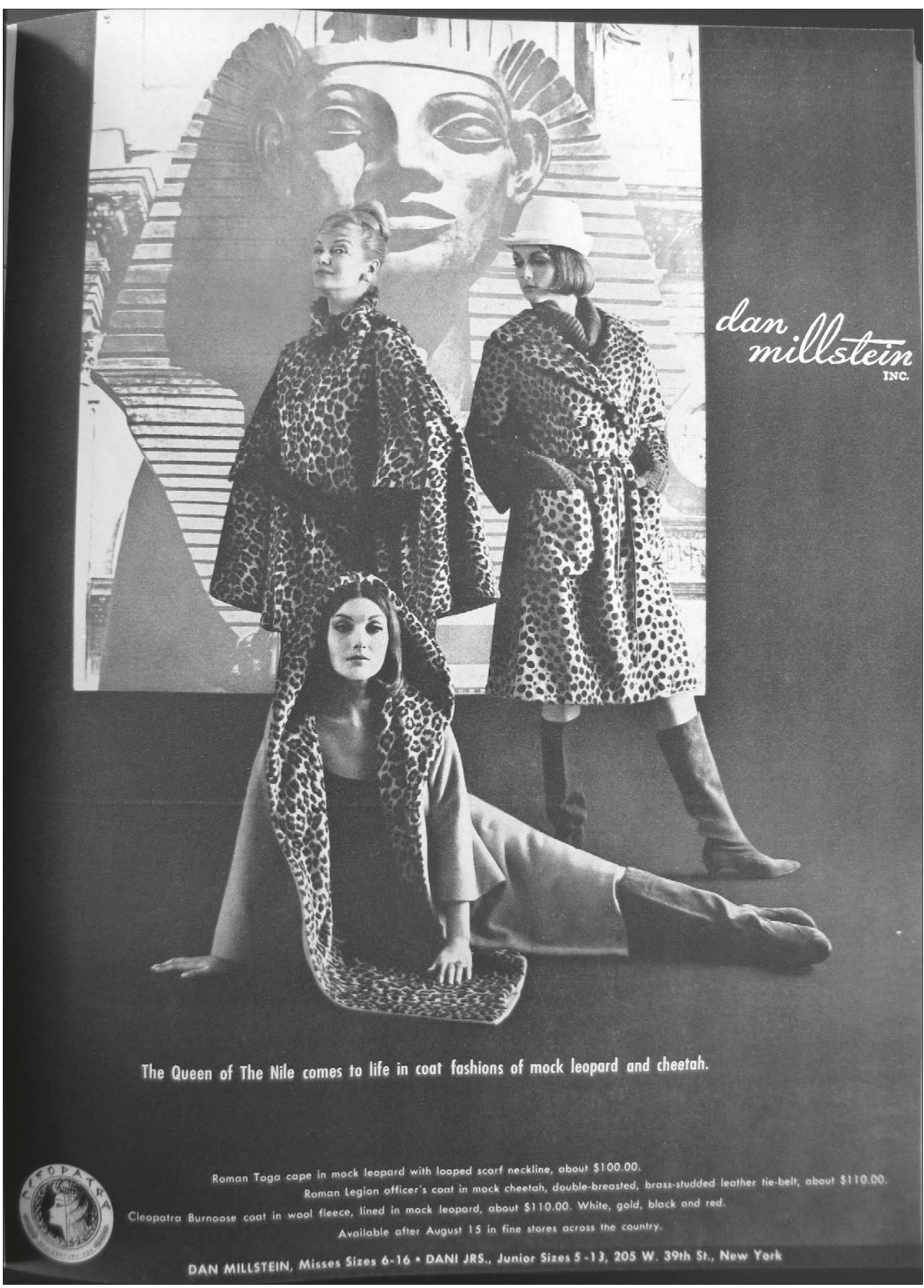

Abb. 3.34 - Anzeige für Dan Millstein Inc., VOGUE, Vol. 142, Nr. 3 


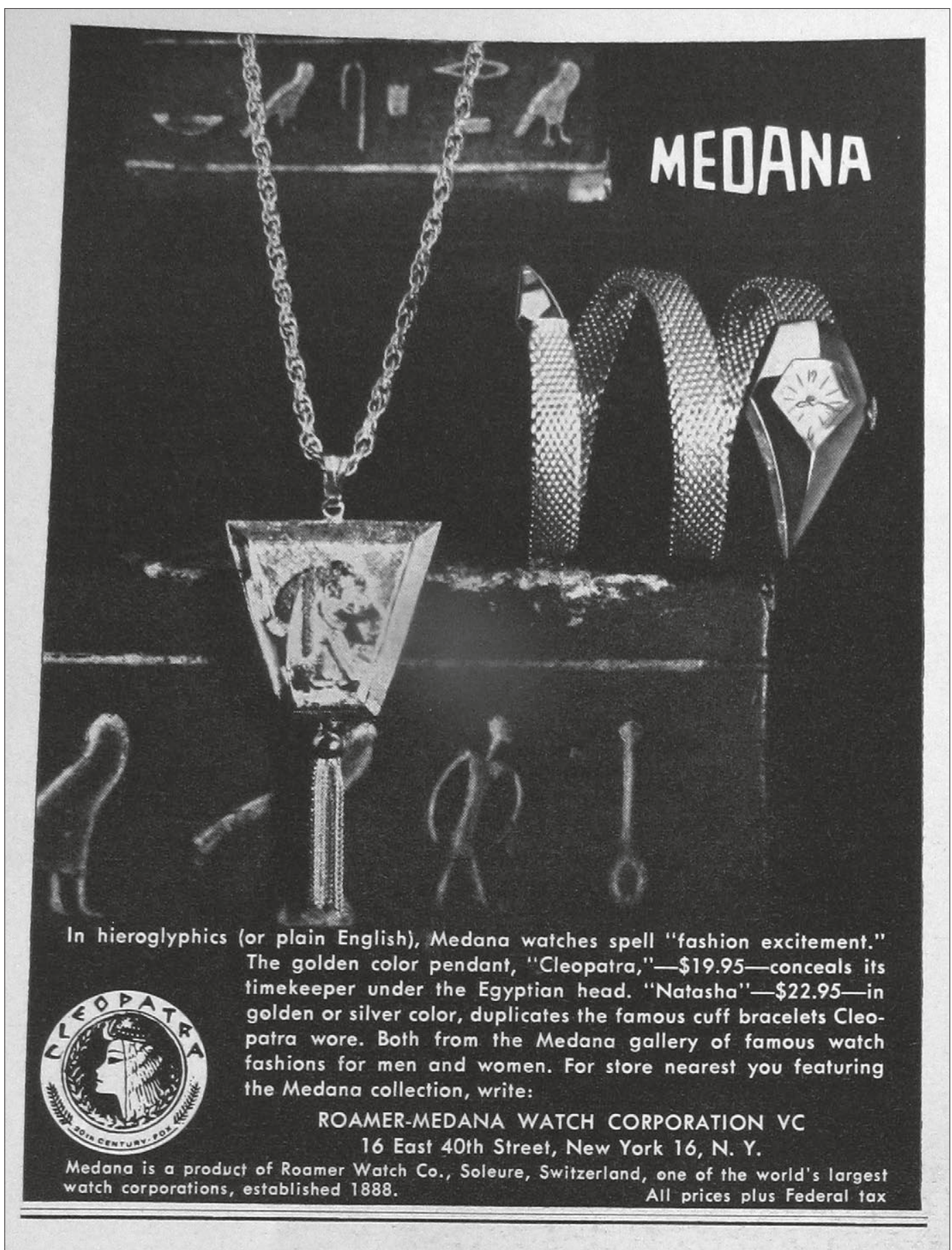

Abb. 3.35 - Anzeige für Roamer-Medana Watch Corporation, VOGUE, Vol. 142, NR. 3 


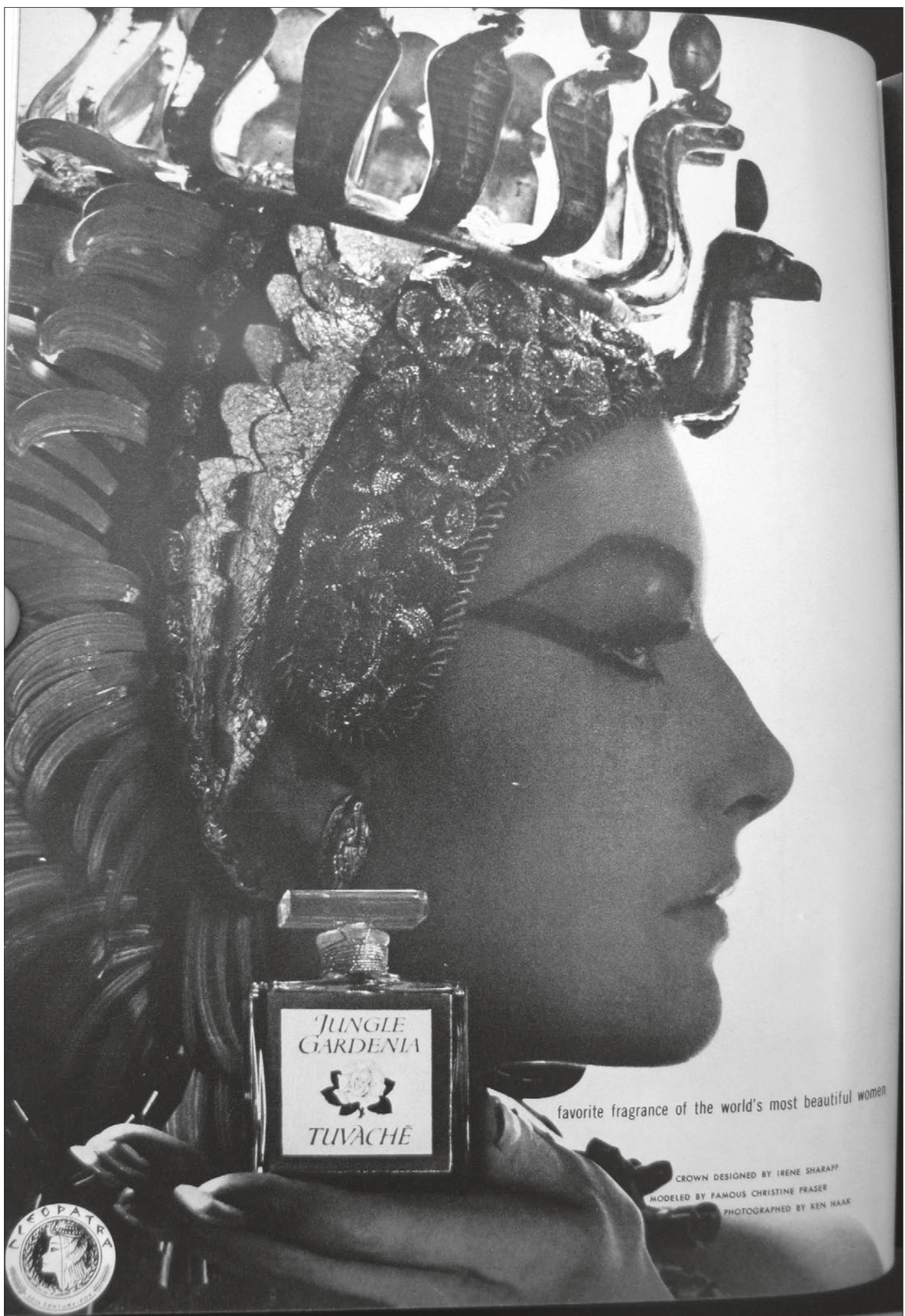

Abb. 3.36 - Anzeige für Tuvaché, VOGUE, Vol. 142, Nr. 3 


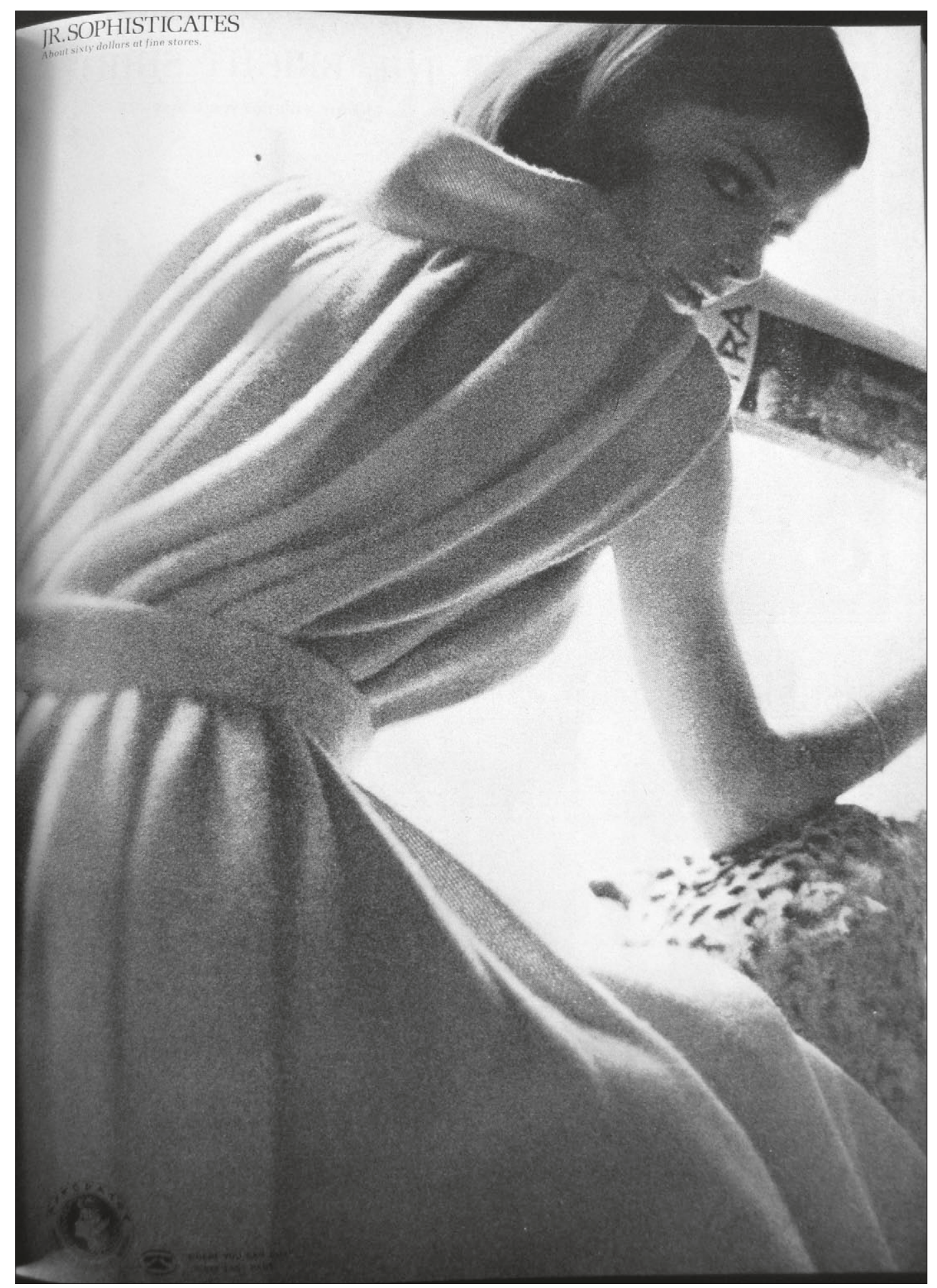

Abb. 3.37 - Anzeige für JR.Sophisticates, VOGUE, Vol. 142, Nr. 3 


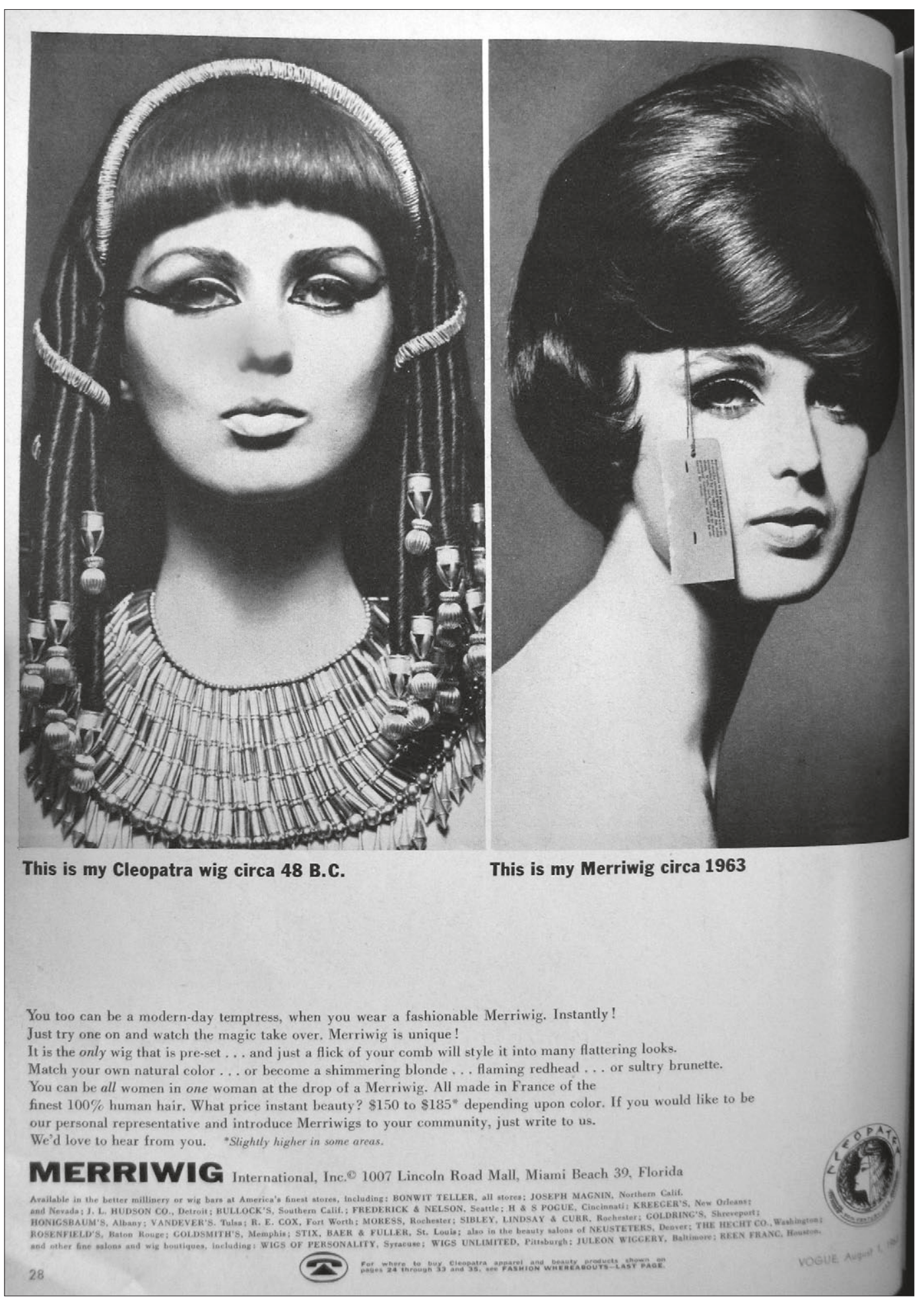

Abb. 3.38 - Anzeige für Merriwig International, VOGUE, Vol. 142, Nr. 3 


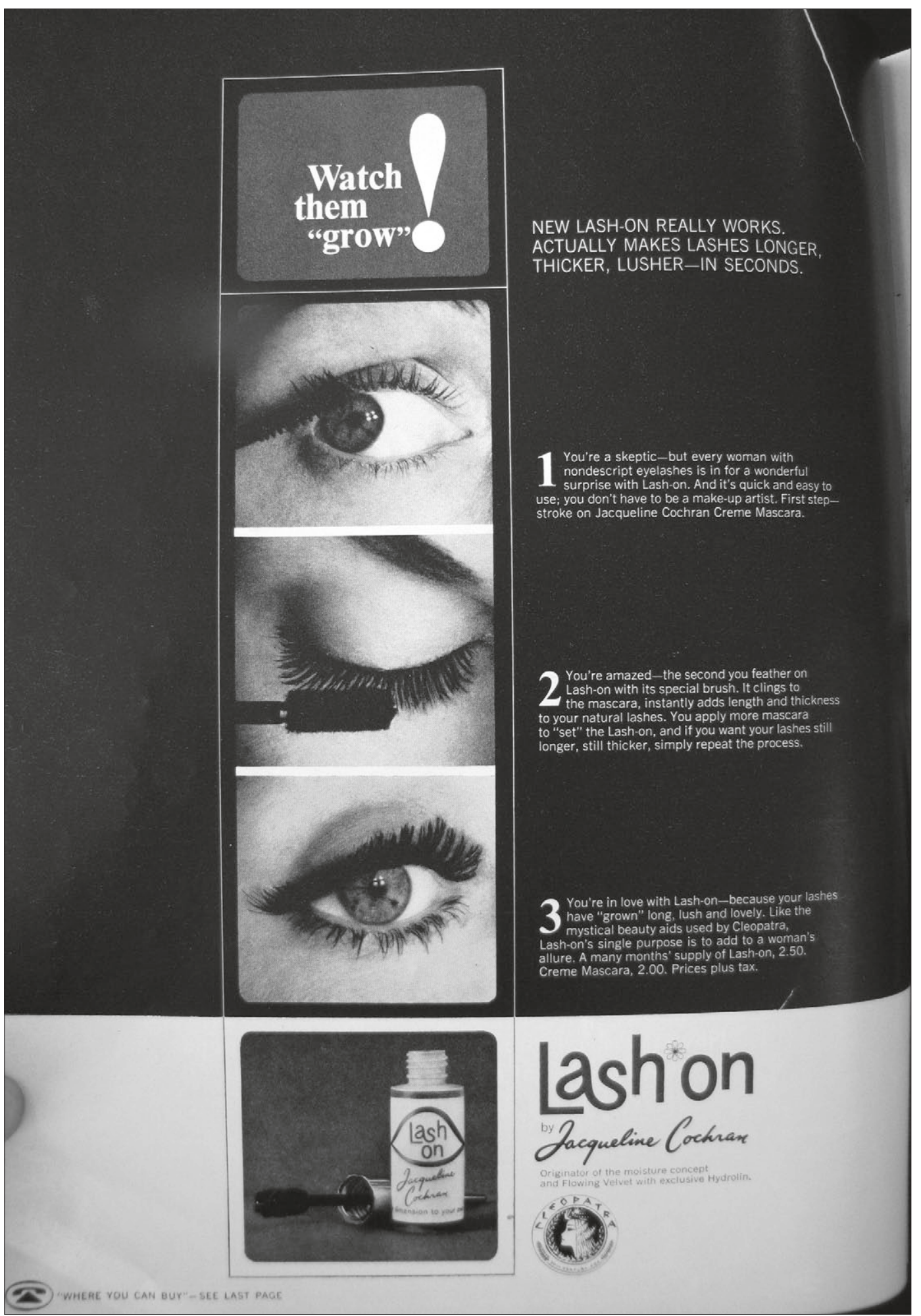

Abb. 3.39 - Anzeige für Jacqueline Cochran, VOGUE, Vol. 142, Nr. 3 


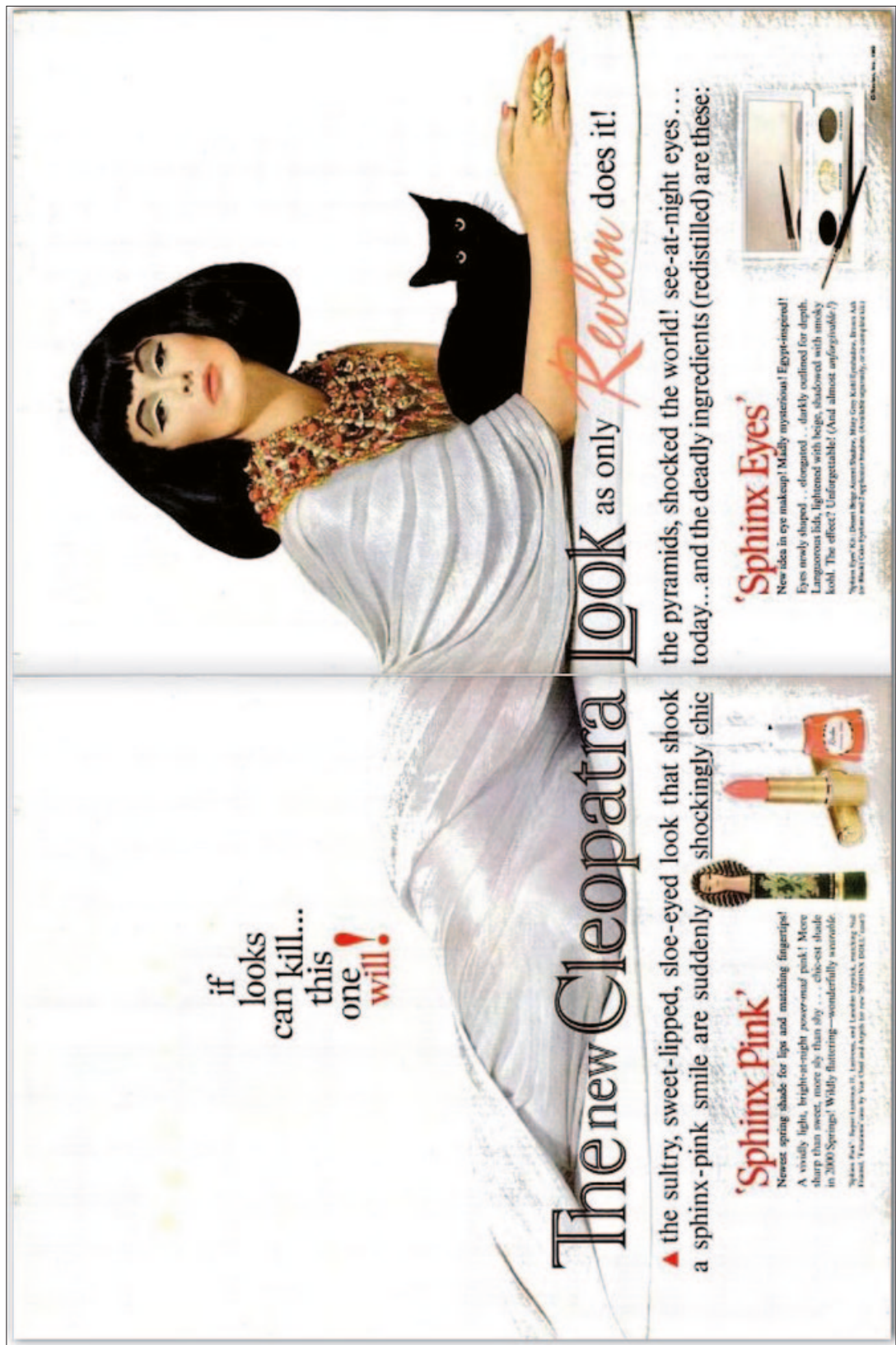

Abb. 3.40 - Anzeige für Revlon Sphinx Eye Makeup, 196oer JAHRE

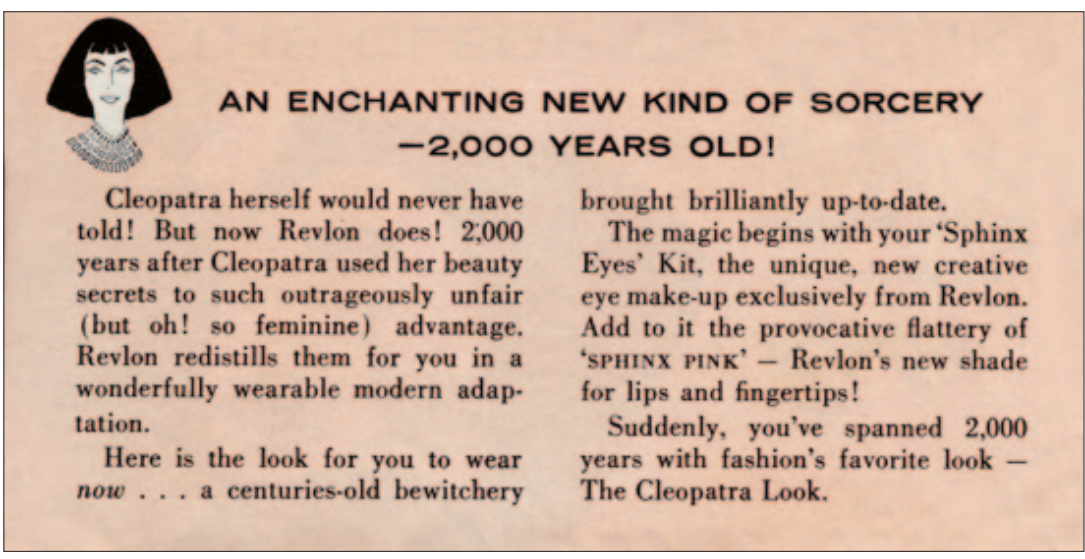

Abb. 3.41 - Gebrauchsanleitung des Revlon Sphinx Eye Makeup 


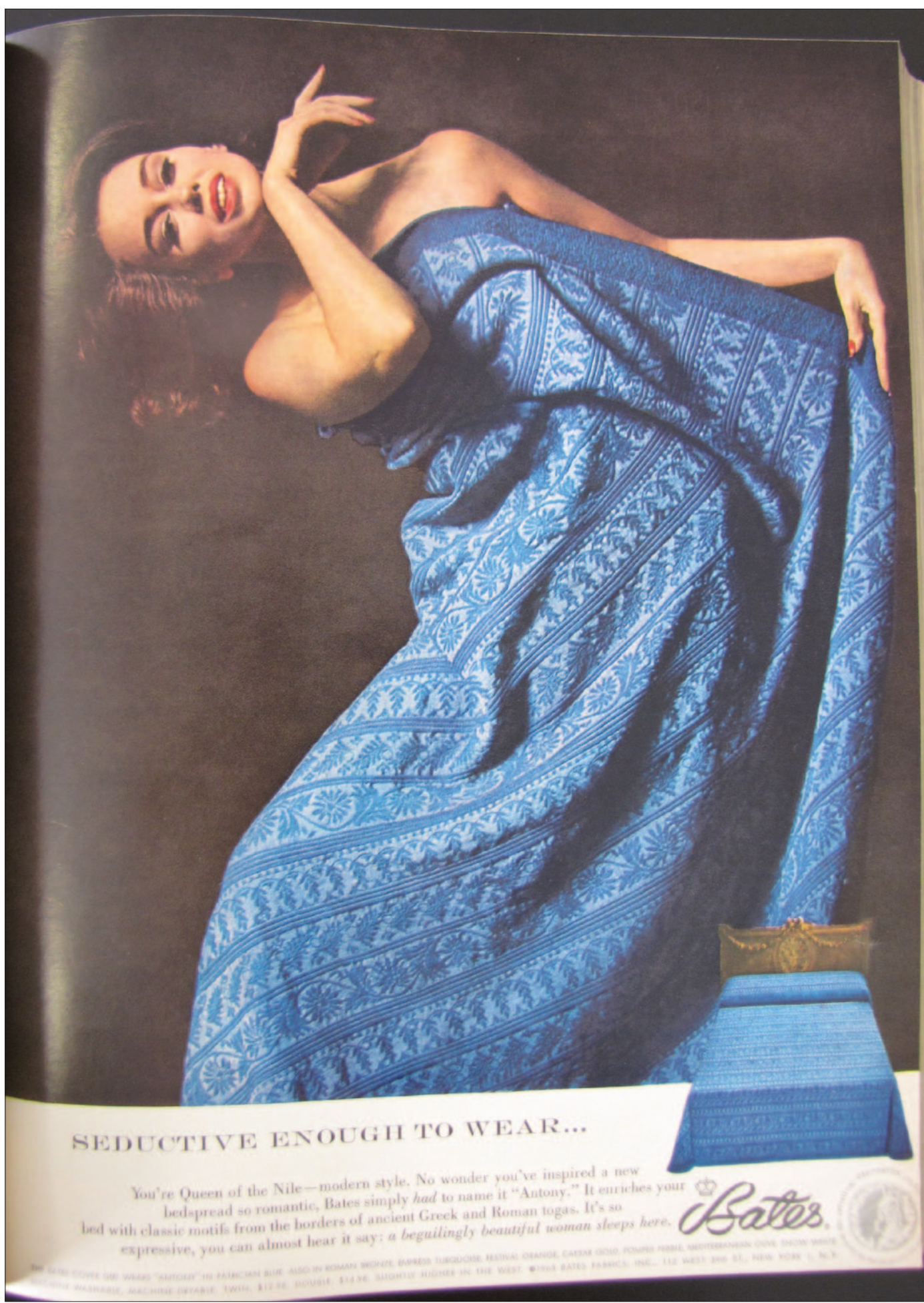

Abb. 3.42 - Anzeige für Bates Fabrics, VOGUE, Vol. 142, Nr. 3 


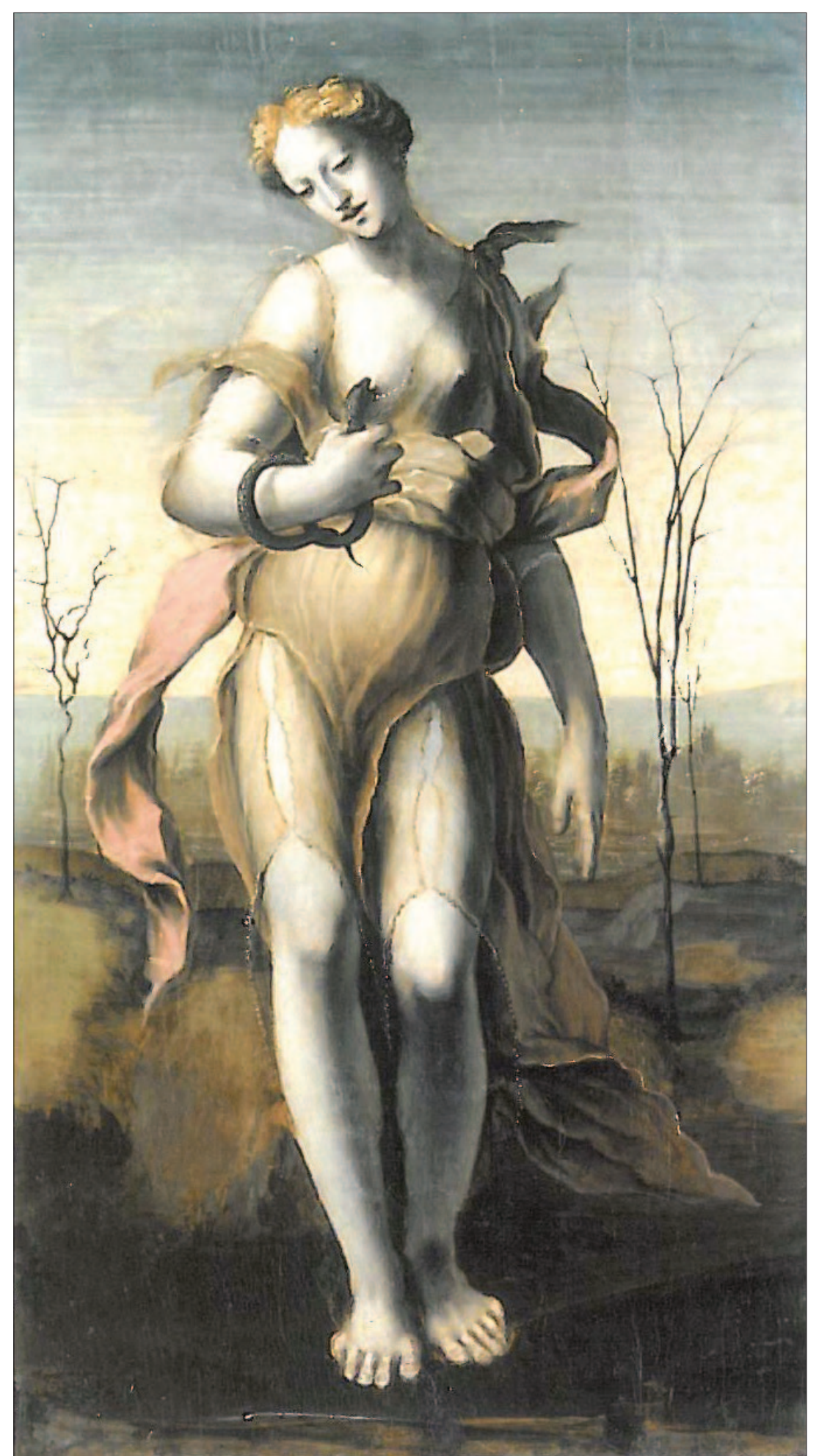

Abb. 3.43 - Domenico Beccafumi: Kleopatra. 1508-1510, Öl auf Holz, 75,5 × 47cm, Musée Bonnat, Bayonne 


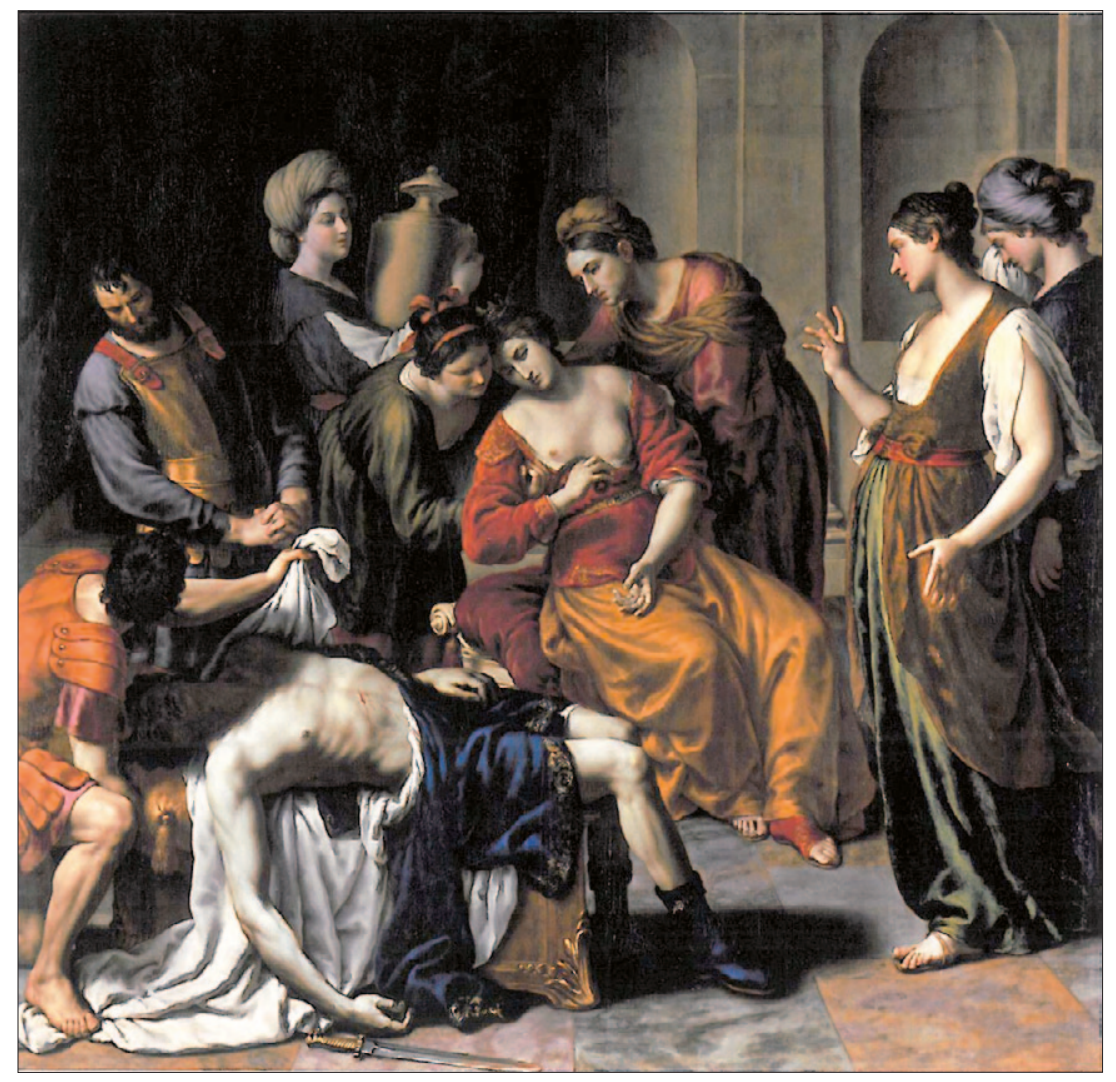

Abb. 3.44 - Alessandro TuRchi: Der Tod der Kleopatra. 1640, Öl auf Leinwand, $255 \times 267 \mathrm{~cm}$, Louvre, Paris 


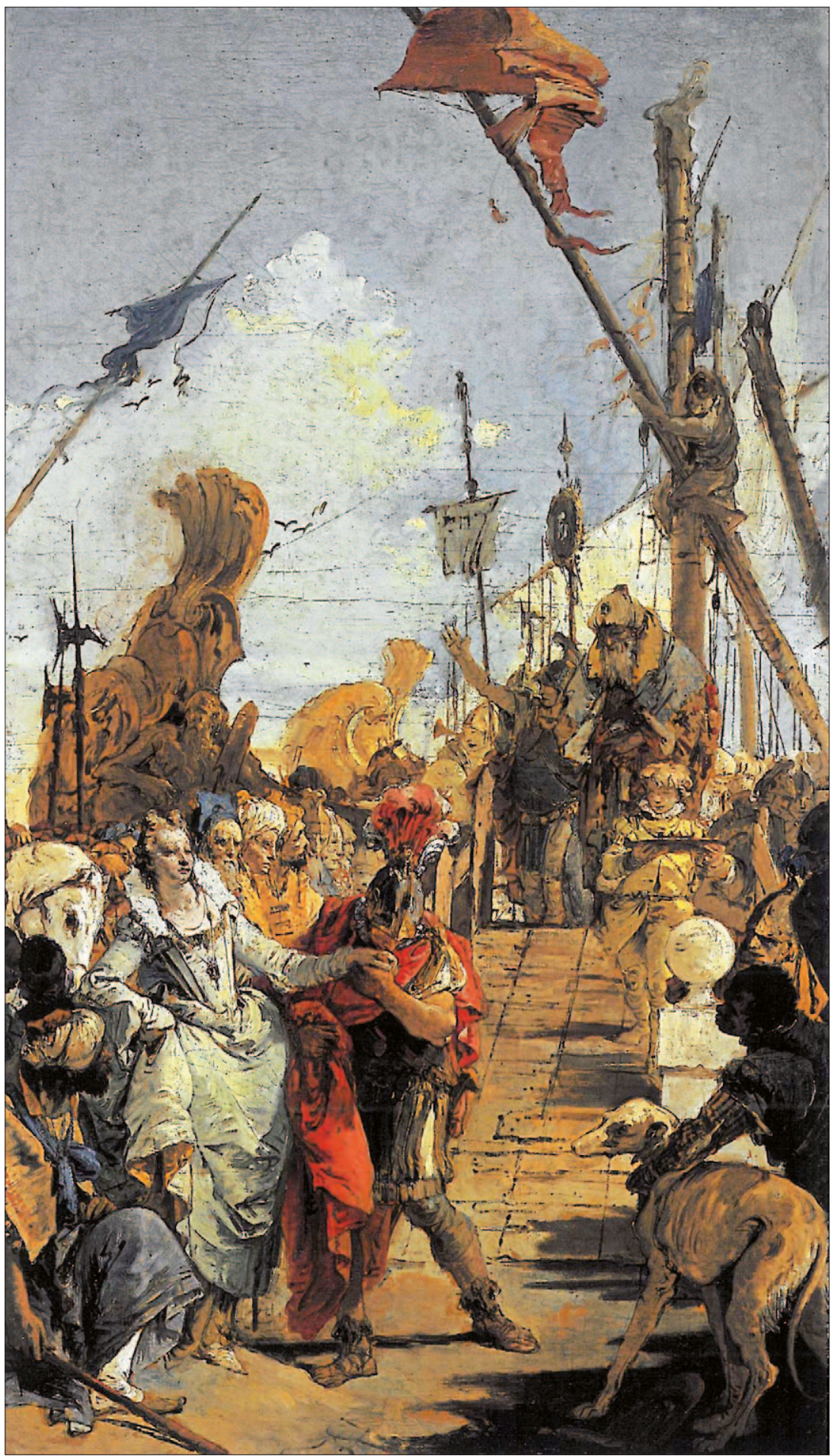

Abb. 3.45 - Giambattista Tiepolo: Das Treffen von Antonius und Kleopatra. ca. 1746, Öl auf Leinwand, $66 \times 38 \mathrm{~cm}$, National Gallery of Scotland, Edinburgh 


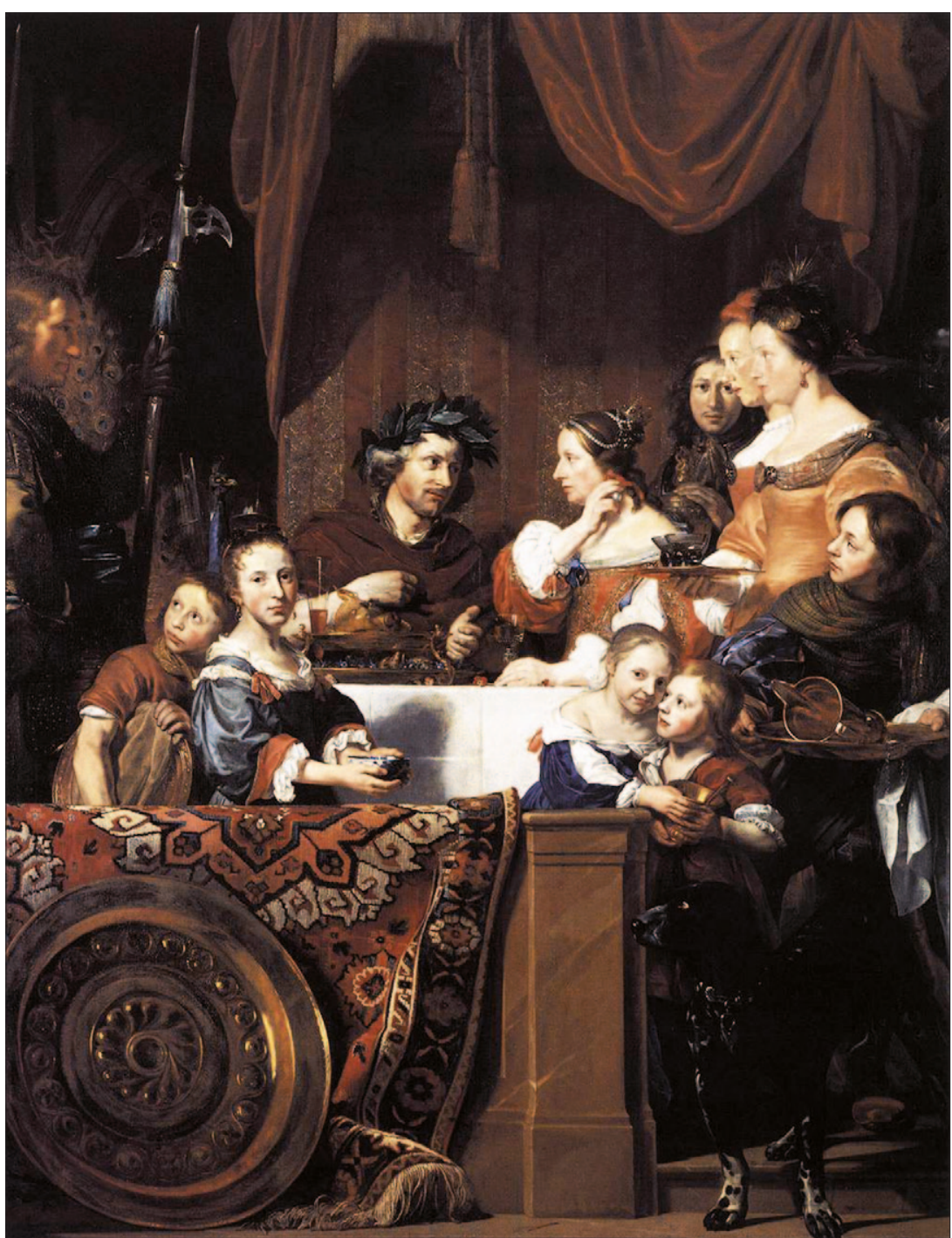

Abb. 3.46 - JAN DE BRAY: Das Bankett von Antonius und Kleopatra. 1669, Öl auf Leinwand, 248,9 × 190,5 cm, The Currier Gallery of Art, Manchester, New Hampshire 


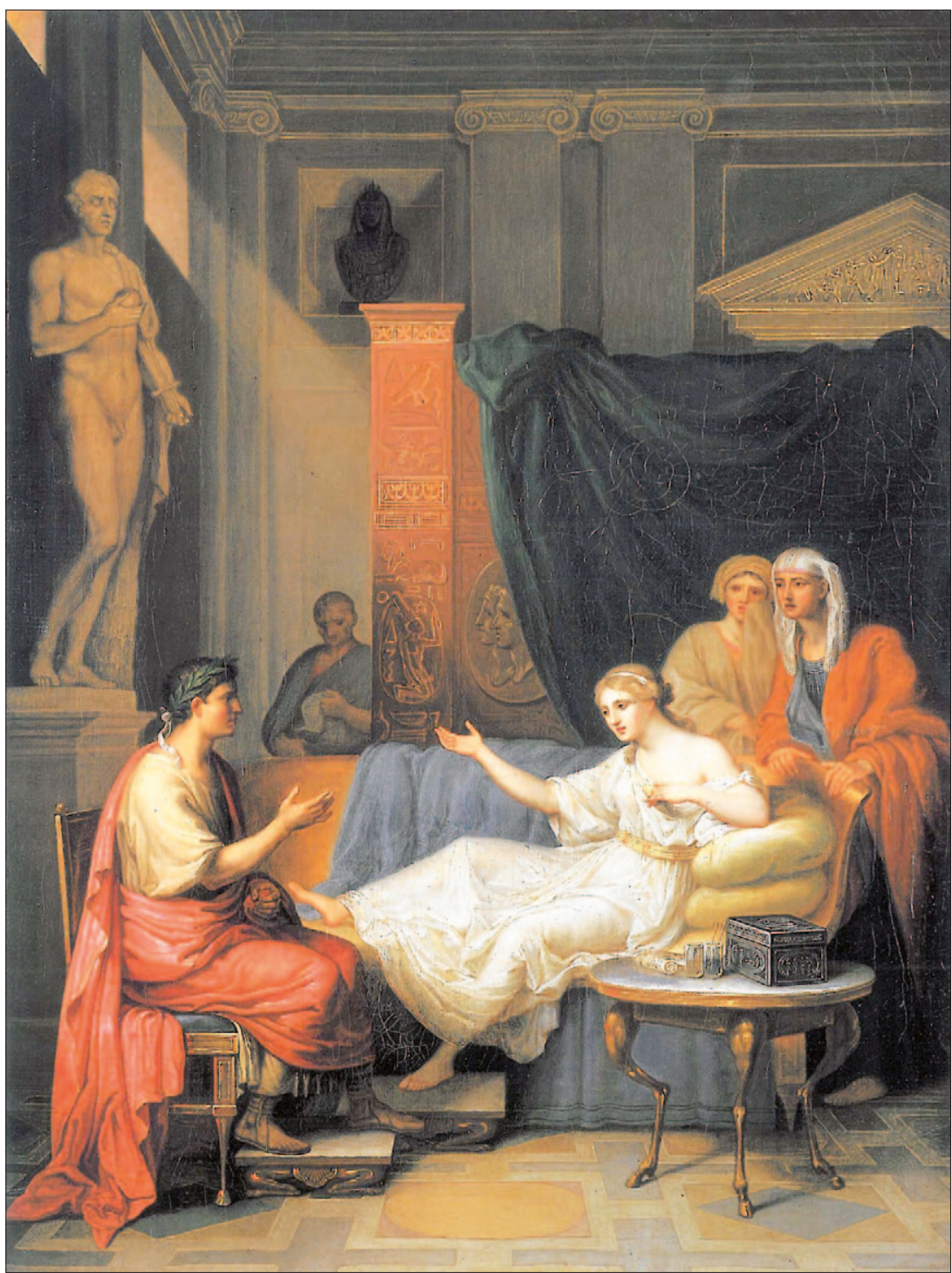

Abb. 3.47 - Anton Raphael Mengs: Augustus und Kleopatra. 176o, Öl auf Leinwand, $306 \times 212 \mathrm{~cm}$, Stourhead House, Wiltshire

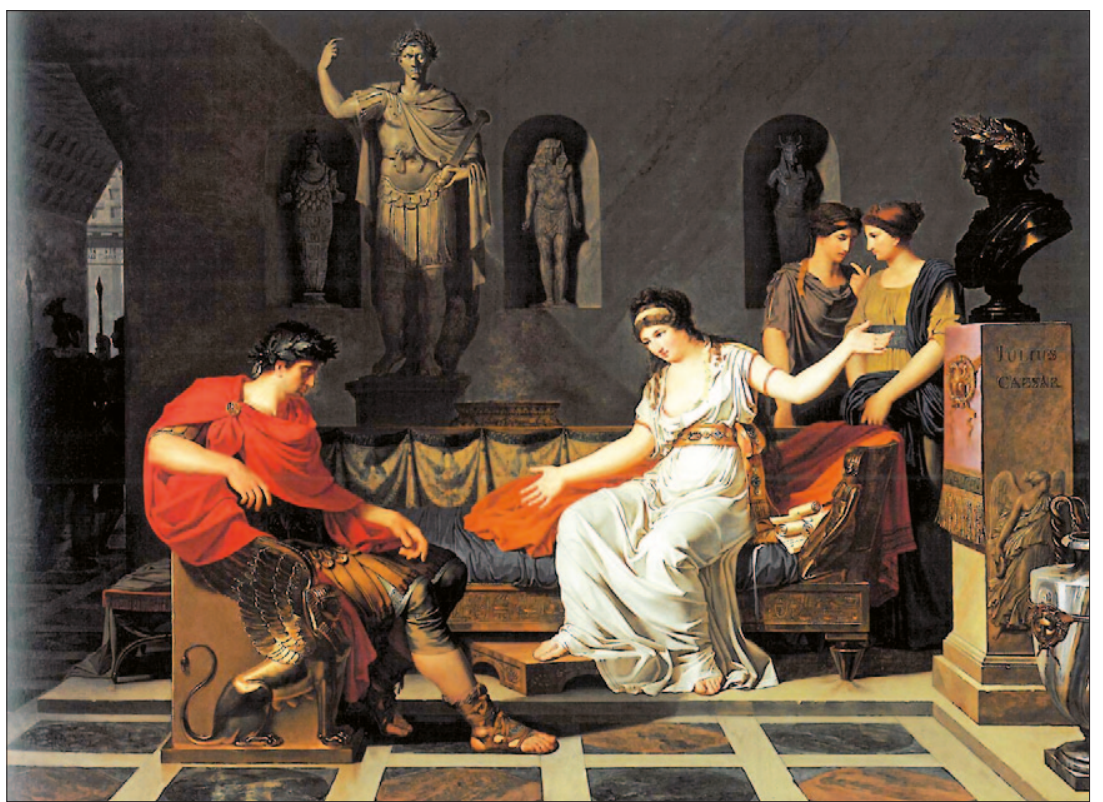

Abb. 3.48 - Louis GAuffier: L'Entrevue d'Auguste et de Cléopâtre après la Bataille d'Actium. 1788, Öl auf Leinwand, 83,8 × 112,5 cm, National Gallery of Scotland, Edinburgh 


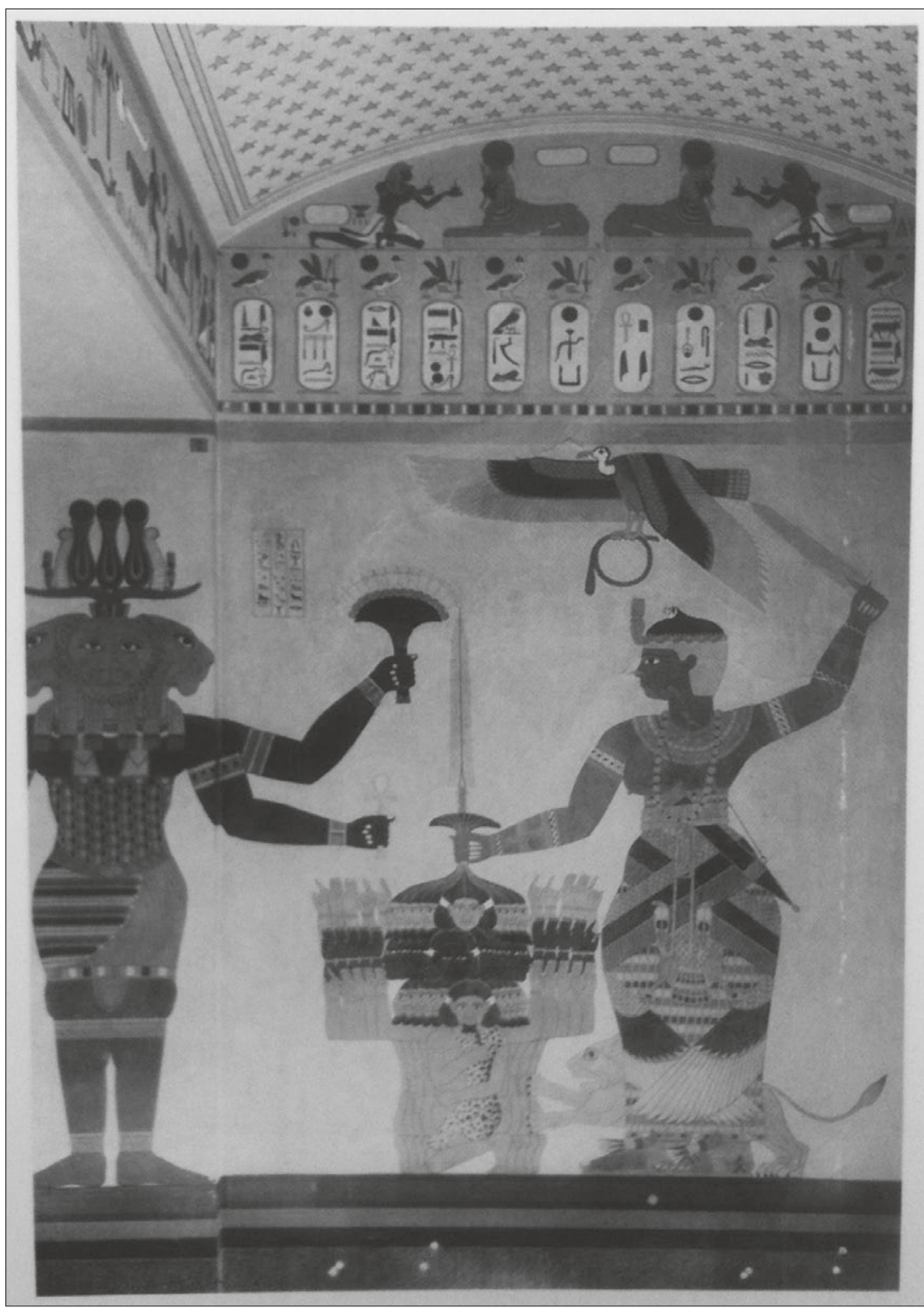

Abb. 3.49 - Hist. Foto der Wandbemalung im Neuen Museum Berlin. Messling, 1997, Die ägyptische Abteilung im Neuen Museum zu Berlin, Abb. 9, S. 83 


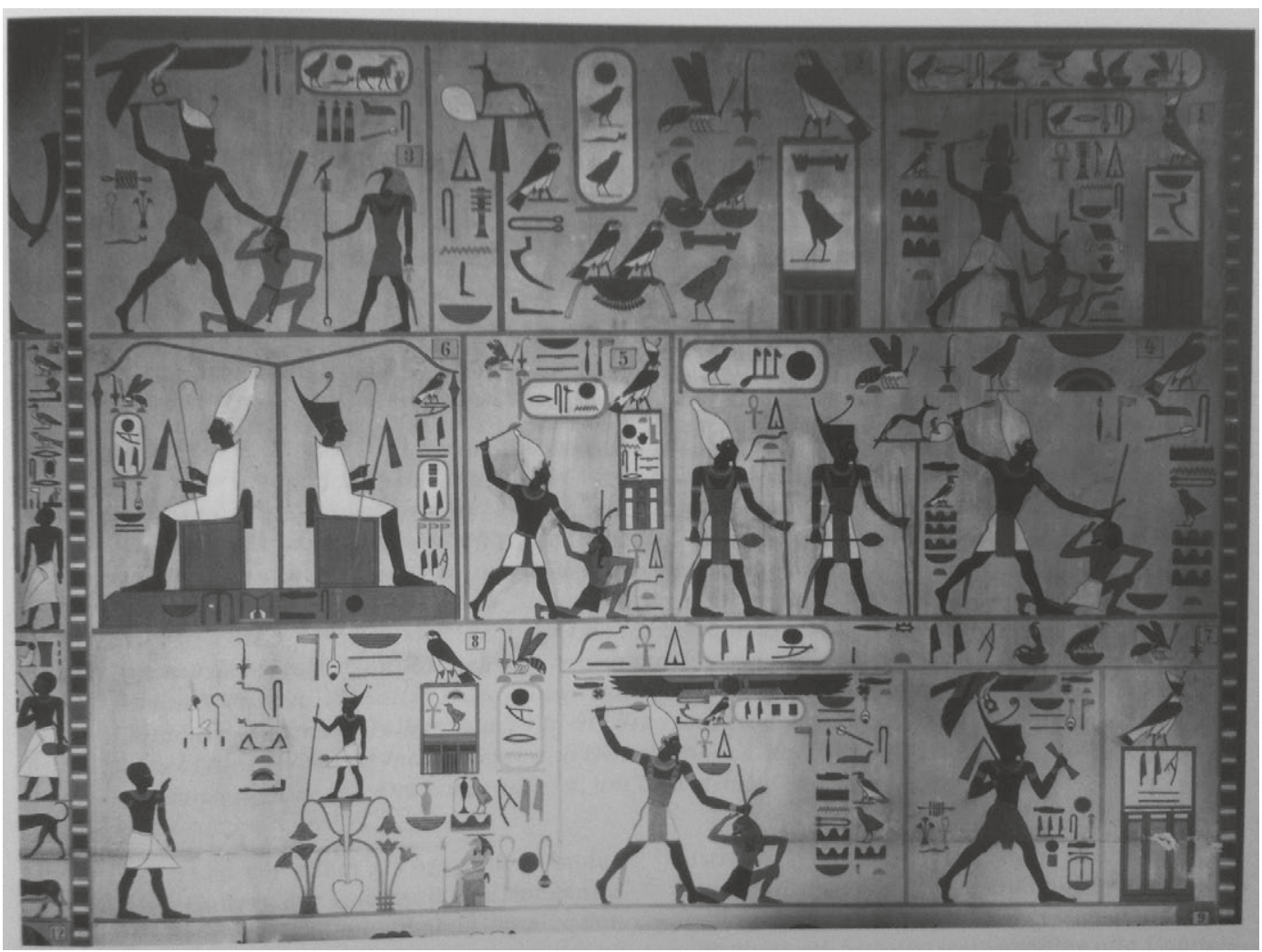

Abb. 3.50 - Hist. Foto der Wandbemalung im Neuen Museum mit Bezifferung neben den Kartuschen. Messling, 1997, Die ägyptische Abteilung im Neuen Museum zu Berlin, Abb. 17, S. 97

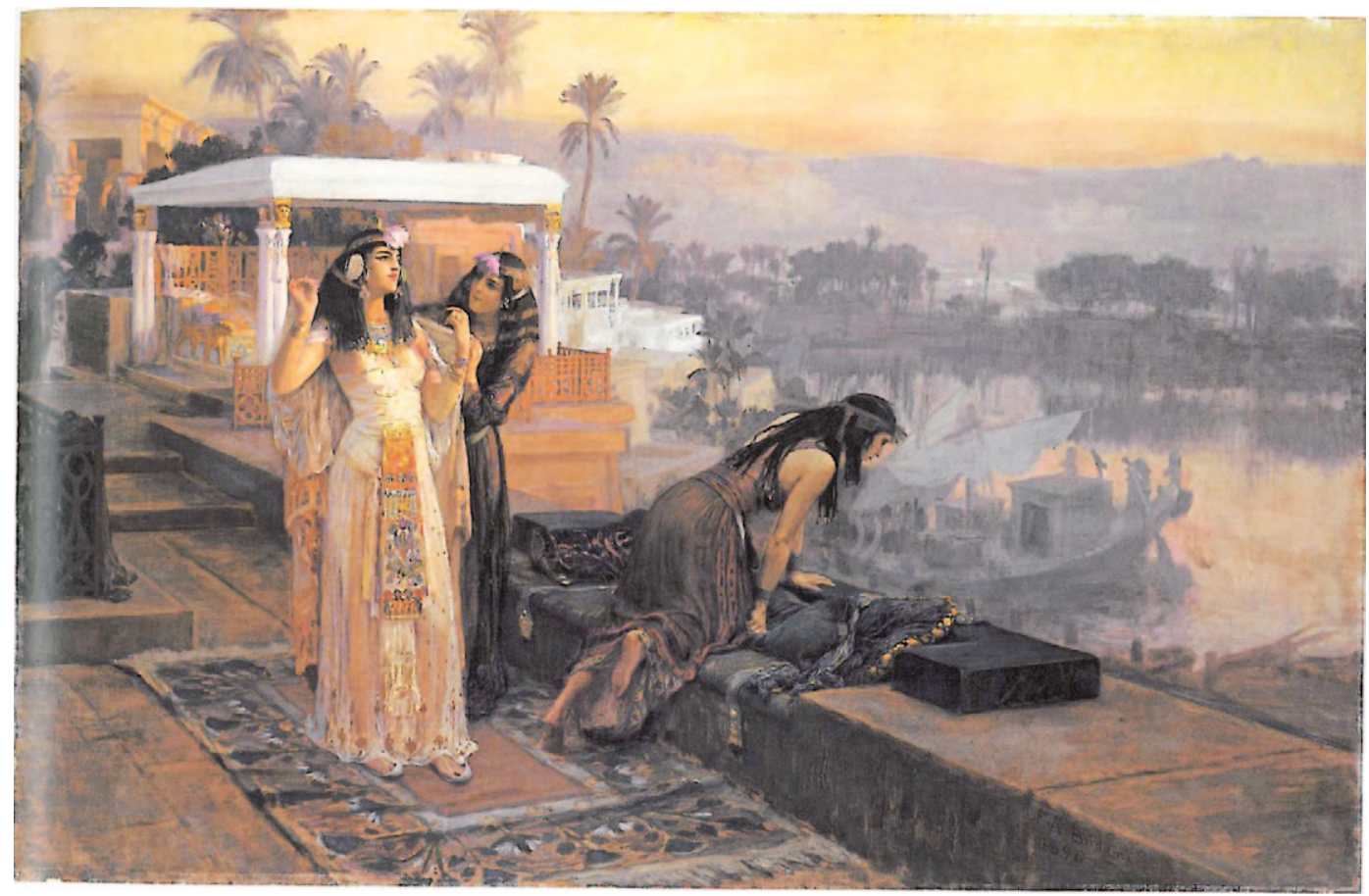

Abb. 3.51 - FREDERICK ARTHUR BRIDgman: Kleopatra auf den Terrassen von Philae. 1896, Öl auf Leinwand, 75,9 × 117,2 cm, Dahesh Museum of Art, New York 




Abb. 3.52 - Georges Antoine Rochegrosse: Altes Ägypten oder Kleopatra und ihr Gefolge. ca. 189o, Öl auf Leinwand, $65 \times 82 \mathrm{~cm}$, Privatsammlung

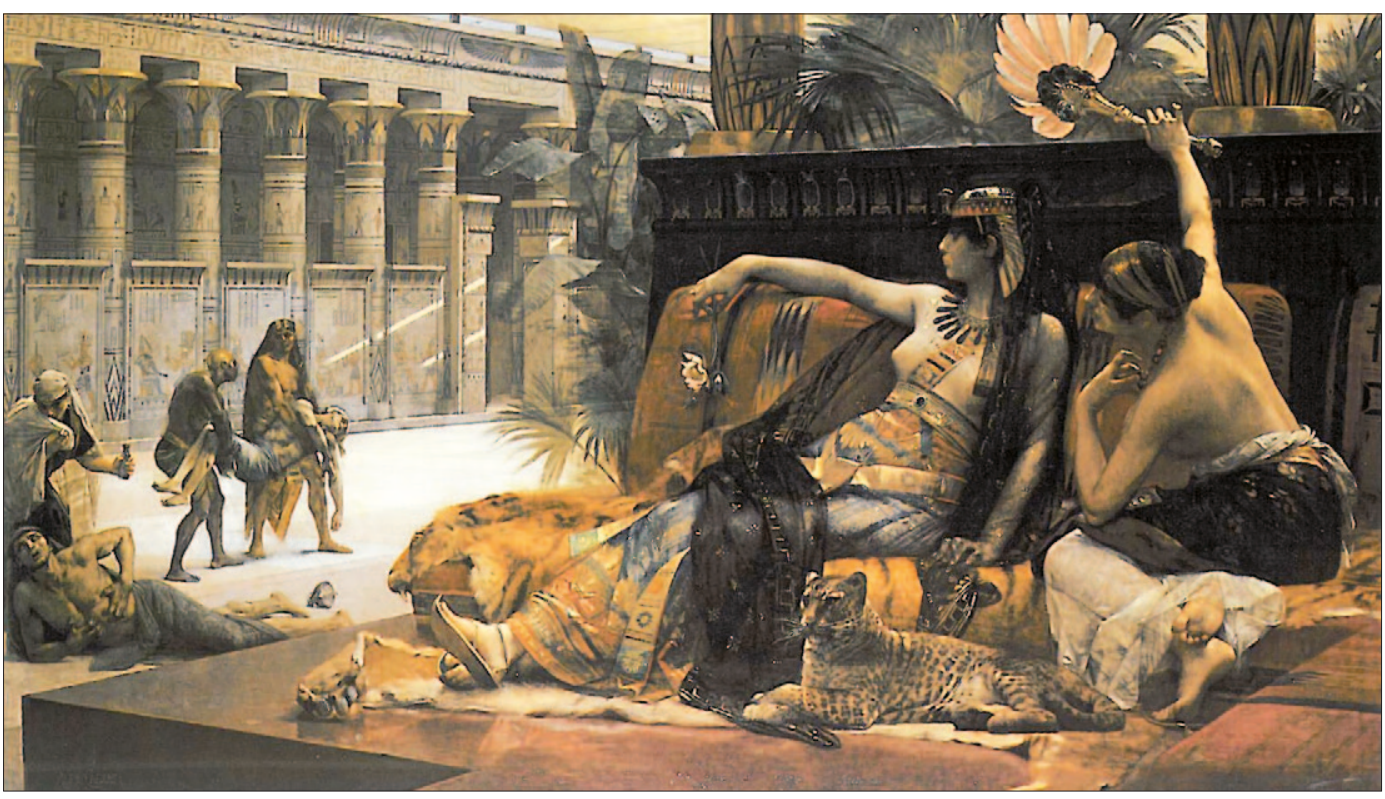

Abb. 3.53 - AleXANDRe CABANEL: Kleopatra erprobt die Wirkung des Giftes an zum Tode Verurteilten. 1887, Öl auf Leinwand, $165 \times 290 \mathrm{~cm}$, Koninklijk Museum voor schone Kunsten, Anvers 


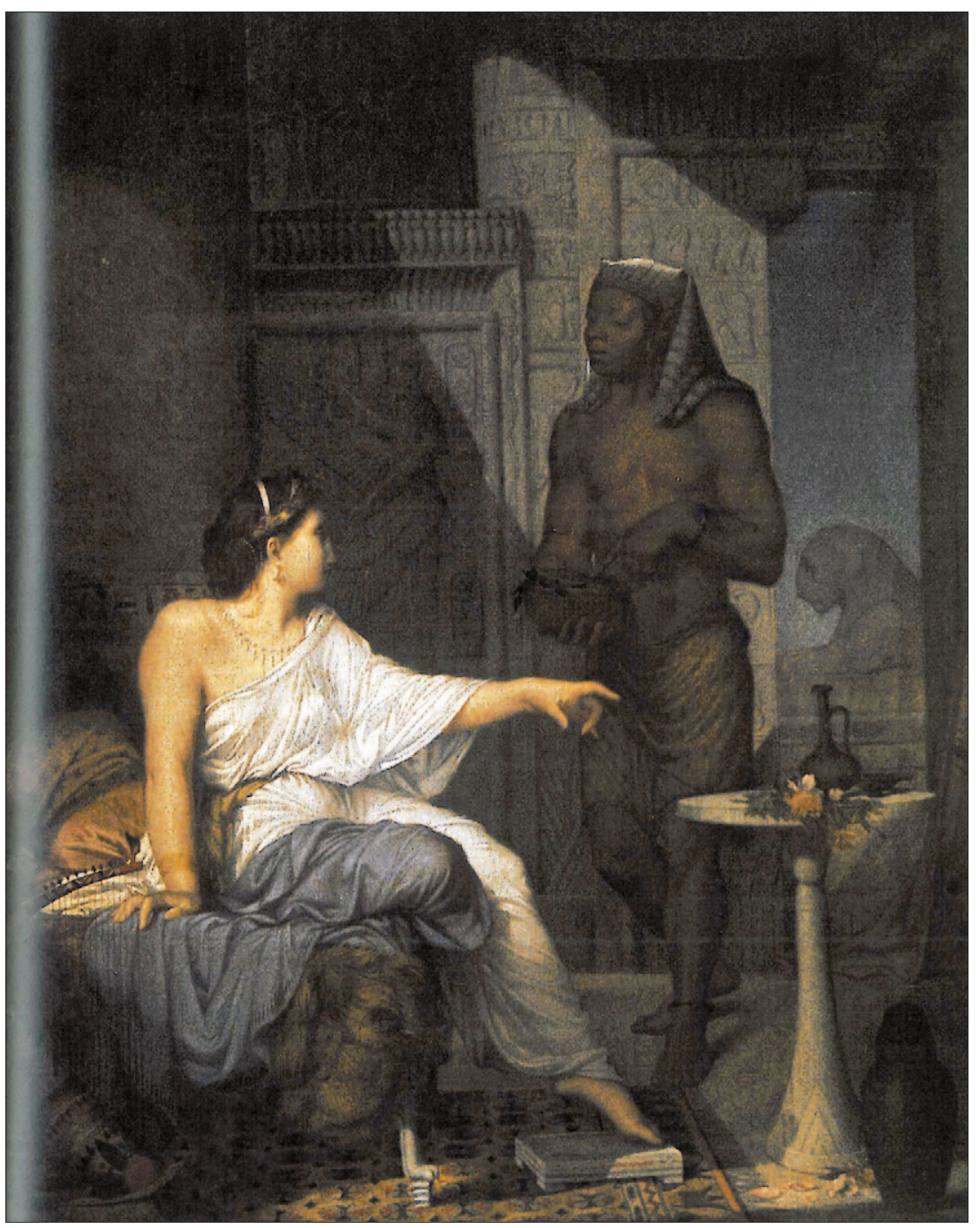

Abb. 3.54 - Henri Blaise François DeJUssieu: Kleopatra und der Sklave. 1863, Öl auf Leinwand, 82 × 66 cm, Musée Denon, Chalon-sûr-Saône

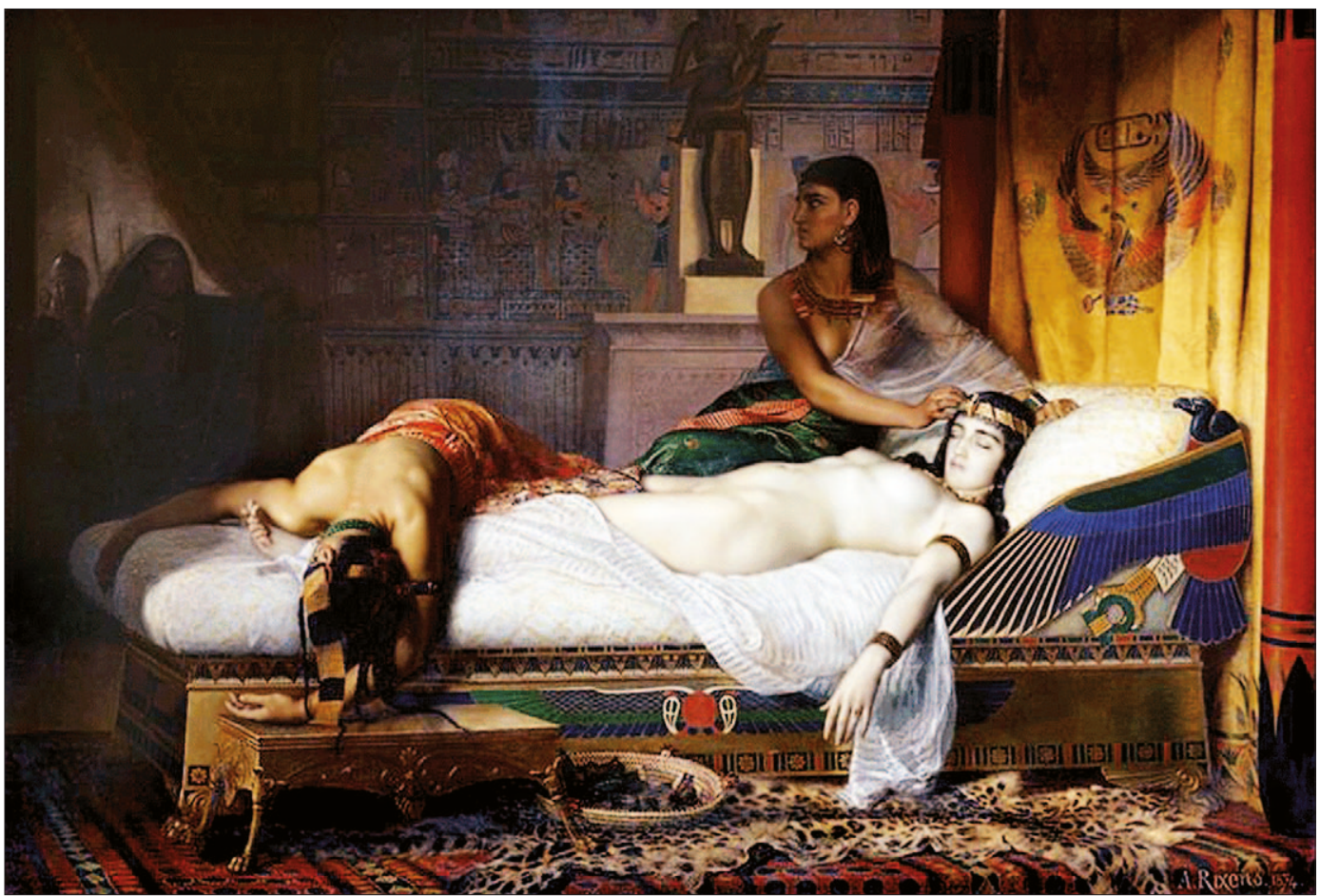

Abb. 3.55 - JeAn AndRÉ Rixens: Der Tod der Kleopatra. 1874, Öl auf Leinwand, 200 × $290 \mathrm{~cm}$, Musée des Augustins, Toulouse 


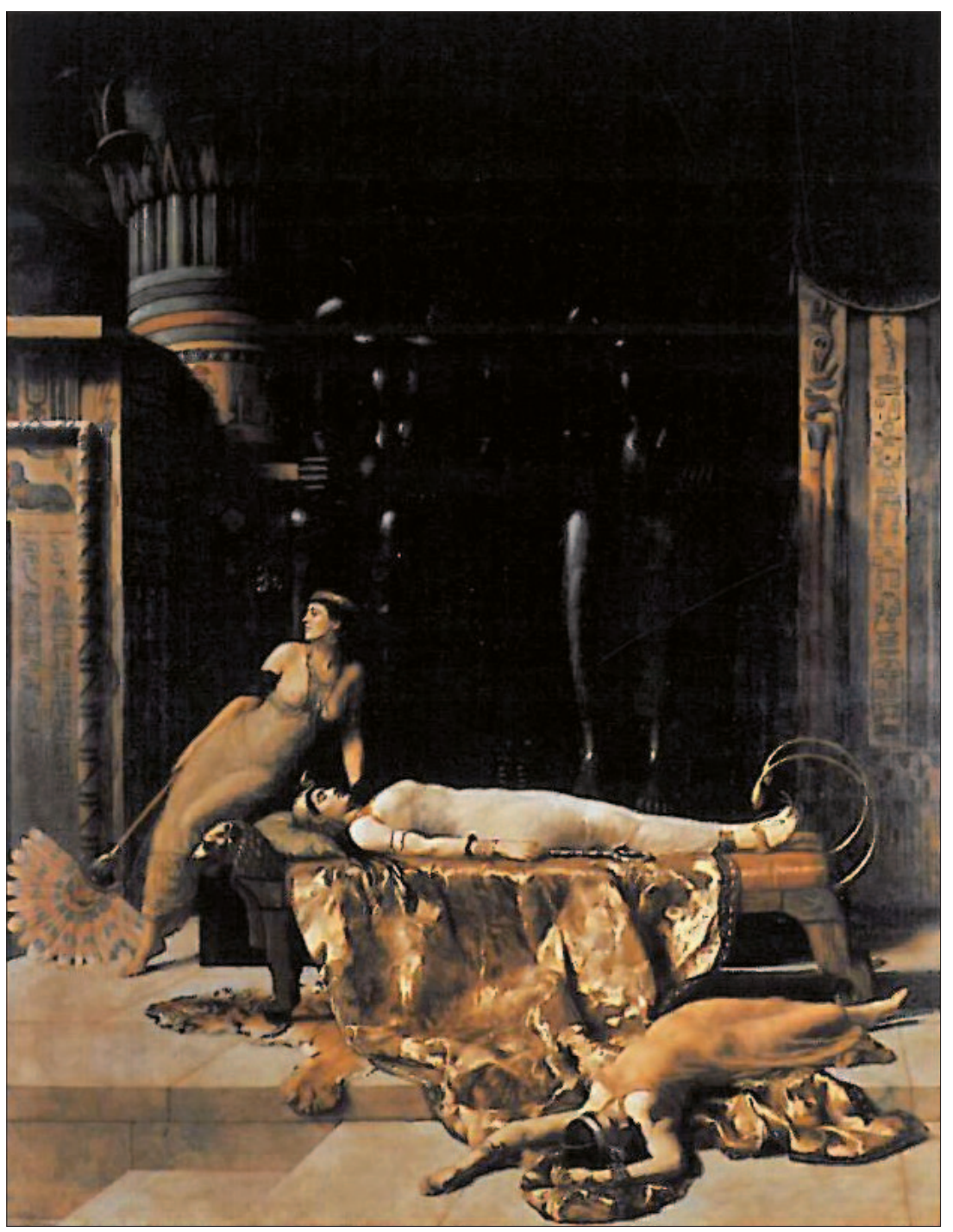

Abb. 3.56 - John ColLIeR: Der Tod der Kleopatra. 189o, Öl auf Leinwand, $395 \times 315 \mathrm{~cm}$, Gallery Oldham, Oldham 


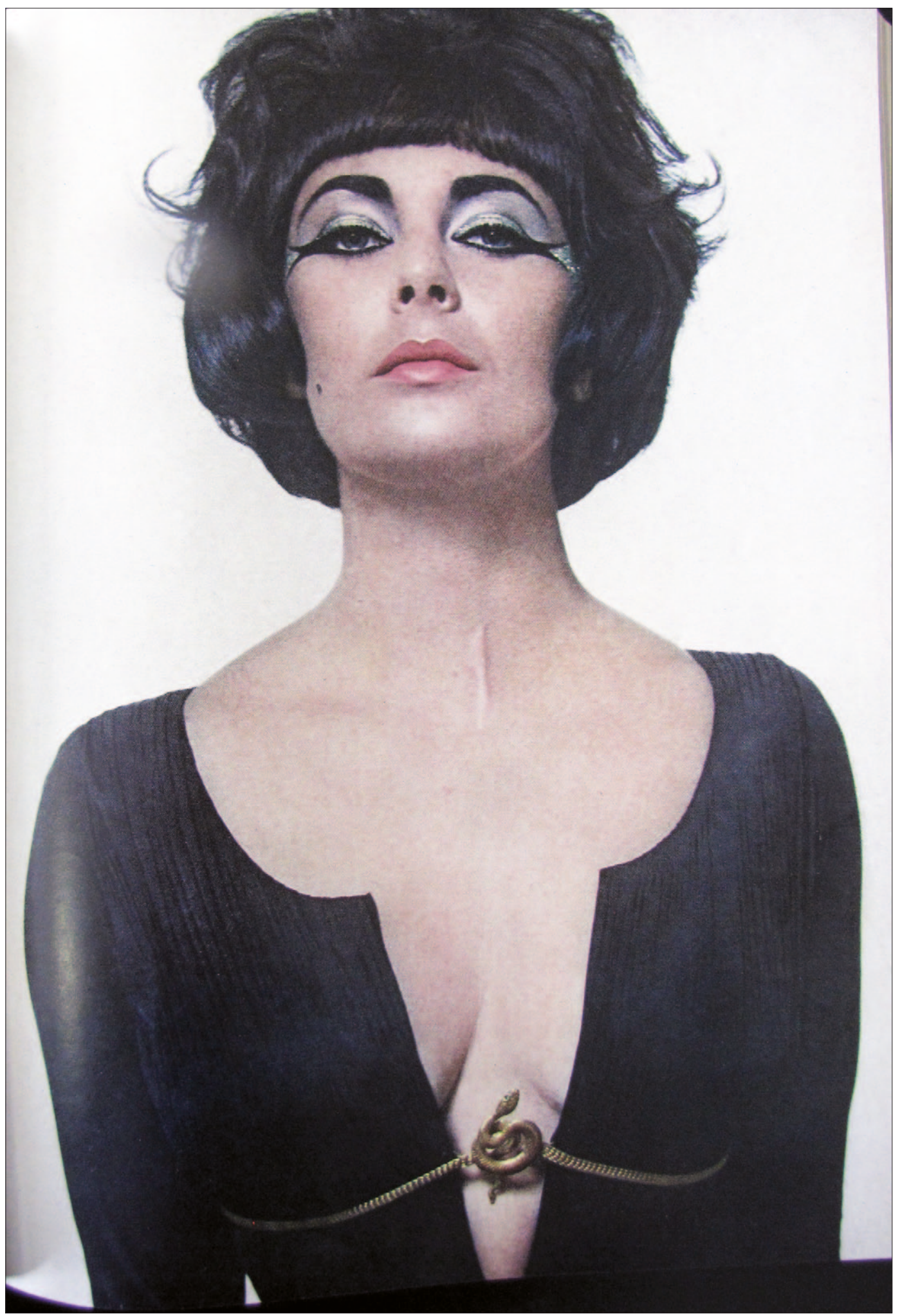

Abb. 3.57 - Elizabeth Taylor als Cleopatra in VOGUE, Vol. 139, Nr. 2, S. 71 


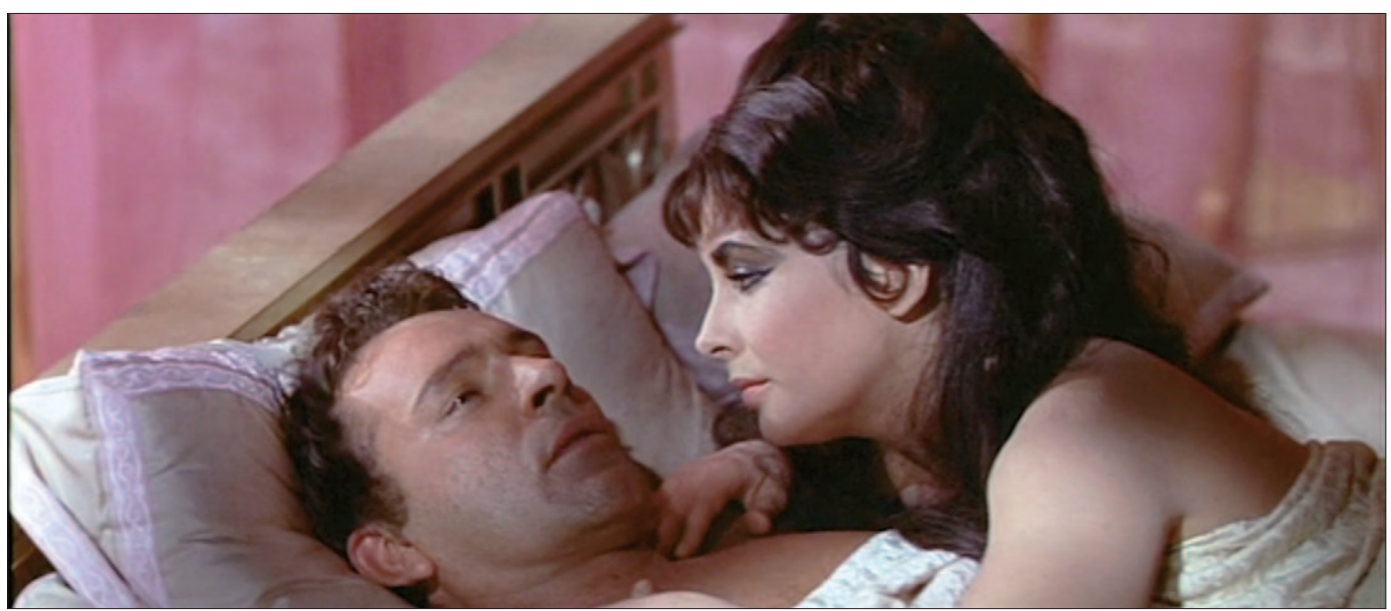

Abb. 3.58 - Cleopatras snatürlicher Look`, CLEOPATRA 
Abbildungen Kapitel 4

Die Nummerierung der Sequenzen der Abbildungen aus CARAVAggio folgt derjenigen in Derek Jarman, 1986, Derek Jarman's Caravaggio. London.

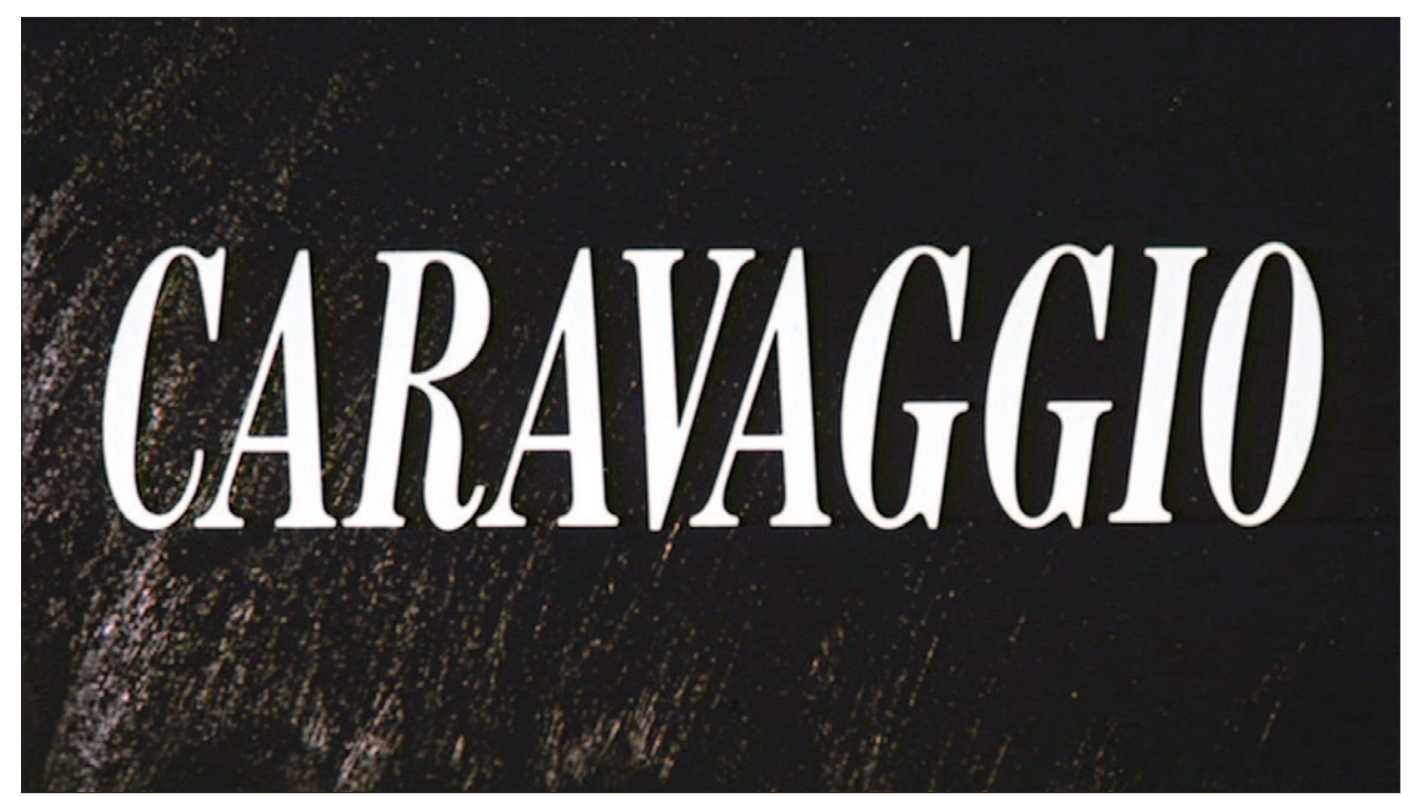

Abb. 4.1 - Einst. aus der Titelsequenz von CARAVAgGIO

\section{SEAN BEAN} GARRY COOPER DEXTER FLATCHER SPENCDR LEIGH TILDA SWINTON

Abb. 4.1 - Einst. aus der Titelsequenz von CARAVAGgiO 


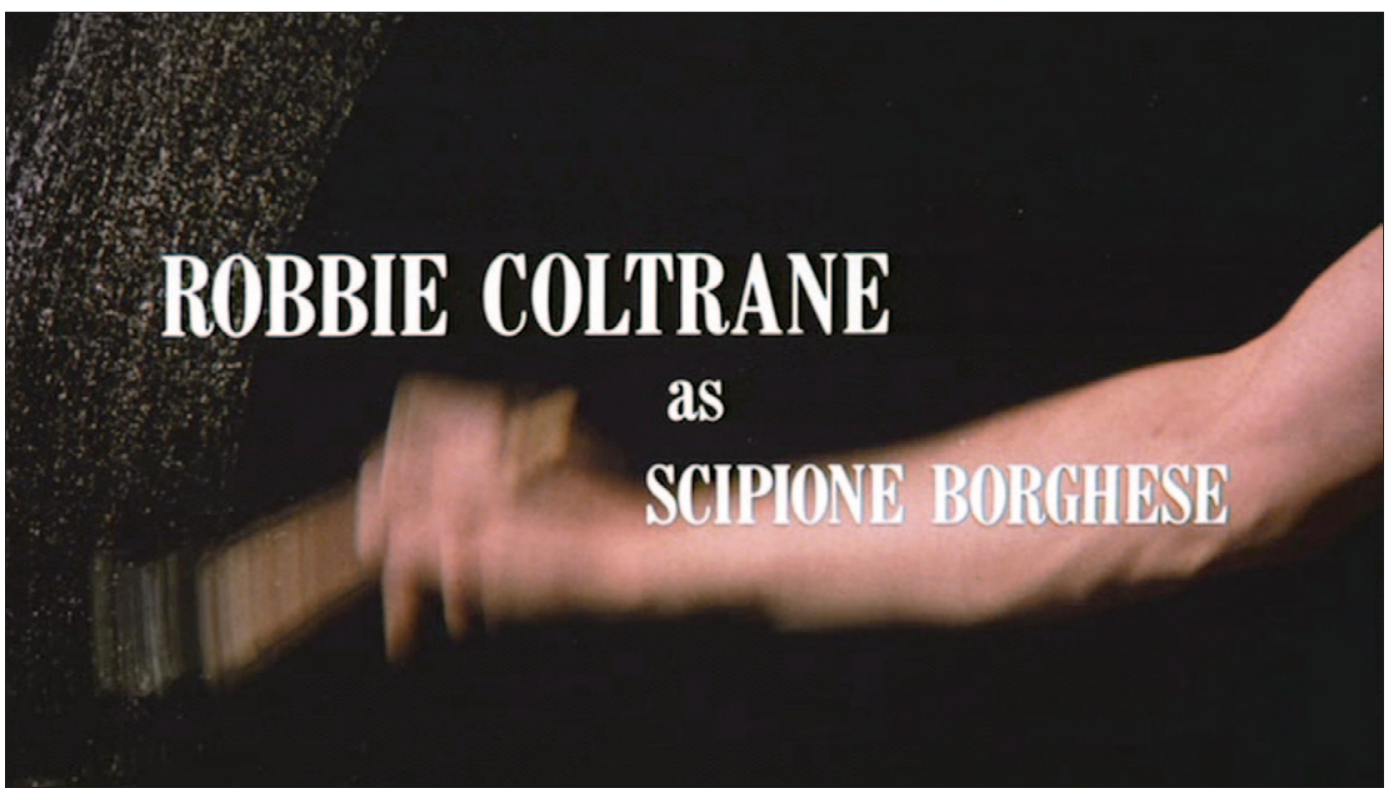

Abb. 4.3 - Einst. aus der Titelsequenz von CARAVAggio

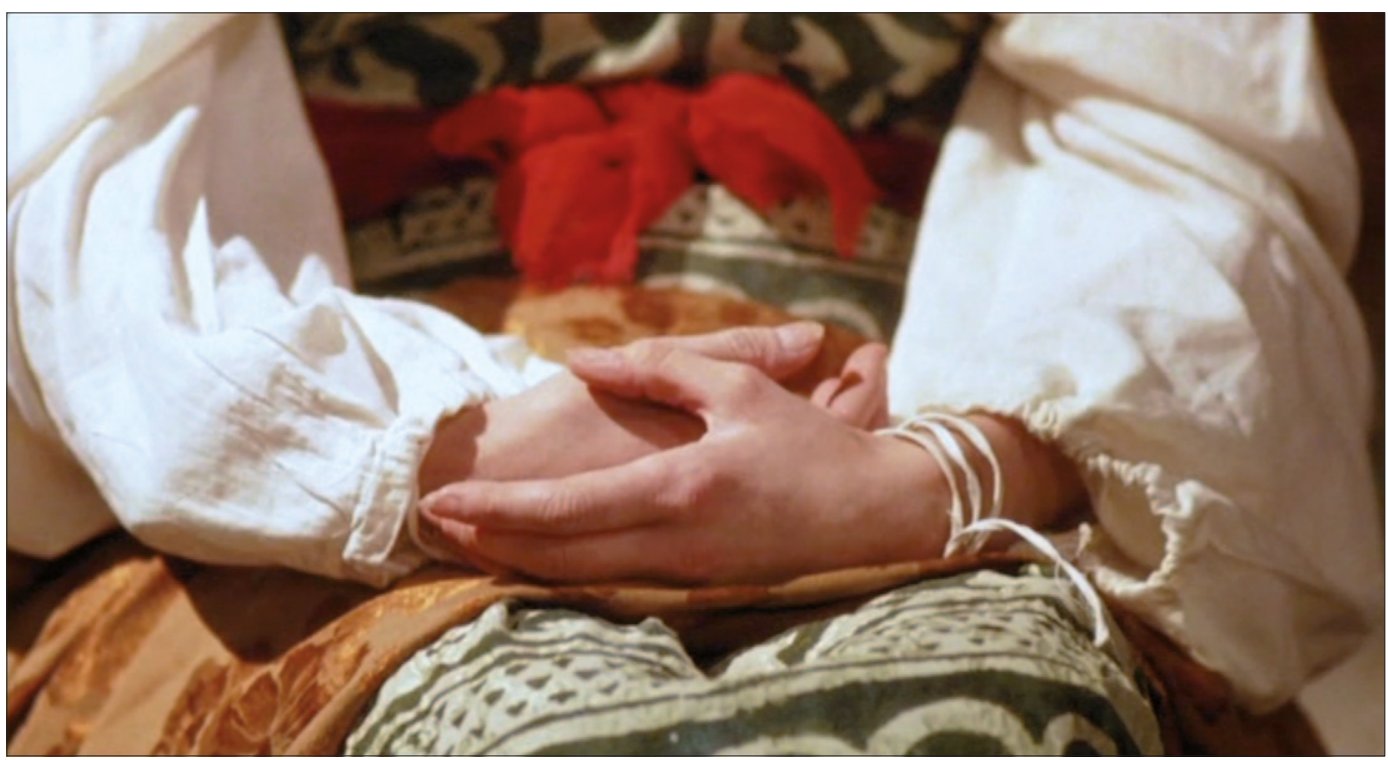

Abb. 4.4 - Einst. 1 nach Sequenzprotokoll 4, CARAVAGgIO

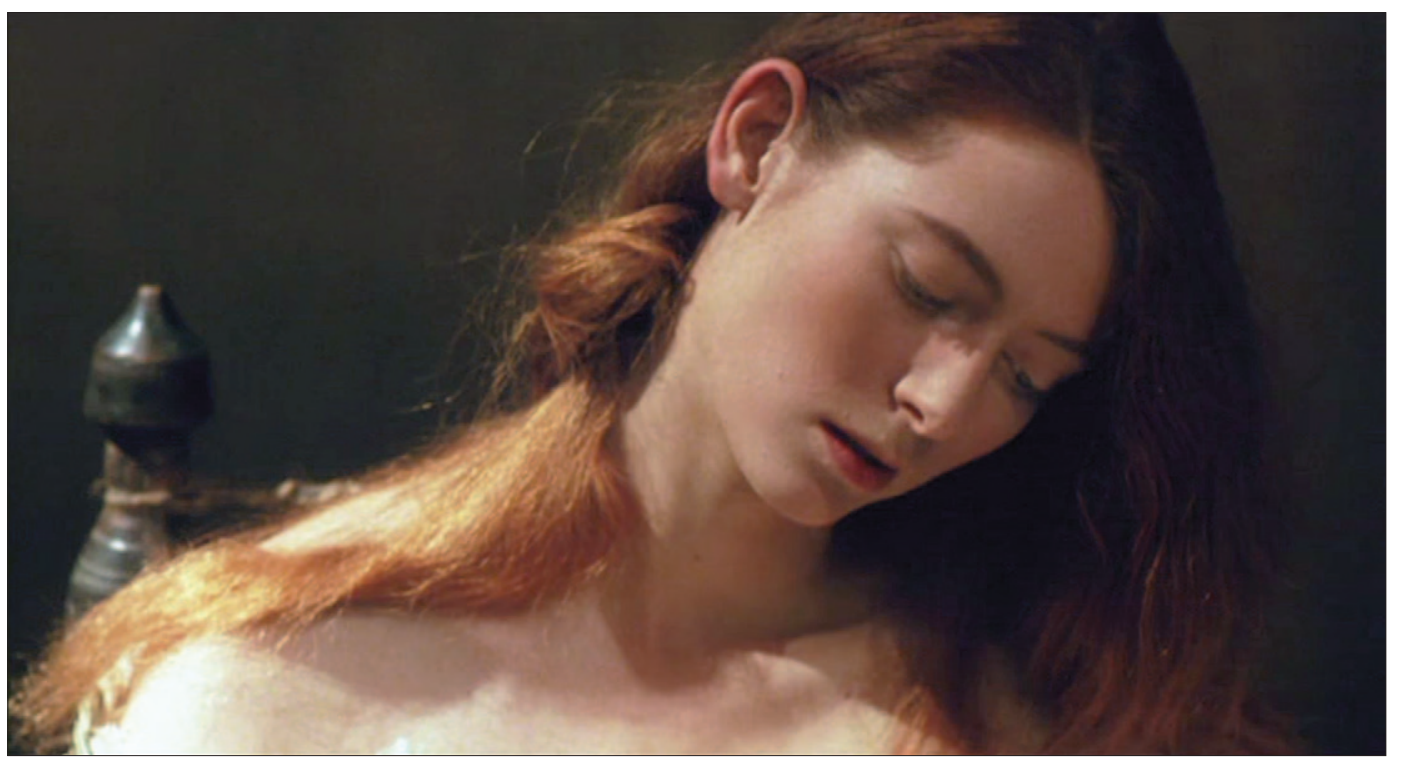

Abb. 4.5 - Einst. 2 nach SP 4, CARAVAgGIO 


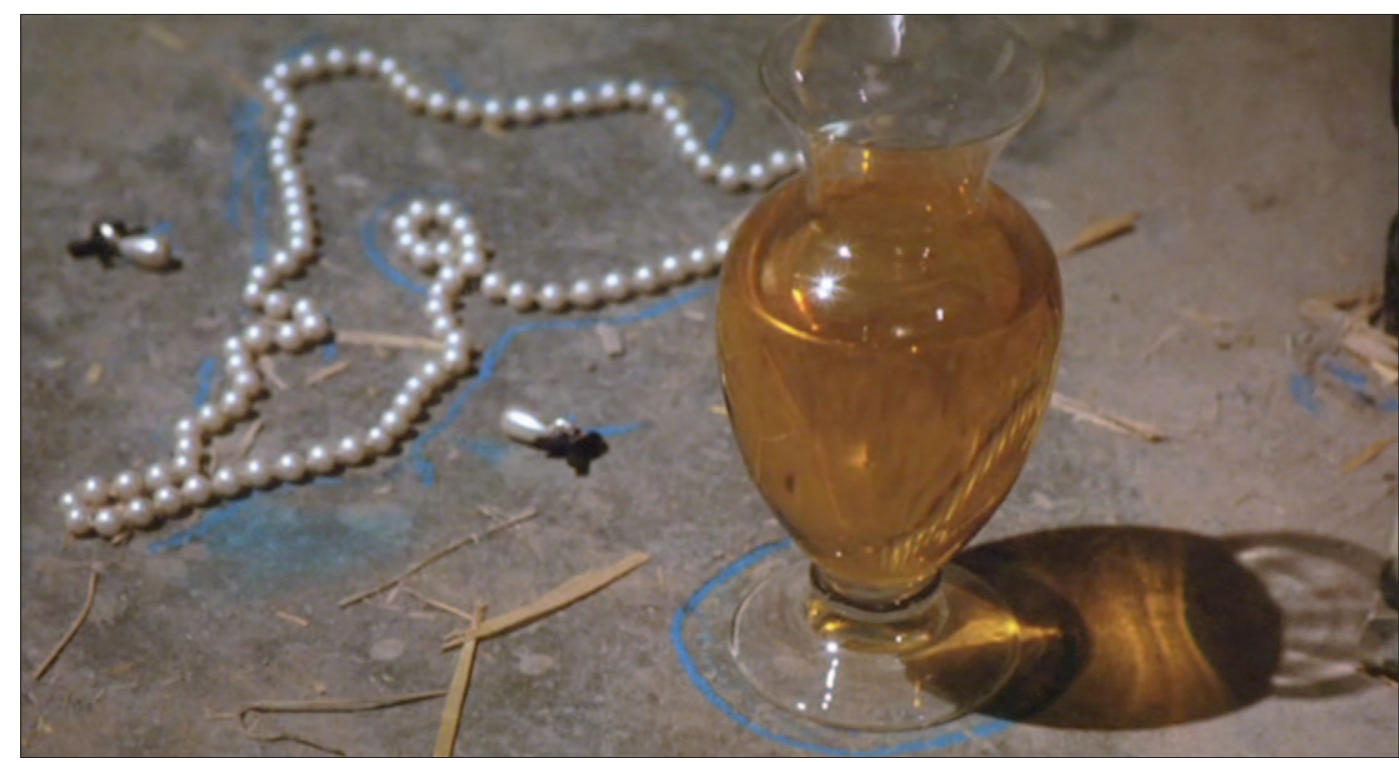

Abb. 4.6 - Einst. 3 nach SP 4, CARAVAgGiO

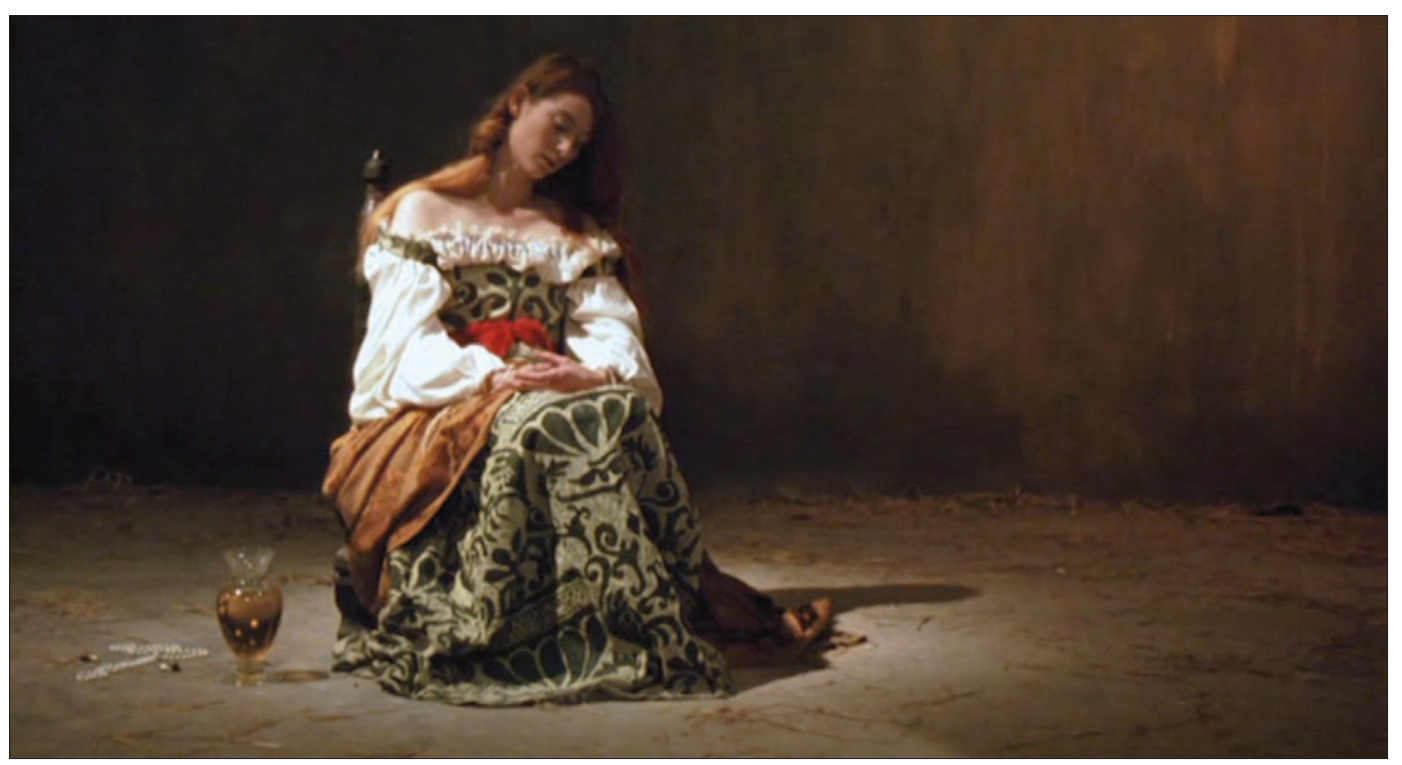

Abb. 4.7 - Einst. 4 nach SP 4, CARAVAGgIO 


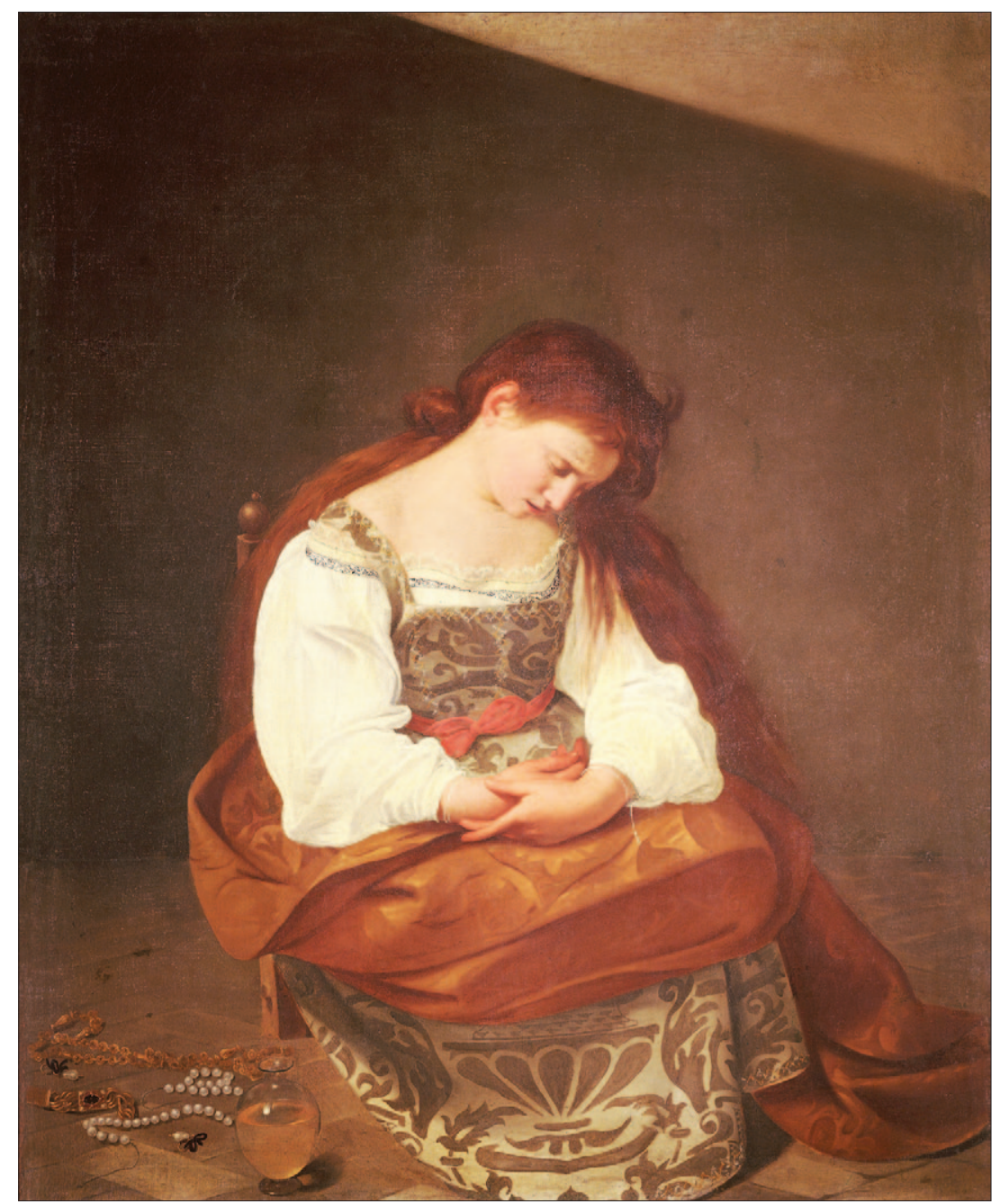

Abb. 4.8 - Caravaggio: Reuige Magdalena. 1594, 122,5 cm × 98,5 cm, Galleria Doria Pamphilj, Rom.

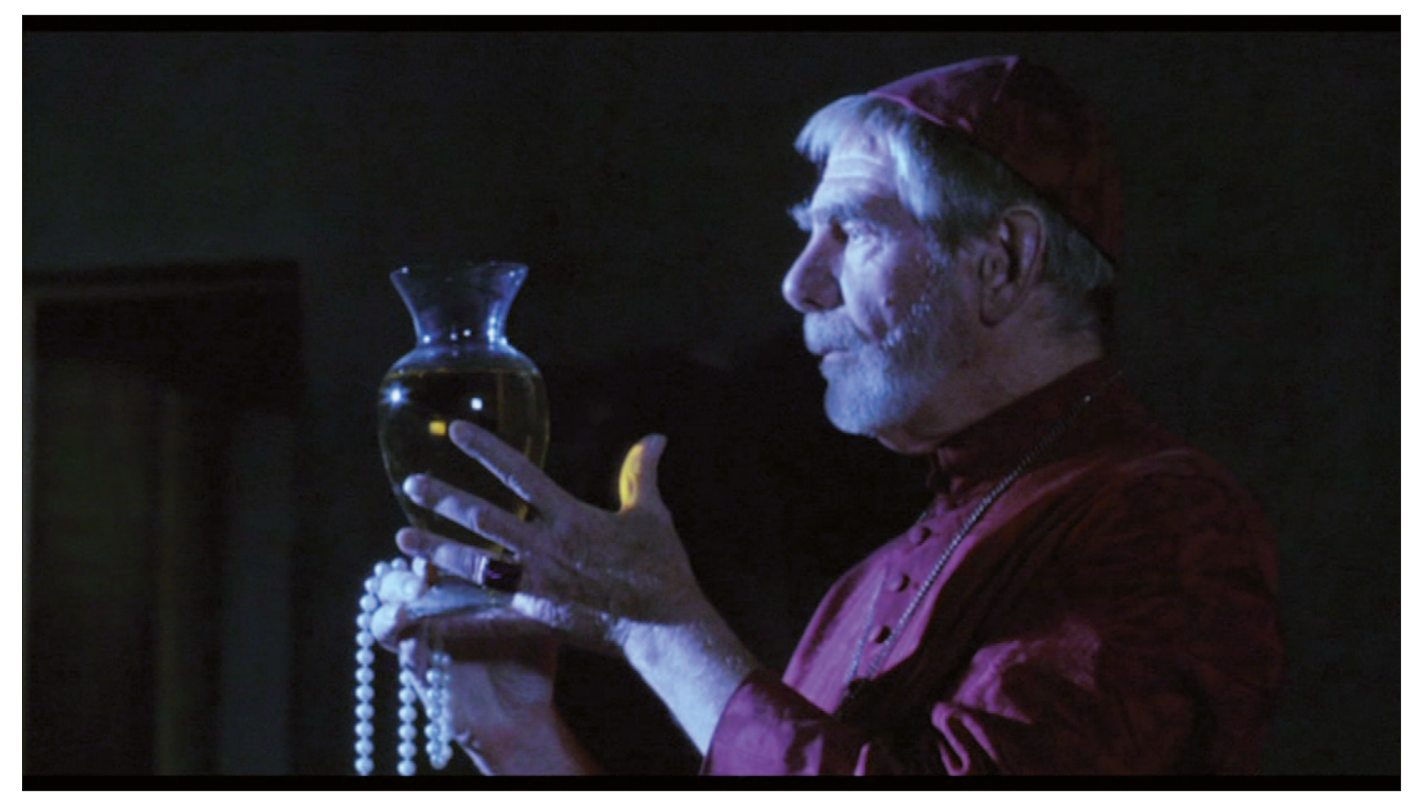

Abb. 4.9 - Einst. 30 nach SP 4, CARAVAGgio 


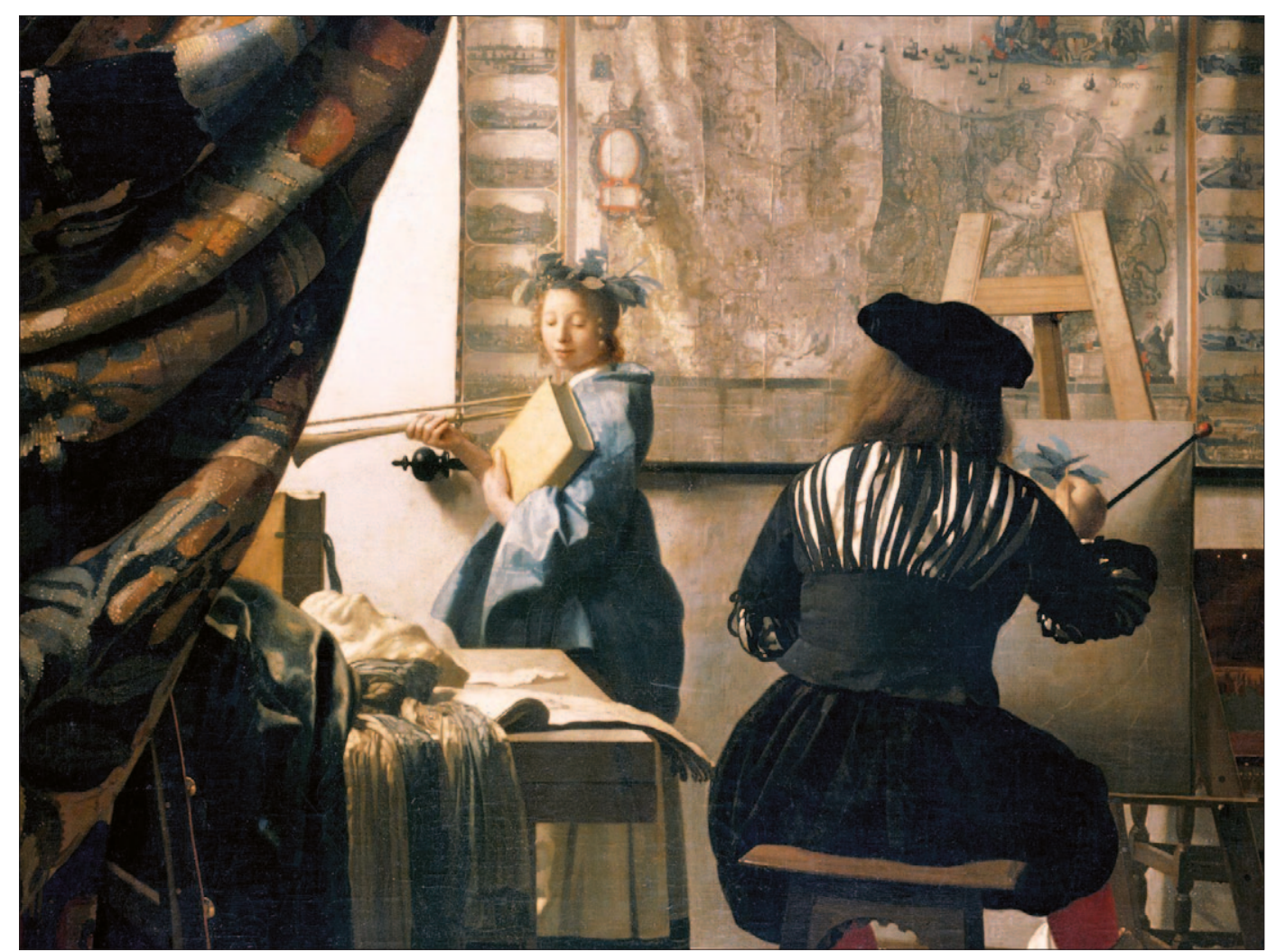

Abb. 4.10 - Johannes Vermeer: Allegorie der Malerei. ca. 1666-68, Öl auf Leinwand, $120 \mathrm{~cm} \times 100 \mathrm{~cm}$, Kunsthistorisches Museum, Wien.

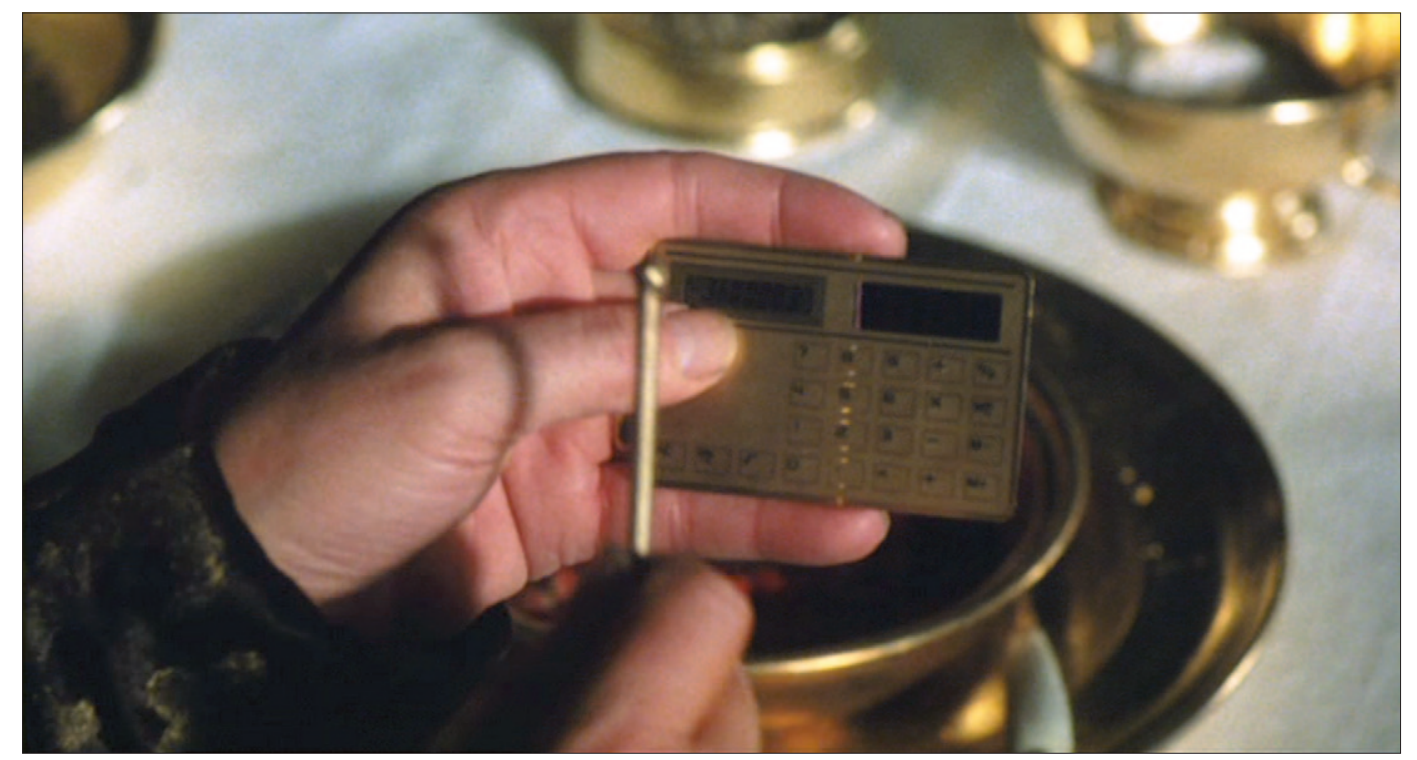

Abb. 4.11 - Vincenzo Giustiniani mit einem Taschenrechner. Einst. aus CARAVAGgIO, Sequ. 27 


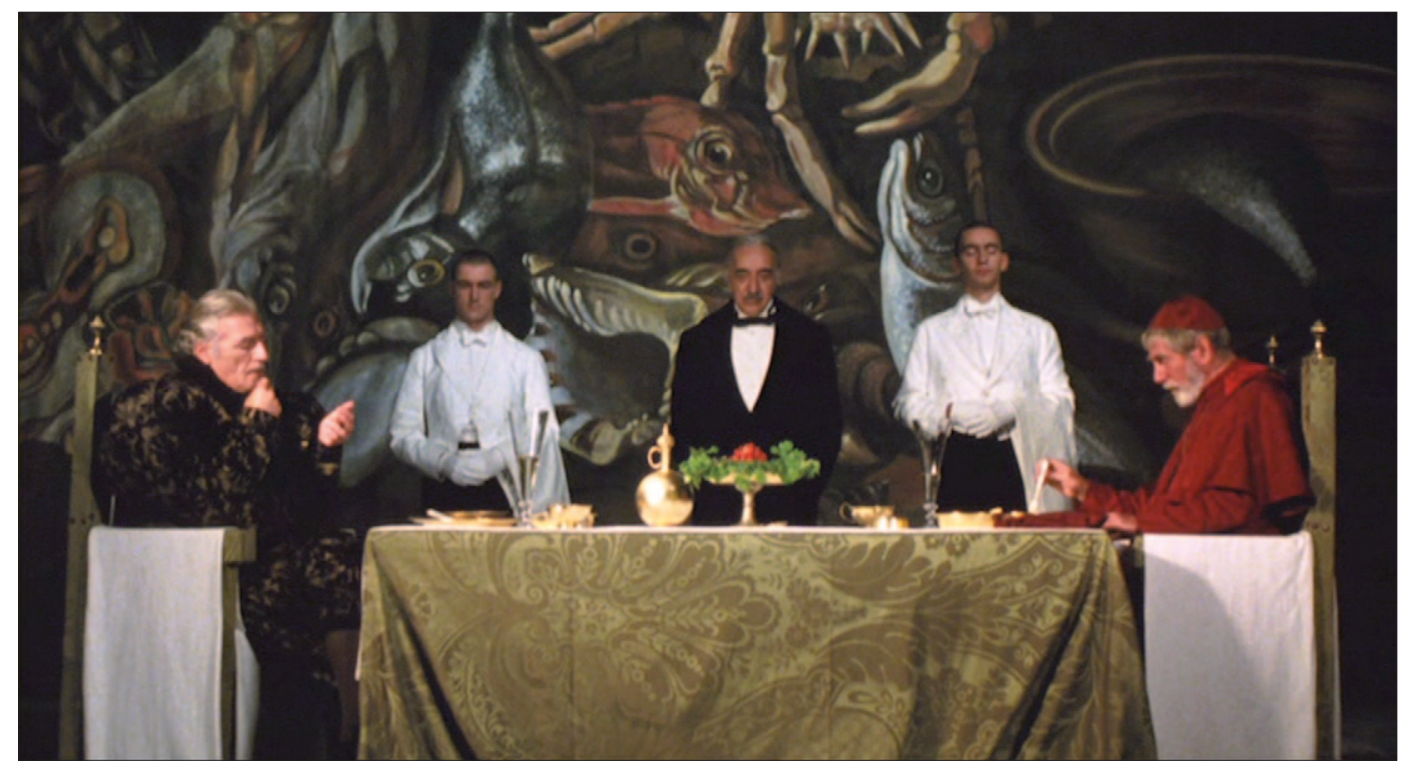

Abb. 4.12 - Giustiniani und Kardinal del Monte beim Essen. Einst aus CARAVAGgio, Sequ. 27

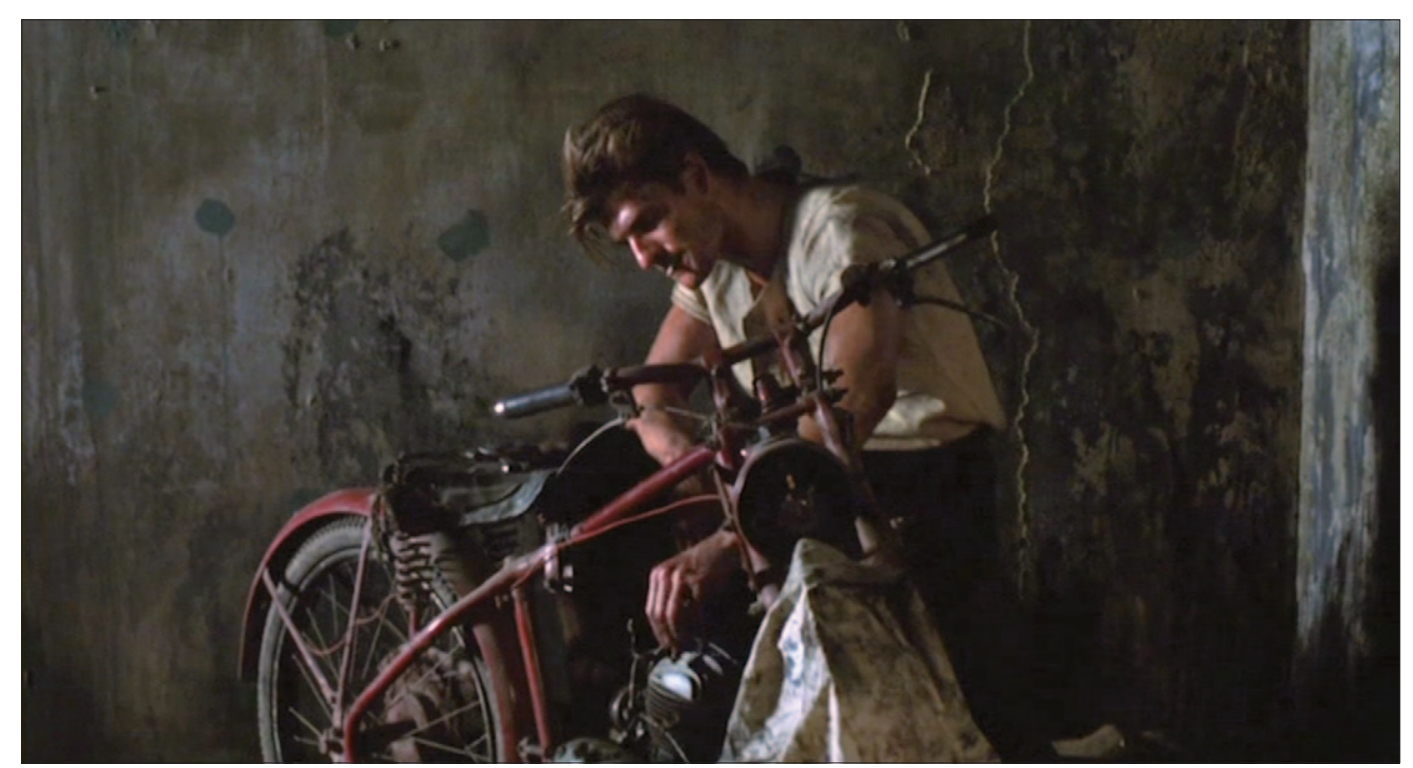

Abb. 4.13 - Ranuccio repariert ein Motorrad. Einst. aus CARAVAGgio, Sequ. 29

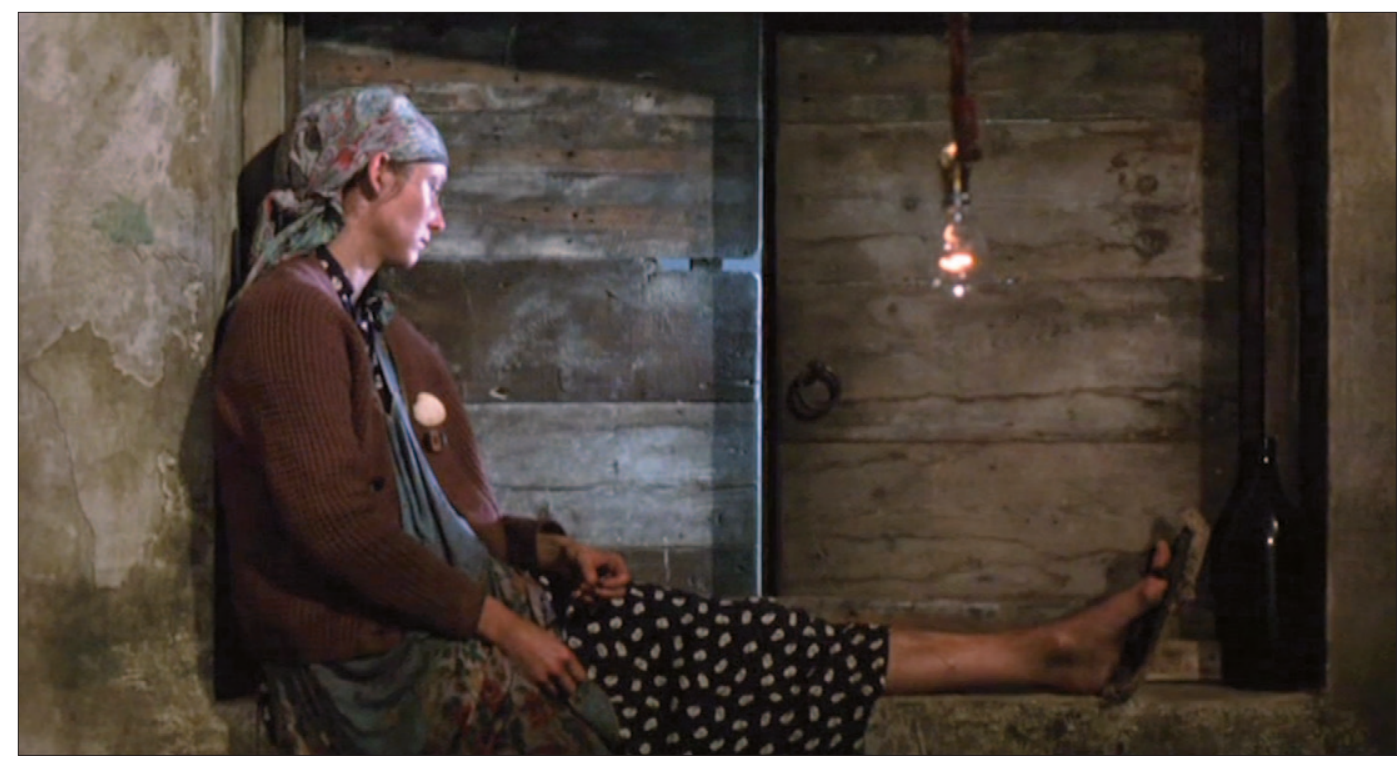

Abb. 4.14 - Lena am Fenster. Einst. aus CARAVAggio, Sequ. 29 


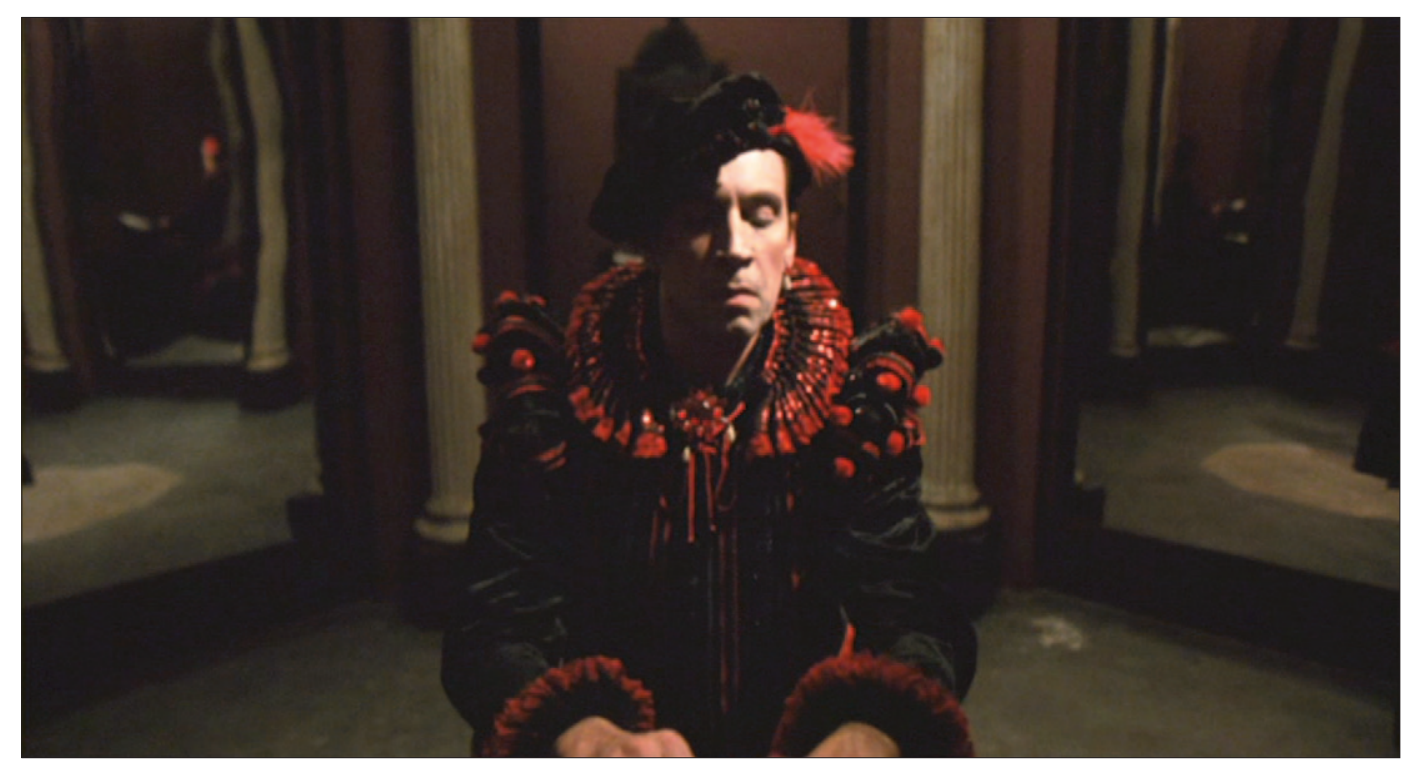

Abb. 4.15 - Baglione an der Schreibmaschine. Einst. aus CARAVAggio, Sequ. 31

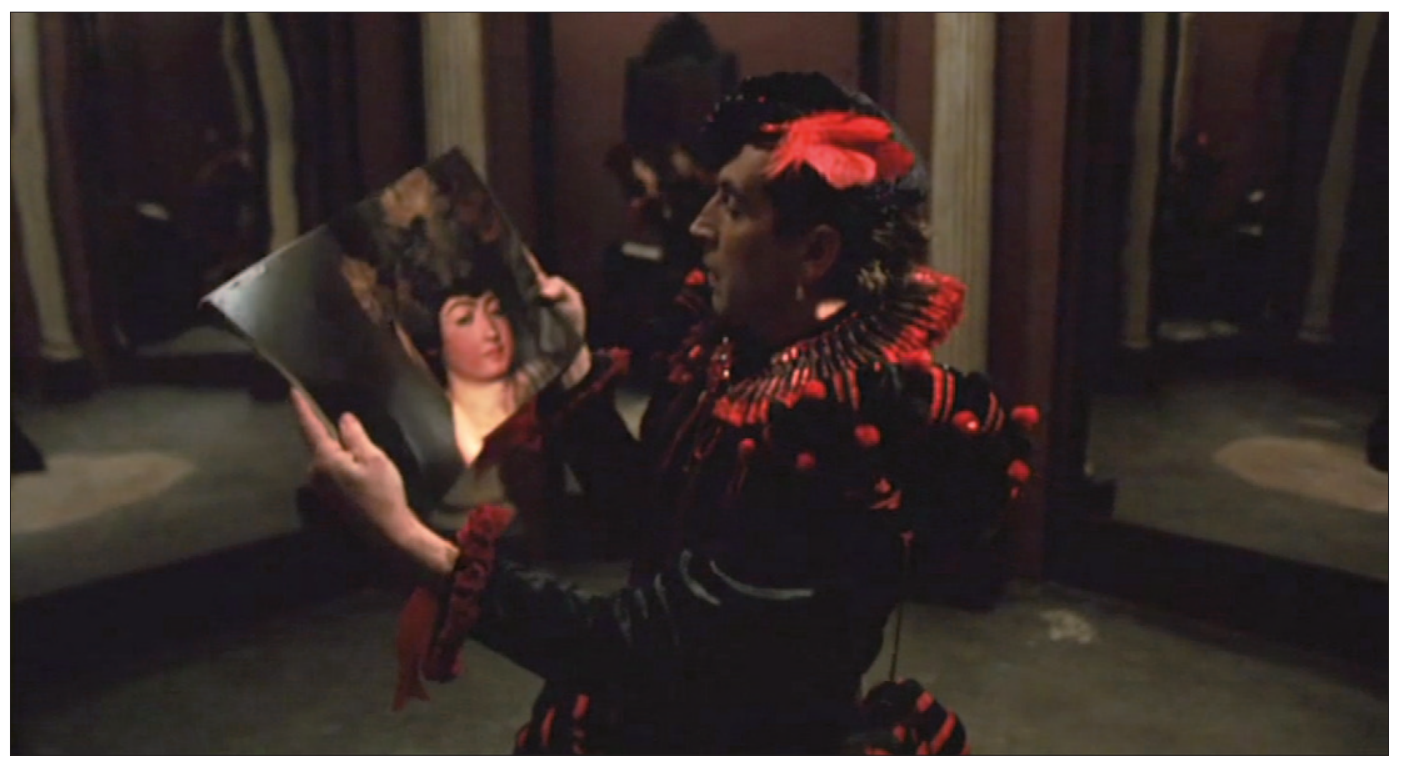

Abb. 4.16 - Baglione mit FMR. Einst. aus CARAVAggio, Sequ. 31 


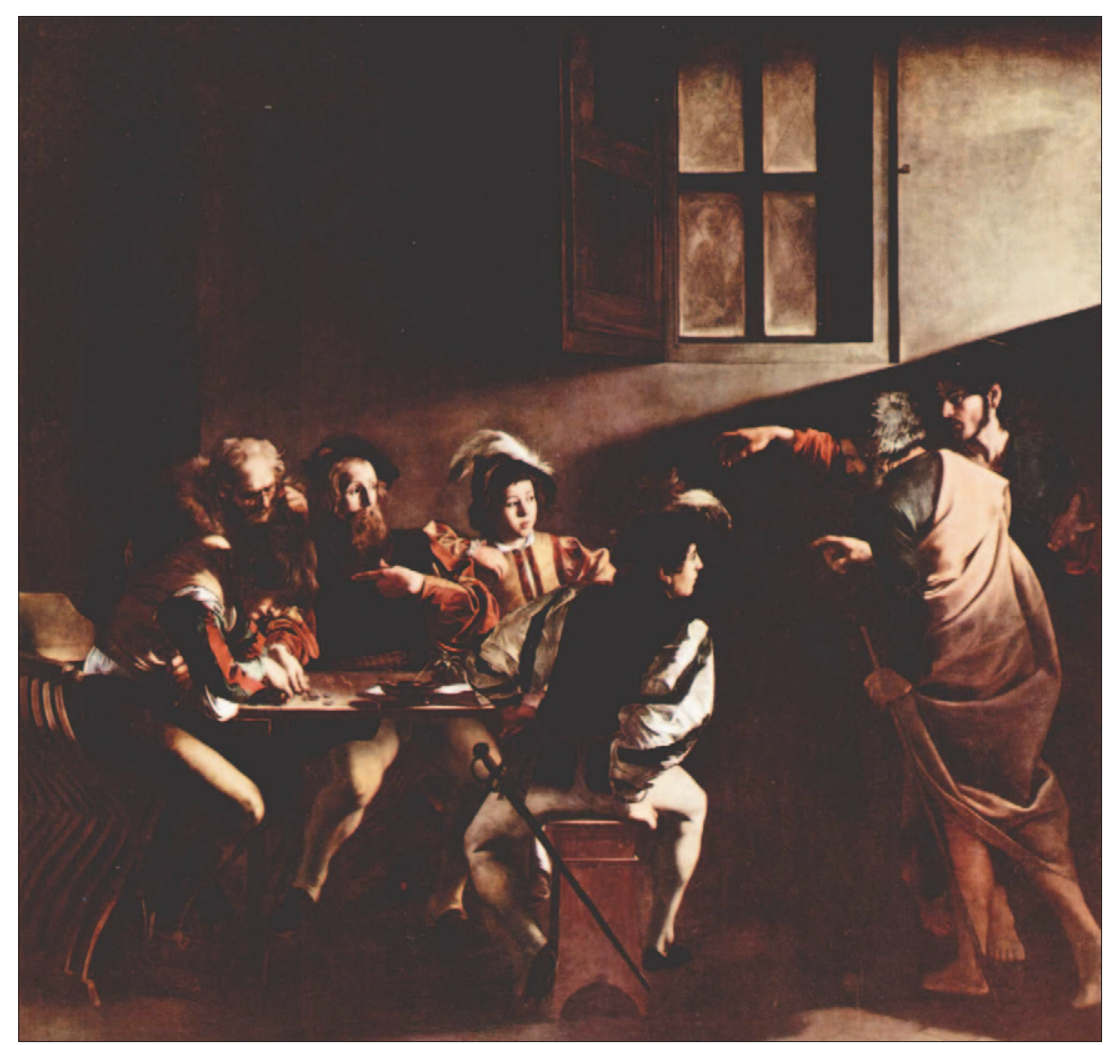

Abb. 4.17- Caravaggio: Berufung des hl. Matthäus. 1600, $322 \mathrm{~cm} \times$ $340 \mathrm{~cm}$, San Luigi dei Francesi, Cappella Contarelli, Rom.

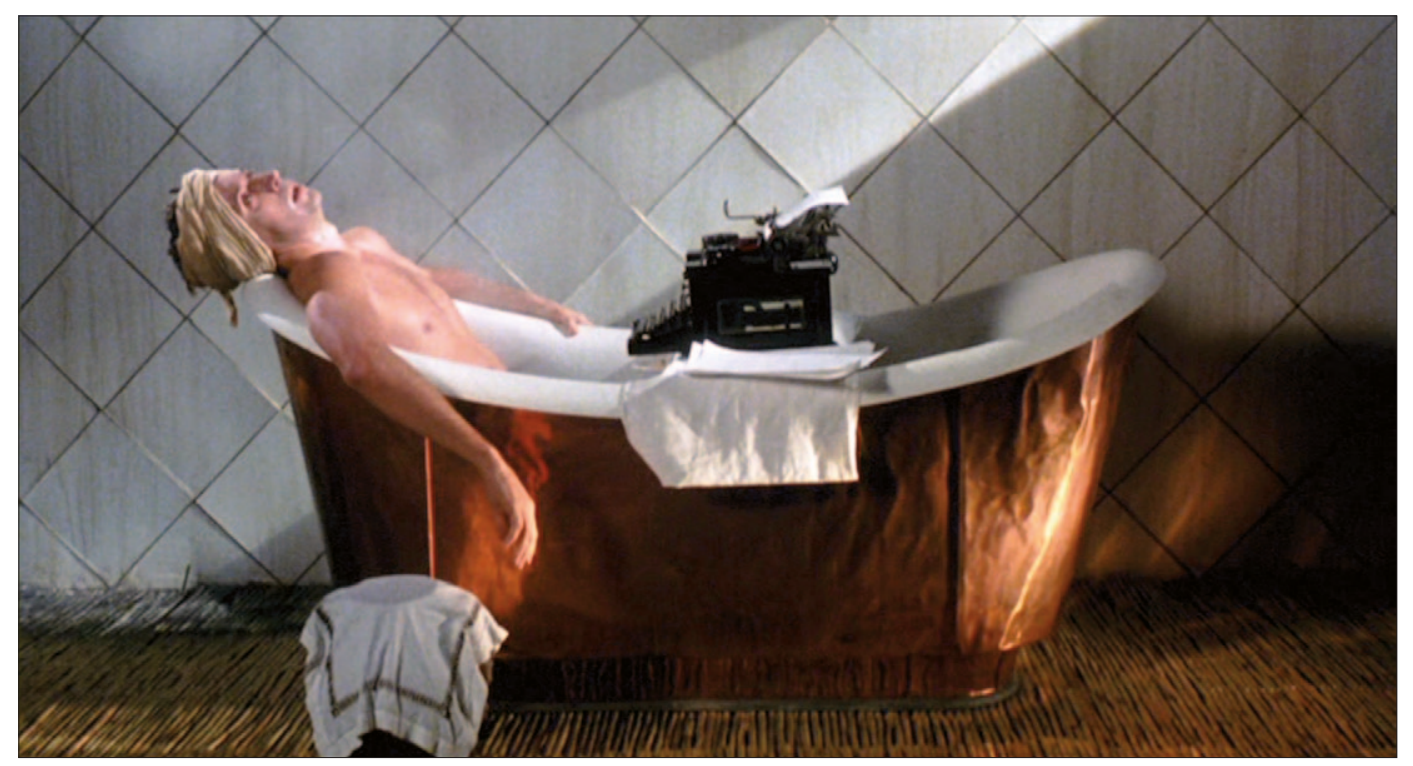

Abb. 4.18- Baglione in der Wanne. Einst. aus CARAVAggio, Sequ. 35 


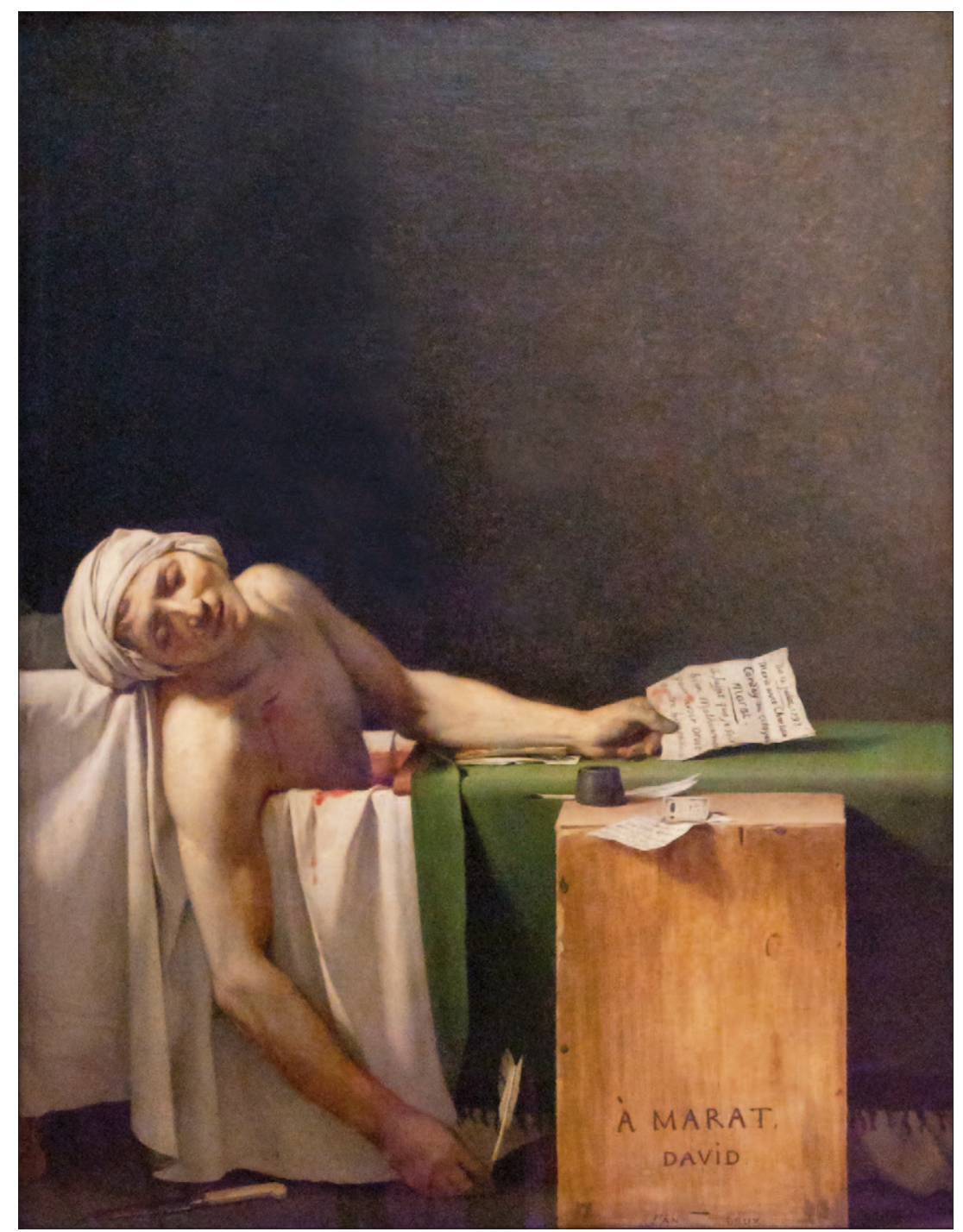

Abb. 4.19- Jacques-Louis David: Der Tod des Marat. 1793, Öl auf Leinwand, $162 \mathrm{~cm} \times 128 \mathrm{~cm}$, Königliche Museen der Schönen Künste, Brüssel.

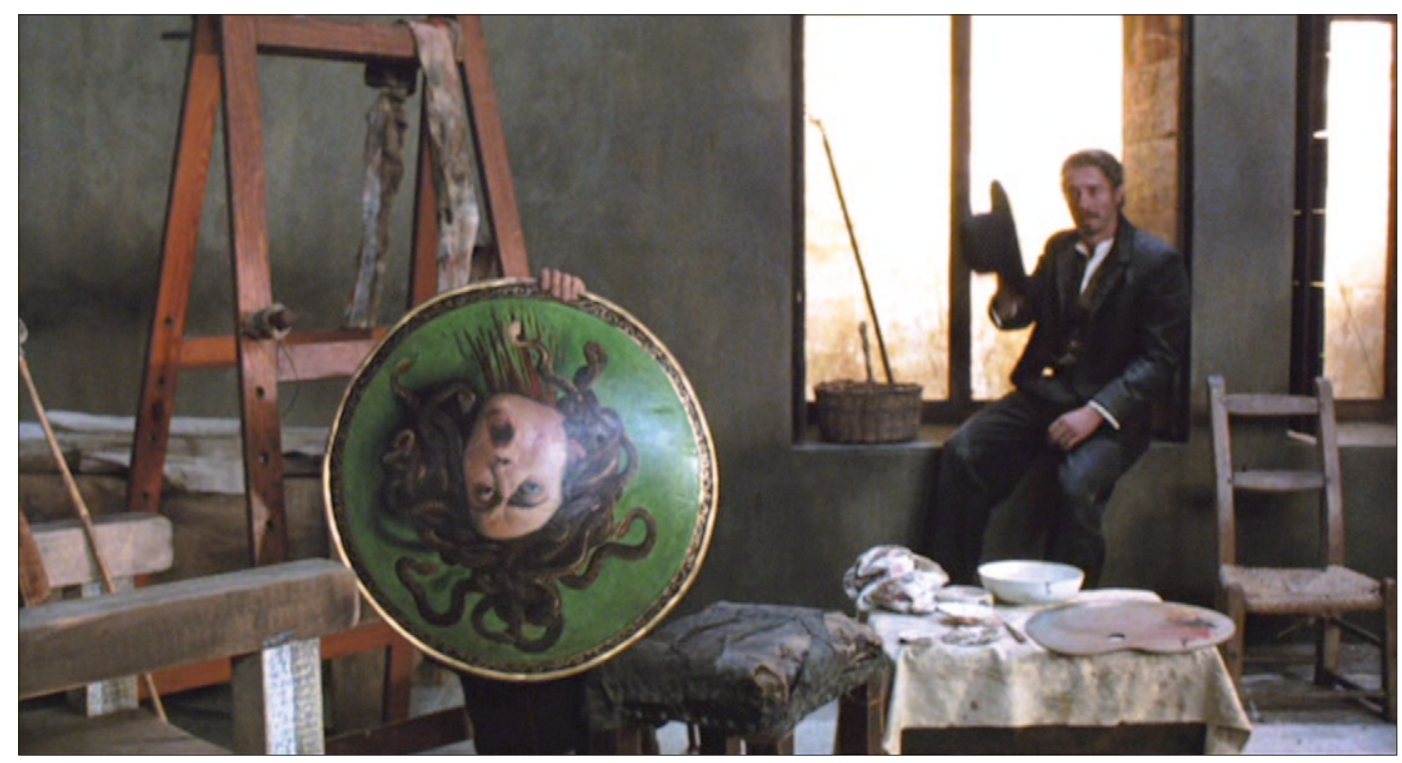

Abb. 4.20- Jerusaleme mit dem Medusenschild. Einst. aus CARAVAGgio, Sequ. 3 


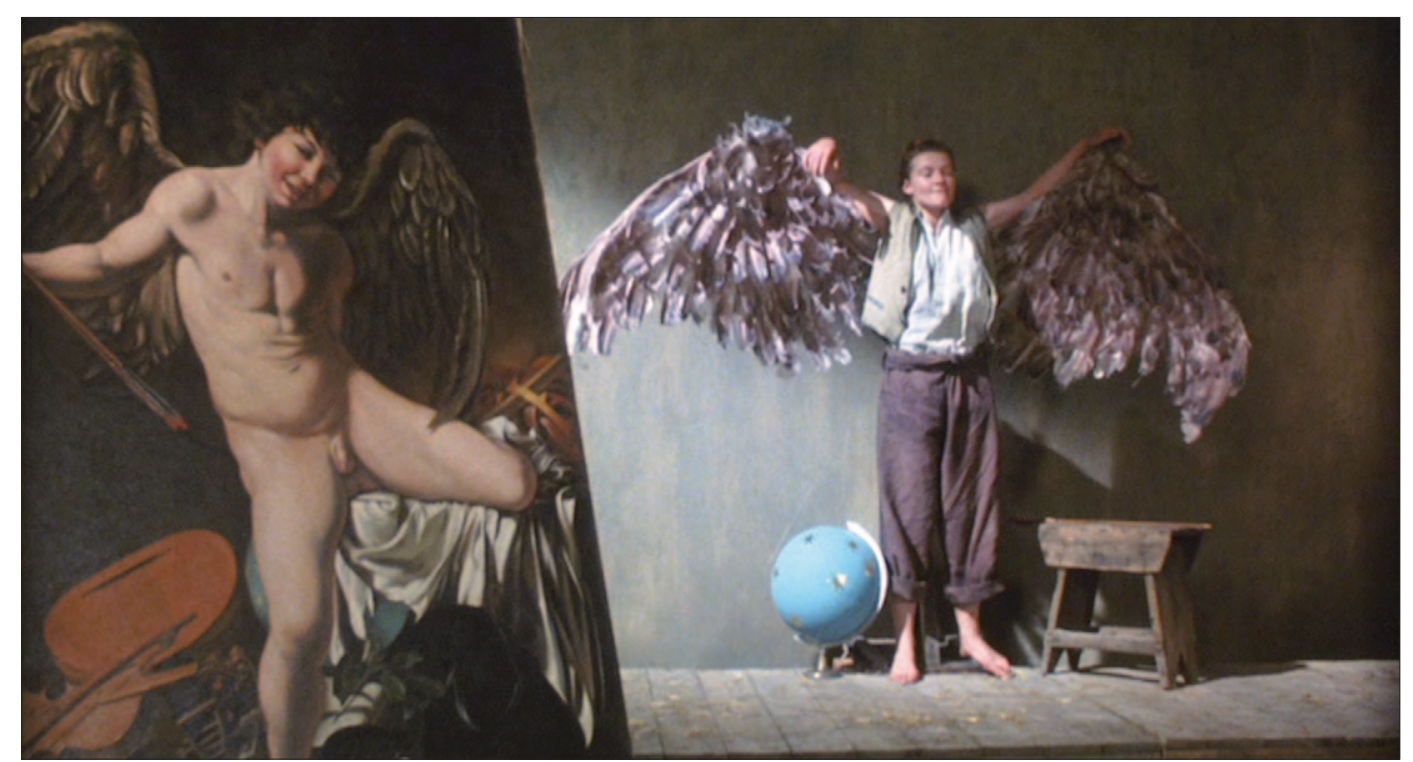

Abb. 4.21- Amor als Sieger im Film. Einst. aus CARAVAGgio, Sequ. 28

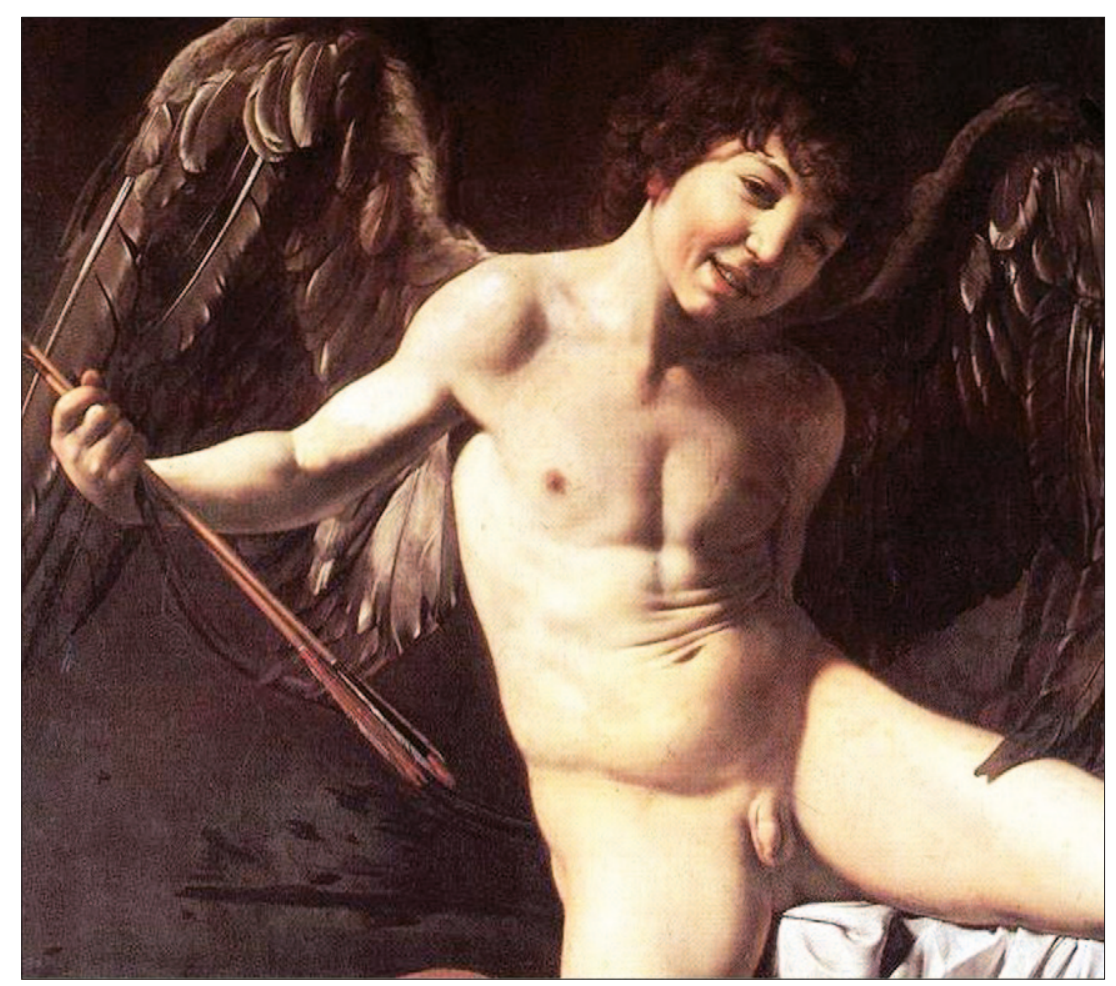

Abb. 4.22- Detail aus Caravaggio: Amor als Sieger. 1601/o2, $156 \mathrm{~cm} \times 113 \mathrm{~cm}$, Staatliche Museen, Gemäldegalerie, Berlin. 


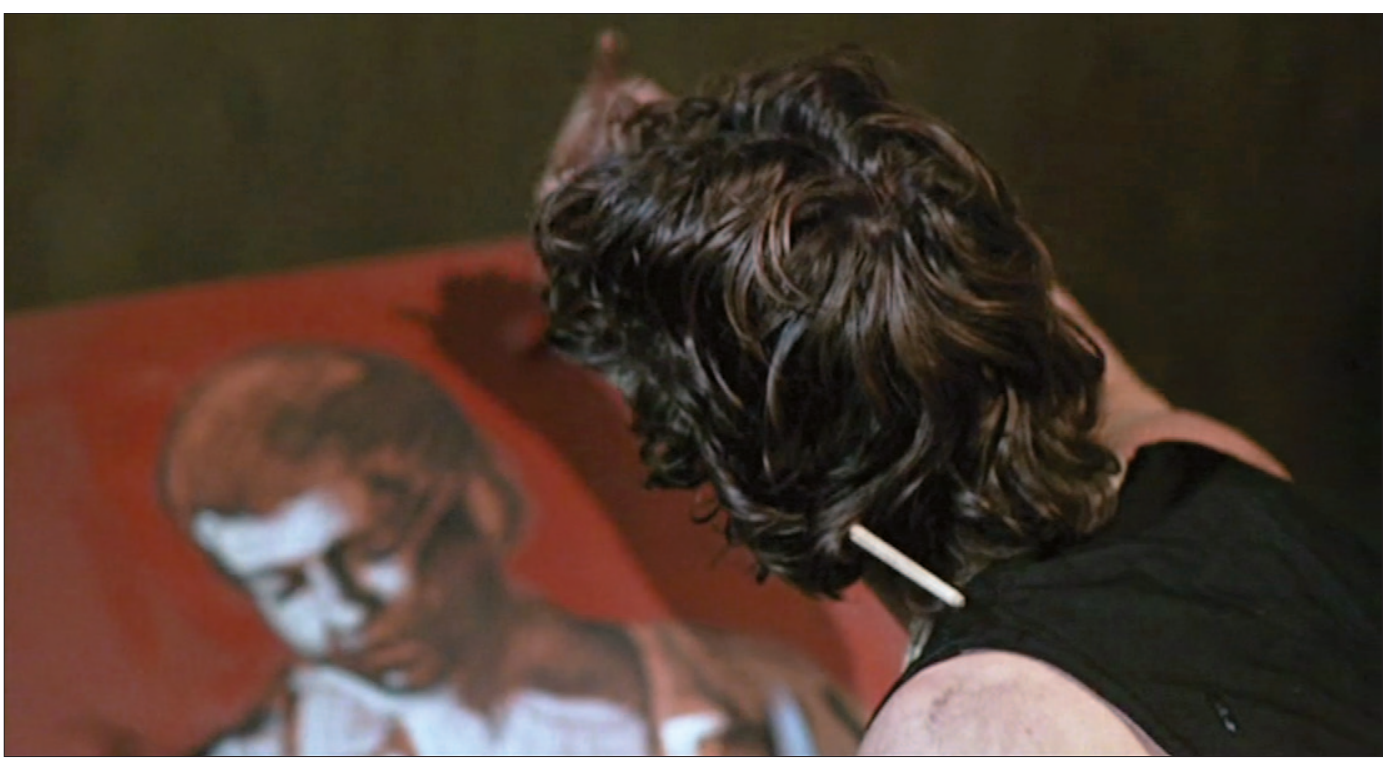

Abb. 4.23- Michele malt den Fruchtschälenden Knaben. Einst. aus CARAVAGgio, Sequ. 5

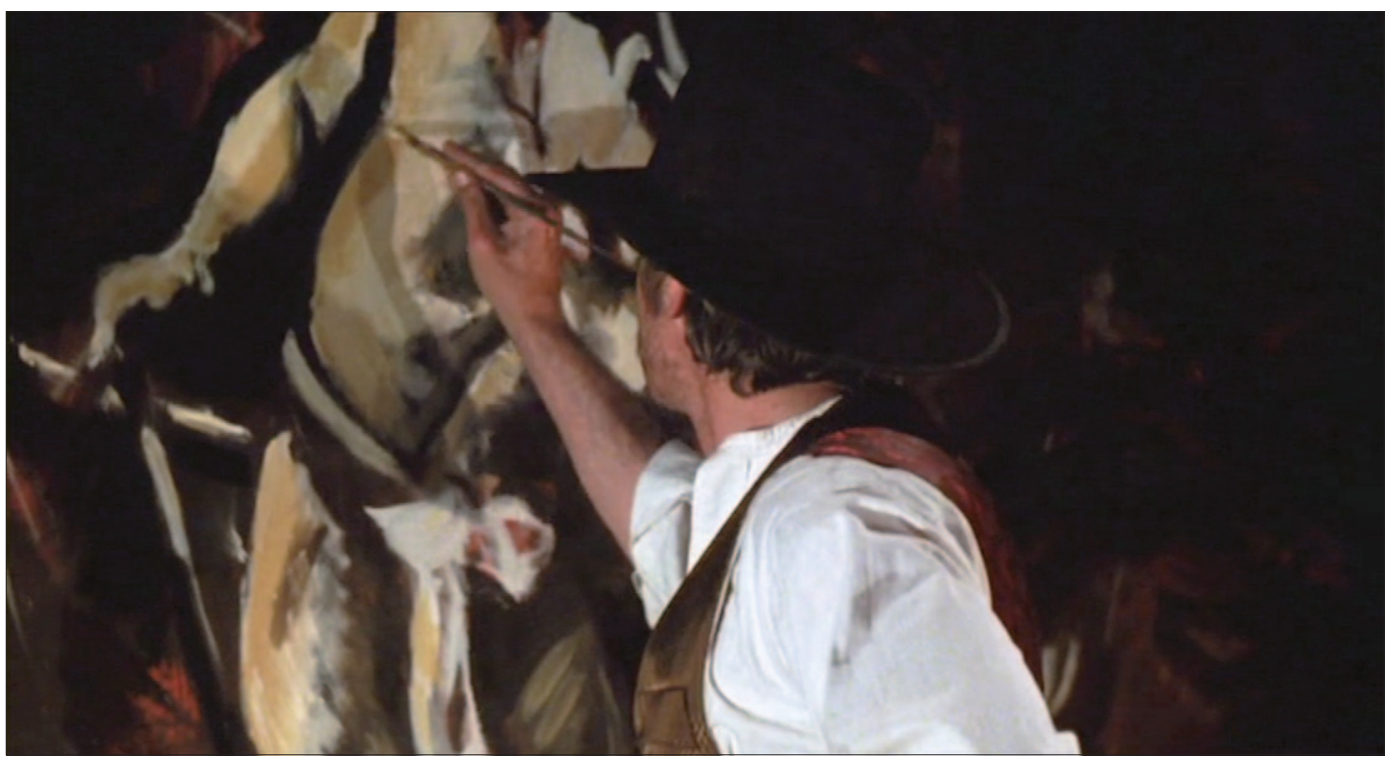

Abb. 4.24- Michele malt die Ermordung des hl. Matthäus. Einst. aus CARAVAGgio, Sequ. 17

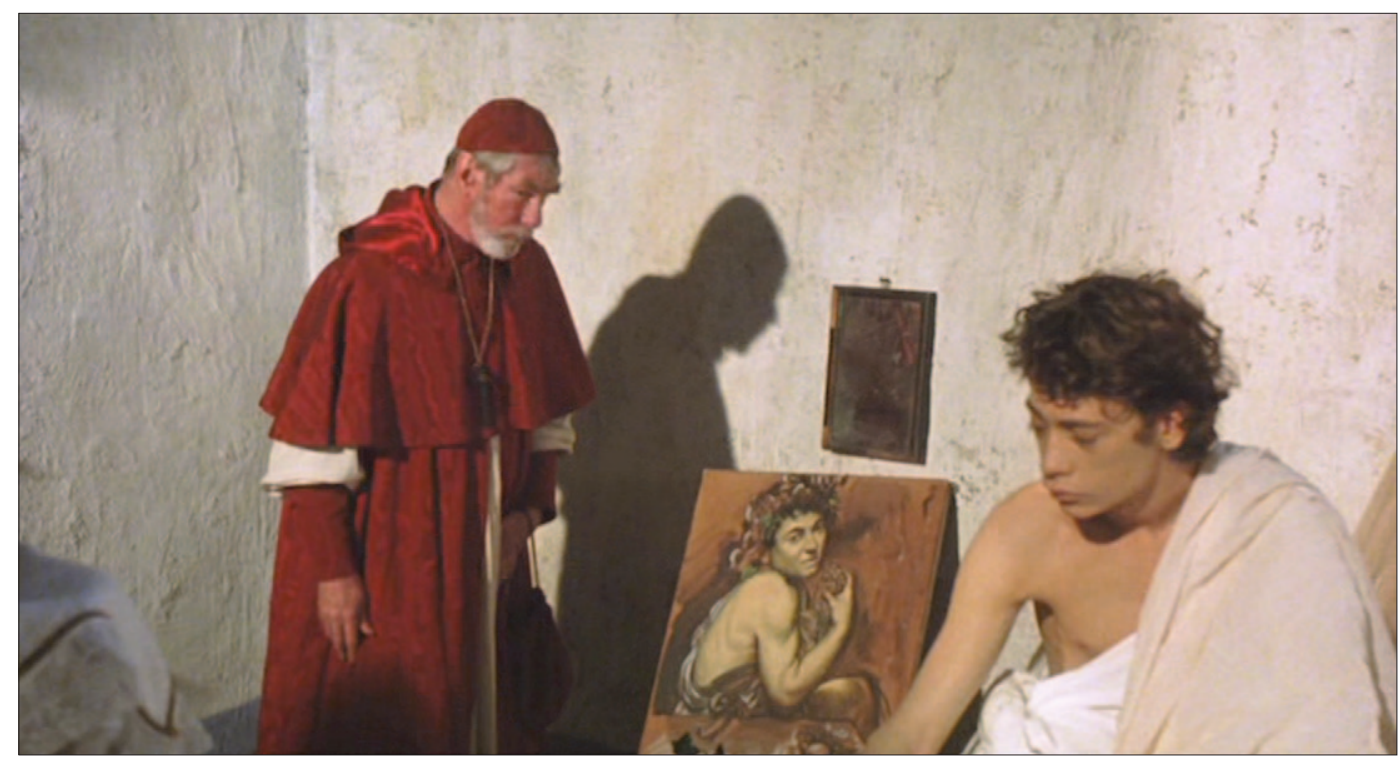

Abb. 4.25- Kardinal del Monte und Michele am halb fertigen Bacchino malato. Einst. aus CARAVAgGio, Sequ. 7 


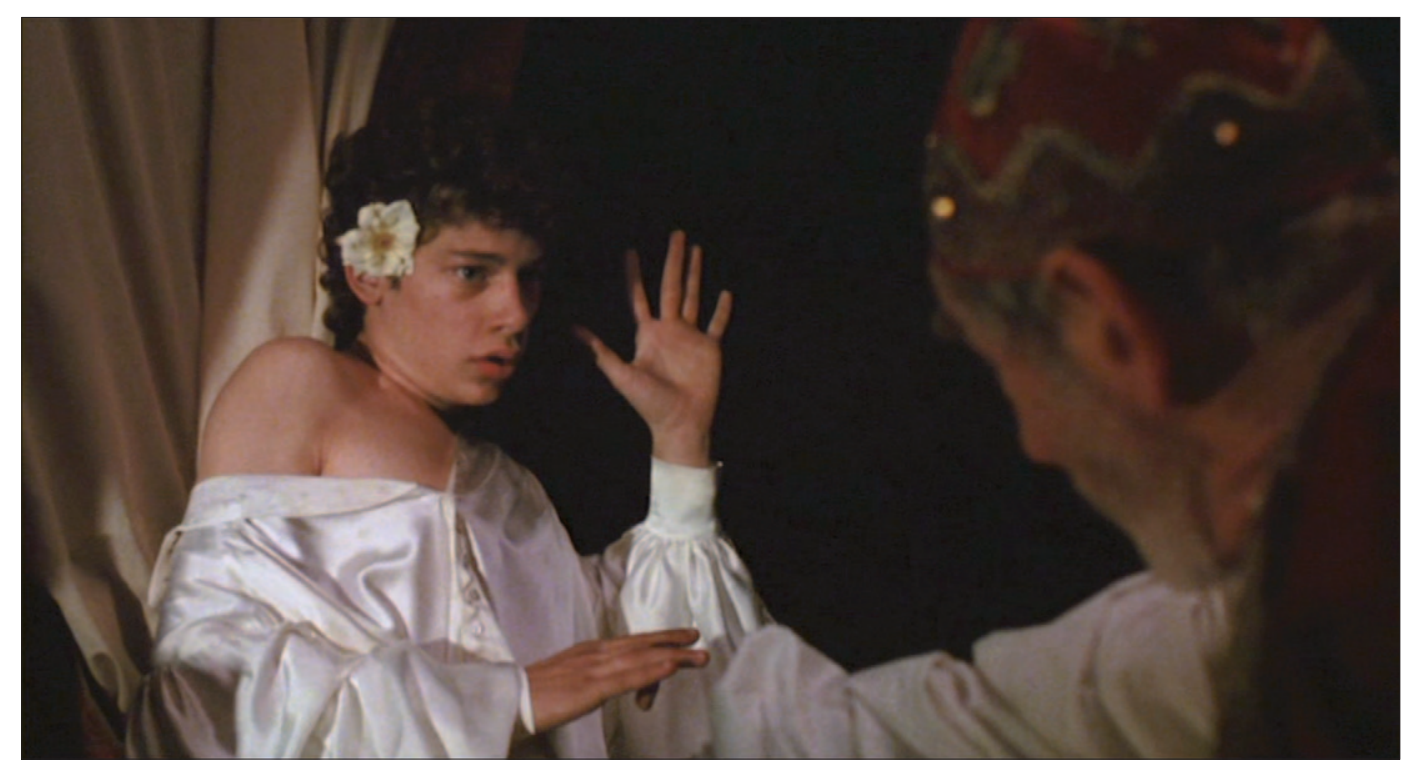

Abb. 4.26- Michele wie der Jüngling, von einer Eidechse gebissen. Einst. aus CARAVAGGIO, Sequ. 9

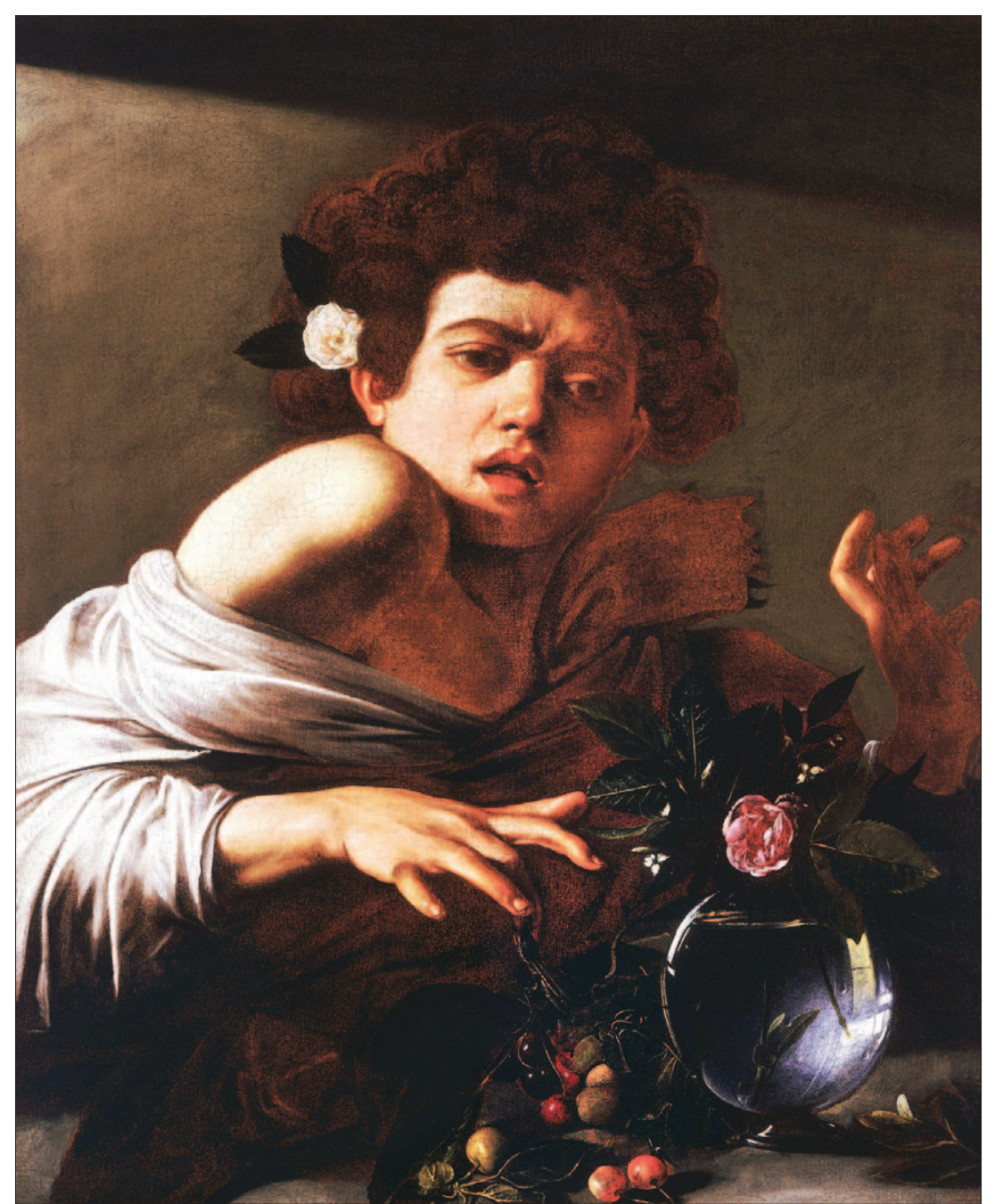

Abb. 4.27-Caravaggio: Jüngling, von einer Eidechse gebissen. $66 \mathrm{~cm} \times$ 49,5 cm, National Gallery, London. 


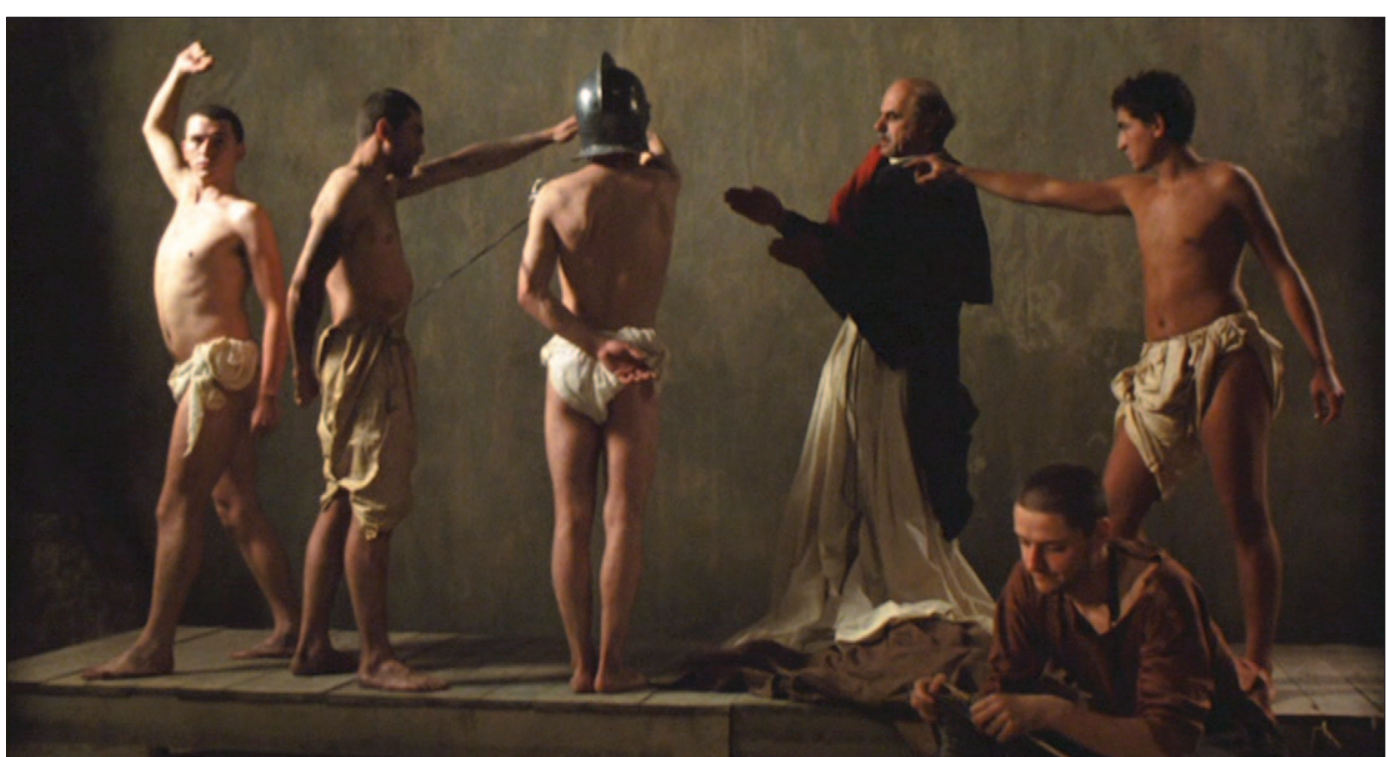

Abb. 4.28- Modelle für die 1. Fassung des Martyrium des hl. Matthäus. Einst. aus CARAVAGGIO, Sequ. 13

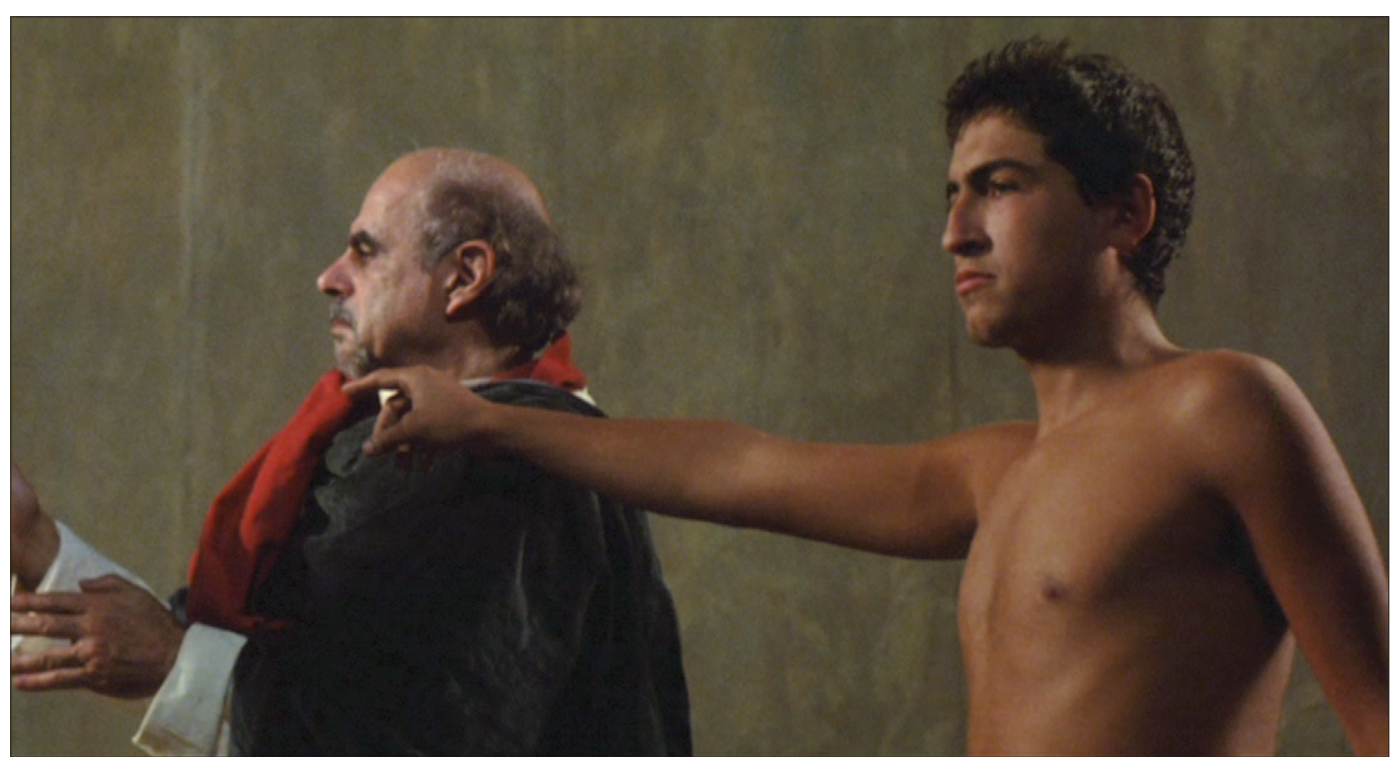

Abb. 4.29- Modelle für die 1. Fassung des Martyrium des hl. Matthäus. Einst. aus CARAVAGGIO, Sequ. 13

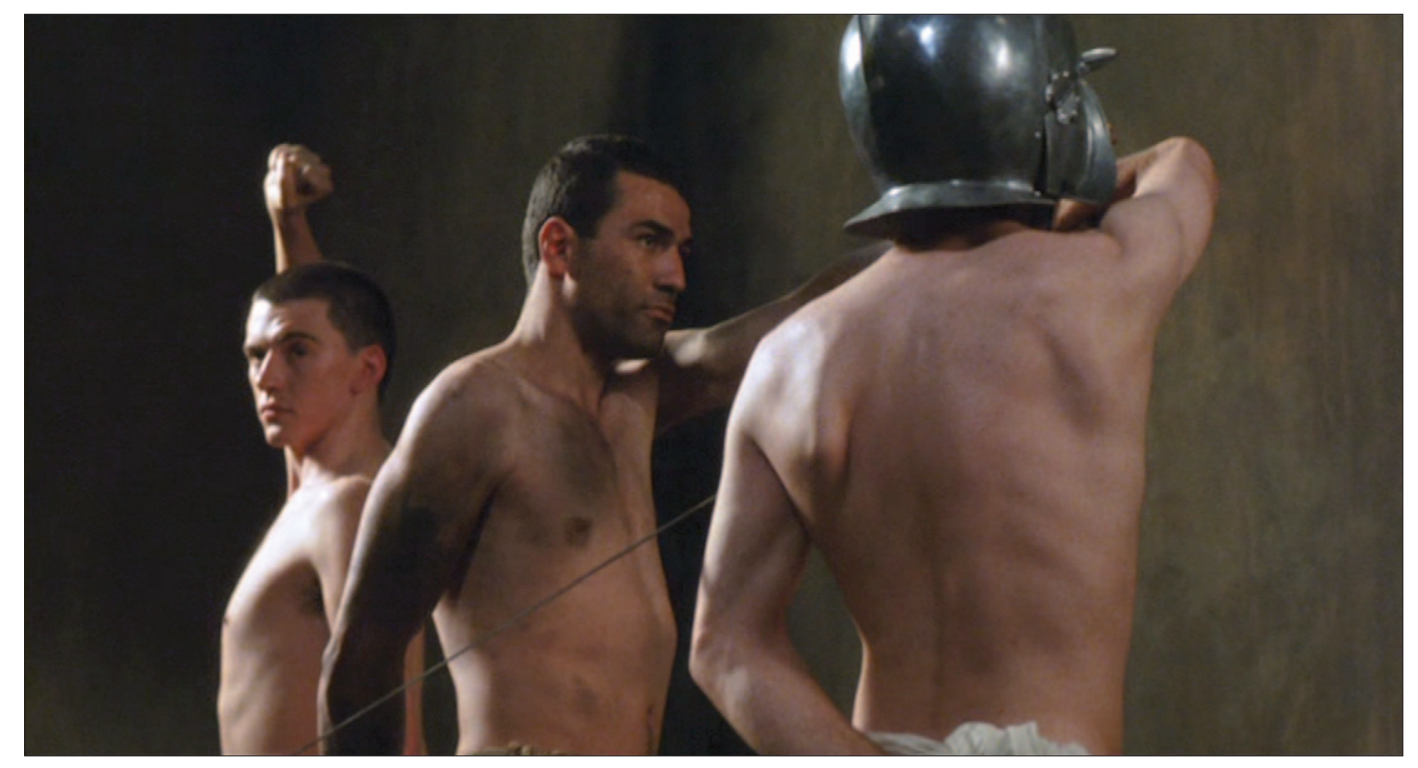

Abb. 4.30- Modelle für die 1. Fassung des Martyrium des hl. Matthäus. Einst. aus CARAVAGGIO, Sequ. 13 


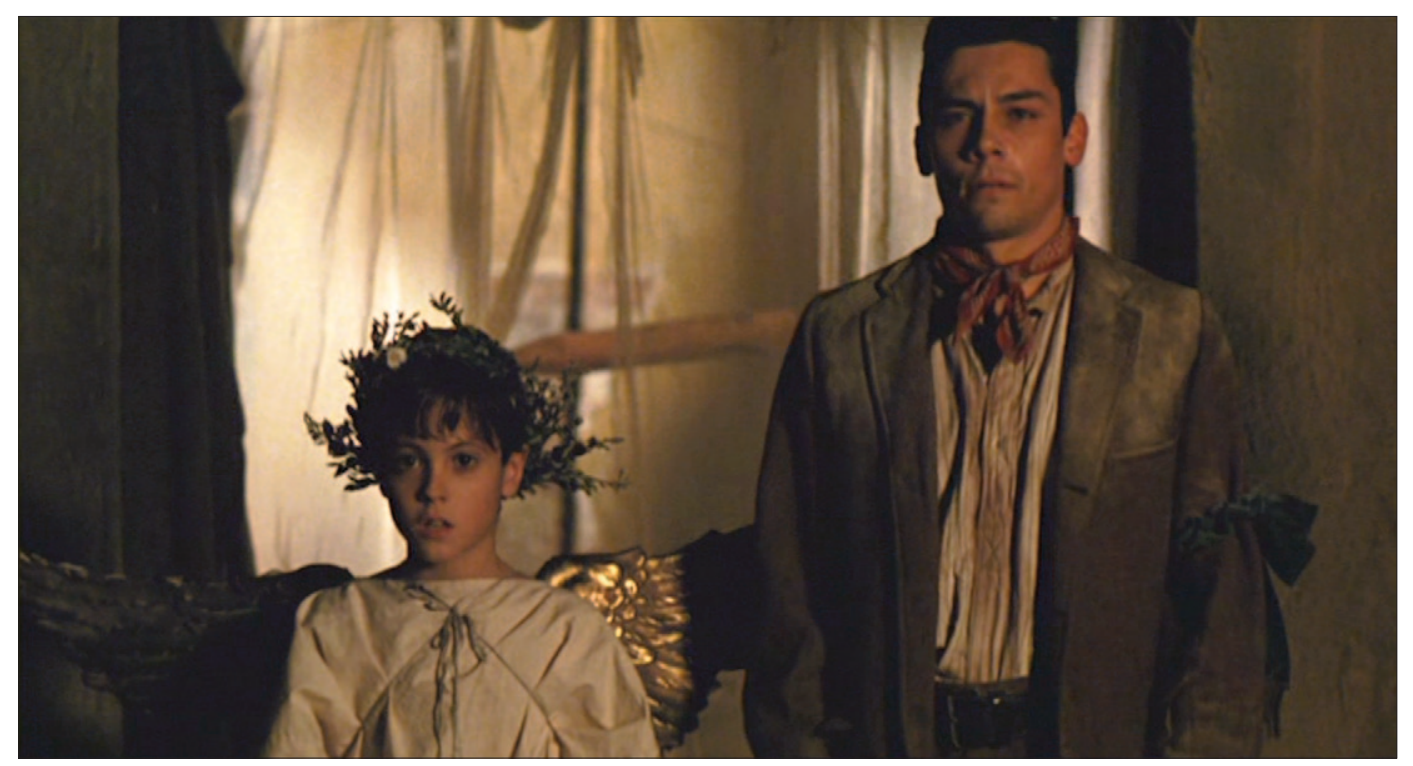

Abb. 4.31- Michele und Pasqualone. Einst. aus CARAVAGgio, Sequ. 57

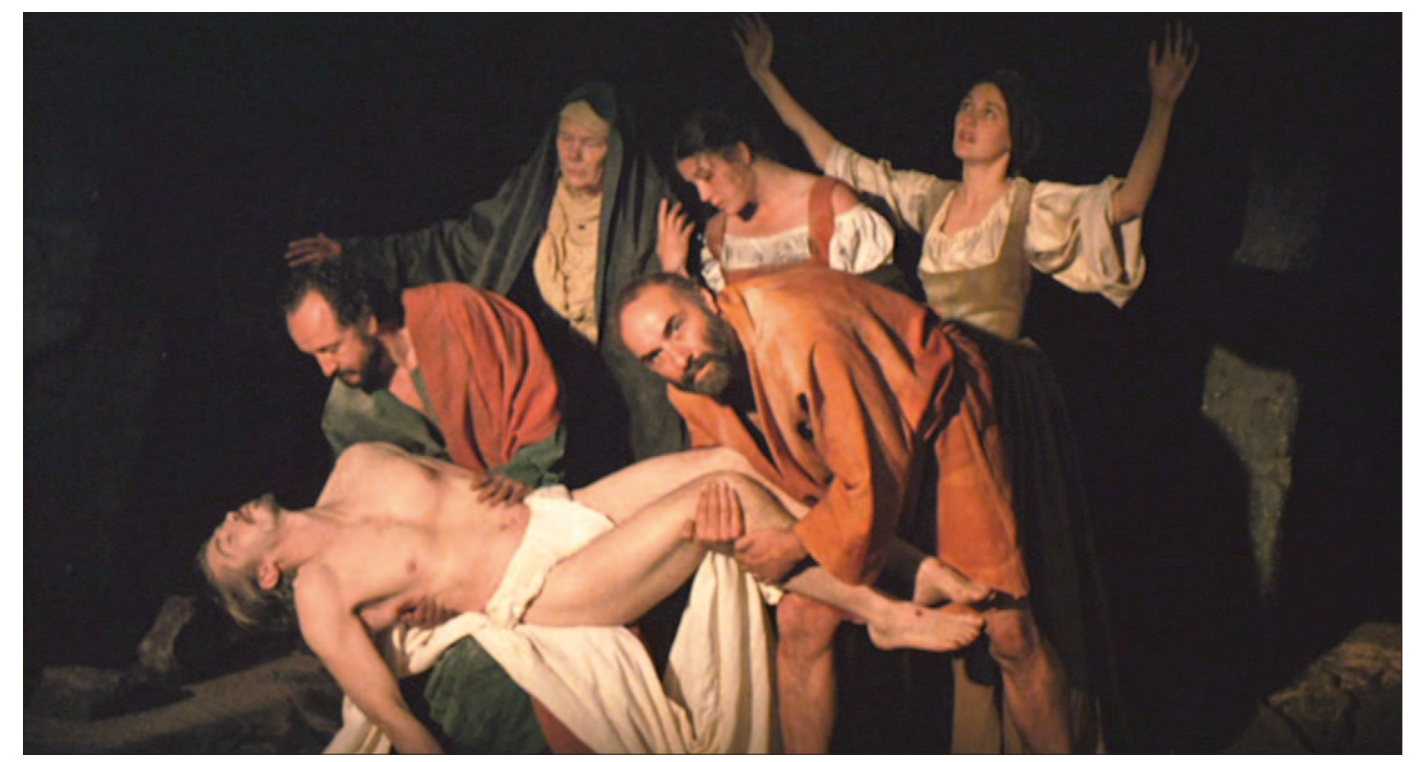

Abb. 4.32- Tableau vivant der Grablegung Christi. Einst. aus CARAvaggio, Sequ. 57 

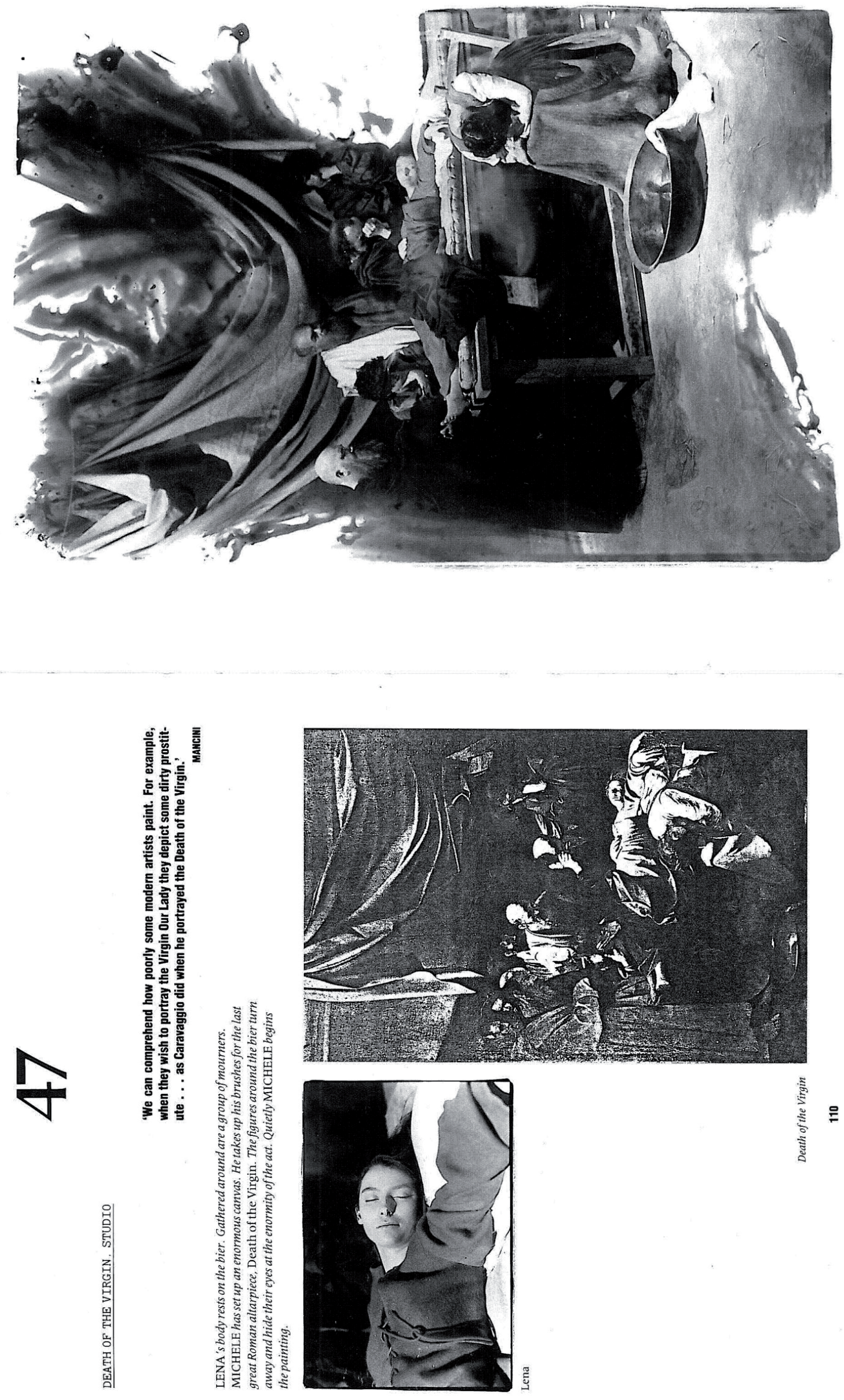

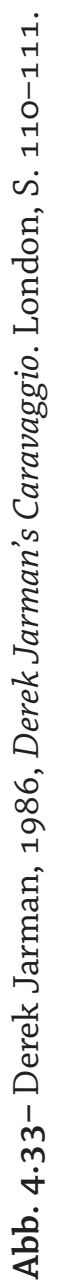




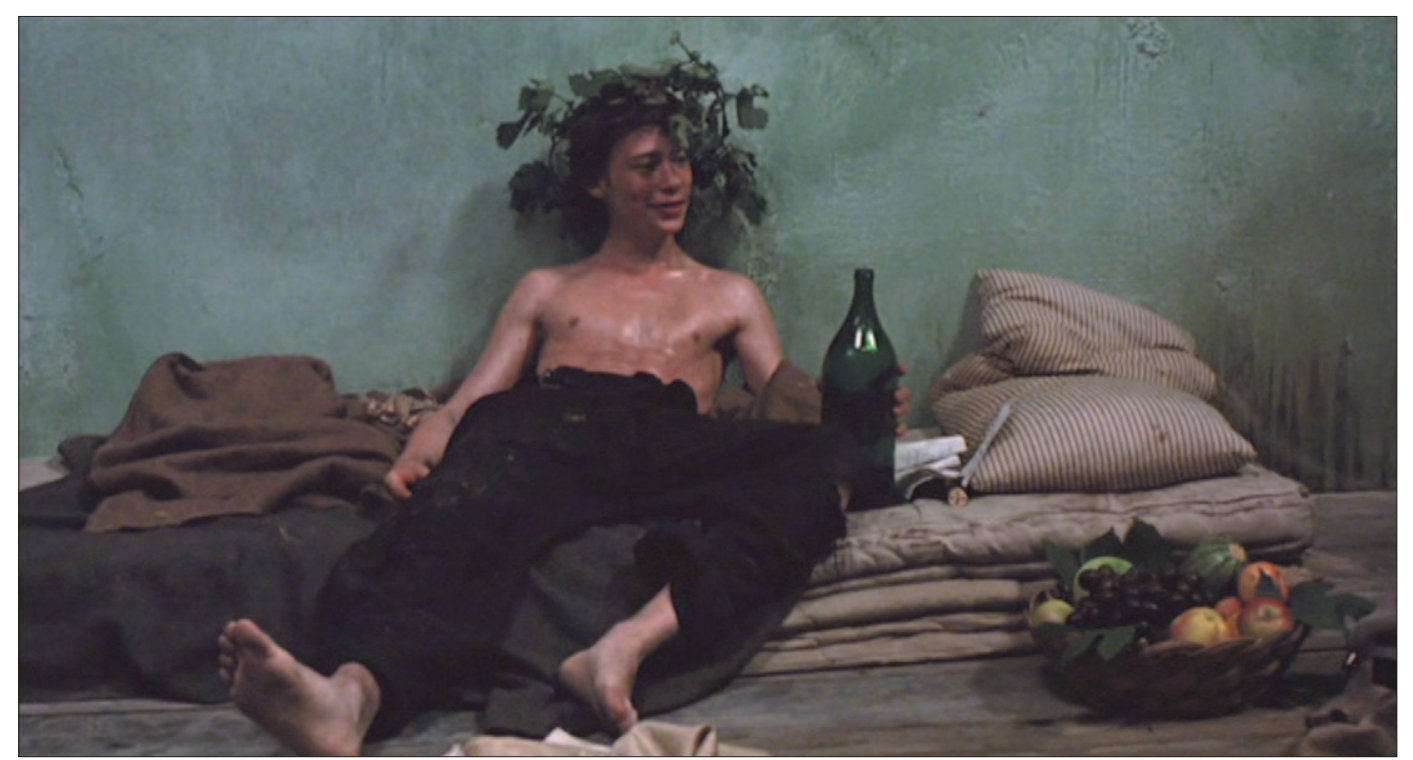

Abb. 4.34- Michele als Bacchus. Einst. aus CARAvaggio, Sequ. 6 


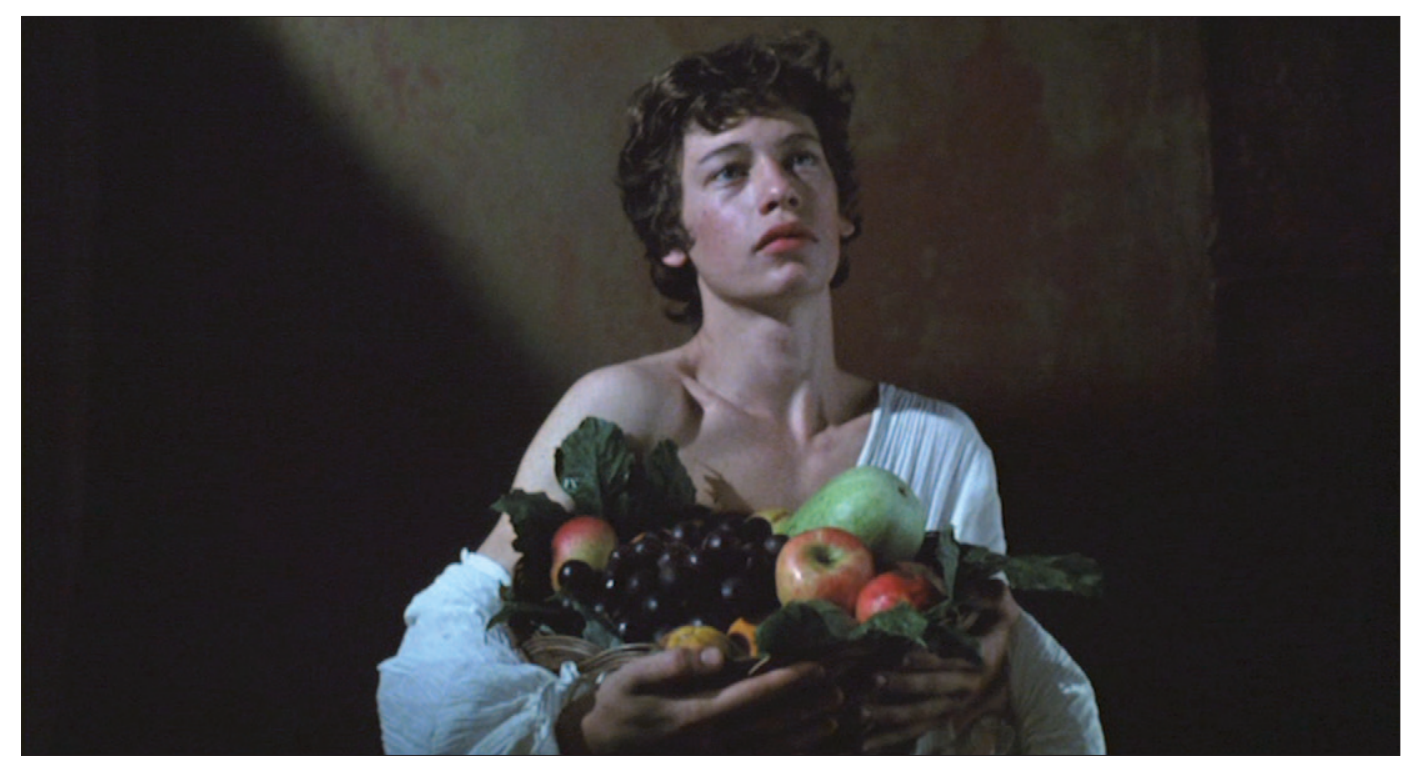

Abb. 4.35- Michele als Knabe mit dem Früchtekorb. Einst. aus CARAVAggio, Sequ. 6

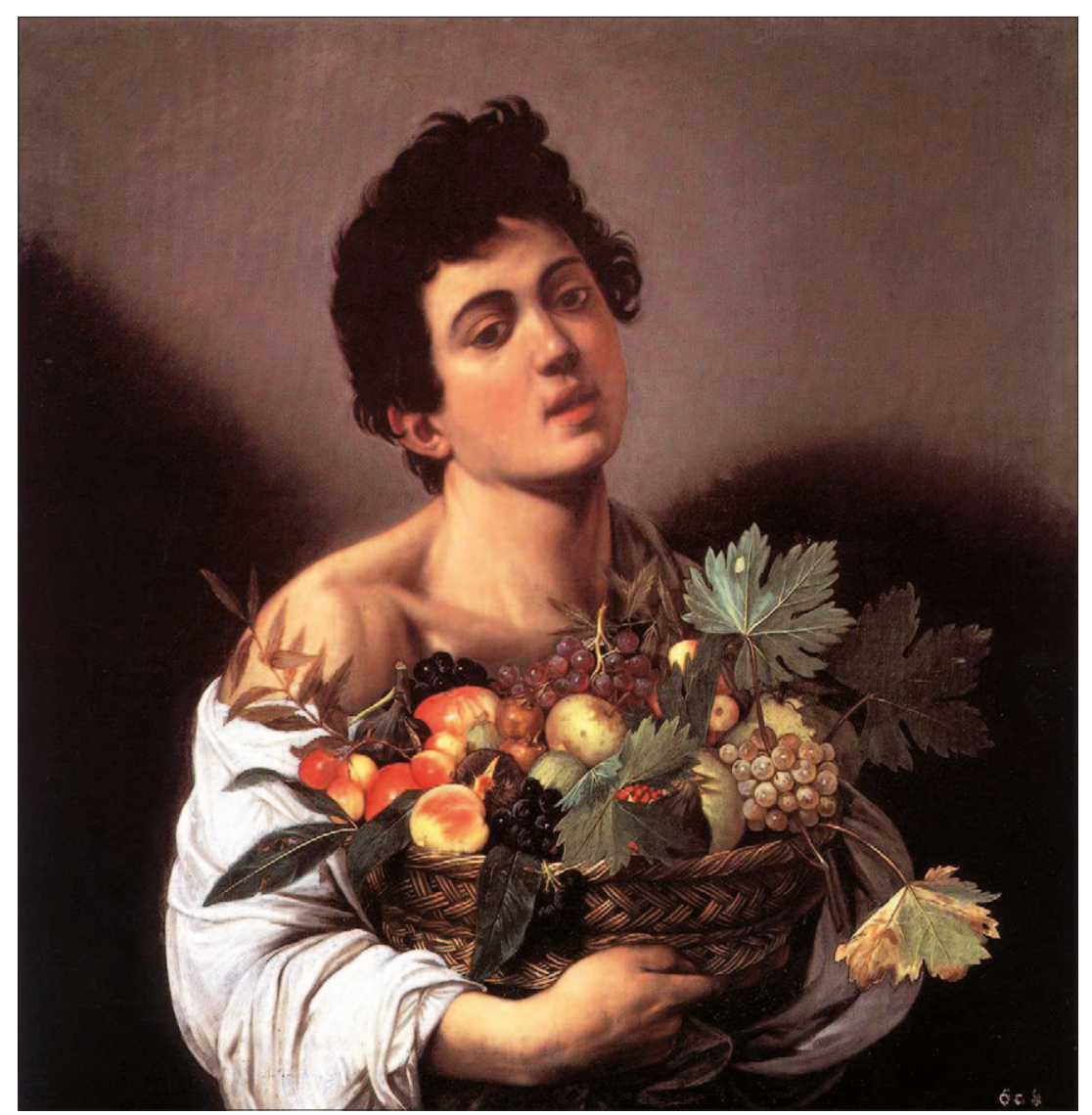

Abb. 4.36- Caravaggio: Knabe mit Früchtekorb. 1593/94, $70 \mathrm{~cm} \times 67$ $\mathrm{cm}$, Museo e Galleria Borghese, Rom. 


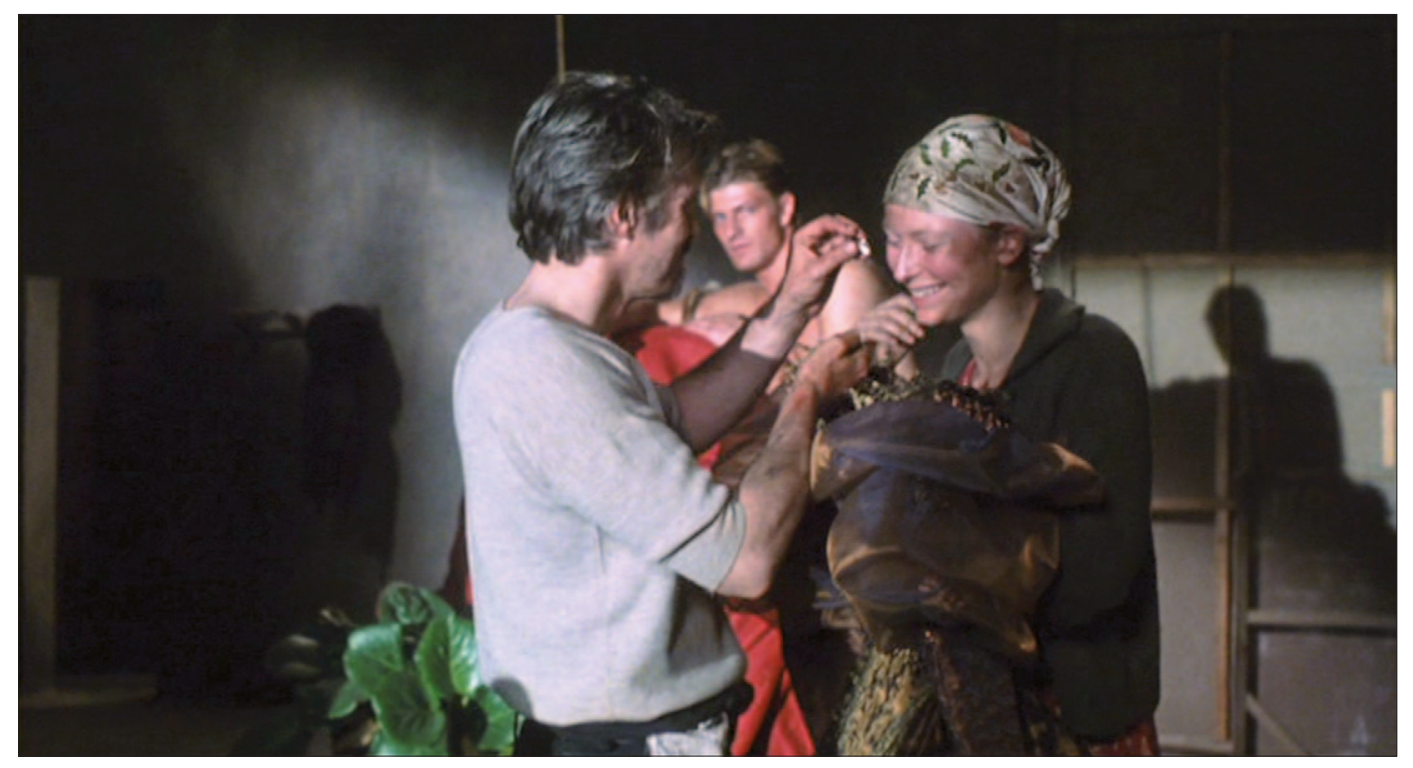

Abb. 4.37- Michele, Lena und Ranuccio. Einst. aus CARAVAGgio, Sequ. 30

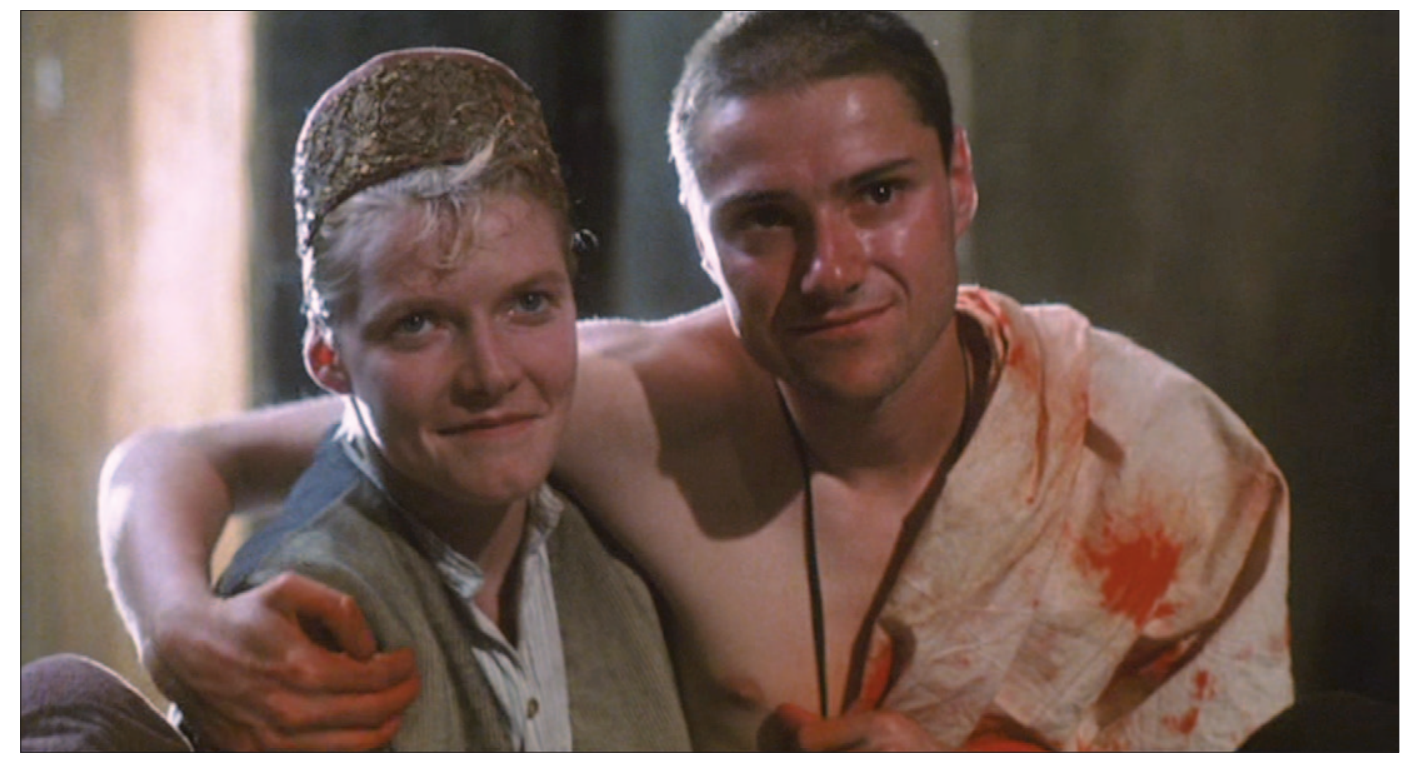

Abb. 4.38- Pipo und Jerusaleme. Einst. aus CARAVAggio, Sequ. 30 
